\title{
Einführung in die Schweizer Agrarpolitik
}

Monograph

Author(s):

Huber, Robert (1)

Publication date:

2022

Permanent link:

https://doi.org/10.3929/ethz-b-000530354

Rights / license:

Creative Commons Attribution-NonCommercial-NoDerivatives 4.0 International

Originally published in:

https://doi.org/10.3218/4059-3 


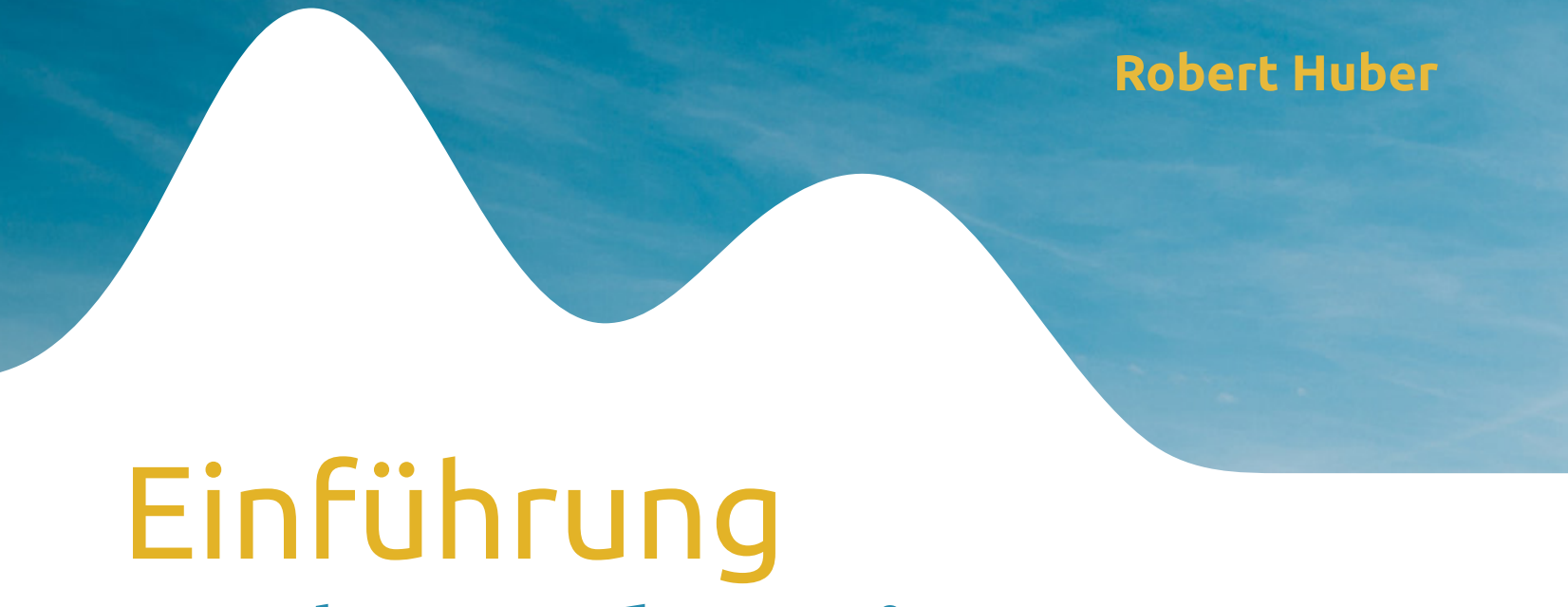

in die Schweizer

Agrarpolitik

14.

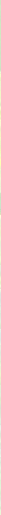




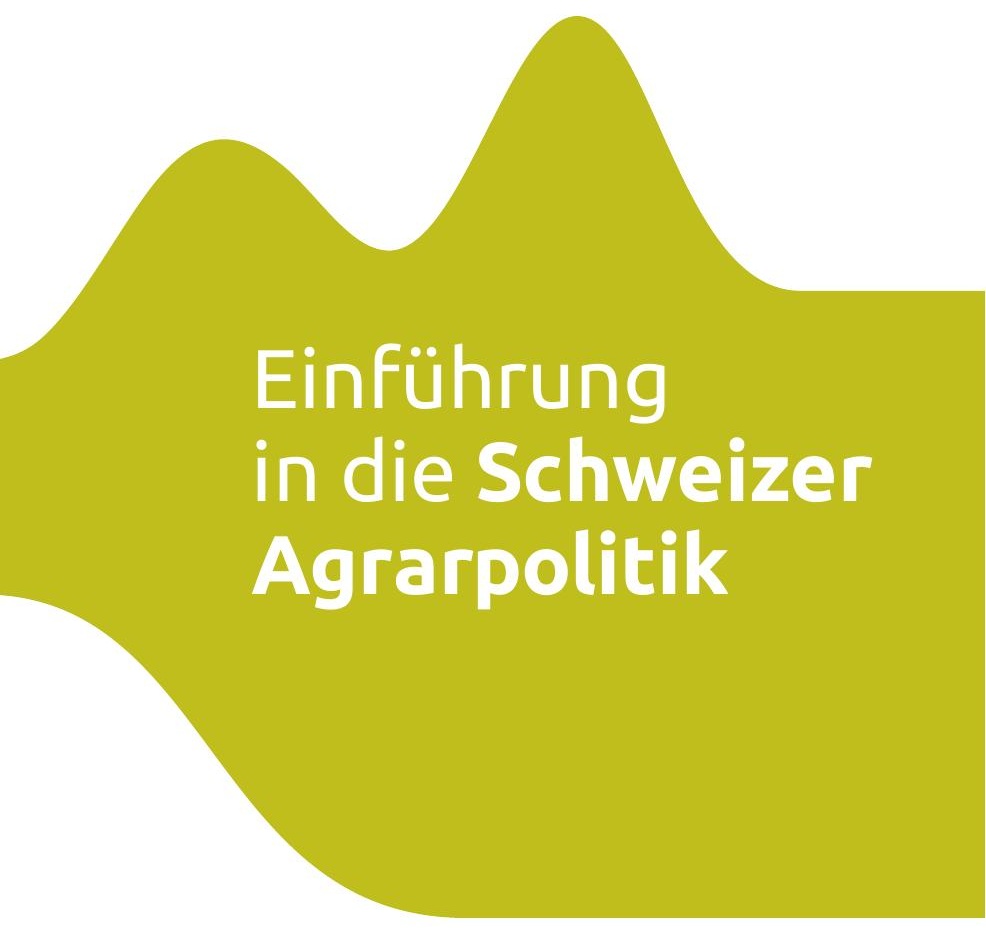



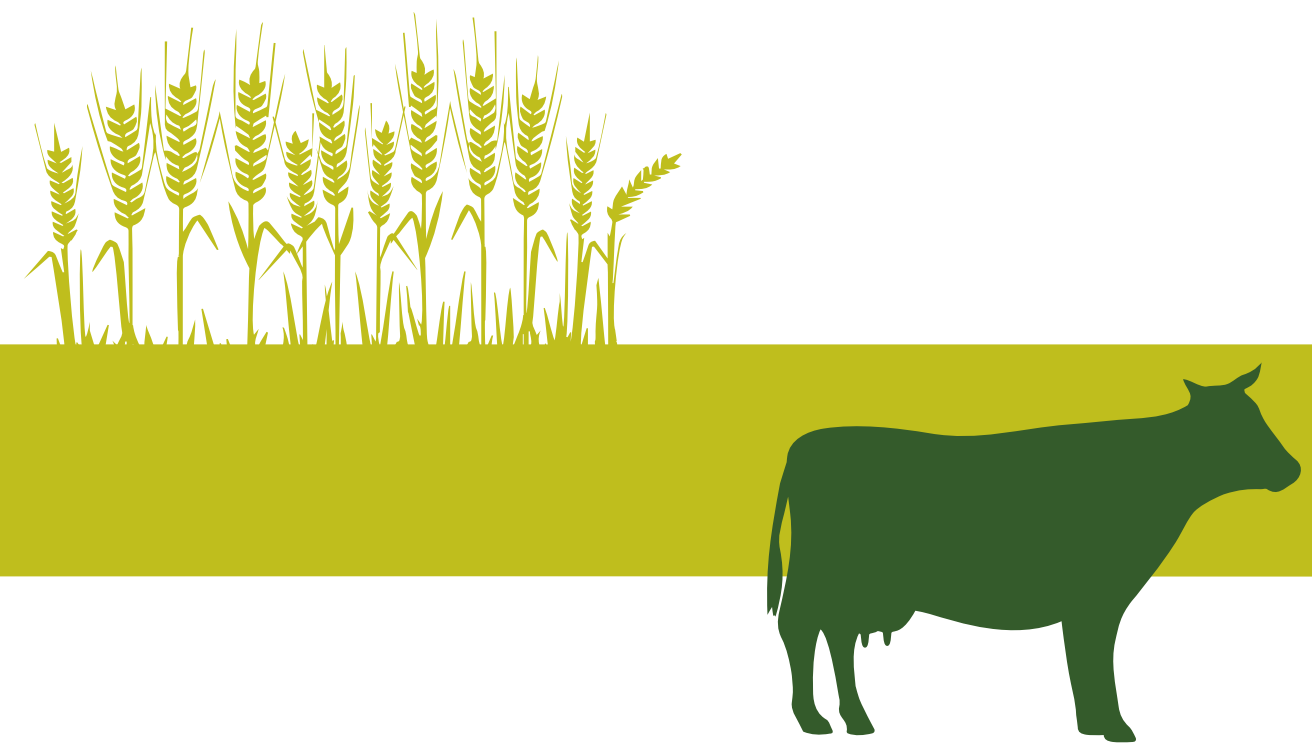

T/1 vdf Hochschulverlag AG an der ETH Zürich

(c) CC BY-N C-N D, https://vdf.ch/einfuhrung-in-die-schweizer-agrarpolitik-e-book.html 


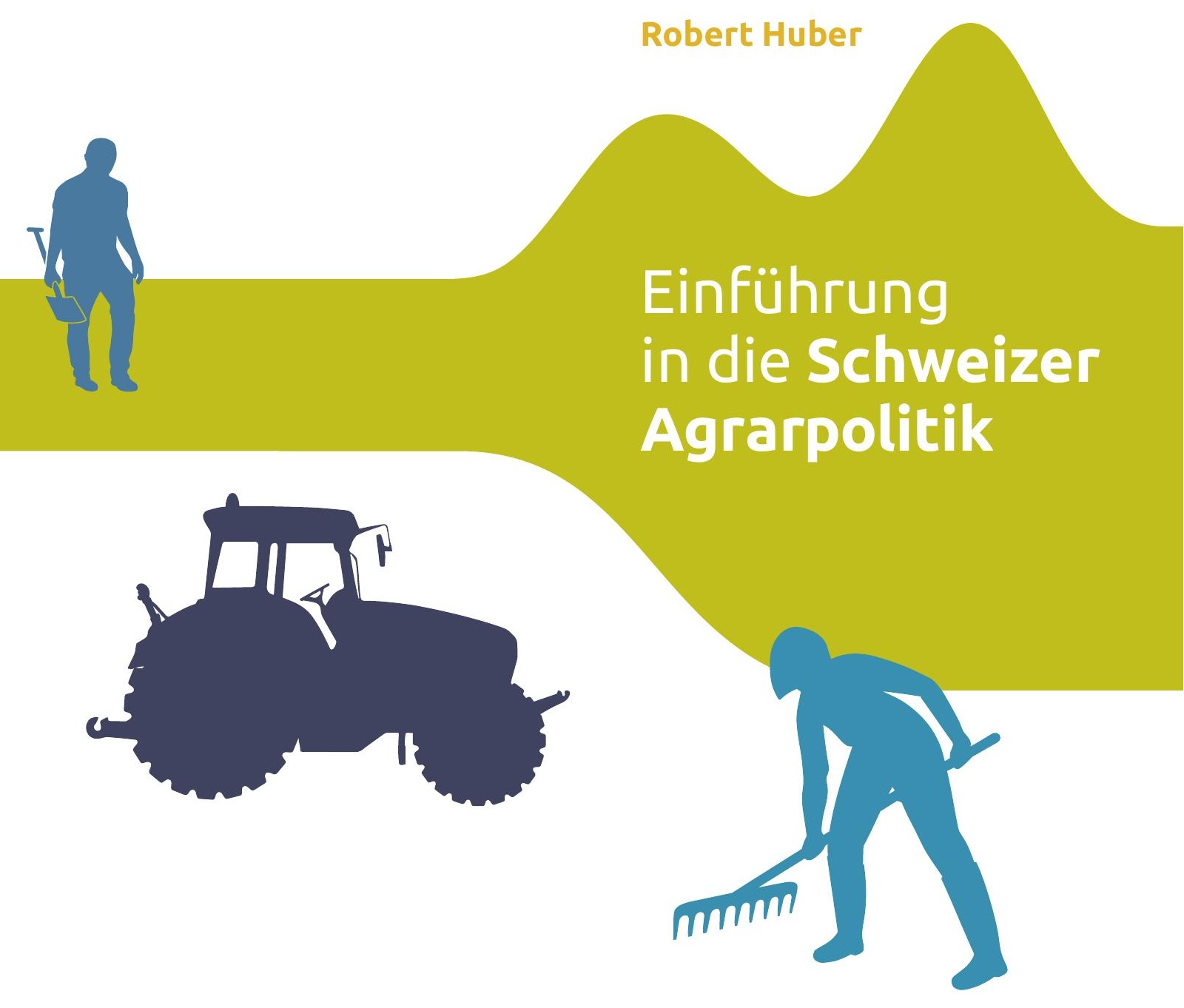

\section{DAECP}




\section{Bibliografische Information \\ der Deutschen Nationalbibliothek}

Die Deutsche Nationalbibliothek verzeichnet diese Publikation in der Deutschen Nationalbibliografie; detaillierte bibliografische Daten sind im Internet über http://dnb.dnb.de abrufbar.

ISBN 978-3-7281-4058-6 (Printausgabe)

Download open access:

ISBN 978-3-7281-4059-3/ DOI 10.3218/4059-3

www.vdf.ethz.ch

(c) 2022, vdf Hochschulverlag AG an der ETH Zürich

Das Werk einschliesslich aller seiner Teile ist urheberrechtlich geschützt. Jede Verwertung ausserhalb der engen Grenzen des Urheberrechtsgesetzes ist ohne Zustimmung des Verlages unzulässig und strafbar. Das gilt besonders für Vervielfältigungen, Übersetzungen, Mikroverfilmungen und die Einspeicherung und Verarbeitung in elektronischen Systemen.

Cover: Malte Belau

Foto: @ iStock.com/bluejayphoto

Getreide: ๑ istock.com/helenfoxgreen 


\section{Dank}

Dieses Buch ist aus der Vorlesung «Agrarpolitik» an der ETH Zürich hervorgegangen. Ohne die Unterstützung durch die Gruppe «Agrarökonomie und Agrarpolitik» wäre es jedoch nicht zustande gekommen. Ein spezieller Dank geht an Prof. Robert Finger, der das vorliegende Buch nicht nur ermöglichte, sondern deren Umsetzung immer konstruktiv unterstützte. Meinen Kolleginnen und Kollegen der obgenannten Gruppe danke ich für den intensiven und kritischen Austausch und für die vielen Inputs und Hinweise.

Darüber hinaus danke ich speziell Eva Lieberherr, Therese Haller, Christian Flury, Michael Hartmann, Martin Pidoux, Felix Schläpfer und Adrian Müller für ihre wertvollen Kommentare und Hinweise zu früheren Versionen des Buches. Ein grosser Dank geht auch an Angelika Rodlauer vom vdf Verlag. Sie hat das Buch sorgfältig redigiert, korrigiert und Unklarheiten ausgeräumt. Ein Dank geht auch an alle, die mir netterweise Bildmaterial oder Piktogramme zur Verfügung gestellt haben. Dazu gehören Katja Jacot, Roman Graf, Markus Jenny, Alain Farine und Alessandro Rossi. Schliesslich möchte ich mich auch ganz herzlich bei Prof. Peter Rieder und Prof. Bernard Lehmann bedanken. Ihre Arbeiten in Wissenschaft und Lehre an der ETH Zürich waren die Grundlagen für zahlreiche Konzepte und Theorien, die in diesem Buch behandelt werden.

Für verbliebene Fehler und Unklarheiten, für welche ich um Nachsicht bitte, übernehme ich die Verantwortung. Da die Agrarpolitik sowie die Vermittlung deren Inhalte einem stetigen Wandel unterworfen sind, wird die vorliegende Ausgabe in regelmässigen Abständen überarbeitet und neu aufgelegt werden. Ich hoffe, dass die «Einführung in die Schweizer Agrarpolitik» dadurch stets weiterentwickelt und nichts an ihrer Aktualität einbüssen wird. Ich wünsche den Leserinnen und Lesern, insbesondere den Studierenden meiner Vorlesung «Agrarpolitik», viel Freude bei der Lektüre. 


\section{Inhaltsverzeichnis}

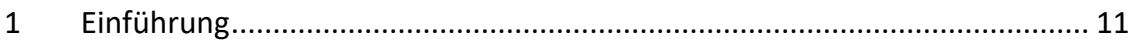

$1.1 \quad$ Die Eigenheiten des Agrarsektors .....................................................11

1.2 Die Bedeutung der Agrarpolitik im internationalen und nationalen Kontext.....................................................................................12

1.3 Ziele und Aufbau des Buches ..................................................................14

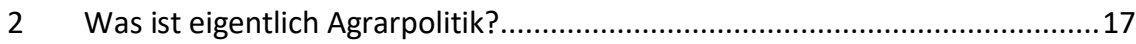

3 «Big picture»: Treiber der agrarpolitischen Entwicklung .................................25

3.1 Bevölkerungsentwicklung und Nahrungsmittelkonsum ........................26

3.2 Strukturelle Transformation der Wirtschaft .........................................28

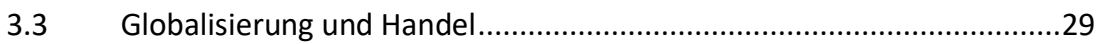

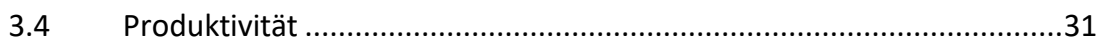

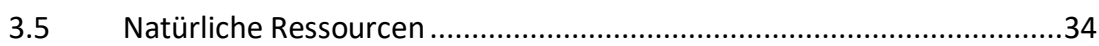

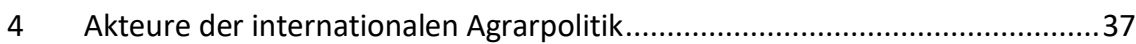

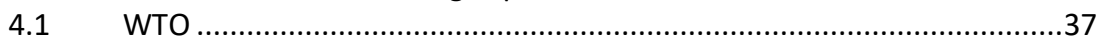

4.2 OECD (Organization for Economic Cooperation and Development) .........43

4.3 Food and Agriculture Organization of the United Nations, FAO .............47

4.4 Weitere Institutionen im Kontext der internationalen Agrarpolitik ........48

$5 \quad$ Konzeptionelle Grundlagen der Schweizer Agrarpolitik..................................51

$5.1 \quad$ Ziele und Zielkonflikte in der Agrarpolitik .........................................51

5.2 Grundlagen einer Agrarmarktordnung .............................................57

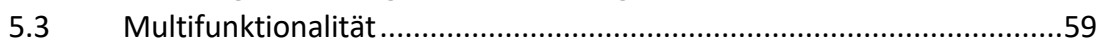

5.4 Ökonomische Grundlagen der Agrarumweltpolitik ...............................68

5.5 Beurteilung von Agrarpolitik: Wirksamkeit und Effizienz.........................73

$6 \quad$ Instrumente der Agrarpolitik (Policy) ........................................................79

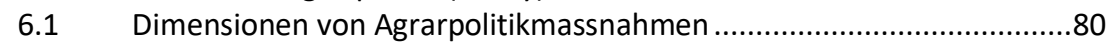

6.2 Wohlfahrtsanalytische Beurteilung von Politikmassnahmen .................85

$7 \quad$ Eine kurze Geschichte der Schweizer Agrarpolitik ........................................ 115

$7.1 \quad$ Ursprung der heutigen Agrarpolitik ..................................................115

7.2 Staatliche Ordnung nach den Weltkriegen .........................................116

7.3 Agrarpolitik für eine multifunktionale Landwirtschaft..........................118

7.4 Die Bedeutung der Volksinitiativen in der Entwicklung der Agrarpolitik .............................................................................120

7.5 Anstehende Weiterentwicklung der Agrarpolitik ...............................122

8 Erscheinungsbild der Landwirtschaft in der Schweiz ...................................125

$8.1 \quad$ Landwirtschaftliche Struktur ...........................................................125

8.2 Produktion und Wertschöpfung.....................................................126

8.3 Ausgaben des Bundes und der Haushalte...........................................129

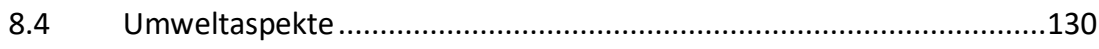




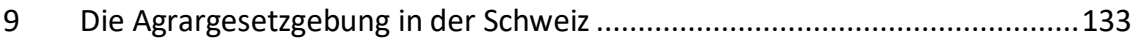

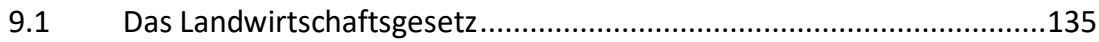

9.2 Bundesgesetz über das bäuerliche Bodenrecht.................................157

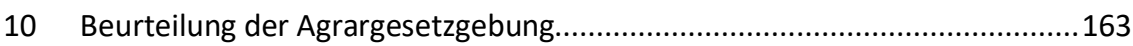

10.1 Grundbegriffe der Evaluation von Politikmassnahmen ......................163

10.2 Evaluationen der Schweizer Agrarpolitik ..............................................168

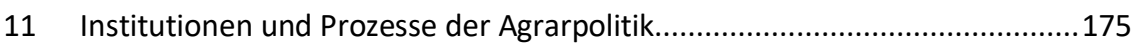

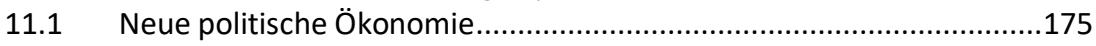

11.2 Der Politikzyklus ............................................................................186

11.3 Prozesse der Schweizer Agrarpolitik ...................................................188

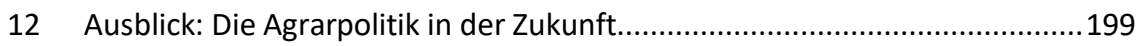

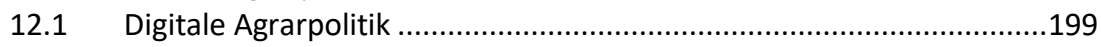

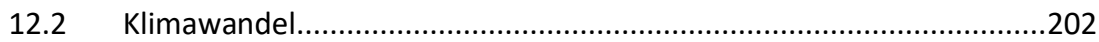

12.3 Von der Agrarpolitik zur Ernährungspolitik........................................204

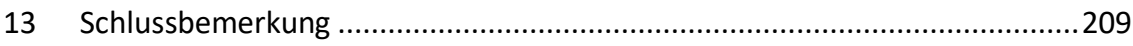

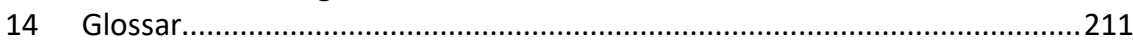

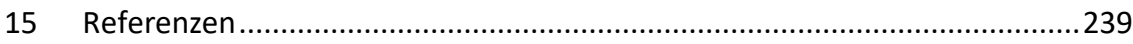




\section{Abbildungsverzeichnis}

Abbildung 1 Treiber der agrarpolitischen Entwicklung …..................................26

Abbildung 2 Der Verlust von Biodiversität durch die Abholzung von Regenwald .35

Abbildung 3 Zielkonflikte als zentrale Herausforderung der Agrarpolitik..............56

Abbildung 4 Die Biodiversitätsbeiträge in der Schweiz als typisches Beispiel für das Targeting und Tailoring von agrarpolitischen Massnahmen ........75

Abbildung 5 Dimensionen von agrarpolitischen Instrumenten über institutionelle und räumliche Ebenen.

Abbildung 6 Die Kernfrage in Bezug auf den Handel mit Agrarprodukten ist, wie das Regel- und Steuerungssystem unter der Berücksichtigung von Umwelt- und Sozialstandards ausgestaltet werden kann.

Abbildung 7 Politikmassnahmen können ungewollt dazu führen, dass negative Externalitäten über den Raum verschoben werden (Leakage).........105

Abbildung 8 Nach den Weltkriegen standen die Ernährungssicherheit, hohe Erträge und sichere Einkommen für die Bäuerinnen und Bauern im Zentrum der Agrarpolitik

Abbildung 9 Chronologie der Abstimmungen zur Schweizer Landwirtschaftspolitik seit 1896

Abbildung 10 Die Schweizer Landwirtschaft ist im europäischen Vergleich kleinstrukturiert.

Abbildung 11 Erscheinungsbild Schweizer Landwirtschaft 2019

Abbildung 12 übersicht über die Kernelemente der Gesetzgebung in der Schweizer Agrarpolitik

Abbildung 13 Karte der Schweizer Produkte mit einer Ursprungskennzeichnung AOP (1-21) und IGP (22-35)

Abbildung 14 Elemente des Policy-Cycles nach Ingold et al. (2016)..... 188

Abbildung 15 Die Veränderungen der Schweizer Agrarpolitik in den letzten 30 Jahren waren stark von der Verwaltung und dem Bundesrat geprägt.

Abbildung 16 Polity, Politics, Policy im Kontext der Schweizer Agrarpolitik 191

Abbildung 17 Koalitionen von privaten und öffentlichen Akteuren in der Entwicklung der Agrarpolitik 14-17 196

Abbildung 18 Die Digitalisierung der Landwirtschaft schafft auch neue Perspektiven für die Agrarpolitik 200

Abbildung 19 Der Klimawandel als doppelte Herausforderung für die Landwirtschaft 204

Abbildung 20 Elemente des Land- und Ernährungssystems 205 


\section{Verzeichnis der Boxen}

Box 1 Entwicklung des Producer Support Estimate PSE in der Schweiz................46

Box 2 Multifunktionalität der Landwirtschaft.......................................................60

Box 3 Die Konsumenten- und Produzentenrente .................................................66

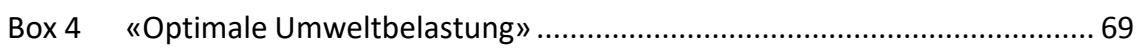

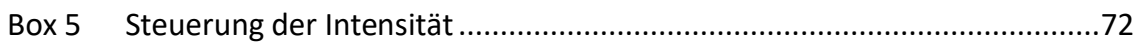

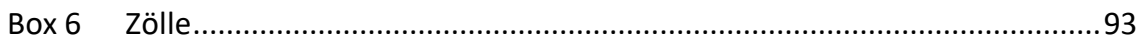

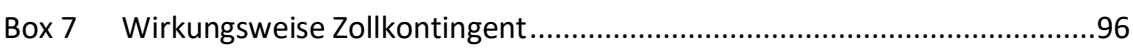

Box 8 Wirkungsweise Kontingent und Faktorsubvention .....................................99

Box 9 Wohlfahrtsgewinne durch die Internalisierung von positiven und negativen Externalitäten............................................................106

Box 10 Agrarreformetappen seit der Jahrtausendwende...................................119

Box 11 Bundesverfassung BV Art. 104 Landwirtschaft ....................................... 134

Box 12 Beispiel für eine Branchenorganisation: swiss granum...........................139

Box 13 Konzeptionelle Übersicht des Schweizer Direktzahlungssystem ...............145

Box 14 Beispielhaftes Wirkungsmodell zur Evaluation von Biodiversitätsbeiträgen BFF ....................................................................................166

Box 15 Organisation einer latenten Gruppe: Schweizer Bauernverband (SBV)....182

Box 16 Nachfrage nach privaten und öffentlichen Gütern .................................184 


\section{Einführung}

\section{READERS' GUIDE}

Warum gibt es eine Agrarpolitik und welche Bedeutung hat sie für die Gesellschaft im Allgemeinen und für Agronominnen und Agronomen im Speziellen? Diese kurze Einführung wirft ein Schlaglicht auf die Bedeutung der Agrarpolitik im nationalen und internationalen Kontext. Darüber hinaus werden in diesem Kapitel die Ziele und der Aufbau des Buches erläutert. Das Ziel dieser Einführung ist es, dass die Leserinnen und Leser dieses Buches wissen, warum und welche Inhalte vermittelt werden und wo sie welche Informationen finden können.

\subsection{Die Eigenheiten des Agrarsektors}

Die Land- und Ernährungswirtschaft sichert die Produktion von Nahrungsmitteln auf der ganzen Welt. Sie ist damit der zentrale Sektor für das Überleben und Wohlbefinden der Menschheit. Dabei unterscheidet sich der Agrarsektor von anderen Wirtschaftsbereichen durch eine Reihe von Charakteristiken (siehe z.B. Dabbert und Braun, 2012). Grundsätzlich lässt sich festhalten, dass Nahrungsmittel lebensnotwendig sind. Jede Bewohnerin und jeder Bewohner dieser Erde muss essen. Die Verfügbarkeit von Nahrungsmitteln und die Art, wie man sich ernährt, sind dabei nicht nur elementare Grundbedürfnisse, sondern auch Indikatoren für die gesellschaftliche Stellung und den Wohlstand einer Person (Pinstrup-Andersen, 2009). Die sogenannte Ernährungssicherheit oder «Food Security», d.h. die Verfügbarkeit und Bezahlbarkeit von sicheren Lebensmitteln, ${ }^{1}$ ist daher der Einstiegspunkt für fast alle agrarpolitischen Eingriffe in die Land- und Ernährungswirtschaft weltweit.

Neben der Unverzichtbarkeit auf Nahrungsmittel ist die Naturabhängigkeit der landwirtschaftlichen Produktion ein weiterer zentraler Aspekt des Agrarsektors. Die Erzeugung von Nahrungsmitteln ist bis heute dadurch gekennzeichnet, dass Boden, Wasser und Luft elementare Bestandteile des Produktionsprozesses sind, welche sogenannte Ökosystemleistungen für die Erzeugung von Nahrungsmitteln zur Verfügung stellen (Power, 2010). Auch der Umgang mit Tieren als Teil des Produktionspro-

\footnotetext{
${ }^{1}$ Definition by the United Nations: Food security exists when all people, at all times, have physical and economic access to sufficient safe and nutritious food to meet their dietary needs and food preferences for a healthy and active life.
} 
zesses ist ein Charakteristikum des Agrarsektors, welches ganz spezifische Herausforderungen im Bereich Ethik und Tierwohl verursacht. Daraus folgen verschiedene weitere Eigenschaften des Agrarsektors wie beispielsweise die bodenabhängige Produktion, d.h., der Boden ist nicht nur Standort, sondern auch Produktionsmittel. Die Abhängigkeit von der Umwelt bringt auch mit sich, dass saisonale Produktionskreisläufe entstehen, z.B. bedingt durch Jahreszeiten oder Reproduktionszyklus.

Mit der Abhängigkeit von variablen Ökosystemleistungen sind Produktionsrisiken verbunden. Wetterbedingte Schwankungen, aber auch Extremereignisse wie Trockenheit oder Frost führen zu saisonalen und räumlichen Schwankungen des Angebots von Nahrungsmitteln. Treffen diese auf eine unelastische Nachfrage, wir essen ja nicht mehr oder weniger in Abhängigkeit des Angebots, dann kann dies zu instabilen Märkten und hohen Preisfluktuationen führen.

Die Folge dieser landwirtschaftlichen Eigenschaften widerspiegeln sich auch in der Struktur und der Organisation des Agrarsektors. Im Gegensatz zu anderen Wirtschaftssektoren lassen sich aufgrund der Bodengebundenheit Skaleneffekte, d.h. eine effizientere Produktion durch Wachstum, nur bedingt umsetzen. Der Agrarsektor ist dadurch, im Gegensatz zu den übrigen Wirtschaftssektoren, klein strukturiert und bis heute weltweit durch Familienbetriebe geprägt (Graeub et al., 2016; Samberg et al., 2016; Meyfroidt, 2017). Gleichzeitig führen der technische Fortschritt und die strukturelle Transformation der Wirtschaft dazu, dass der Agrarsektor schrumpft und immer weniger Bäuerinnen und Bauern immer mehr Nahrungsmittel zur Verfügung stellen.

\subsection{Die Bedeutung der Agrarpolitik im internationalen und nationalen Kontext}

Diese spezifischen Charakteristiken des Agrarsektors führen dazu, dass politische Eingriffe in die Land- und Ernährungswirtschaft weltweit eine lange Tradition haben. Die Geschichte der Landwirtschaft kann dabei als «doppelte Abhängigkeit» beschrieben werden (Tauger, 2020). Damit ist gemeint, dass die Landwirtschaft einerseits mit der natürlichen Umwelt interagiert und von ihr abhängig ist. Andererseits stehen die Bäuerinnen und Bauern immer auch in einer komplexen Beziehung zur (städtischen) Zivilisation, die sie ernähren. Die nicht landwirtschaftliche Bevölkerung hatte schon in der Antike versucht die Landwirtschaft zu regeln, zu dominieren oder auszubeuten. Diese doppelte Anhängigkeit der Landwirtschaft, d.h. die Beziehungen von Umwelt Landwirtschaft und Landwirtschaft - Zivilisation, ist daher der Ursprung der Agrarpolitik seit der Antike (Tauger, 2020). 
Die grundlegenden Charakteristiken zeigen auch die Bedeutung des Agrarsektors für das Überleben der Menschheit. Der Agrarsektor und dessen Regelung durch die Agrarpolitik sind zentral für eine nachhaltige Entwicklung, d.h. eine langfristige Sicherstellung der ökonomischen, ökologischen und sozialen Ziele auf unserem Planeten. Das zweite von 17 Zielen für eine nachhaltige Entwicklung der UNO-Mitgliedstaaten bezieht sich dann auch direkt auf die Landwirtschaft: Beendigung des Hungers, Ernährungssicherheit und Zugang zu gesunden und sicheren Lebensmitteln sowie eine nachhaltige Landwirtschaft. Diese Ziele haben höchste Priorität für die nachhaltige Entwicklung weltweit (UN, 2016). Darüber hinaus beziehen sich auch andere Ziele der UNO direkt oder indirekt auf die Land- und Ernährungswirtschaft. ${ }^{2}$ Durch die Verbundenheit mit der Natur und der Gesellschaft und deren Interaktionen sind die Landwirtschaft und die Ernährung Teil unserer Lebenswelt, die weit über wirtschaftliche Aspekte hinausgeht und soziale Aspekte wie ländliche Entwicklung und kulturelles Erbe mit beinhaltet (z.B. Van Huylenbroeck et al., 2007).

Gleichzeitig spielt der Agrarsektor eine zentrale Rolle in der wirtschaftlichen Entwicklung von Ländern und Regionen (Timmer, 2009). Diese Wichtigkeit des Agrarsektors spiegelt sich in den Ausgaben verschiedener Länder für die Bäuerinnen und Bauern. In den OECD-Ländern werden jährlich über $\mathbf{5 0 0} \mathbf{M i a}$. US-Dollar für die Unterstützung der Landwirtschaft ausgegeben (OECD, 2020). Zudem ist die Agrarpolitik im Kontext des Welthandels und der Globalisierung ein zentraler Streitpunkt (siehe dazu das Kapitel 5 in diesem Buch).

In der Schweiz nimmt die wirtschaftliche Bedeutung des Agrarsektors laufend ab. Zugleich ist die Schweiz eines der Länder, welche die Landwirtschaft weltweit am stärksten unterstützt (OECD, 2015). Insgesamt sind es mehr als 4 Mia. CHF, die jährlich für die Landwirtschaft aus öffentlichen Mitteln ausgegeben werden (BLW, 2020). Dazu kommt die Stützung der Landwirtschaft über Zölle und andere Einfuhrbeschränkungen. Der Zusammenhang zwischen wirtschaftlicher Bedeutung und hoher Stützung ist keineswegs zufällig (Swinnen, 2010). Die Gründe werden in diesem Buch ausführlich besprochen. Viel wichtiger ist es an dieser Stelle festzuhalten, dass die Agrarpolitik für den Land- und Ernährungssektor in der Schweiz eine zentrale Rolle einnimmt. Es stellt sich in der Schweiz nicht die Frage, ob die Landwirtschaft unterstützt werden soll, sondern vielmehr wie. In der Schweiz tätige Agronominnen und Agronomen werden daher zwangsläufig mit Agrarpolitik in Berührung kommen, egal ob sie in der Wertschöpfungskette, der Beratung, der Schulung oder in Verbänden und

\footnotetext{
2 Promote inclusive and sustainable economic growth, employment and decent work for all (Ziel 8); Ensure sustainable consumption and production patterns (Ziel 12); Take urgent action to combat climate change and its impacts (Ziel 13) und Sustainably manage forests, combat desertification, halt and reverse land degradation, halt biodiversity loss (Ziel 15). Source: UN Sustainable Development Goals
} 
der Verwaltung tätig sind. Kenntnisse über die konzeptionelle Ausrichtung, die konkrete Ausgestaltung und der zukünftigen Weiterentwicklung der Agrarpolitik sind in allen Bereichen nützlich und in vielen Fällen sogar eine Voraussetzung, um zu einer nachhaltigen Entwicklung des Landwirtschafts- und Ernährungssektors in der Schweiz beitragen zu können.

\subsection{Ziele und Aufbau des Buches}

Das Ziel dieses Buches ist es, Basiswissen der Schweizer Agrarpolitik zu vermitteln, um Interessierten eine kritische Einordnung und Diskussion zu ermöglichen. Die Leserin und der Leser sollen die konzeptionellen Grundlagen, den Aufbau und die Wirkungsweise der Schweizer Agrarpolitik kennenlernen und verstehen, wie diese Agrarpolitik zustande kommt. Es werden agrarpolitische Zusammenhänge aus einer übergeordneten Perspektive eingeordnet und die Wirkungsweisen von agrarpolitischen Massnahmen und Instrumenten im Kontext der Schweizer Agrarpolitik kritisch beurteilt.

Diese Ziele werden in den einzelnen Kapiteln dieses Buches gespiegelt. Die Herausforderung ist dabei, dass die verschiedenen Ziele miteinander verknüpft sind. Die einzelnen Kapitel in diesem Buch beleuchten daher immer einen einzelnen Aspekt der Agrarpolitik. Jedes Kapitel hat dabei einen sogenannten "Readers' Guide», der am Anfang kurz einführt, worum es im jeweiligen Kapitel geht und wie dieses im Kontext der Agrarpolitik einzuordnen ist. Am Ende jedes Kapitels werden die wichtigsten Aspekte nochmals zusammengefasst. Auf diese Art entsteht für jedes Kapitel eine Art Klammer, die es ermöglichen soll, die einzelnen Kapitel auch unabhängig voneinander lesen zu können. Zusätzlich enthält das Buch ein Glossar, in welchem die wichtigsten Begriffe und Definitionen kurz erklärt sind. Dies sollte es dem Leser ermöglichen, auch zwischen den Kapiteln zu springen und trotzdem die wichtigsten Inhalte nachvollziehen zu können.

Das Buch beginnt mit einer Einordnung des Begriffs Agrarpolitik und wie dieser im vorliegenden Buch verwendet wird. Anschliessend folgen elf Kapitel, die sich in vier grundlegende Teile einordnen lassen. Der erste Teil, welcher das dritte und vierte Kapitel umfasst, beschäftigt sich mit dem Umfeld der Agrarpolitik in einem internationalen Kontext. Das dritte Kapitel fokussiert auf die globalen Treiber, d.h. diejenigen Einflussfaktoren, welche die Agrarpolitik weltweit beeinflussen. Das vierte Kapitel wirft dann ein Schlaglicht auf wichtige internationale Akteure im Kontext der Agrarpolitik. Dazu gehören insbesondere die Welthandelsorganisation (WTO), die Organisation für wirtschaftliche Zusammenarbeit und Entwicklung (OECD) und die Ernährungs- und Landwirtschaftsorganisation der Vereinten Nationen (FAO). 
Im zweiten Teil, der die Kapitel fünf und sechs beinhaltet, werden theoretische und konzeptionelle Grundlagen der Agrarpolitik beschrieben. Das bedeutet auch, dass die folgenden Kapitel sich nicht mehr einfach auf die ganze Welt übertragen lassen. Vielmehr wird hier ein spezifischer Fokus auf die Steuerung des Agrarsektors in Europa und der Schweiz geworfen. Im fünften Kapitel geht es dabei in erster Linie um Grundbegriffe und Definitionen. Das sechste Kapitel fokussiert auf die Darstellung von agrarpolitischen Instrumenten und deren wohlfahrtstheoretische Beurteilung.

Der dritte Teil mit den Kapiteln sieben bis zehn bezieht sich ausschliesslich auf die Schweizer Agrarpolitik. Das siebte Kapitel beinhaltet einen kurzen historischen Abriss der Schweizer Agrarpolitik. Kapitel acht fasst das heutige Erscheinungsbild der Schweizer Landwirtschaft kurz zusammen. Dieses Kapitel ist wichtig, um die Agrarpolitik und deren Massnahmen und Instrumente einordnen zu können. Dies erfolgt anschliessend im neunten Kapitel dieses Buches. Das zehnte Kapitel führt Begriffe der Politikevaluation ein und beschreibt eine Auswahl von Ergebnissen aus Evaluationen der Schweizer Agrarpolitik.

Der vierte Teil, das Kapitel elf, setzt den Fokus auf die Institutionen und Prozesse der Agrarpolitik. Das heisst, er beschäftigt sich mit der Frage, wie die Agrarpolitik zustande kommt. Das elfte Kapitel nutzt Konzepte aus der neuen politischen Ökonomie, um die Entwicklung der Agrarpolitik aus einer theoretischen Perspektive zu beschreiben. Dabei geht das Kapitel auch auf die spezifische Entwicklung der Schweizer Agrarpolitik der letzten zehn Jahre ein.

Im zwölften und abschliessenden Kapitel wird schliesslich ein Ausblick auf die Agrarpolitik der Zukunft gewagt. Auch wenn die Schweizer Agrarpolitik einem sehr dynamischen Prozess unterworfen ist, soll in diesem letzten Kapitel die Brücke geschlagen werden zu den anhaltenden Eigenheiten und Herausforderungen des Agrarsektors, die in der Einführung zu diesem Buch beschrieben wurden.

Das Buch dient als Grundlage für die Bachelorvorlesung "Agrarpolitik» an der ETH Zürich. Gewisse konzeptionelle Erläuterungen mögen daher, ohne Kenntnisse von mikroökonomischen und theoretischen Grundlagen, die in anderen Lehrveranstaltungen der ETH vermittelt werden, abstrakt erscheinen. Die Referenzen im Buch, welche am Ende jedes Kapitels aufgeführt werden, können helfen, mehr Informationen aus anderen Lehrbüchern oder weiteren wissenschaftlichen Quellen zu erhalten. Oft verwendete Begriffe werden im Glossar kurz erklärt. 


\section{ZUSAMMENFASSUNG}

Der Agrarsektor ist zentral für die Erhaltung der Menschheit. Der Steuerung des Agrarsektors durch die Politik kommt dadurch eine Schlüsselfunktion für die nachhaltige Entwicklung unseres Planeten zu. Die zentrale Frage dabei ist nicht, ob man den Agrarsektor regeln soll, sondern wie. Dieses Buch vermittelt Basiswissen über Agrarpolitik. Das Ziel ist es, dass die Leserinnen und Leser die konzeptionellen Grundlagen, den Aufbau und die Wirkungsweise der Schweizer Agrarpolitik kennen und verstehen, wie diese Agrarpolitik zustande kommt. Das Buch umfasst zwölf Kapitel, die sich in vier grundlegende Bereiche unterteilen: 1) die Einordnung der Agrarpolitik in einem internationalen Kontext; 2) die konzeptionellen und theoretischen Grundlagen der Agrarpolitik und deren Massnahmen und Instrumente; 3) die konkrete Beschreibung der Schweizer Agrarpolitik und 4) die Darstellung der Institutionen und Prozesse der Agrarpolitik anhand von Konzepten der neuen politischen Ökonomie.

\section{WEITERFÜHRENDE LITERATUR}

Das Buch von Mark B. Tauger Agriculture in world history fasst die Geschichte der Landwirtschaft von der Antike bis zum 21. Jahrhundert zusammen. Es illustriert die historische Beziehung zwischen Bäuerinnen und Bauern und der Gesellschaft in allen Weltregionen.

Für die betriebswirtschaftlichen Grundlagen sind z.B. das Lehrbuch von Stephan Dabbert und Jürgen Braun Landwirtschaftliche Betriebslehre oder dasjenige von Oliver Musshoff und Norbert Hirschauer Modernes Agrarmanagement: Betriebswirtschaftliche Analyse- und Planungsverfahren zu empfehlen. Letzteres wird im Rahmen des ETH-Agrarökonomiestudiums als Grundlage verwendet. 


\section{Was ist eigentlich Agrarpolitik?}

\section{READERS' GUIDE}

Die Agrarpolitik kann aus unterschiedlichen Blickwinkeln betrachtet werden. Je nachdem, ob man sie aus einer ökonomischen, soziologischen oder politikwissenschaftlichen Perspektive anschaut, können unterschiedliche Facetten beleuchtet werden. In diesem ersten Kapitel geht es darum, die inhaltliche Breite aufzuzeigen, die im Begriff Agrarpolitik steckt. Gleichzeitig sollen die Themen, welche im Rahmen dieses Lehrbuches ausführlich behandelt werden, hervorgehoben und eingeordnet werden. Das Lernziel dieses Kapitels ist es, dass die Leserinnen und Leser unterschiedliche Definitionen von Agrarpolitik und deren zentralen Herausforderungen kennen und den Begriff im Rahmen dieses Buches einordnen können.

Die Agrarpolitik ist im Grundsatz eine sektorale Wirtschaftspolitik. Das heisst, sie regelt und steuert den Landwirtschaftssektor grösstenteils mittels wirtschaftspolitischer Massnahmen. Sie unterscheidet sich dadurch beispielsweise von der Umweltschutzgesetzgebung, welche Querschnittsaufgaben über verschiedene Wirtschaftssektoren hinweg erfüllt (Ingold et al., 2016). Die Agrarpolitik in der Schweiz wird grundsätzlich auf Bundesebene gesteuert. Das heisst, dass der Bund (und nicht die Kantone oder Gemeinden) für die Gestaltung der Politik verantwortlich ist. Kantone haben mehrheitlich die Funktion, die Bundespolitik umzusetzen oder sie zu überwachen. Das schliesst nicht aus, dass nicht auch die Kantone eine aktive Agrarpolitik betreiben. Kantonale Agrarpolitiken sind in der Schweiz immer ergänzend zur Agrarpolitik des Bundes (Binder und Mann, 2019). Im Gegensatz beispielsweise zur Raumplanung kommen auch den Gemeinden nur wenig Funktionen zu (z.B. über die Ackerbaustellenleiter). Das Subsidiaritätsprinzip spielt in der bestehenden Agrarpolitik folglich eine untergeordnete Rolle.

Auch wenn die Agrarpolitik als sektorale Wirtschaftspolitik gilt, so weist sie doch viele Eigenschaften auf, die eine strikte Abgrenzung gegenüber anderen Politikbereichen schwierig macht. Dies spiegelt sich in unterschiedlichen Definitionen wider. So definiert beispielsweise das Bundesamt für Landwirtschaft (BLW) die Agrarpolitik als die Schaffung von Rahmenbedingungen für die Schweizer Bauernfamilien: «Mit der Agrarpolitik schafft der Bund geeignete Rahmenbedingungen, dass die Schweizer Bauernfamilien ihre Aufgaben für die Gesellschaft, wie sie in der Verfassung (Art. 104 BV) festgehalten sind, auch erfüllen können.» Der Schweizerische Bauernverband (SBV) 
definiert Agrarpolitik nicht nur als Wirtschaftspolitik, sondern auch ausdrücklich als Sozial- und Strukturpolitik: "Die Agrarpolitik regelt die Gestaltung der wirtschaftlichen, rechtlichen und sozialen Verhältnisse der Land- und Forstwirtschaft. Die tragenden Säulen der Agrarpolitik sind die Markt-, Sozial- und Strukturpolitik.»

Wissenschaftliche Definitionen der Agrarpolitik zielen eher auf ordnungspolitische Aspekte. Ordnungspolitik umfasst im weiteren Sinne alle staatlichen Massnahmen der Wirtschaftspolitik. Darüber hinaus bezeichnet Ordnungspolitik aber auch einen liberalen Denkansatz. Dieser besagt, dass die Wirtschaft in erster Linie nach den Prinzipien von Markt und Wettbewerb zu organisieren sei. Die Eigenverantwortung der Menschen soll vor dem staatlichen Handeln kommen. In diesem Sinne definierten die einflussreichen deutschen Agrarökonomen Henrichsmeyer und Witzke (1994) die Agrarpolitik: "Die Agrarpolitik umfasst die Gesamtheit aller Bestrebungen und Massnahmen, die darauf abzielen, die ordnungspolitischen Rahmenbedingungen für die Agrarwirtschaft zu gestalten und die agrarwirtschaftlichen Prozesse zu beeinflussen.» Auch die Schweizer Lehrbücher zur Agrarpolitik orientierten sich stark an einer wirtschaftlichen Definition. Peter Rieder, langjähriger Professor für Agrarwirtschaft an der ETH Zürich, lehrte in erster Linie Agrarmarktpolitik (Rieder und Anwander Phanhuy, 1994): "Agrarmarktpolitik umfasst die landwirtschaftliche Marktlehre einerseits und die Begründung sowie die Instrumente agrarpolitischer Eingriffe in das Marktgeschehen andererseits.» Die wirtschaftliche Perspektive auf die Agrarpolitik beinhaltete daher auch eine detaillierte Beschreibung der Agrarmarktordnungen, d.h. der staatlichen Eingriffe in die einzelnen Agrarmärkte (Rieder et al., 1992). Eine breitere Begrifflichkeit nutzte Ralph Anderegg in seinem Buch Grundzüge der Agrarpolitik (Anderegg, 1999): "Die Agrarpolitik ist eine sektorale Wirtschaftspolitik. Als politische Ökonomie der Landwirtschaft beschäftigt sie sich mit der Ordnung, den Strukturen und den Prozessen der Agrarwirtschaft, zudem mit den ökologischen, sozialen und kulturellen Aspekten.» Neuere Bücher fokussieren auf die Aspekte einer «nachhaltigen» Landwirtschaft und übernehmen daher einen ganzheitlichen Blick auf die Agrarpolitik wie beispielsweise Peter H. Feindt und Koautoren (2019) in ihrem Buch Ein neuer Gesellschaftsvertrag für eine nachhaltige Landwirtschaft oder John M. Antle und Srabashi Ray in ihrem Werk Sustainable Agricultural Development (2020).

Die Folgerung aus diesen Definitionen ist, dass es keinen abschliessenden Begriff für Agrarpolitik gibt. Wie man Agrarpolitik definiert, hängt davon ab, welche Fragestellung oder Forschungsinteressen man damit untersuchen möchte. In diesem Buch wird die Schweizer Agrarpolitik mithilfe von agrarökonomischen Konzepten verstanden und zu erklären versucht. Das bedeutet, dass der Fokus dieses Buches auf der Betrachtung der Agrarpolitik als sektorale Wirtschaftspolitik liegt. Das wiederum bedeutet aber nicht, dass ausschliesslich wirtschaftliche Themen betrachtet werden. Die unterschiedlichen Facetten der Agrarpolitik werden in diesem Buch immer wie- 
der aufgenommen und es wird ein Blick über den "wirtschaftlichen Tellerrand» geworfen. Drei Gründe sprechen für diese offene und breite Verwendung des Begriffs Agrarpolitik.

1) Die Ernährung und damit auch die landwirtschaftliche Produktion sind zentrale Elemente unserer Gesellschaft. Gleichzeitig interagiert die Landwirtschaft über die Produktionsgrundlagen Boden, Luft und Wasser mit der Umwelt. Die Steuerung des Wirtschaftssektors Landwirtschaft hat folglich immer auch Auswirkungen auf Gesellschaft und Umwelt. Es existiert dadurch keine scharfe Trennung zwischen Wirtschaft, Umwelt und Gesellschaft in der Agrarpolitik. Aus diesem Grund muss diese immer wieder aus einem interdisziplinären Blickwinkel betrachtet werden. Je nach Frage- und Problemstellung müssen unterschiedliche methodische Ansätze aus den Bereichen Betriebswirtschaft, Volkswirtschaft, Politikwissenschaften, Soziologie, Geschichte, Recht mit naturwissenschaftlichem Know-how wie Agronomie, Biologie oder Umweltsystemwissenschaften kombiniert werden. Das führt oft zu sehr komplexen Problemstellungen. Politische Entscheidungen sind folglich durch Güterabwägungen zwischen unterschiedlichen wirtschaftlichen, ökologischen und sozialen Zielen charakterisiert. Diese müssen oft unter Unsicherheit oder Nichtwissen getroffen werden. Eine rein wirtschaftliche Betrachtung der Agrarpolitik würde daher zu kurz greifen.

2) Die Agrarpolitik beschäftigt sich mit Problemen auf unterschiedlichen Skalen. Als Skalen werden vorwiegend Raum, Zeit und Institutionen verstanden. Innerhalb dieser drei Skalen werden unterschiedliche Levels unterschieden, die in all ihren Ausprägungen einen Bezug zur Agrarpolitik haben.

- $\quad$ Räumlich: Agrarpolitik hat einen Einfluss auf globale Entwicklungen wie Ernährungssicherheit, Hunger oder die Übernutzung der natürlichen Ressourcen. Gleichzeitig regelt die Agrarpolitik ebenso Auswirkungen auf der Ebene von einzelnen Parzellen wie beispielsweise im Bereich des Gewässerschutzes. Die Agrarpolitik beeinflusst dadurch sämtliche räumlichen Levels und oft steht etwas, das lokal sinnvoll erscheint, im Kontrast zu dem, was auf einem regionalen, nationalen oder sogar globalen Level zweckmässig wäre.

- Zeitlich: Die Agrarpolitik wird von Phänomenen beeinflusst, welche sich über sehr lange Zeiträume entfalten wie beispielsweise die demografische Entwicklung oder die Veränderung von sozialen Normen in der Gesellschaft. Gleichzeitig kann sich die Agrarpolitik aber auch mit den kurzfristigen Anpassungen von Verordnungen befassen, die eine direkte Auswirkung auf die landwirtschaftliche Produktion haben können. Durch dieses Zusammenwirken von Faktoren über zeitlichen Levels hinweg können Pfadabhängigkeiten entstehen, die oftmals über den einzelnen Sektor hinauswirken. 
- Institutionen: Institutionen bezeichnen Systeme, die über Verträge oder Regeln die Interaktionen zwischen Individuen sicherstellen. Dazu gehören nicht nur Regierungsorganisationen, sondern auch Märkte oder beispielsweise Bräuche. In der Agrarpolitik spielen Organisationen wie die Welthandelsorganisation WTO eine wichtige Rolle, da diese massgebliche Handelsregeln festlegen. Nationale und internationale Wertschöpfungsketten beeinflussen die Produktionsmöglichkeiten der Bäuerinnen und Bauern weltweit. Gleichzeitig wird die Agrarpolitik auch von kleinen Interessenorganisationen wie beispielsweise der bäuerlichen Gewerkschaft Uniterre mit beeinflusst. Diese können lokal und regional ganz wesentlich zu Veränderungen der ländlichen Gesellschaft beitragen.

3) Die Agrarpolitik beinhaltet exemplarisch ein Werturteilsproblem. Im Kern unterscheidet man zwischen Sachaussagen und Werturteilen. Sachaussagen beziehen sich auf die logische Herleitung von Hypothesen und deren empirischen Überprüfung. Werturteile dagegen bestimmen, ob etwas wünschenswert, gut, gerecht, akzeptabel oder abzulehnen ist. In diesem Sinne kann die Politik auch folgendermassen definiert werden (Lehmbruch zitiert in ZDA, 2020): «Sie [die Politik] ist gesellschaftliches Handeln, [...] welches darauf gerichtet ist, gesellschaftliche Konflikte über Werte verbindlich zu regeln.» Viele Konzepte der Agrarökonomie sind grundsätzlich in der Wohlfahrtsökonomie verankert. Kollektive Entscheidungsfindung kann aber nicht ausschliesslich auf wohlfahrtsökonomischen Überlegungen gründen (Sen, 2018). Agrarpolitik spiegelt dadurch zwangsläufig mehr als eine «sektorale Wirtschaftspolitik».

Nichtsdestotrotz hat dieses Buch zum Ziel, agrarökonomische Sachaussagen im Kontext eines interdisziplinären Verständnisses der Agrarpolitik zu vermitteln. Gerade im Wissen, dass sich Werturteile nicht einfach aus dem Kontext der Agrarpolitik ausschliessen lassen, sollen die hier vorgestellten Konzepte dazu beitragen, die Wertediskussion, d.h. das konstituierende Element der Agrarpolitik an und für sich, zu versachlichen. Die Idee dahinter ist, dass Sachaussagen ein wichtiger Baustein für die Entwicklung von Rahmenbedingungen für die Land- und Ernährungswirtschaft sind, die sich aber erst durch die politischen Prozesse und die Auseinandersetzung mit gesellschaftlichen Werten und Konflikten ergeben.

Es muss aber an dieser Stelle daraufhin gewiesen werden, dass die Herleitung von Sachaussagen von «ideologischen» Grundannahmen beeinflusst wird. Auch fundierte Grundlagen brauchen eine wissenschaftliche Diskussion über die zugrunde liegende Wertebeurteilungen (value judgments in Sen, 2018). Kritische Stimmen aus der Agrarsoziologie oder der Agrargeschichte sehen in der agrarökonomischen Perspektive auf die Agrarpolitik dann auch eine «neoliberale» Agenda, welche die Landwirtschaft dem Wachstumsparadigma des Kapitalismus unterwerfe (Potter und Tilzey, 2007; Weiss, 2017; Auderset und Moser, 2018). Die Diskussion darüber, ob die 
Agrarökonomie und Agrarpolitik der Schweiz tatsächlich als neoliberales Konzept definiert werden können, geht über den Inhalt dieser Publikation hinaus. Die Gleichsetzung von Agrarökonomie und Neoliberalismus zeigt aber exemplarisch die Herausforderung, die sich aus unterschiedlichen Wertehaltungen für die Beurteilung der Agrarpolitik ergibt.

Diese oben genannten drei Gründe, welche illustrieren, dass Agrarpolitik immer auch mehr ist als eine «sektorale Wirtschaftspolitik», lenken die Aufmerksamkeit direkt auf die Herausforderungen der Agrarpolitik in der heutigen Zeit: die Steuerung der Landwirtschaft bei unterschiedlichen gesellschaftlichen Werturteilen und Interaktionen von ökonomischen, ökologischen und sozialen Zielen über Institutionen Zeit und Räume hinweg. Langfristig wird die Agrarpolitik nicht darum herumkommen, sich von der Perspektive der sektoralen Politik zu lösen und integrale Lösungen für die Land- und Ernährungswirtschaft zu finden. Das würde bedeuten, dass die Agrarpolitik der Zukunft sich nicht nur mit der landwirtschaftlichen Produktion, sondern mit der ganzen Wertschöpfungskette von Nahrungsmitteln beschäftigen muss. Diese Aspekte werden z.B. auf der Ebene der EU diskutiert (Farm to Fork Strategy) und sind zentraler Bestandteil über die Zukunft der Agrarpolitik (De Schutter et al., 2020).

Für das Verständnis dieses Buches ist noch eine letzte Abgrenzung des Begriffs Agrarpolitik nötig. Um unterschiedliche Bereiche der Politik wie Form, Instrumente und Prozesse sprachlich voneinander zu trennen und gesondert betrachten zu können, wurden in den Politikwissenschaften die Begriffe Polity, Policy und Politics entwickelt (siehe auch Ingold et al., 2016, Kapitel 1.1). Die drei Begriffe lassen sich folgendermassen definieren:

- $\quad$ Polity umfasst die Form oder Struktur des Politischen und bezieht sich auf institutionelle Aspekte. Der Fokus ruht auf den verfassungsmässigen politischen Strukturen und Ordnungen einer Gesellschaft (Regierungssysteme, Parlamente, politische Parteien, internationale Organisationen, Interessenverbände etc.) und auf deren Rechtsordnung (Verfassung, Gesetze, Verordnungen etc.), welche die Handlungsspielräume, Zuständigkeiten und Abläufe bei Auseinandersetzungen festlegen. Neben der institutionellen Ebene (Staatskunde) ist die normative Ebene (Wertvorstellungen, Absichten hinter Regelungen) angesprochen.

- $\quad$ Policy beinhaltet die Inhalte politischer Auseinandersetzungen, es geht um die Gegenstände, Aufgaben und Ziele, welche die Beteiligten formulieren und realisieren wollen (Gesetze, Verordnungen, Programme etc.). Somit zielt diese Dimension auf Problemlösung und auf die Gestaltung gesellschaftlicher Verhältnisse und ist Ausdruck von Interessen- und Zielkonflikten. 
- $\quad$ Politics fokussiert auf Prozesse, wie politische Verfahren (z.B. Gesetzgebungsprozesse, Abstimmungen, Lobbyismus), und auf die Konfliktanalyse bzw. darauf, wie Interessengruppen ihre Anliegen durchzusetzen suchen. Untersucht wird der Willens- und Entscheidungsbildungsprozess (politische Auseinandersetzungen, Debatten etc.) der am politischen Geschehen Beteiligten.

Inhaltlich fokussiert dieses Buch auf die Aspekte der Policy, das sind die Ziele und Instrumente der Agrarpolitik und wie diese eingesetzt werden, sowie auf den Aspekt der Politics, d.h. auf das Verständnis der zentralen politischen Prozesse der Schweizer Agrarpolitik. Aspekte der Polity hingegen werden nicht oder nur am Rande behandelt. Das bedeutet aber nicht, dass diese Ebene nicht auch wichtig für die Agrarpolitik ist. Im Gegenteil, es existiert im internationalen Kontext eine umfangreiche Forschung zur Interaktion zwischen Staatsformen und der Agrarpolitik z.B. in China, Russland oder den Entwicklungsländern (Swinnen, 2018). Mit dem Fokus dieses Buches auf die aktuelle Schweizer Agrarpolitik spielen aber die verfassungsmässigen Strukturen eine untergeordnete Rolle. Die direkte Demokratie und die Grundlagen der Verfassung werden hier als gegeben betrachtet.

\section{ZUSAMMENFASSUNG}

Die Agrarpolitik ist im Grundsatz eine sektorale Wirtschaftspolitik. Sie wirkt sich stark auf Umwelt und Gesellschaft aus. Die Agrarpolitik legt die Regeln fest, unter welchen die landwirtschaftliche Produktion funktioniert. Die zentrale Herausforderung in der Steuerung der Landwirtschaft ist die Berücksichtigung der Interaktionen von ökonomischen, ökologischen und sozialen Zielen über Institutionen, Zeit und Räume hinweg. Dabei treffen im politischen Prozess unterschiedliche gesellschaftliche Werturteile über die Frage, was wünschenswert, gut oder gerecht ist, aufeinander. Aufgrund der Interaktionen von Wirtschaft, Umwelt und Gesellschaft über verschiedene Skalen hinweg wird sich die Agrarpolitik wohl langfristig in eine ganzheitliche Politik der Land- und Ernährungswirtschaft (Food Policy) entwickeln müssen. Dieses Buch fokussiert aber auf die bestehende Agrarpolitik und versucht mithilfe von agrarökonomischen Konzepten die Politiken (Policies) und deren Prozesse (Politics) in der Schweizer Agrarpolitik zu erklären und zu verstehen.

\section{WEITERFÜHRENDE LITERATUR}

Lesenswert ist das Buch von John M. Antle und Srabashi Ray Sustainable Agricultural Development: An Economic Perspective. Dieses Buch fasst die Herausforderungen für die Landwirtschaft und die Agrarpolitik in verschiedenen Regionen der Welt aus einer agrarökonomischen Perspektive zusammen. 
Das Buch von Peter Feindt, zusammen mit verschiedenen Koautorinnen und Koautoren, mit dem Titel Ein neuer Gesellschaftsvertrag für eine nachhaltige Landwirtschaft erläutert anschaulich die agrarpolitischen Herausforderungen des Landwirtschaftssektors aus einer deutschen und europäischen Perspektive.

Als Grundlage für die Begrifflichkeiten aus den Politikwissenschaften eignet sich das Lehrbuch von Karin Ingold, Eva Lieberherr, Isabelle Schläpfer, Kathrin Steinmann und Willi Zimmermann mit dem Titel Umweltpolitik der Schweiz, welches ebenfalls an der ETH als Lehrbuch verwendet wird. 


\section{3 «Big picture»: Treiber der agrarpoliti- schen Entwicklung}

\section{READERS' GUIDE}

Die Ausgestaltung der Agrarpolitik unterscheidet sich stark zwischen Weltregionen und dem wirtschaftlichen Entwicklungsstand eines Landes. Trotzdem gibt es Einflussfaktoren, die sich überall auf der Welt manifestieren und in der Agrarpolitik gespiegelt werden. Dazu gehört die strukturelle Transformation der Wirtschaft und Gesellschaft, die Bevölkerungsentwicklung und die Veränderung der Nachfrage nach Nahrungsmitteln, die Globalisierung, die Entwicklung der landwirtschaftlichen Produktivität über technischen Fortschritt und Innovation sowie die natürlichen Grenzen der landwirtschaftlichen Produktion. Diese globalen Aspekte werden im Folgenden vorgestellt. Das Ziel dieses Kapitels ist es, dass die Leserinnen und Leser den Zusammenhang zwischen globalen Treibern und der Agrarpolitik erkennen und diese zeitlich und in Bezug zu unterschiedlichen Ländern und Regionen und deren Agrarsektoren einordnen können.

Im letzten Kapitel wurde ausgeführt, wie wichtig verschiedene räumliche, zeitliche und institutionelle Skalen und die Verbindung mit anderen Wirtschaftssektoren für die Agrarpolitik sind. Das bedeutet, dass die grossen und globalen Entwicklungen auch die Agrarpolitik mit beeinflussen. Dabei stehen fünf Treiber im Fokus (Abbildung 1). Wichtig ist anzumerken, dass die Agrarpolitik natürlich nicht nur beeinflusst wird von diesen globalen Entwicklungen, sondern dass die Agrarpolitik selbst versucht, diese Treiber zu beeinflussen.

Aus einer agrarökonomischen Perspektive können die Treiber im Kontext der Entwicklungen des Angebots und der Nachfrage sowie deren Ausgleich auf den Märkten dargestellt werden. Auf der Seite der Nachfrage sind es die demografische Entwicklung der Bevölkerung und deren Nahrungsmittelkonsum, die sich auf den Agrarsektor und deren Regelung auswirken. Auf der Seite des Angebots steht die Veränderung der produzierten Nahrungsmittel im Zentrum, d.h., die Steigerung der landwirtschaftlichen Produktivität ist eine wichtige Ursache für die Gestaltung der Agrarpolitik. 


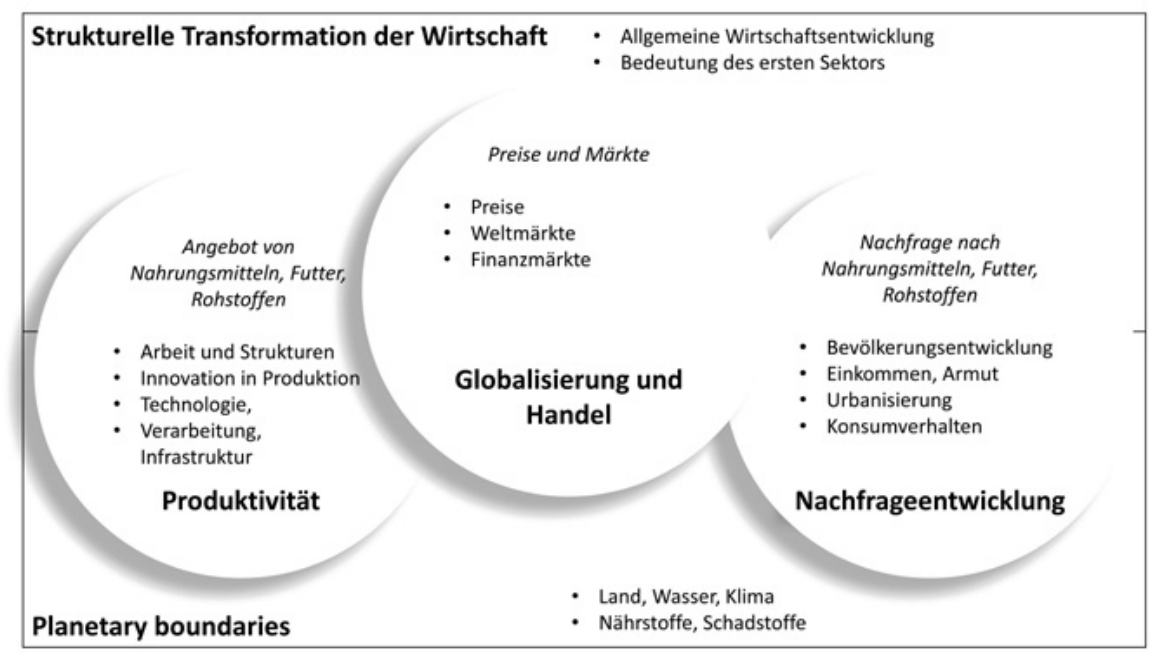

Abbildung 1. Treiber der agrarpolitischen Entwicklung: Strukturelle Transformation, Produktivität, Nachfrageentwicklung, Globalisierung und Handel sowie die Nutzung der natürlichen Ressourcen und deren begrenzte Verfügbarkeit (in Anlehnung an Von Braun, 2015).

In der Regelung von Angebot und Nachfrage über Märkte spielt die Globalisierung der Wertschöpfungsketten und des Handels eine zentrale Rolle. Die Grundlage der landwirtschaftlichen Produktion sind natürliche Ressourcen wie Boden oder Wasser. Die Nutzung dieser Ressourcen ist mit positiven und negativen Externalitäten verbunden, die in vielen Fällen den Charakter von öffentlichen Gütern aufweisen. Die natürlichen Grenzen (Planetary Boundaries), die durch die Agrarökosysteme vorgegeben sind, sind dadurch ein weiterer wichtiger Aspekt in der Entwicklung der Agrarpolitik. Schliesslich kommt auch der strukturellen Transformation der Wirtschaft, d.h. der Entwicklung von der Agrar- zur Dienstleistungsgesellschaft, eine wichtige Rolle in der Agrarpolitik zu. In den folgenden Unterkapiteln werden diese fünf Treiber ausführlich erläutert.

\subsection{Bevölkerungsentwicklung und Nahrungsmittelkonsum}

Die Versorgung mit Nahrungsmitteln ist seit Anbeginn der Menschheit der Schlüssel zu deren Überleben. Die Kontrolle über Land und Produktionsmittel war der Ursprung von Entwicklung, Wohlfahrt und Macht. Fehlende Nahrungsmittel oder ein Mangel an lebenswichtigen Nährstoffen führen zu Hunger, Krankheit und Tod. Die Grenzen und Möglichkeiten, die Versorgung der Bevölkerung mit Lebensmitteln sicherzustellen, liefern daher oft eine wichtige Erklärungsgrundlage für den historischen Aufstieg von Gesellschaften und Nationen (siehe dazu z.B. Diamond, 2014). Die Steuerung der landwirtschaftlichen Produktionsfaktoren - also die Agrarpolitik - wird 
daher grundlegend von der Bevölkerungsentwicklung und deren ausreichende Versorgung beeinflusst. Das Phänomen des "Land Grabbing», d.h. der staatliche Kauf von landwirtschaftlichem Boden in anderen Ländern, bekam in diesem Kontext viel Aufmerksamkeit (De Schutter, 2011). Landesgrenzen beschränken die Staaten/Nationen nicht mehr, für eine ausreichende Versorgung ihrer Bevölkerung zu sorgen.

Zentral ist dabei, dass die Bevölkerung des Planeten im Verlauf der letzten 150 Jahre exponentiell gewachsen ist. Auch wenn die Wachstumsrate mittlerweile wieder rückläufig ist, gehen Prognosen davon aus, dass die Weltbevölkerung bis 2050 auf 10 Mia. Menschen anwachsen wird. Der Grossteil dieses Bevölkerungswachstums wird in Entwicklungs- und Schwellenländern in Afrika und Asien stattfinden. Eine zentrale Herausforderung für die Agrarpolitik ist es daher, diese Menschen ausreichend mit Nahrungsmitteln zu versorgen, ohne die natürlichen Produktionsgrundlagen wie Boden, Wasser oder natürliche Ökosysteme komplett zu zerstören (Godfray et al., 2010).

Wie schwierig das wird, kann durch die Tatsache illustriert werden, dass heute noch mehr als 800 Mio. Menschen oder ca. 13 \% der Weltbevölkerung unterernährt sind (UN, 2020). Der Trend war anfangs des Jahrtausends über längere Zeit rückläufig, d.h., der Anteil der Unterernährten an der gesamten Weltbevölkerung nahm ab. Seit ein paar Jahren und verstärkt durch die COVID-Krise nimmt der Anteil aber relativ und in absoluten Zahlen wieder zu. Die Ziele der internationalen Gemeinschaft zur Reduktion von Hunger und Armut im Rahmen der sogenannten «Sustainable Development Goals» werden daher nur teilweise erfüllt und es bleibt offen, ob das langfristige Ziel von $6 \%$ bis 2030 erreichbar ist (Hickel, 2016). Gleichzeitig wird geschätzt, dass heute ca. $\mathbf{5 0 0}$ Mio. Menschen übergewichtig sind (Hasegawa et al., 2019). Der Einfluss der Bevölkerungsentwicklung und Nachfrage nach Nahrungsmitteln auf die Agrarpolitik ist folglich nicht rein arithmetischer Natur (Anzahl Kalorien x Anzahl Menschen). Ganz entscheidend sind auch die Kaufkraft und die Ernährungsgewohnheiten in den verschiedenen Regionen der Welt. Während Hunger ursächlich mit Armut zusammenhängt, verändern sich mit zunehmendem Wohlstand die Ernährungsgewohnheiten. Reichere Nationen konsumieren beispielsweise mehr Fleisch. Zurzeit wächst die weltweite Wirtschaftsleistung und führt indirekt zu einem Anstieg des Konsums von tierischen Produkten (Tilman und Clark, 2014).

Ein weiterer Aspekt, der die Agrarpolitik nachfrageseitig beeinflusst, sind die Nahrungsmittelverluste entlang der Wertschöpfungskette, d.h. Food Waste (z.B. Alexander et al., 2017). In vielen Entwicklungsländern mit disaggregierten Produktionssystemen sind die Verluste auf dem Feld durch Schädlinge oder Krankheiten, im Transport, der Lagerung und Verarbeitung von hoher Bedeutung. In den Industrieund Schwellenländern hingegen ist ein Grossteil der Verluste auf die Ansprüche der Verarbeitung und Konsumenten zurückzuführen. Nahrungsmittel werden weggeworfen, weil sie nicht den Produktionsnormen für die Weiterverarbeitung entsprechen 
oder weil der Konsument keine Sorgfalt im Umgang mit Menge und Zubereitung von Nahrungsmitteln walten lässt.

Die Kombination von wachsender Weltbevölkerung, sich verändernden Ernährungsmustern und dem Verlust von vielen Kalorien entlang der Wertschöpfungskette führt dazu, dass die Nachfrage nach Nahrungsmitteln bis in absehbarer Zukunft zunimmt. Diese Zunahme der Nachfrage nach Lebensmitteln war und ist einer der fünf Treiber für die Entwicklung und Ausgestaltung der Agrarpolitik weltweit.

\subsection{Strukturelle Transformation der Wirtschaft}

In der Wirtschaftslehre wird die Landwirtschaft, gemeinsam mit der Waldwirtschaft, als Primärsektor von industriellen Tätigkeiten (2. Sektor) und dem Dienstleistungssektor (3. Sektor) abgegrenzt. Noch vor knapp 150 Jahren war der überwiegende Teil der arbeitenden Bevölkerung der Schweiz in der Land- und Forstwirtschaft beschäftigt (Haller, 2011). Mit der Industrialisierung der Wirtschaft nahm dieser Anteil stetig ab. Mittlerweile ist eine Mehrheit der Bevölkerung im Dienstleistungssektor tätig. Diese Transformation, die mit einer Steigerung der wirtschaftlichen Leistung (gemessen am Bruttosozialprodukt) einhergeht, ist ein globales Phänomen. Überall auf der Welt hat sich mit zunehmender Wirtschaftsleistung der Anteil der im ersten Sektor beschäftigten Bevölkerung reduziert, weil die Produktivitätssteigerung ausserhalb der Landwirtschaft grösser war als innerhalb des Sektors (Pull-Faktor). Daraus folgt, dass je reicher eine Nation, desto tiefer der Anteil der Landwirtschaft an der Wirtschaftsleistung ist und desto weniger Beschäftigte im Agrarsektor sind. Diese Reduktion der Anzahl Beschäftigten im ersten Sektor wird zudem durch eine gesteigerte landwirtschaftliche Produktivität verstärkt (Push-Faktor). Weltweit war die Überwindung von Armut immer mit dieser strukturellen Transformation der Wirtschaft verbunden und aus einer historischen Perspektive war sie der Pfad aus der Armut (Timmer, 2009).

Für die Landwirtschaft resultiert aus der Entwicklung hin zur Dienstleistungsgesellschaft ein strukturelles Problem. Normalerweise verläuft der Rückgang des Anteils der Landwirtschaft an der Wirtschaftsleistung (am Bruttosozialprodukt) schneller als die Reduktion der Anzahl Beschäftigten im Sektor. Eine wirtschaftstheoretische Logik impliziert jedoch, dass die Produktionsfaktoren (Arbeit, Kapital, Boden) sich so lange verschieben, bis die Produktivität in den verschiedenen Sektoren gleich sind und alle Produktionsfaktoren zu ihrem Grenzprodukt entlohnt werden (z.B. Mankiw et al., 1992). Praktisch bedeutet dies, dass im Vergleich mit anderen Wirtschaftssektoren in der Landwirtschaft im Durchschnitt tiefere Einkommen erzielt werden. Der Grund dafür ist, dass die Landwirtschaft einen höheren Anteil der Bevölkerung beschäftigt, als er anteilsmässig zum Bruttosozialprodukt beiträgt. Tiefe Löhne in den landwirtschaftlichen Betrieben sind eng mit diesem strukturellen Grundproblem verbunden. 
Insbesondere in ländlichen Gebieten mit einer grossen Bedeutung der Landwirtschaft übt dieser strukturelle Effekt einen enorm hohen Druck aus (IFPRI, 2019). Wenn man die Einkommen allein als Indikator für die wirtschaftliche Leistung der Landwirtschaft nimmt, dann kann nur mit einer Reduktion der Beschäftigten oder einem Anstieg der Wertschöpfung diesem wirtschaftlichen Druck auf die Landwirtschaft und die ländlichen Regionen entgegengewirkt werden. Die Schweiz zeigt exemplarisch die Herausforderung, die aus der strukturellen Transformation hervorgeht. Der Anteil der Landwirtschaft am Bruttosozialprodukt liegt noch bei 0,6\%. Nach wie vor sind aber mehr als $4 \%$ der Bevölkerung (gemessen in Vollzeitäquivalenten) in der Landwirtschaft tätig. Die Löhne sind entsprechend tief - auch im Vergleich zu Branchen mit ähnlichem Kapital- oder Arbeitseinsatz. Wichtig ist in diesem Kontext zu erwähnen, dass die Messung des landwirtschaftlichen Einkommens keineswegs trivial ist. Die Entwicklung des Kapital- und Bodenwertes in der Landwirtschaft ist beispielsweise äusserst komplex und eine Abgrenzung von Haushalt und verschiedenen Einkommensbestandteilen wie Nebenerwerb oder Kapitaleinkommen ist schwierig (Poppe und Vrolijk, 2019).

Der wirtschaftliche Druck, der sich aus der strukturellen Transformation ergibt, ist ein wesentlicher Treiber für die Entwicklung und Ausgestaltung der Agrarpolitik. Die Entwicklung hin zur Dienstleistungsgesellschaft wird die wirtschaftliche Bedeutung der Landwirtschaft auch in Zukunft verringern. Eine Welt, in welcher es in den entwickelten Ländern keine Landwirtschaft mehr gibt, wird es in absehbarer Zeit aber nicht geben. Zu wichtig ist die Nahrungsmittelproduktion für das Funktionieren von Gemeinschaften, Gesellschaften und Nationen. Unter dem enormen wirtschaftlichen Druck stellt sich aber die Frage, auch in der Schweiz, wo wir produzieren, wie viel und zu welchem Preis (Timmer, 2009).

\subsection{Globalisierung und Handel}

Die ökonomische Logik des Handels ist, dass komparative Kostenvorteile von Handelspartnern genutzt werden können, um die Wohlfahrt für beide zu erhöhen (z.B. Frey, 1985). Die Steigerung der wirtschaftlichen Leistung durch Handel ist unbestritten. Nationen, die offen sind für den Austausch von Gütern und Dienstleistungen, sind prinzipiell reicher, d.h., sie haben ein höheres Bruttosozialprodukt und höhere durchschnittliche Einkommen (OECD 2020 online). Aus einer ökonomischen Sicht ist auch der Handel mit Agrargütern wohlfahrtssteigernd (z.B. Anderson und Martin, 2005). In den Verhandlungen im Rahmen des General Agreements on Tariffs and Trade (GATT) und der daraus entstandenen World Trade Organization (WTO) war und ist der Handel mit landwirtschaftlichen Produkten ein zentrales Element (siehe nächstes Kapitel). Die weltweite Arbeitsteilung und die damit verbundene Globalisierung macht vor der Landwirtschaft also nicht halt (Robinson, 2018). 
Die Handelsvolumen sind in den letzten Jahrzehnten im Nahrungsmittelbereich gestiegen (Krausmann und Langthaler, 2019). Der Handel mit landwirtschaftlichen Produkten ist dabei durch eine hohe Komplexität gekennzeichnet (Puma et al., 2015). Diese ergibt sich aus einer Vielzahl von Akteuren und Produkten auf dem Weltmarkt. Zwei Aspekte machen den Handel mit Agrarprodukten besonders attraktiv. Erstens unterscheiden sich die Produktionsbedingungen wie Böden, Klima oder Topografie in den unterschiedlichen Regionen der Welt massiv. Kaffee, Kakao, Wein oder Früchte sind typische Produkte, die weltweit konsumiert werden, jedoch nur in bestimmten Zonen überhaupt angebaut werden können. Zweitens erlaubt es die saisonale Natur der landwirtschaftlichen Produktion, Produktionsrisiken, z.B. Ernteausfälle in einer Region, über Handelsverträge mit anderen Regionen abzusichern. Diese räumliche und saisonale Heterogenität macht Agrarprodukte zu attraktiven Handelsgütern, unabhängig von komparativen Kostenvorteilen. Aus diesem Blickwinkel lässt sich auch verstehen, weshalb die ersten Produkte, die an einer Börse gehandelt wurden (Chicago Board of Trade), landwirtschaftliche Produkte wie z.B. Weizen und Baumwolle waren (Williams, 1982). Mithilfe von Börsentransaktionen konnten räumliche und zeitliche Angebotsknappheiten (z.B. Schweinezyklus) ausgeglichen werden. Ein agrarpolitischer Eingriff in den Handel von Agrarprodukten kann daher auch Auswirkungen auf weit entfernte Regionen haben. Dies wird heute mit dem Begriff Telecoupling umschrieben (Friis und Nielsen, 2018).

Die grossen Unterschiede in den räumlichen und zeitlichen Möglichkeiten der Nahrungsmittelproduktion implizieren aber nicht wirtschaftliche Opportunitäten, sondern in vielen Fällen auch eine ökologische Notwendigkeit. Das globale Bevölkerungswachstum erfolgt räumlich sehr unterschiedlich (siehe oben). Würden alle Nahrungsmittel vor Ort bzw. innerhalb einer Nation produziert, würden die natürlichen Ressourcen in bevölkerungsreichen Ländern zwangsläufig übernutzt (Haberl et al., 2007). Würde man die Versorgung beispielsweise auf einen Radius von 100 km beschränken, könnten gerade mal knapp $30 \%$ der wichtigsten pflanzenbasierten Nahrungsmittel nachhaltig gedeckt werden (Kinnunen et al., 2020). Ohne Handel würden sich auch die negativen Effekte des Klimawandels verstärken, weil weniger Ausgleich zwischen den unterschiedlich betroffenen Regionen möglich wäre (Janssens et al., 2020). In einer freiheitlichen und marktwirtschaftlich orientierten Welt ist Handel aus ökonomischer, ökologischer und sozialer Sicht daher essenziell. Die entscheidende Frage aus agrarmarktpolitischer Sicht ist daher nicht, ob Handel mit Agrarprodukten überhaupt stattfinden soll, sondern unter welchen Bedingungen und Regeln.

Trotz des steigenden Handels und der damit verbundenen Gewinne ist die Menge der gehandelten Agrarprodukte im Vergleich zur insgesamt produzierten Menge klein. Ein Beispiel dafür ist der weltweite Reismarkt. Nur gerade $9 \%$ des weltweit produzierten Reises, insgesamt ca. 500 Mio. t, werden gehandelt. Das bedeutet, dass Reis vorwiegend dort produziert wird, wo er auch konsumiert wird. Auch Mais und 
Weizen weisen mit einem Anteil von 15 bzw. $24 \%$ an der total produzierten Menge tiefe Handelsvolumen auf (AMIS, 2020). Die Handelsvolumen für tierische Produkte sind ebenfalls tief. Weltweit werden nur gerade $15 \%$ des Rindfleisches, $9 \%$ des Schweinefleisches und ungefähr $10 \%$ des Geflügelfleisches gehandelt (AMIS, 2020). Man spricht in diesem Zusammenhang von «dünnen Märkten» (Thin Markets). Diese implizieren, dass geringe Verschiebungen von Angebot oder Nachfrage zu Preissprüngen führen können (Puma et al., 2015). Sie sind daher durch eine hohe Volatilität charakterisiert (Piot-Lepetit und M'Barek, 2011). Volatilität auf Agrarmärkten ist ein wichtiger Treiber in der Entwicklung von agrarpolitischen Massnahmen. Viele Instrumente sind dazu gedacht, die Preisvolatilität auf den Agrarmärkten abzuschwächen. Die Volatilität von Marktpreisen und die Nahrungsmittelsicherheit sind die zentralen Argumente für die Rechtfertigung von agrarpolitischen Massnahmen. Gleichzeitig ist aber die Agrarpolitik in vielen Fällen auch der Verursacher von Volatilität. Während der Lebensmittelkrise 2008-2009 beispielsweise verfügten viele Länder einen Stopp der Exporte. Dadurch stiegen die Preise auf den dünnen landwirtschaftlichen Märkten noch stärker an (Watson, 2017).

Schliesslich haben sich in der Logik der wirtschaftlichen Globalisierung auch die landwirtschaftlichen Wertschöpfungsketten internationalisiert (De Schutter, 2017; Saitone und Sexton, 2017). Im Bereich der vor- und nachgelagerten Wertschöpfungsstufen der Landwirtschaft hat eine Marktkonzentration stattgefunden. Die Marktanteile der Unternehmen im Bereich Saatgut oder Pflanzenschutz konzentrieren sich auf wenige international tätige Firmen (Schimmelpfennig et al., 2004; Deconinck, 2020). Auch im Detailhandel fand eine Marktkonzentration statt (Hovhannisyan et al., 2018). Die Schweiz, mit ihrem geschützten Agrarmarkt, zeigt diese Entwicklung exemplarisch vor. In einem abgeschlossenen Wirtschaftsraum konzentrieren sich die Unternehmen auf wenige Akteure wie Migros, Coop oder Fenaco. Diese für den landwirtschaftlichen Sektor spezifischen Formen der Marktkonzentration und die damit verbundenen Asymmetrien in der Marktmacht sind ebenfalls ein wichtiger Faktor, der die Ausgestaltung der Agrarpolitik beeinflusst. Es ist wichtig, an dieser Stelle darauf zu verweisen, dass die Globalisierung per se in den meisten Ländern dieser Welt kein Ziel der Agrarpolitik ist. Durch die Einbettung in die gesamte gesellschaftliche und wirtschaftliche Entwicklung kann sich die Landwirtschaft und die Agrarpolitik aber nicht den Kräften der Globalisierung entziehen (siehe dazu auch die Ausführungen im Kapitel 6.3).

\subsection{Produktivität}

Ein zentraler Aspekt hinter der Entwicklung der Agrarpolitik ist die Produktivitätsentwicklung im landwirtschaftlichen Sektor. Analog zur Preisvolatilität auf den Agrarmärkten ist die Rolle der Agrarpolitik im Bereich der Produktivitätssteigerung gegen- 
seitig. Einerseits zielen gewisse agrarpolitische Instrumente darauf ab, negative Auswirkungen der Produktivitätssteigerung abzufedern. Andererseits existiert eine Vielzahl von Instrumenten, welche die Produktivität in der Landwirtschaft fördern.

Grundsätzlich wird unter Produktivität das Verhältnis von Output zu Input verstanden, also beispielsweise produzierte Kalorien pro Arbeitsstunde oder pro Flächeneinheit. Die Produktivitätssteigerung in der Landwirtschaft war historisch betrachtet durch verschiedene Revolutionen geprägt: über die Domestizierung der Nutztiere und Pflanzen vor Tausenden von Jahren zur Einführung von Fruchtfolgen und landwirtschaftlichen Praktiken vor ein paar hundert Jahren und zur sogenannten Grünen Revolution in den Siebziger- und Achtzigerjahren des letzten Jahrhunderts (Khush, 2001; Godfray et al., 2010). Der Begriff «Grüne Revolution» fasst die systematischen Formen der Züchtung und den gezielten Einsatz von externen Hilfsstoffen wie Pflanzenschutzmittel und Kunstdünger in der Landwirtschaft zusammen. Diese Grüne Revolution brachte eine nie dagewesene Steigerung der landwirtschaftlichen Produktion. Die Produktion von Getreide konnte bei gleichem Input um $300 \%$ gesteigert werden. Im tierischen Bereich lag der Produktionsfortschritt bei Geflügel beispielsweise bei mehr als $400 \%$. Diese historisch einmalige Ausdehnung der Produktion erlaubte es, rein rechnerisch zumindest, dass die Nahrungsmittelproduktion mit dem Bevölkerungswachstum mithalten konnte (Tilman et al., 2002). Der britische Ökonom Malthus prophezeite 1798 in seinem «Essay on the Principle of Population", dass die landwirtschaftliche Produktion sich nur linear ausdehnen könne und daher Hungersnöte eine zwingende Folge des Bevölkerungswachstums sei, wurde dadurch widerlegt.

Analog zu den diversen Produktionsbedingungen waren aber auch die Produktivitätsfortschritte durch die Grüne Revolution gänzlich ungleich verteilt (Alston und Pardey, 2014). In den meisten entwickelten Ländern stieg zuerst sowohl die Arbeits- wie auch die Flächenproduktivität an. In Gebieten mit knapper Fläche wie beispielsweise der Schweiz oder Japan, die bereits hohe Erträge aufwiesen, stieg in der Folge nur noch die Arbeitsproduktivität als Folge der zunehmenden Mechanisierung an. In Ländern mit viel Fläche und tiefen Erträgen wie beispielsweise Australien blieben die Erträge relativ tief und der Fortschritt bezog sich hauptsächlich auf die Arbeitsproduktivität. Im Gegensatz dazu stieg die Arbeits- und Flächenproduktivität in Entwicklungsländern kaum an. Diese Unterschiede sind aber nicht direkt auf unterschiedliche landwirtschaftliche Strukturen zurückzuführen. Der Output an Kalorien pro Kopf und Tag korreliert nicht mit der Betriebsgrösse in den jeweiligen Ländern. Länder mit Grossbetrieben wie Australien, den USA oder Argentinien produzieren nur unwesentlich mehr Kalorien pro Kopf als Länder mit kleinen Betriebsstrukturen wie beispielsweise Japan, Südkorea oder Mexiko. Nach wie vor sind daher auch kleine Familienbetriebe das Rückgrat der landwirtschaftlichen Produktion weltweit. Von den global ca. 570 Mio. Landwirten ist der allergrösste Teil den Familienbetrieben zuzurechnen. Diese bewirtschaften rund 75 \% der Flächen weltweit. Davon besitzen aber ca. 450- 
500 Mio. weniger als 2 ha Land (Eastwood et al., 2010; Lowder et al., 2016; Samberg et al., 2016).

Diese kleinen Strukturen können, in Kombination mit Produktivitätsfortschritten, negative Auswirkungen auf die Landwirtschaft haben. Ein Effekt hängt damit zusammen, dass Bäuerinnen und Bauern aufgrund ihrer atomistischen Struktur sogenannte Mengenanpasser sind. Das heisst, sie können im Markt nicht den Preis beeinflussen, sondern nur ihre Produktionsmenge den gegebenen Marktpreisen anpassen. Auf Produktivitätsfortschritte folgte meist eine Ausweitung des Angebots. Solange sich die Nachfrage nicht im gleichen Ausmass anpasst, sinken dadurch die Agrarpreise. Die Landwirtschaft hat dadurch den Anreiz, die Produktion erneut auszudehnen, um den Erlösrückgang durch höhere Produktionsmengen auszugleichen. Dieser Teufelskreis von Produktivitätsfortschritt, Mengenausdehnung und Preisrückgang wurde in der Literatur als «Agricultural Treadmill» beschrieben (Cochrane, 1958; Levins und Cochrane, 1996). Empirisch ist aber der Effekt sehr viel komplizierter (Meyfroidt et al., 2018). In der Realität waren die Preisentwicklungen von verschiedenen weiteren Faktoren beeinflusst. Richtig ist aber, dass der Trend der Agrarpreise, abgesehen von Krisensituationen, über das ganze letzte Jahrhundert nach unten zeigte. In diesem Kontext wurde auch das sogenannte "Farm Problem» als Mechanismus vorgeschlagen. Dieses besagt, dass das Angebot von und die Nachfrage nach Nahrungsmitteln kurzfristig eher unelastisch sind, langfristig das Angebot aufgrund des technischen Fortschritts aber schneller wächst als die Nachfrage (Gardner, 1992). Auch mit diesem Erklärungsansatz müssten die Preise für landwirtschaftliche Produkte kontinuierlich sinken. Der Trend sinkender Agrarpreise prägte das gesamte 20. Jahrhundert. In den letzten 20 Jahren haben verschiedene Ereignisse wie beispielsweise die Nahrungsmittelkrise 2008-2009 oder steigende Nachfrage nach Agrarprodukten und agrarischem Biodieseln diesen Trend etwas abgeschwächt.

Darüber hinaus können sich Preissenkungen positiv auf die nicht landwirtschaftliche Bevölkerung auswirken. Im Gegensatz zu den Bäuerinnen und Bauern profitieren beispielsweise die Konsumenten von kurzfristig tiefen Preisen. In Gebieten, in denen die arme Bevölkerung nicht mehr in den landwirtschaftlich geprägten ländlichen Regionen, sondern in der Stadt leben, führen tiefere Nahrungsmittelpreise zu einer Verbesserung der Nahrungsmittelsicherheit (FAO, 2011). Die fortlaufende Urbanisierung in den Entwicklungsländern führt dazu, dass in manchen Fällen sinkende und nicht steigende Nahrungsmittelpreise zur Bekämpfung des Hungers notwendig sind.

Gleichzeitig steht eine neue Agrarrevolution vor der Tür. Neue Methoden im Bereich der Züchtung wie z.B. CRISPR/Cas (Eş et al., 2019) und neue Informations- und Kommunikationstechnologien (ICT) (Basso und Antle, 2020) könnten der Landwirtschaft zu einem nächsten Produktivitätssprung verhelfen, der, im besten Fall, zu einer ressourcenschonenden Landwirtschaft führen könnte. Im schlechtesten Fall könnten diese neuen Zuchtmethoden und die Digitalisierung aber auch einer industrialisierten 
Landwirtschaft Vorschub leisten, die keine Rücksicht auf eine nachhaltige Nutzung der natürlichen Ressourcen nimmt. Hier offenbart sich die aktuelle Herausforderung für die Agrarpolitik (Walter et al., 2017).

\subsection{Natürliche Ressourcen}

Ein zentrales Charakteristikum der Landwirtschaft ist, dass sie inhärent mit der Nutzung von (Agrar-)Ökosystemen verbunden ist. Die Landwirtschaft nutzt Nährstoffe im Boden, Wasser und die Energie des Sonnenlichts als zentrale Produktionsfaktoren. Auch andere sogenannte Ökosystemleistungen ("Ecosystem Services») wie die Bestäubung durch Bienen oder der Erosionsschutz durch Grasland liefern der Landwirtschaft unabdingbare Produktionsgrundlagen (Heal und Small, 2002). Gleichzeitig werden durch die landwirtschaftliche Nutzung von natürlichen Ökosystemen eine Vielfalt von weiteren Leistungen für die Gesellschaft bereitgestellt. Dazu gehören beispielsweise die Pflege der Kulturlandschaft oder die Erhaltung der Biodiversität (Power, 2010).

Die natürlichen Produktionsgrundlagen sind jedoch begrenzt. Intensive landwirtschaftliche Produktionsformen können zu einer Übernutzung der Agrarökosysteme führen (Tilman et al., 2011). Die Folge sind negative Auswirkungen wie Nitrat im Grundwasser, Treibhausgasemissionen in der Atmosphäre, Antibiotika in der Tierhaltung oder Pflanzenschutzmittel in Oberflächengewässer. Forschende gehen davon aus, dass die Grenzen dessen, was die Erde langfristig aushalten kann («Planetary Boundaries»), in vielen Bereichen bereits erreicht worden sind (Steffen et al., 2015). Insbesondere der Stickstoffeintrag in die Ökosysteme und der Verlust an genetischer Diversität haben die tragbaren Grenzen bereits überschritten; beides Bereiche mit einer direkten Verbindung zur Landwirtschaft (Kanter et al., 2020a; Kanter et al., 2020b; Leclère et al., 2020).

Ursprünglich spielten die Ökosysteme in der Agrarpolitik nur insofern eine Rolle, als dass diese zur Nutzung für die landwirtschaftliche Produktion verfügbar gemacht wurden. Dazu gehörten beispielsweise grosse Gewässerkorrekturen, Meliorationen oder Güterzusammenlegungen. In den letzten Jahren wurden jedoch die positiven Externalitäten und öffentlichen Güter, welche durch die Landwirtschaft bereitgestellt werden, zu einer zentralen Rechtfertigung der Agrarpolitik. Hinzu kommt, dass der Klimawandel die natürlichen Produktionsgrundlagen und damit die Produktivität der Landwirtschaft stark verändert hat (Ortiz-Bobea et al., 2020) und es auch in Zukunft tun wird (Stevanović et al., 2016). Damit werden die Biodiversität und Ökosystemleistungen weiter unter Druck kommen (Mooney et al., 2009). Der Schlüssel zu einer nachhaltigen Landwirtschaft liegt damit im Umgang mit den natürlichen Ressourcen und den komplexen sozioökonomischen und ökologischen Trade-offs, die dadurch entstehen können (Antle und Ray, 2020). 


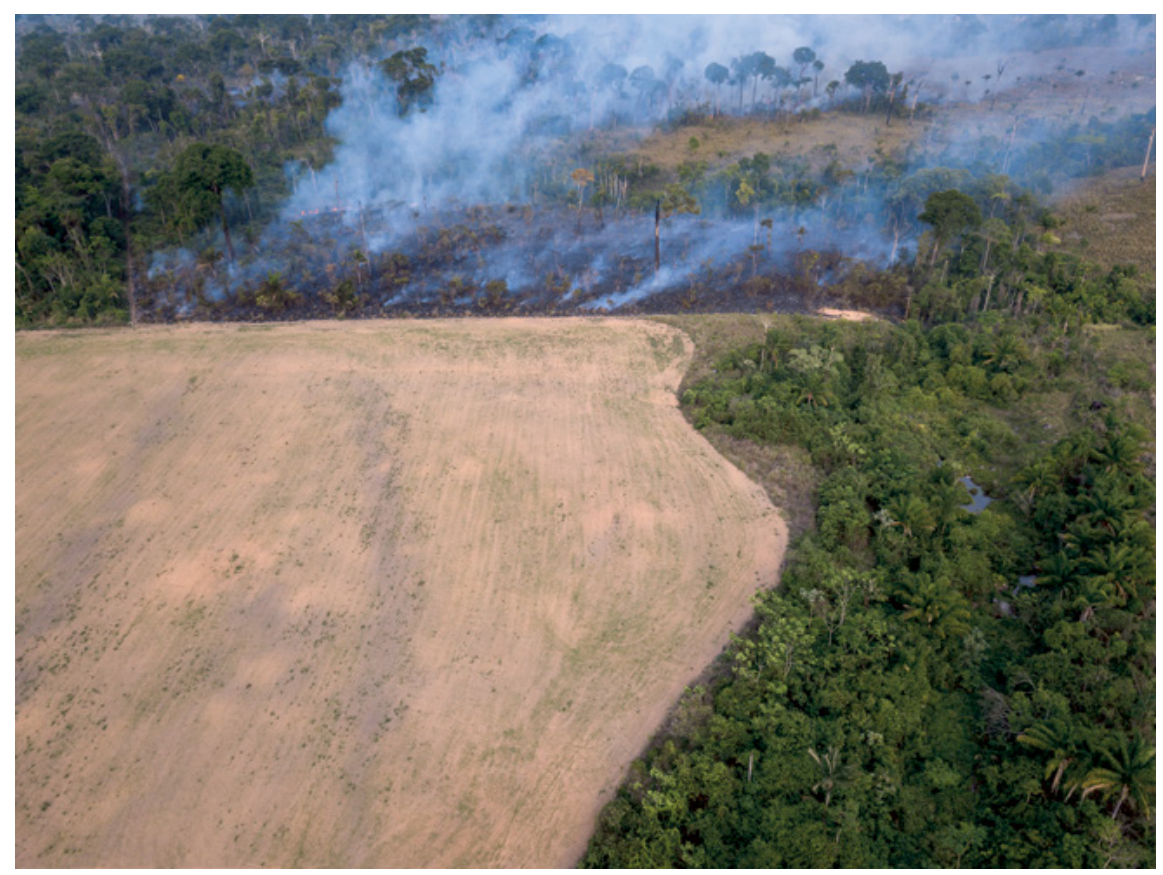

Abbildung 2. Die Intaktheit der Biosphäre gehört zu denjenigen «Planetary Boundaries», die gemäss Experten langfristig nicht mehr sichergestellt werden können. Im Bild der Verlust von Biodiversität durch die Abholzung von Regenwald für die landwirtschaftliche Nutzung in Para, Brasilien. (Bild: Paralaxis/iStock)

\section{ZUSAMMENFASSUNG}

Die Agrarpolitik wird von globalen Trends mit beeinflusst. Die strukturelle Transformation der Wirtschaft hin zu einer Dienstleistungsgesellschaft setzt die Landwirtschaft und die ländlichen Regionen weltweit unter Druck. Dazu kommt die Veränderung der Nachfrage nach Nahrungsmitteln, die wiederum durch das Bevölkerungswachstum und die Anpassung der Ernährungsgewohnheiten getrieben ist. Die Frage, wie die Weltbevölkerung ernährt werden kann, ist die Schlüsselfrage für die Agrarpolitik weltweit. In diesem Kontext spielen die Globalisierung der Wertschöpfungskette und der Handel mit Agrarprodukten eine wichtige Rolle. Handel per se ist eine Notwendigkeit für eine nachhaltige Landwirtschaft. Mit der zunehmenden Arbeitsteilung entstehen aber auch Konzentrationsprozesse, die sich langfristig negativ auswirken könnten. Die Agrarpolitik muss hier Bedingungen und Regeln festlegen. Die Entwicklung der Produktivität in der Landwirtschaft (Faktor-, Flächen- und Kapitalproduktivität) ist ein weiterer wichtiger Treiber für die Ausgestaltung der Agrarpolitik. Die Folgen von stetig zunehmenden Produktivitätssteigerungen sind ambivalent. 
Einerseits erlauben sie eine ressourceneffizientere Produktion. Andererseits erhöhen sie den Druck auf kleine landwirtschaftliche Strukturen, welche nach wie vor das Rückgrat der weltweiten Produktion bilden. Die Kombination von Nachfrageänderungen, Globalisierung und Produktivitätsfortschritten führte dazu, dass die landwirtschaftlichen Preise im letzten Jahrhundert tendenziell gesunken sind. Gleichzeitig nahm der Druck auf die natürlichen Produktionsgrundlagen wie Böden und Wasser zu. Stickstoffeinträge, Treibhausgasemissionen, Pflanzenschutzmittel und der Antibiotikaeinsatz sind drängende Probleme in der Landwirtschaft. Die Agrarpolitik wird von diesen Treibern aber nicht nur beeinflusst, sondern versucht die Auswirkungen dieser Entwicklungen auch zu beeinflussen und zu steuern.

\section{WEITERFÜHRENDE LITERATUR}

Ein Text, der die strukturelle Transformation der Landwirtschaft eindrücklich zusammenfasst, ist das kleine Buch von Peter C. Timmer: A World Without Agriculture:

The Structural Transformation in Historical Perspective.

Der Übersichtsartikel von Guy M. Robinson in der Zeitschrift Annual Review of Resource Economics mit dem Titel «Globalization of Agriculture» zeichnet ein ausgewogenes Bild von Vor- und Nachteilen der Globalisierung im Kontext der Landwirtschaft.

Mit Blick auf die begrenzten Ressourcen ist das populärwissenschaftliche Buch von Paul Collier eine interessante Quelle: Der hungrige Planet. Das Buch fokussiert nicht auf Landwirtschaft, wirft aber einen Blick auf die Frage, wie sich Ökologie und Ökonomie in Zeiten einer wachsenden Weltbevölkerung und der Klimaerwärmung zusammenführen lassen.

Einen ganzheitlichen Blick auf die Treiber der Agrarpolitik wirft Urs Niggli in seinem Buch Alle satt? Ernährung sichern für 10 Milliarden Menschen. 


\section{Akteure der internationalen Agrarpolitik}

\section{READERS' GUIDE}

Globale Treiber, welche die Landwirtschaft vor Herausforderungen stellen, führen auch dazu, dass auf internationaler Ebene nach Regelungen gesucht wird. Mit Blick auf die Agrarpolitik stehen dabei drei internationale Organisationen im Fokus: die Welthandelsorganisation, die Organisation für wirtschaftliche Zusammenarbeit und Entwicklung und die Organisation für Nahrungsmittel und Landwirtschaft der Vereinten Nationen. Diese drei Organisationen werden im folgenden Kapitel vorgestellt. Das Ziel dieses Kapitels ist es, dass die Leserinnen und Leser die unterschiedlichen Aufgaben und Wirkungsbereiche der Organisationen kennenlernen. Darüber hinaus sollen die Verbindung und Bedeutung für die Schweizer Agrarpolitik beleuchtet werden.

Für das Verständnis der Schweizer Agrarpolitik ist es wichtig, die internationalen Institutionen zu kennen, die sich aktiv in die Entwicklung, das Monitoring und die Evaluation von agrarpolitischen Massnahmen einbringen. Dazu gehören die Welthandelsorganisation (World Trade Organization, WTO), die Organisation für wirtschaftliche Zusammenarbeit und Entwicklung (Organization for Economic Cooperation and Development, OECD) und die Organisation für Nahrungsmittel und Landwirtschaft der Vereinten Nationen (Food and Agriculture Organization, FAO).

\subsection{WTO $^{3}$}

Die WTO ist die internationale Organisation für Handelsfragen mit Sitz in Genf. Sie wurde am 1. Januar 1995 gegründet. Mittlerweile sind 164 Länder Mitglied in der WTO (von weltweit insgesamt 193 existierenden Staaten). Das Ziel der WTO ist die Liberalisierung des internationalen Handels durch Abbau von Handelshemmnissen und Zöllen.

\subsubsection{Entstehung der WTO ${ }^{4}$}

Nach dem 2. Weltkrieg wurden in Bretton Woods die Weltbank und der Internationale Währungsfonds gegründet. Diese sollten auf einer globalen Ebene den Zugang zu Kapital sicherstellen und die wirtschaftliche Entwicklung weltweit fördern. Diese

\footnotetext{
${ }^{3}$ Weitere Information zur WTO: www.wto.org.

${ }^{4}$ Dieser Abschnitt beruht auf den Ausführungen von Senti, R., 2000. WTO. System und

Funktionsweise der Welthandelsordnung. Schulthess, Zürich.
} 
beiden etablierten Institutionen hätten mit einer internationalen Handelsorganisation ergänzt werden sollen (International Trade Organization ITO). Die Weltgemeinschaft konnte sich zu diesem Zeitpunkt jedoch nicht auf die Etablierung einer ITO einigen. Als Übergangslösung wurden 1947 in Genf die GATT-Verträge abgeschlossen (GATT: General Agreement on Tariffs and Trade). Das GATT war zuerst ein plurilaterales Abkommen über den Abbau von Zöllen und Handelshemmnissen in 23 Mitgliedsländern. Im Rahmen des GATT fanden acht Verhandlungsrunden statt. In jeder Runde nahm die Anzahl der Mitglieder zu. Dadurch erhielt das GATT über die Zeit den Charakter eines multilateralen Abkommens, dies in Abgrenzung zu beispielsweise bilateralen Abkommen, wie es die Schweiz mit der EU kennt oder anderen plurilateralen Abkommen wie beispielsweise das United States-Mexico-Canada Agreement (USMCA) oder das Trans-Pacific Partnership (TPP). Die letzte Verhandlungsrunde im Rahmen des GATT war die sogenannte Uruguay-Runde (1986-1994), die in Marrakesch (Marokko) beendet wurde. Drei wesentliche Ergebnisse gingen aus dieser Uruguay-Runde hervor. 1) Die WTO in ihrer bestehenden Form wurde gegründet. Dies repräsentierte eine institutionelle Reform des GATT und eine neue Eingliederung von Mitgliedsländern. 2) Das GATT bezog sich auf Handel von Gütern. Mit der Gründung der WTO wurden die Aktivitäten der Handelsorganisation auch auf Dienstleistungen und Eigentumsrechte ausgedehnt. Es entstanden neue Abkommen mit den Bezeichnungen GATS (General Agreement on Trade in Services) und TRIPS (Agreement on Trade-Related Aspects of Intellectual Property Rights). 3) In Uruguay einigten sich die Mitgliedsländer auf einen substanziellen Abbau der Agrarsubventionen im Rahmen des sogenannten "Agreement on Agriculture» (AoA). Die Schweiz war bereits am GATT beteiligt, konnte aber dank einem Sonderstatus den Agrarbereich ausklammern. Dies war im Rahmen der WTO nicht mehr möglich.

\subsubsection{Regeln und Struktur der WTO}

In der WTO sind zwei grundlegende Regeln für mehr Freihandel festgehalten (WTO, 2020b):

- Meistbegünstigung (Nichtdiskriminierung): Handelsvorteile, die ein WTOMitgliedsland einem anderen gewährt, muss es allen anderen Mitgliedsländern auch gewähren.

- Inländerbehandlung: Ausländische Waren sowie deren Anbieter dürfen nicht schlechter behandelt werden als inländische.

Zusätzlich zu diesen grundsätzlichen Handelsregeln sind die Mitglieder der WTO auch zu Transparenz und Berechenbarkeit verpflichtet. Transparenz beutet, dass Regelungen und Beschränkungen des Aussenhandels veröffentlicht werden müssen und die WTO die Handelspolitik der Mitgliedsländer überwacht. Berechenbarkeit bedeutet, dass die Länder ihre Handelsmassnahmen nicht verschärfen dürfen. Wer seine Verpflichtungen im Rahmen der WTO nicht einhält, kann sanktioniert werden. Die WTO 
figuriert dabei auch als Schlichtungsstelle für Handelskonflikte, insbesondere in Bezug auf die Umsetzung verschiedener Sicherheitsventile. So können einzelne Regeln ausgesetzt werden, wenn sich ein Land beispielsweise in Zahlungsbilanzschwierigkeiten befindet oder wenn es neue Industrien schützen will. Auch gegen Importschocks oder unfairen Handel können Massnahmen ergriffen werden, die von der WTO überwacht werden (Senti, 2000).

Wichtigstes Beschlussgremium der WTO ist die Ministerkonferenz der Mitgliedsländer, die mindestens alle zwei Jahre tagt (WTO, 2020b). Entscheidungen in Bezug auf Vertragsänderungen, die das Prinzip der Meistbegünstigung, das Inländerprinzip oder das Entscheidungsverfahren berühren, erfordern Einstimmigkeit. Für Vertragsänderungen, die die Rechte und Pflichten der Mitglieder betreffen, ist eine Zweidrittelmehrheit ausreichend. Der allgemeine Rat (General Council), zusammengesetzt aus Experten auf Beamtenebene, tagt in kürzeren Abständen. Dieses Gremium übernimmt die Organisation, Überwachung und Weiterentwicklung der einzelnen Abkommen betreffend Güter, Dienstleistungen und Eigentumsrechte. Der allgemeine Rat bestimmt die Mitglieder in Streitschlichtungsgremien (Dispute Settlement Body, DSB) und dient als Instanz zur Überprüfung der Handelspolitik der Mitgliedsländer (Trade Policy Review Mechanism, TRPM).

Der fundamentale Aspekt innerhalb der WTO ist, dass sämtliche Länder das gleiche Gewicht in der Verabschiedung der Regeln und Verträge haben und grosse Handelsnationen ihre eigenen Regeln nicht einfach durchsetzen können. Aus diesem Grund ergaben sich innerhalb der WTO-Mitgliedsländer schon früh unterschiedliche Gruppen, die versuchten, ihre gemeinsamen Handelsinteressen durchzusetzen (WTO, 2020a). Wichtige Gruppen innerhalb der WTO sind die CAIRNS-Gruppe, welche in erster Linie Länder umfasst, die über komparative Vorteile in der Nahrungsmittelproduktion verfügen und stark exportorientierte Interessen vertragen. Dazu gehören beispielsweise Australien, Kanada, Kolumbien oder Malaysia. Die USA vertraten bis zum Amtsantritt von Donald Trump 2017 eine ähnliche Politik. Als Gegengewicht zu diesen exportorientierten Volkswirtschaften (zumindest im Agrarbereich) etablierte sich die EU und die G-10. Letztere besteht aus einer Gruppe von kleinen Ländern, die Nahrungsmittel importieren und auf den Schutz ihrer inländischen Landwirtschaft pochen wie beispielsweise die Schweiz, Norwegen, Island oder Israel. Unklar ist zurzeit die Positionierung des britischen Königreichs, welches nach dem Austritt aus der EU die eigene Agrarpolitik noch definieren muss. Zwischen den exportorientierten Ländern, der EU und der G-10 positioniert sich die G-20. Diese verbindet unterschiedliche Länder von Indien über China bis Brasilien oder Argentinien. Charakteristisch für diese Länder ist, dass es sich vorwiegend um Schwellenländer handelt, die als Konzession zur Öffnung ihrer Märkte im Industrie- und Dienstleistungssektor auch Zugang zu den Agrarmärkten fordern. Eine spezielle Rolle spielen flächenmässig grosse Länder wie Russland, China oder Indien, die allein mit ihrer Grösse die Agrarmärkte 
beeinflussen. Ob eines dieser Länder zum Exporteur von Nahrungsmitteln wird oder nicht, hat eine grosse Hebelwirkung auf die dünnen Agrarmärkte weltweit. Zur Erinnerung: Dünn bedeutet in diesem Zusammenhang, dass eine relative geringe Menge der produzierten Lebensmittel überhaupt über Grenzen hinweg gehandelt werden.

Die institutionellen Regeln der WTO sind zwar stark auf die Gleichbehandlung der Mitglieder ausgerichtet. Dieser erstrebenswerte Umstand ist aber gleichzeitig auch eine grosse Schwäche der WTO. Da es eine Einigung aller Mitglieder braucht, ist ein Abschluss der Verhandlungsrunden mit dem Anstieg der Mitglieder immer schwieriger geworden. Die asymmetrischen Handelsbeziehungen zwischen Industrienationen und Entwicklungsländern konnten deshalb bis heute nie beseitigt werden. Statt innerhalb der WTO zu verhandeln, fokussierten die Länder stärker auf bilaterale und plurilaterale Abkommen (wie z.B. TTIP oder TPS). Dies schwächt die Position von multilateralen Abkommen, die aus theoretischer Sicht vorzuziehen wären, weil diese niemanden diskriminieren (Rodrik, 2018). Eine weitere Kritik an der WTO ist, dass sie keinen demokratischen Kontrollen unterworfen ist. Die WTO ist nicht den Vereinten Nationen (UN) unterstellt und auch undemokratische Staaten sind WTO-Mitglieder. Die Verhandlungen und auch die Rechtsprechung erfolgt auf der Stufe der Minister bzw. Expertenpanels. Schliesslich sind auch viele sogenannte «Non-Trade Concerns» wie beispielsweise Menschenrechte oder Sozialstandards nicht direkt Teil der Verhandlungen. Eine Ausnahme hierbei ist aber gerade die Landwirtschaft. Aspekte wie "Food Security» (Ernährungssicherheit) und Agrarumweltmassnahmen werden im Rahmen des sogenannten "Agreements on Agriculture» (AoA) behandelt (Wohlmeyer und Quendler, 2017).

\subsubsection{Agreement on Agriculture}

Das AoA war das Kernstück der Verhandlungen im Rahmen der Uruguay-Runde (Senti, 2000). Es kannte drei Verhandlungsbereiche: Marktzutritt, inländische Stützung und Exportsubventionen. Unter Marktzutritt werden sämtliche Aspekte des Grenzschutzes wie beispielsweise Zölle oder Zollkontingente zusammengefasst. Diese Instrumente wirken sich auf die landwirtschaftlichen Märkte aus und verzerren direkt die Preise für Agrarprodukte. Zum Bereich inländische Stützung gehören Zulagen, Direktzahlungen oder Inputverbilligungen, die sich nicht direkt auf die Märkte für landwirtschaftliche Produkte auswirken und nur indirekt die Preise beeinflussen. Exportsubventionen beeinflussen ebenfalls direkt die Preise auf den Märkten. Theoretische und empirische Evidenz zeigen, dass Exportsubventionen die Wohlfahrt sowohl im In- als auch im Ausland verschlechtern (siehe Kapitel 8.2). Mit der Gründung der WTO herrschte daher Einigkeit, dass Exportsubventionen abgeschafft werden sollten. Sie bildeten daher von Anfang an einen eigenen Bereich. Allerdings sind bis heute noch nicht alle Exportsubventionen eliminiert. Die Schweiz beispielsweise hat erst im Jahr 2019, das sogenannte "Schoggigesetz», eine Inputverbilligung für Milch 
und Weizen, wenn diese in Form von Schokolade exportiert werden, aufgehoben bzw. in ein neues Subventionssystem überführt.

Für die Regelung des Marktzutritts war es elementar, dass sich die Länder im Rahmen der Verhandlungen auf eine Zollreduktion einigten. Dafür wurden sogenannte «nicht tarifäre Handelshemmnisse", wie beispielsweise Importquoten oder Diskriminierungen in der Zollabwicklung, im Zuge des AoA in Zolläquivalente umgewandelt. Man spricht in diesem Zusammenhang von Tarifierung (Tariffication). Für alle Mitgliedsländer wurde ein Basiswert für die Periode 1986-1988 berechnet, der als Ausgangspunkt für die Verhandlung von Zollreduktionen diente. Industrienationen mussten Zölle um $36 \%$ reduzieren (mindestens um $15 \%$ in jeder Tariflinie). Die Reduktion für Entwicklungsländer lag bei 24 bzw. $10 \%$. Die ärmsten Länder (Least-Developed Countries, LDC) mussten ihre Zölle nicht reduzieren. Ausserdem wurden im Rahmen von «Special Treatments» Ausnahmen für besonders sensitive Produkte gewährt. Im Falle von z.B. Importschocks oder tiefen Importpreisen dürfen Schutzmechanismen, die den Marktzutritt erschweren, erlassen werden. Kritiker dieser Tarifierung argumentieren, dass durch das Festsetzen von Basiswerten Zölle erst recht legitimiert worden seien. Aus einer puritanischen Marktsicht wäre es vorzuziehen gewesen, sämtliche Marktbarrieren abzuschaffen. Es muss allerdings festgehalten werden, dass gemäss aktuellen Zahlen viele Länder mittlerweile viel tiefere Schutzzölle haben, als sie gemäss WTO-Vereinbarung haben dürften (Bureau et al., 2019). Es sind in erster Linie bilaterale Abkommen, welche zu einer Liberalisierung des Agrarmarktes führen und weniger die multilateralen Abkommen im Rahmen der WTO. Trotzdem bleibt die WTO gerade auch für Entwicklungsländer ein wichtiger Akteur, wenn es um die Entwicklung der Wirtschaft geht (Desta, 2016).

Im Bereich der inländischen Stützung werden drei Kategorien von Massnahmen unterschieden. Diese werden jeweils in sogenannten Boxen zusammengefasst. Die Green Box umfasst Massnahmen, die im Rahmen der WTO erlaubt sind. Es handelt sich vorwiegend um Massnahmen, die für den Erhalt von öffentlichen Gütern gesprochen werden und keinen oder nur einen sehr geringen Einfluss auf die Agrarmärkte haben. Das heisst, sie sollten möglichst unabhängig von der landwirtschaftlichen Produktionsentscheidung sein. Sie sind dadurch nicht oder nur minimal handelsverzerrend. Ein klassisches Beispiel für diese Art von Massnahmen sind Zahlungen für die Förderung von Biodiversität. In der Blue Box sind Massnahmen zusammengefasst, die zwar einen Einfluss auf die landwirtschaftliche Produktion haben, gleichzeitig aber an Produktionslimiten geknüpft sind. Ein Beispiel für diese Art von Massnahmen sind Beiträge an extensive Produktionssysteme (Bio) oder Flächenbeiträge, die an gewisse Umweltbedingungen geknüpft sind (Cross-Compliance). Die Amber Box schliesslich umfasst diejenigen Stützungen, die nur unter strikten Vorschriften erlaubt sind und eigentlich abgebaut werden sollten. Diese erhöhen normalerweise 
direkt die landwirtschaftliche Produktion. Dazu gehören beispielsweise Inputverbilligungen wie die Verkäsungszulage, welche eine handelsverzerrende Massnahme darstellt.

Grundsätzlich ist das System der Boxen in der WTO so aufgebaut, dass die zu beurteilenden Massnahmen auf einer ordinalen Skala eingeordnet werden, wie stark sie die Produktion beeinflussen können. Massnahmen, welche die Produktion oder den Produktpreis direkt beeinflussen, gehören in die Amber Box. Aus Sicht des AoA sollten diese abgebaut werden. Wirkt sich die Massnahme zwar auf die Produktion aus, wird sie aber gleichzeitig eingeschränkt, dann gehört sie in die Blue Box. Einschränkungen, die sich auf die aktuelle Produktion beziehen, sind dabei schlechter als solche, die sich auf die Produktion in der Vergangenheit beziehen. Ebenfalls in die Blue Box gehören Flächenzahlungen, die an Umweltbedingungen geknüpft sind. Grünes Licht erhalten diejenigen Massnahmen, welche die Produktion der Landwirte kaum oder nicht beeinflussen. Entlang dieser Kategorien wird auch in den laufenden Verhandlungen der WTO über Massnahmen im Bereich der inländischen Stützung diskutiert.

\subsubsection{Entwicklung der WTO seit der Uruguay-Runde}

Nach Abschluss der Uruguay-Runde wurde die Doha-Runde gestartet. Diese bezeichnet ein Paket von Themen, das die Wirtschafts- und Handelsminister der WTO-Mitgliedstaaten 2001 auf ihrer Konferenz in Doha verhandelten. Diese Verhandlungen hätten eigentlich schon bis 2005 abgeschlossen werden sollen. In Bezug auf die Landwirtschaft enthält die Doha-Runde folgende Kernelemente:

- Verbesserter Marktzugang für alle Mitgliedsländer (d.h. eine Reduktion der Zölle um $70 \%)$;

- Abschaffung sämtlicher Exportsubventionen;

- Reduktion der handelsverzerrenden heimischen Stützung innerhalb der Amber Box;

- $\quad$ Einbezug von Nicht-Handelsanliegen wie Ernährungssicherheit oder ländliche Entwicklung («Non-Trade Concerns»);

- Behandlung spezifischer Probleme von Entwicklungsländern (daher manchmal auch «Doha-Entwicklungsrunde» genannt).

Die Verhandlungen innerhalb der Doha-Runde verlaufen zäh. Erste Resultate konnten erst 2013, d.h. zwölf Jahre nach dem Start der Verhandlungen, erzielt werden. Die Umsetzung der beschlossenen Massnahmen erfolgte erstmals 2017. Insbesondere im Bereich der Agrarprodukte blieb die Verhandlungsrunde aber weit hinter den gesteckten Zielen zurück und das Ziel des vollständigen Freihandels wurde aufgegeben (Scott, 2017). Aus diesem Grund entstanden in den letzten Jahren immer mehr plurilaterale 
und bilaterale Abkommen, die als Ventil für die zähen Verhandlungen in der WTO dienten (Bureau et al., 2019). Nach der Amtszeit von Präsident Trump in den USA bleibt das Schicksal der Doha-Runde weiterhin offen und ein Abbruch der Verhandlungen und ein Neustart unter anderen Zielvorgaben wird oft als einziger Ausweg gesehen. Insbesondere eine Abkehr von der Einstimmigkeit wird als möglicher Ausweg aus dem Stillstand der Verhandlungen gesehen. Aber auch eine breitere Abstützung z.B. mit Blick auf die Nachhaltigkeitsziele der UNO werden diskutiert (WTO, 2020c).

\subsection{OECD (Organization for Economic Cooperation and Develop- ment $)^{5}$}

Die OECD vereint 36 Mitgliedsländer, die sich im Kern zu Demokratie und Marktwirtschaft bekennen. Der Zweck der Organisation ist es, die Mitgliedsländer zu beraten. Dabei sollen Erfahrungen über Politikansätze diskutiert und Lösungen für gemeinsame Probleme gefunden werden. Darüber hinaus können erfolgreiche Politikstrategien ausgetauscht und nationale und internationale Politik koordiniert werden. Die Aufgabe der OECD ist in erster Linie die Förderung von Wirtschaftswachstum und Beschäftigung sowie die Erhöhung des Lebensstandards in den Mitgliedsländern. Dazu gehören auch die Förderung stabiler öffentlicher Finanzen, die Unterstützung der wirtschaftlichen Entwicklung von Drittstaaten und die Ausweitung des Welthandels.

Um diesen Zweck zu erfüllen, liefert die Organisation Statistiken und Indikatoren zu Wirtschaft und Gesellschaft. Sie analysiert und prognostiziert makroökonomische Entwicklungen und untersucht den gesellschaftlichen Wandel in vielfältigen Bereichen, z.B. Handel, Umwelt, Landwirtschaft, Technologie oder Steuern. Die Kernthemen der OECD sind Beschäftigung, Bildung, Sozialpolitik, Wirtschaft, Finanzen, Governance, Umwelt, nachhaltige Entwicklung und Innovation. Die OECD baut auf den Prinzipien der Zusammenarbeit, des Dialogs und des Konsenses auf. Mit ihren Gutachten und Berichten trägt die OECD aktiv zu den agrarpolitischen Diskussionen in der Schweiz bei.

\subsubsection{Aufbau der OECD}

Die OECD besteht aus drei Bereichen: Rat, Sekretariat und Ausschüsse. Der Rat setzt sich zusammen aus Vertretern der Mitgliedsländer und fällt seine Entscheidungen im Konsens. Er ist für die strategische Führung und für die Aufsicht über die OECD verantwortlich. Das Sekretariat erarbeitet Analysen und macht Vorschläge für die Ver-

\footnotetext{
${ }^{5}$ Diese Ausführungen basieren vorwiegend auf den Informationen der OECD-Webseite: http://www.oecd.org/.
} 
besserung von Politiken. In den Ausschüssen werden spezifische Themen wie Steuern oder auch die Landwirtschaftspolitik in einem gemischten Gremium (auch NichtMitgliedsländer) diskutiert und analysiert.

\subsubsection{Producer Support Estimate, PSE}

Ein zentraler Indikator für die Agrarpolitik ist das Erzeugerstützungsmass PSE (Producer Support Estimate). Dieser Indikator wird für den internationalen Vergleich von Agrarpolitiken verwendet (OECD, 2020). Das PSE ist ein breit anerkannter und viel verwendeter Indikator. Unter dem Begriff Erzeugerstützungsmass (oder schlicht dem Support) für die Landwirtschaft wird der Transfer von Konsumenten und Steuerzahlern hin zu den landwirtschaftlichen Produzenten verstanden, der sich aus den agrarpolitischen Massnahmen ergibt. Das bedeutet, dass der Indikator neben den Budgetausgaben des Bundes für die Landwirtschaft auch sämtliche andere Transfers, z.B. Zölle oder Kreditverbilligungen, messen soll.

Das Mass zeigt daher die effektive Stützung, welche die Agrarpolitik den Landwirten zukommen lässt aus einer ganzheitlichen Perspektive. Dabei ist der Indikator modular aufgebaut, sodass unterschiedliche Regulierungsebenen, z.B. Marktzutritt und inländische Stützung, separat abgebildet werden können (Producer Support Estimate, Market Price Support, Consumer Support Estimate, General Services Support Estimate, Total Support Estimate). Das PSE misst aber weder den Effekt der Politikmassnahmen auf die Produktion noch das Einkommen der Bauern oder die Umwelt. Im internationalen Kontext gibt es dazu eine neue Organisation (International Organisations Consortium for Measuring the Policy Environment for Agriculture), welche diese Vergleichsmöglichkeiten ausserhalb der OECD ermöglichen soll.

Analog zu den Boxen in der WTO werden auch in der OECD die unterschiedlichen Instrumente der Agrarpolitik eingeordnet. In Analogie zur WTO werden die Transfers mit Blick auf ihre Produktionswirkung gegliedert (siehe auch Box 1):

- $\quad$ Support basierend auf Produktion (z.B. Zölle).

- Zahlungen, die auf der Nutzung von Inputs beruhen (z.B. Rückerstattung der Mineralölsteuer).

- Zahlungen basierend auf aktueller Anzahl Tiere oder genutzter Flächen Produktion des entsprechenden Produkts ist obligatorisch (z.B. Zahlungen für Zuckerrübenanbau).

- Zahlungen basierend auf nicht aktuellen Zahlen (historische Zahlungen) mit obligatorischer Produktion (z.B. Versorgungssicherheitsbeiträge).

- Zahlungen basierend auf historischen Zahlen, Produktionsentscheid dem Landwirt überlassen (z.B. allgemeine Direktzahlungen bis 2014).

- Zahlungen basierend auf Nicht-Produktionsleistungen (z.B. Beitrag für Biodiversitätsförderflächen). 
- Verschiedene Zahlungen (z.B. Forschung) und administrative Kosten (General Service Support).

Im Vergleich mit anderen Ländern hat die Schweiz eine sehr hohe Stützung des Agrarsektors (OECD, 2015). Ende der 1980er-Jahre lag der Wert bei knapp $80 \%$. In anderen Worten, von jedem Franken, den die Schweizer Bauern verdienten, stammten 80 Rappen direkt oder indirekt vom Staat. Seit der neuen Agrarpolitik, die in den 1990er-Jahren startete, sank dieser Anteil und liegt jetzt bei ca. 50 \%. Das bedeutet, dass nach wie vor mehr als die Hälfte des Einkommens der Bauern aus agrarpolitischen Massnahmen stammt.

Trotz dieser starken Unterstützung illustriert das PSE eindrücklich die Agrarreform, welche die Schweizer Landwirtschaftspolitik in den letzten 20 Jahren prägte. Die Trennung der Preis- und Einkommenspolitik und die damit einhergehende Einführung der Direktzahlungen führte zu einer Verschiebung der Art der Unterstützung (siehe auch Kapitel 7.3).

Der grösste Anteil der Direktzahlungen unterstützt die landwirtschaftliche Produktion nicht direkt. Die allgemeinen Direktzahlungen, welche bis 2014 das zentrale Element der inländischen Stützung war, gehörten zu den Zahlungen, die auf historischen Zahlungen beruhten, und überliessen den Produktionsentscheid dem Landwirt. Im Gegenzug wurden Preis-Absatz Garantien und andere produktionsstützende Massnahmen aufgehoben. Dies führte zu einer massiven Verschiebung der Stützung in Bereiche, welche sowohl die OECD als auch die WTO als weniger problematisch ansehen. Aus Sicht der OECD führte die Einführung der Versorgungssicherheitsbeiträge, die eine obligatorische Produktion erfordern (über den Mindestbesatz), zu einem Rückschritt, weil dieses Instrument die Produktion wieder stärker fördert als die allgemeinen Direktzahlungen.

Obwohl das PSE als Indikator breit abgestützt ist, gibt es auch Kritik an der monetarisierten Vermessung der Agrarpolitik. Aus Sicht der Schweizer Agrarpolitik ist anzuführen, dass der Indikator nichts über das Kostenumfeld, die Umweltwirkung und die Kaufkraft in den verschiedenen Ländern aussagt. Es bleibt daher schwierig abzuschätzen, wie stark das landwirtschaftliche Einkommen durch agrarpolitische Eingriffe gestützt wird und wie viel diese Unterstützung (kaufkraftbereinigt) dem Landwirt wert ist. Auch unterscheidet das PSE keine (Prozess-)Qualitäten beispielsweise im Tierbereich. Es wird lediglich der Preisunterschied zwischen dem Weltmarkt und dem inländischen Produzentenpreis als Marktstützung definiert. Gerade dort, wo die Tierschutzvorschriften weltweit unterschiedlich sind, z.B. im Bereich der Geflügelmast, kann dies zu einer Verzerrung des Stützungsmasses führen. 
Einführung in die Schweizer Agrarpolitik

Box 1 Entwicklung des Producer Support Estimate PSE in der Schweiz

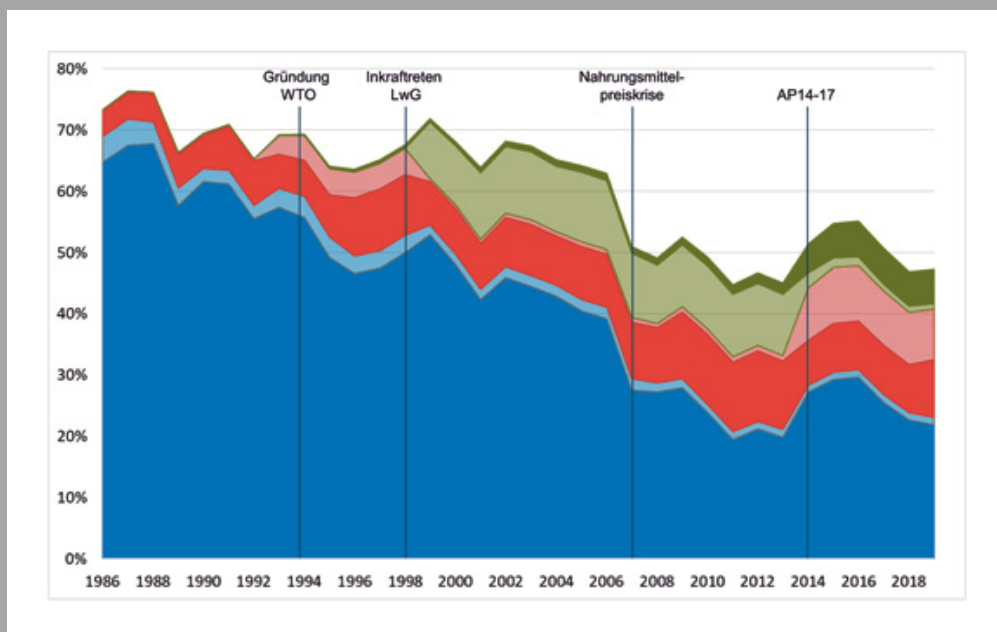

Die Zahlungen basierend auf direkter Unterstützung der Produktion (z.B. Grenzschutz) sind in der Entwicklung des PSE blau eingezeichnet. Rot sind diejenigen Stützungsmassnahmen, welche sich auf die aktuelle Anzahl der Tiere oder Flächen beziehen. Die Unterstützung, welche sich auf Umwelt- oder öffentliche Güter bezieht, ist in Grün eingezeichnet.

Die Übersicht zeigt, dass im Zeitverlauf die Höhe des PSE zurückgeht von ca. 80 \% Ende der 1980er-Jahre auf knapp 50 \% im Jahr 2018. Gleichzeitig hat sich auch die Zusammensetzung des PSE stark verändert. Ursprünglich war die Unterstützung der Schweizer Landwirtschaft ausschliesslich auf den Grenzschutz fokussiert. Mit der Gründung der WTO und dem neuen Verfassungsartikel 104 bzw. mit der Einführung des darauf basierenden Landwirtschaftsgesetzes von 1999 wurde der Grenzschutz sukzessive abgebaut und durch andere Stützungsmassnahmen ersetzt. Ein wichtiger Anteil waren die Flächenbeiträge, welche keine Produktionsvorgaben enthielten. In der AP14-17 wurden die Flächenbeiträge durch die Versorgungssicherheitsbeiträge ersetzt. Letztere bedingen einen Mindesttierbesatz auf den entsprechenden Flächen. Deshalb dehnte sich der Anteil der roten Stützungsmassnahmen in den letzten Jahren wieder aus. Das Absinken des PSE ist aber nicht in jedem Fall mit einer Anpassung der inländischen Agrarpolitik verbunden. Mit höheren Preisen in der Nahrungsmittelkrise 2008 sank beispielsweise das Niveau des PSE, ohne dass die Schweiz grosse Veränderungen an der Agrarpolitik vorgenommen hätte. 
Dazu gehört auch, dass positive Externalitäten explizit ausgeklammert werden und die Unterscheidung in Einkommenstransfers und Abgeltung von öffentlichen Gütern nur schwer nachzuvollziehen ist. Schliesslich ist die Änderung des Grenzschutzes manchmal gar nicht auf agrarpolitische Massnahmen zurückzuführen, sondern auf exogene Einflüsse wie beispielsweise Preisentwicklung auf den internationalen Agrarmärkten und/oder Kurs- und Währungsschwankungen. Eine Erhöhung der Weltmarktpreise führt zu einer Reduktion des PSE in der Schweiz, auch wenn das Grenzschutzregime gleich bleibt. So ist der errechnete Grenzschutz für die Schweiz seit 2013 vor allem aufgrund des Erstarkens des Schweizer Frankens infolge der Nationalbankentscheide substanziell angestiegen und nicht etwa, weil die Schweiz protektionistischer geworden wäre.

\subsection{Food and Agriculture Organization of the United Nations, $\mathrm{FAO}^{6}$}

Die Ernährungs- und Landwirtschaftsorganisation der Vereinten Nationen spielt eine zentrale Rolle bei der weltweiten Koordination, der technischen Unterstützung und der Vernetzung der Forschung im Bereich der Ernährungssicherheit. Sie unterstützt die Sicherung der Existenzgrundlagen der ländlichen Bevölkerung und der nachhaltigen Nutzung und Verwaltung von natürlichen Ressourcen. Die Ziele der FAO sind die Bekämpfung von Hunger, die Förderung von Ernährungssicherheit und die Reduktion von Mangelernährung. Ausserdem setzt sich die Organisation für die Beseitigung von Armut sowie für eine Förderung des sozialen und wirtschaftlichen Fortschritts für alle ein. Die FAO fokussiert auf ein nachhaltiges Management und die Nutzung von natürlichen Ressourcen wie Boden, Wasser, Luft, Klima und genetischen Ressourcen zugunsten heutiger und künftiger Generationen.

Die FAO hat ein breites Tätigkeitsfeld. Sie fungiert als Wissensnetzwerk und publiziert Newsletter, Berichte Bücher, Magazine und Daten (FAOSTAT). Darüber hinaus berät sie die Mitgliedstaaten bei der Agrar- und Entwicklungspolitik und bietet eine Plattform für den internationalen Austausch über Agrar- und Ernährungsthemen. Die FAO betreut Projekte in der ganzen Welt, bietet technisches Wissen an und garantiert finanzielle Unterstützung. In Krisenfällen arbeitet sie mit dem Welternährungsprogramm (WFP, World Food Programme) und anderen humanitären Organisationen zusammen.

Im Jahr 2021 fand der «Food Systems Summit» statt. Dieser sogenannte Welternährungsgipfel soll einen wichtigen Beitrag zur Erreichung der nachhaltigen Entwicklungsziele (SDG) leisten, indem die Notwendigkeit für die Anpassung der Landwirtschafts- und Ernährungssysteme Eingang in den öffentlichen und politischen Diskurs

\footnotetext{
${ }^{6}$ Weitere Informationen zur FAO: http://www.fao.org/.
} 
findet. Das Ziel ist es, nicht nur die Mitgliedsländer für die Herausforderungen zu sensibilisieren, sondern auch konkrete gesellschaftliche und politische Lösungsansätze aufzuzeigen die, wie z.B. das Pariser Klimaabkommen, mittel- und langfristig zu verbindlichen Veränderungsprozessen führen sollen. Auf dem Summit haben über 80 Länder eine Erklärung abgegeben, wie sie eine Transformation zu einem nachhaltigen Landwirtschafts- und Ernährungssystem anstreben möchten.

Die Schweiz ist seit 1946 Mitglied der FAO. Das Bundesamt für Landwirtschaft (BLW) ist verantwortlich für die Vertretung der Interessen der Schweiz. Es wird u.a. beratend unterstützt vom schweizerischen nationalen FAO-Komitee (CNS-FAO), einem Konsultativorgan des Bundesrats für Fragen zur Ernährungssicherheit und nachhaltigen Ernährungssystemen. Die Schweiz ist Mitglied in mehreren Ausschüssen der FAO wie beispielsweise Committee on World Food Security, Committee on Agriculture, Committee on Forestry und Committee on Commodity Problems sowie in der Commission on Genetic Resources for Food and Agriculture. Das Arbeitsprogramm der FAO wird über obligatorische und freiwillige Beiträge finanziert. Ihr Budget für 20182019 betrug 2,6 Mia. US-Dollar und setzte sich zu 39 \% aus Pflichtbeiträgen der Mitgliedstaaten und zu $61 \%$ aus freiwilligen Beiträgen der Mitgliedstaaten und anderer Partner zusammen. Die Schweiz setzt sich insbesondere für Aspekte der Agrarökologie, einem Kernanliegen der FAO, ein.

\subsection{Weitere Institutionen im Kontext der internationalen Agrar- politik}

Ausser der WTO, der OECD und der FAO existieren noch weitere internationale Organisationen, welche direkt oder indirekt einen Einfluss auf die Agrarpolitik weltweit haben. Dazu gehören Organisationen, die landwirtschaftliche Projekte finanzieren, z.B. die Weltbank oder der Fonds für landwirtschaftliche Entwicklung der UN, Forschung betreiben, wie CGIAR oder IAASTD, aber auch übergeordnete Wissensgemeinschaften wie der IPCC, das IPBES oder das Millenium Ecosystem Assessment. Schliesslich bildet auch die UNO-Agenda 2030 einen wichtigen Eckpfeiler für die Entwicklung der Agrarpolitik in vielen Ländern weltweit.

- $\quad$ IFAD (International Fund for Agricultural Development)

- Weltbank (Agrarforschung und Projekte)

- $\quad$ CGIAR (Consultative Group on International Agricultural Research)

- IAASTD (International Assessment of Agricultural Knowledge, Science and Technology for Development): Weltagrarbericht 2009

- $\quad$ IPCC (Intergovernmental Panel on Climate Change)

- IPBES: The Intergovernmental Science-Policy Platform on Biodiversity and Ecosystem Services 
- $\quad$ Millenium Ecosystem Assessment 2005: Ecosystem Services (MEA, 2005)

- UNO-Agenda 2030 (u.a. SDG 2: Den Hunger beenden, Ernährungssicherheit und eine bessere Ernährung erreichen und eine nachhaltige Landwirtschaft fördern \& SDG 12: Für nachhaltige Konsum- und Produktionsmuster sorgen)

\section{ZUSAMMENFASSUNG}

Wichtige internationale Akteure in der Agrarpolitik sind die WTO, die OECD und die FAO. Die WTO war ursprünglich die treibende Kraft in der Agrarmarktliberalisierung. Sie definiert die Verhandlungsbereiche Marktzutritt, inländische Stützung und Exportsubventionen und kategorisiert die agrarpolitischen Massnahmen in unterschiedliche Boxen (Green, Blue, Amber). Innerhalb dieser Boxen soll der Marktzutritt erhöht und die Stützung reduziert werden. Die WTO steht für multilaterale Abkommen mit klaren Regeln. Die Verhandlungen in der WTO sind zäh und werden laufend von plurilateralen oder bilateralen Abkommen torpediert. Die laufende Verhandlungsrunde (Doha) steht daher vor einem ungewissen Ausgang und eine Reform der WTO wird diskutiert. Die OECD ist ein wichtiger Player in der Definition von Standards für die Agrarpolitik. Diese Standards, insbesondere das Producer Support Estimate (PSE), ermöglichen den Vergleich von Agrarpolitiken mit unterschiedlichen Massnahmen über Länder hinweg. Das Ziel der OECD sind marktpolitisch motivierte Empfehlungen für die Agrarpolitik in den Mitgliedsländern. Sie hat einen forschungsorientierten Ansatz (science-based policy making). Die FAO wiederum engagiert sich in erster Linie für Entwicklungsländer. Sie vermittelt agronomisches Know-how in diversen Projekten in der ganzen Welt und ist gleichzeitig die Informationsplattform für diverse Organisationen im Kontext der Agrarpolitik.

\section{WEITERFÜHRENDE LITERATUR}

Die Webseiten der drei Organisationen (WTO, OECD und FAO) sind eine beinahe unerschöpfliche Quelle für Informationen im Kontext der Landwirtschaft. Dazu gehören nicht nur spezifische Publikationen und Daten, sondern auch viele aufgearbeitete Informationen in Factsheets, interaktiven Grafiken oder Filmen.

WTO

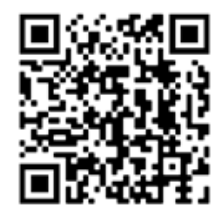

$O E C D$

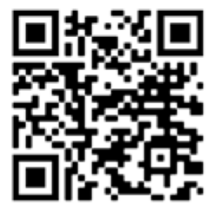

FAO

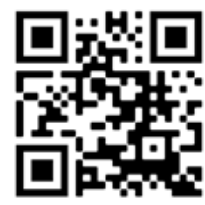


Einführung in die Schweizer Agrarpolitik

Im internationalen Kontext sind der etwas ältere Bericht zum Zustand der Ökosysteme "The Millennium Ecosystem Assessment» und der Weltagrarbericht "Agriculture at a crossroads - global report» vom Gremium mit dem Namen International Assessment of Agricultural Knowledge, Science and Technology for Development wichtige Meilensteine. Die eigentliche Quelle ist aber erst im Entstehen begriffen: Aus dem World Food Summit 2021 soll eine Art Roadmap für die Entwicklung der Landwirtschaft weltweit hervorgehen mit verbindlichen Zielen und Instrumenten. 


\section{Konzeptionelle Grundlagen der Schweizer Agrarpolitik}

\section{READERS' GUIDE}

In diesem Kapitel werden konzeptionelle Grundlagen der Schweizer Agrarpolitik vorgestellt. Diese sind wichtig, um den Aufbau, die Entwicklung und die Beurteilung des agrarpolitischen Instrumentariums zu verstehen. Die konzeptionellen Grundlagen sind in fünf Kapitel unterteilt: 1) Ziele und Zielkonflikte; 2) Grundlagen der Agrarmarktordnung; 3) Multifunktionalität und Entkopplung; 4) Optimale Agrarumweltpolitik sowie 5) Beurteilungskriterien für die Agrarpolitik. Die einzelnen Kapitel stehen nur in einer losen Verbindung zueinander. Sie führen daher mehr oder weniger unabhängig voneinander unterschiedliche Begrifflichkeiten und Definitionen ein. Das Ziel dieses Kapitels ist es dann auch, dass die Leserinnen und Leser diese wichtigen Begriffe kennenlernen und deren Bedeutung für die Schweizer Agrarpolitik einordnen können.

\subsection{Ziele und Zielkonflikte in der Agrarpolitik}

\subsubsection{Zielsystem}

Eine Grundlage für das Verständnis der Agrarpolitik ist die Kenntnis über das Zielsystem, welches den Massnahmen und Regelungen zugrunde liegt.

In den demokratisch und marktwirtschaftlich orientierten Gesellschaften gibt es einen gewissen Konsens über die gesellschaftspolitischen Grundziele. Dazu gehören Freiheit (formal und materiell); Gerechtigkeit, Sicherheit (sozialer Frieden, wirtschaftliche Sicherheit) und Wohlstand (materielle und immaterielle Güter). Selbstverständlich sind diese Grundprinzipien lediglich als Leitplanken für die Ausgestaltung der gesellschaftlichen Ordnung gedacht. Auch diese Grundziele können unterschiedlich interpretiert werden und unterliegen damit einem Werturteilsproblem. Gerechtigkeit, beispielsweise, kann als Chancengleichheit interpretiert werden (es ist gerecht, wenn alle die gleichen Chancen haben; «liberale» Sichtweise) oder als Bedarfsgerechtigkeit (es ist gerecht, wenn alle, die eine Leistung erbringen, entsprechend entschädigt werden; «sozialistische» Perspektive). 
Für die Agrarpolitik braucht es daher eine Konkretisierung dieser übergeordneten gesellschaftlichen Ziele. Dieser Schritt ist nicht trivial und entsprechend gibt es unterschiedliche Kategorisierungen der agrarpolitischen Ziele. Für die Agrarwirtschaftspolitik, die den marktwirtschaftlichen Systemen in den westeuropäischen Ländern zugrunde liegt, sind dies folgende Ziele:

- Effizienzziele: Effizienter Einsatz der volkswirtschaftlichen Produktionsfaktoren unter Berücksichtigung von positiven und negativen Externalitäten;

- Verteilungsziele: Gerechte Einkommens- und Vermögensverteilung;

- Sicherungsziele: Stabilisierung des Wirtschaftskreislaufs und der Versorgungssicherheit;

- Wirtschaftliche Freiheit.

Dieses marktwirtschaftlich orientierte Zielsystem wurde in der Geschichte der Agrarpolitik immer wieder erweitert oder ergänzt. Neben den ökonomischen Zielen verfolgte die Agrarpolitik schon relativ früh auch soziale Ziele. Im Verlaufe der letzten Jahrzehnte wurden ökologische Ziele immer wichtiger. Die Agrarpolitik wurde dadurch zu einer «Nachhaltigkeitspolitik». Das heisst, die Regelungen des Sektors fokussierten auf die simultane Umsetzung von ökonomischen, ökologischen und sozialen Zielen, um die Produktionsgrundlagen auch für die kommenden Generationen sicherzustellen.

Wie der vorangegangene Abschnitt impliziert, sind Zielsysteme in der Agrarpolitik nicht statisch. Sie passen sich den gesellschaftlichen und wirtschaftlichen Entwicklungen an. Die Entwicklung des Zielsystems in der Schweiz illustriert diesen Aspekt gut (Rieder und Anwander Phan-huy, 1994). Die ersten agrarpolitischen Massnahmen dienten der Erhaltung der landwirtschaftlichen Produktion (Produktionsziele) und der Sicherstellung eines inländischen Angebots (Sicherungsziele). Schon bald verfolgten die agrarpolitischen Instrumente aber auch soziale Ziele wie die Reduktion der Verschuldung und der Verarmung der Landwirte. Auch der Ausgleich zwischen Landwirten im Mittelland und den Bergregionen wurde zu einem wichtigen Anliegen der Agrarpolitik. Im Landwirtschaftsgesetz von 1951, das auf dem neuen Wirtschaftsartikel in der Verfassung von 1947 beruhte, kam dieser Fokus auf Produktions-, Sicherungs- und soziale Ziele klar zum Ausdruck. Die Kernziele waren: 1) Produktion von und Versorgung mit gesunden Nahrungsmitteln, 2) Erhaltung eines gesunden Bauernstandes, 3) Leistungsfähige Landwirtschaft und 4) Schutz wirtschaftlich benachteiligter Gebiete. Diese Ziele prägten die Entwicklung der Agrarpolitik über einen langen Zeitraum, bevor innere und äussere Kräfte eine Anpassung erwirkten (siehe dazu auch das Kapitel 7) und damit die neue Verfassungsgrundlage ermöglichten. Die Massnahmen in diesem Zielsystem waren hauptsächlich auf den Grenzschutz und den Absatz der inländischen Produkte fokussiert. 
Das Zielsystem der Schweizer Agrarpolitik erfuhr eine massgebliche Neuausrichtung basierend auf dem grundlegenden Anspruch, die Preis- von der Einkommenspolitik zu entkoppeln. Der neue Verfassungsartikel Art. 104 BV von 1996 führte die Multifunktionalität der Landwirtschaft als Leitidee für die Agrarpolitik ein (Huber, 2010). Die Multifunktionalität beschreibt, dass die Landwirtschaft im Kern nach wie vor nachhaltig auf den Markt ausgerichtete Nahrungsmittel produziert. Gleichzeitig soll sie aber einen Beitrag leisten zur
a. $\quad$ sicheren Versorgung der Bevölkerung (Food Security);
b. Erhaltung der natürlichen Lebensgrundlagen und zur Pflege der Kulturland- schaft;
c. dezentralen Besiedlung des Landes.

Auf dieser Basis soll der Bund das bäuerliche Einkommen durch Direktzahlungen zur Erzielung eines angemessenen Entgelts für die erbrachten Leistungen ergänzen. Voraussetzung für den Erhalt der Direktzahlung ist ein Leistungsnachweis, der sicherstellt, dass die Landwirtschaft ökologische Minimalanforderungen einhält (ökologischer Leistungsnachweis, ÖLN). Darüber hinaus soll der Bund Produktionsformen fördern, die besonders naturnah, umwelt- und tierfreundlich sind und die Umwelt vor Beeinträchtigungen durch überhöhten Einsatz von Düngern, Chemikalien und anderen Hilfsstoffen schützen. Die Verfassungsgrundlage von 1996 hielt aber auch explizit fest, dass der Bund nur bodenbewirtschaftende bäuerliche Betriebe fördert. Dadurch wurden nicht nur ökologische, sondern auch soziale Anforderungen an eine multifunktionale Landwirtschaft in der Verfassung festgehalten - insbesondere durch die Verankerung der Festigung des bäuerlichen Grundbesitzes in der Verfassung, welche als Grundlage für das Bundesgesetz über das bäuerliche Bodenrecht (BGBB) dient. Schliesslich sieht der Verfassungsartikel vor, dass der Bund Vorschriften zur Deklaration von Herkunft, Qualität, Produktionsmethode und Verarbeitungsverfahren für Lebensmittel erlässt. Damit sind die Sicherungsziele in Bezug auf die Nahrungsmittelsicherheit (Food Safety) explizit Teil der Verfassungsgrundlage von 1997. Dieser Artikel 104 der Bundesverfassung wurde 2017 um einen weiteren Artikel ergänzt (Huber und Finger, 2019). Der Bund soll folgende Voraussetzungen für die Versorgung der Bevölkerung mit Lebensmitteln (Ernährungssicherheit) schaffen und Grundlagen für die landwirtschaftliche Produktion, insbesondere das Kulturland sichern: eine standortangepasste und ressourceneffiziente Lebensmittelproduktion sicherstellen; eine auf den Markt ausgerichtete Land- und Ernährungswirtschaft erhalten; grenzüberschreitende Handelsbeziehungen sichern, zur nachhaltigen Entwicklung der Land- und Ernährungswirtschaft beitragen und einen ressourcenschonenden Umgang mit Lebensmitteln sicherstellen (Verhinderung von Food Waste). 
Die Entwicklung der agrarpolitischen Ziele illustriert zwei Aspekte. Einerseits hat sich die Palette der Ziele erweitert. Insbesondere Umweltziele wie Landschaftspflege oder Erhaltung der Ressourcen fanden erst mit dem Verfassungsartikel von 1996 explizit Eingang in die Agrarpolitik. Mit der Ergänzung von 2017 erscheinen erstmals auch Aspekte des Food Waste in der Verfassung. Andererseits unterliegen die Ziele auch immer einer Gewichtung. Nach den Weltkriegen standen die Produktions- und Sicherungsziele im Vordergrund. Durch die Trennung der Preis- von der Einkommenspolitik verloren diese Ziele etwas an Bedeutung. Die Ergänzung durch den Absatz zur Ernährungssicherheit hingegen sollte die Produktions- und Sicherungsziele in der Agrarpolitik wieder stärker gewichten. Die genaue Interpretation dieses neuen Artikels wird sich aber erst in der Ausarbeitung der nächsten Agrarreformen herauskristallisieren.

\subsubsection{Zielkonflikte (Trade-offs)}

Zielkonflikte sind die zentrale Herausforderung, die sich aus einer auf den Begriff Nachhaltigkeit fokussierten Agrarpolitik ergibt. Einerseits ergeben sich Zielkonflikte in Bezug auf die Akteure des landwirtschaftlichen Sektors. Zwischen der wirtschaftlichen Prosperität der Landwirte (möglichst hohe Produzentenrente), preiswerten Nahrungsmitteln (möglichst hohe Konsumentenrente) und tiefen Staatsausgaben für den Steuerzahler ergeben sich grundlegend unterschiedliche Interessen. Die Ausbalancierung dieser Interessen geschieht sowohl über den Markt für Nahrungsmittel als auch über einen politischen Markt (siehe dazu Kapitel 11 zu den agrarpolitischen Prozessen). In Bezug auf die Agrarmärkte sind mit Blick auf die unterschiedlichen Interessen zwei Charakteristiken der Wertschöpfungskette für Nahrungsmittel wichtig. 1) Die Wertschöpfungskette in vielen Ländern weist eine X-Struktur auf (Mérel und Sexton, 2017). Diese Struktur der Akteure führt dazu, dass die Akteure das Machtgleichgewicht entlang der Wertschöpfungskette immer wieder verhandeln. Die Vertreter der Landwirtschaft versuchen dabei auch politisch Einfluss auf die Machtverhältnisse entlang der Wertschöpfungskette zu nehmen (Saitone und Sexton, 2017). 2) Zwar führen langfristige Konsum- und Ernährungstrends zu einer Anpassung der aggregierten Nachfrage nach Lebensmitteln. Die Nachfrage nach Lebensmitteln ist allerdings zumindest kurz- und mittelfristig unelastisch (Dabbert und Braun, 2012). Das bedeutet, dass, auch wenn die Preise für Agrarprodukte sinken, dies nicht zu einer entsprechenden Ausdehnung der Nachfrage führt. Die unelastische Nachfrage kann deshalb zu hohen Preissprüngen führen, die aus einer gesellschaftlichen Perspektive unerwünscht sind. Je nachdem, ob der Preis nach oben oder unten fällt, sind die Landwirte oder Konsumenten stark davon betroffen.

Andererseits ergeben sich Zielkonflikte auch aus der Natur der landwirtschaftlichen Produktion. Landwirtschaftliche Produkte werden grundsätzlich auf natürlichen Böden hergestellt. Auch wenn künstliche Produktionssysteme wie Hors-sol oder Aquakulturen an Bedeutung gewinnen, so wird der Grossteil der Rohprodukte immer noch 
auf landwirtschaftlichen Flächen produziert. Die Bodenabhängigkeit der ursprünglichen landwirtschaftlichen Produktion impliziert, dass automatisch auch ökologische Prozesse von der Landwirtschaft beeinflusst werden (Muller et al., 2017a). Diese Feststellung mutet auf den ersten Blick trivial an. Im Kontext der Agrarpolitik ist dieser Aspekt jedoch von immenser Bedeutung, weil sich in diesem Punkt die Einstellung gegenüber agrarpolitischen Massnahmen herauskristallisiert. Die entscheidende Frage ist, ob ökologische Ziele gemeinsam mit landwirtschaftlichen Produktionszielen erreicht werden sollen (Land Sharing) oder ob es einer Trennung der Zielbereiche bedarf (Land Sparing) (Phalan et al., 2011; Grass et al., 2020). Befürworter einer integralen Zielbetrachtung argumentieren, dass nur eine möglichst ökologische und naturnahe Produktion die Nahrungsmittelproduktion langfristig sichern könne. Vertreter von agrarökologischen Prinzipien des Biolandbaus oder Anhänger einer multifunktionalen Landwirtschaft sehen im Prinzip des «Land Sharing» einen nachhaltigen Ansatz, um auch in Zukunft Lebensmittel zu produzieren. Im Gegensatz dazu argumentieren Vertreter des «Land Sparing»-Ansatzes, dass man intensive Produktionsflächen und solche für die Erreichung ökologischer Ziele räumlich trennen sollte. Dort, wo landwirtschaftliche Flächen knapp sind, wird oft von einem integralen Ansatz ausgegangen. In diesen Systemen entstehen Zielkonflikte zwischen der landwirtschaftlichen Produktion und Umweltzielen wie Erhaltung der Biodiversität, Erosionsschutz, Aufrechterhaltung von Nährstoffkreisläufen oder Schutz vor Verschmutzung (z.B. durch Pflanzenschutzmittel).

Schliesslich kann auch zwischen einer "auf den Markt ausgerichteten Produktion» und dem Ziel einer "sicheren Versorgung" ein gewisser Zielkonflikt bestehen (Möhring et al., 2018). Würde sich die landwirtschaftliche Produktion ausschliesslich auf den Markt ausrichten, würde die Produktion von landwirtschaftlichen Rohstoffen reduziert, die über komparative Kostennachteile verfügen, aber aus einer Perspektive der sicheren Versorgung wichtig sein können. Verschwinden das agronomische Know-how und die dazugehörigen Produktionskapazitäten, kann dies bei einer kurzfristigen Krise zu einem Versorgungsengpass führen. Die Coronapandemie 2020 ist dafür ein gutes Beispiel. Dabei muss aber darauf hingewiesen werden, dass das Konzept einer sicheren Versorgung in wissenschaftlichen und politischen Kreisen umstritten ist. Die grundlegende Frage ist immer, gegen welche Art von Schock oder Krise die Versorgung abgesichert werden soll. Je nach Definition des entsprechenden Szenarios (reduzierter Handel, Wirtschaftskrise, Umweltkatastrophen, Epidemie, Kriege etc.) ändert sich auch der Zielkonflikt zwischen Marktproduktion und Versorgungssicherheit.

Die Agrarpolitik kann echte Zielkonflikte nicht auflösen (siehe dazu auch das Kapitel zur Multifunktionalität der Landwirtschaft). Theoretisch wäre es möglich, dass verbesserte Produktionsverfahren und -technologien die Zielkonflikte mildern. Theore- 
tisch wäre es z.B. sogar denkbar, in einer Hightech-Welt zu leben, in welcher Nahrungsmittel bodenunabhängig produziert würden (Muller et al., 2017a). In naher Zukunft wird sich aber die Agrarpolitik mit den oben beschriebenen Zielkonflikten beschäftigen müssen, indem sie den einzelnen Zielen mehr oder weniger Gewicht zuschreiben.

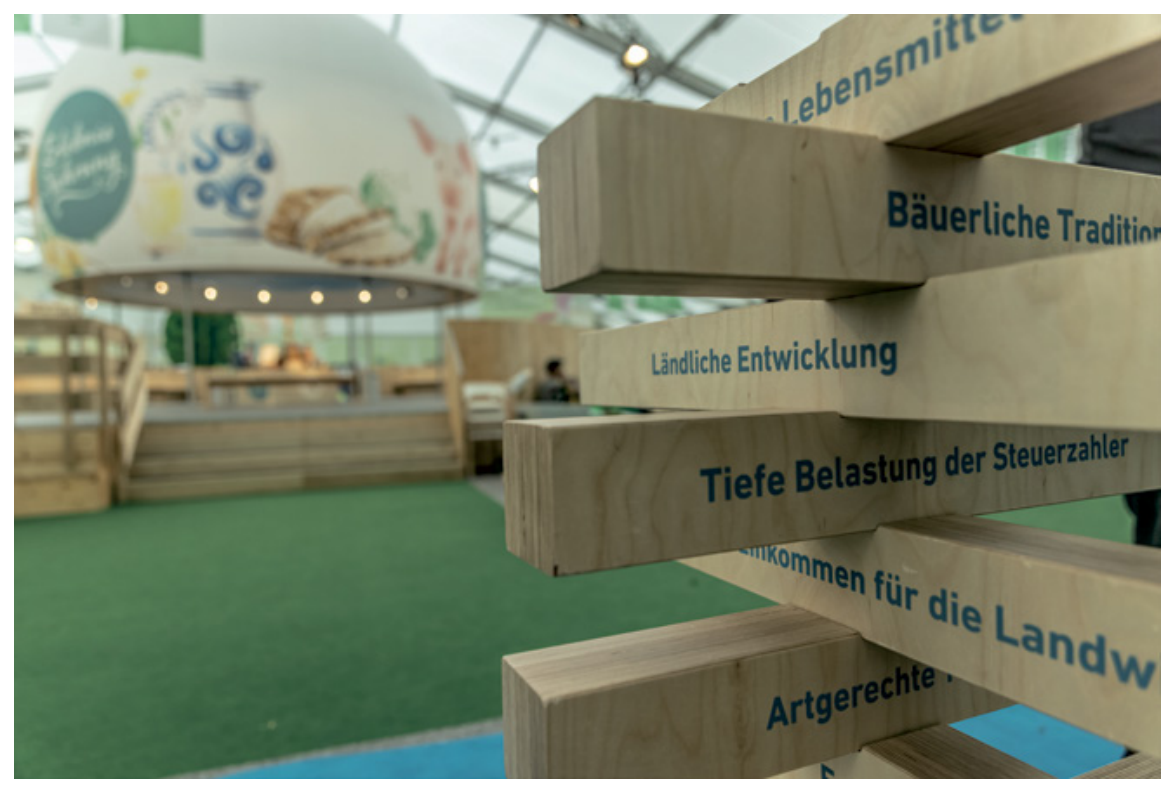

Abbildung 3. Zielkonflikte sind eine zentrale Herausforderung der Agrarpolitik. Die Akteure der Politik müssen agrarpolitische Ziele so gewichten, dass das Landwirtschafts- und Ernährungssystem im Gleichgewicht bleibt. (Bild: Simon Schmid/Smith Art)

In Bezug auf Zielkonflikte und die Wahl von agrarpolitischen Massnahmen wird in diesem Kontext auf die Tinbergen-Regel verwiesen (nach dem Ökonomen Jan Tinbergen). Diese Regel besagt, dass es mindestens so viele agrarpolitische Massnahmen braucht wie Ziele vorhanden sind - aber nicht mehr (Mann und Lanz, 2013). Die Intuition hinter dieser Regel ist einerseits, dass man nicht mit einem Instrument versuchen sollte, mehrere agrarpolitische Ziele zu erreichen. Andererseits impliziert die Regel, dass die Unterstützung eines Zieles mit mehreren Instrumenten gleichzeitig ebenfalls zum Verlust von Ressourcen (z.B. Steuergeldern) führt. Während das erste Prinzip Eingang in die aktuelle Landwirtschaftspolitik der Schweiz gefunden hat (im Rahmen der Weiterentwicklung des Direktzahlungssystems, WDZ), ist die Frage nach der Anzahl Instrumente pro Ziel eher umstritten. Grund dafür ist wiederum die Koppelproduktion von verschiedenen Leistungen in der Landwirtschaft (siehe dazu das Kapitel zur Jointness). Konkret stellt sich beispielsweise die Frage, ob es in der 
Schweiz eine Direktzahlung für den Biolandbau braucht, wo doch Biodiversität, Landschaftspflege, graslandbasierte Milch- und Fleischproduktion - also konstituierende Elemente des Biolandbaus - bereits abgegolten werden (Schader et al., 2014).

\subsection{Grundlagen einer Agrarmarktordnung}

Grundsätzlich ist die Agrarpolitik ein staatlicher Eingriff in die Agrarmärkte (siehe auch Kapitel 2). Sie regelt dadurch die Rahmenbedingungen für die landwirtschaftliche Produktion und den dazugehörigen Märkten. Das Zusammenspiel aus Märkten und politischen Eingriffen kann als Agrarmarktordnung verstanden werden. Mit einem ordnungspolitischen Blick, d.h. der Perspektive, dass die Wirtschaft in erster Linie nach den Prinzipien von Markt und Wettbewerb zu organisieren seien, braucht es eine Begründung für staatliche Eingriffe. Um die Agrarpolitik konzeptionell zu verstehen, ist es wichtig, die folgenden Begriffe Marktversagen, Politikversagen und Marktmacht zu definieren.

\subsubsection{Marktversagen}

In einer rationalen Agrarpolitik, die sich auf eine marktwirtschaftliche Ordnung beruft, ist ein Marktversagen die Bedingung, um staatliche Eingriffe rechtfertigen zu können (Perman et al., 2003). Im Umkehrschluss bedeutet dies, dass Politikmassnahmen dann sinnvoll sind, wenn eine Koordination über den Markt nicht zu einer optimalen Allokation der knappen Ressourcen führt (optimal im Sinne der Wohlfahrtsökonomie; Paretoeffizienz). Die Gründe für eine nicht wohlfahrtsmaximierende Allokation der Ressourcen können vielfältig sein (Perman et al., 2003). Die häufigsten Ursachen sind das Vorhandensein von externen Effekten und öffentlichen Gütern (siehe nächstes Unterkapitel), unvollständiger Wettbewerb (z.B. Monopol oder Kartelle), Unsicherheit und Nichtumkehrbarkeit im Falle von Anpassungsmängeln oder Strukturkrisen, fehlende oder ungenügende Eigentumsrechte (Allmendgüter) oder Informationsasymmetrien wie Adverse Selection oder Moral Hazard. Marktversagen ist aber lediglich ein notwendige, nicht jedoch eine hinreichende Bedingung, um Eingriffe des Staates zu rechtfertigen. Aus einer wohlfahrtsökonomischen Sicht müssen staatliche Eingriffe auch zu einer Verbesserung der Ressourcenallokation führen. Wichtig ist hier anzuführen, dass das Prinzip der Paretoeffizienz unterschiedliche Verteilungswirkungen haben kann. Das bedeutet, dass ein wohlfahrtsökonomisches Optimum noch nichts über die Gewinner und Verlierer aussagt (Stiglitz, 2007). Es bedeutet aber, dass jemand bessergestellt werden könnte, ohne dass ein anderer Akteur schlechter dastehen würde. Gemäss der ökonomischen Theorie sollte ein Ausgleich zwischen sozialen Akteuren über Steuern und Transfers erfolgen und nicht über die Allokation von Boden, Arbeit oder Kapital (Samuelson, 1954). Die Vorstellung, dass ein solcher Transfer in jedem Fall machbar und auch gerecht ist, bleibt aber auch innerhalb der Ökonomie umstritten (Sen, 2018). 


\subsubsection{Politikversagen}

Trotzdem ist die Definition des Marktversagens wichtig, weil durch die hinreichende Bedingung - eine Paretoverbesserung - auch das Konzept des Politikversagens definiert werden kann (Perman et al., 2003). Das bedeutet, dass staatliche Eingriffe auch zu Fehlanreizen führen können und damit eine optimale Allokation von Ressourcen beeinträchtigen (auch hier: immer im Sinne der Wohlfahrtsökonomie). Die Gründe für Politik- oder Staatsversagen können analog zum Marktversagen vielfältig sein. Klassische Ursachen sind die Natur des Politiksystems (z.B. Diktatur), Einfluss von Lobbying oder der Bürokratie (siehe Kapitel 9), Principle-Agent-Problematik (d.h., der Bauer hat einen Wissensvorsprung gegenüber dem regulierenden Staat und kann sich dieses Wissen zu eigen machen) oder undifferenzierte Politikmassnahmen (One Size Fits All), die dazu führen können, dass für Leistungen staatliche Gelder eingesetzt werden, die auch ohne diese erbracht würden (Mitnahmeeffekte). Schliesslich können Komplexität oder Zielkonflikte dazu führen, dass gar keine optimale Regulierung möglich ist. In diesem Fall müssen die Gewinne und Verluste gegeneinander abgewogen und Massnahmen umgesetzt werden, die zu einer Minimierung der Verluste führt (Second-Best Theory) (Baumol und Oates, 1988). Es existiert jedoch keine einheitliche Definition für staatliches Versagen, weil die Art des Staatsversagens wesentlich von der Natur des Marktversagens beeinflusst wird.

\subsubsection{Marktmacht}

Eine dritte wichtige Definition für das Verständnis von Agrarmarktordnungen ist der Begriff der Marktmacht. Aus einer theoretischen Sicht existieren in den unterschiedlichen Wirtschaftssektoren unterschiedliche Marktstrukturen und Marktformen. Die spezifische Struktur der Wertschöpfungskette im Ernährungssektor und die damit verbundene Marktmacht sind zentrale Argumente im Kontext der Agrarpolitik. Der Nahrungsmittelsektor wird oft durch eine sogenannte X-Struktur charakterisiert (Mérel und Sexton, 2017; Saitone und Sexton, 2017). Das bedeutet, dass viele Produzenten an wenige Verarbeiter und Händler liefern, die wiederum einzelne Detailhändler beliefern. Letztere wiederum verkaufen ihre Produkte an viele Konsumenten. In der Schweiz ist der Sektor zusätzlich durch eine relativ hohe Integration der Wertschöpfungskette geprägt (Bokusheva et al., 2019; Gentile et al., 2019; Mann et al., 2019). Viele wichtige Verarbeiter wie beispielsweise Bell und Micarna in der Fleischverarbeitung, die Swissmill, die Coop Bäckerei und Jowa in der Getreideverarbeitung oder die Elsa in der Milchverarbeitung sind Unternehmen der Coop bzw. der Migros, den beiden grössten Detailhändlern in der Schweiz. Schätzungen zufolge decken Coop und Migros mehr als $70 \%$ des Marktes für Nahrungsmittel ab. Auch die Fenaco ist ein marktmächtiger Akteur, der einerseits viele Produktionsmittel zur Verfügung stellt (z.B. UFA Samen, Landor, Melior). Andererseits ist die Fenaco ein bedeutender Händler und Abnehmer von landwirtschaftlichen Produkten (z.B. Anicom, Ernst Sutter AG, Fenaco Landesprodukte). 
Theoretisch lassen sich Märkte in unterschiedliche Marktformen unterscheiden (Varian, 2016). In einem Monopol beispielsweise stehen viele Nachfrager einem grossen Anbieter gegenüber. Dieser kann auf dem Markt Mengen und Preise festlegen, was dazu führt, dass jeweils eine kleinere Menge zu höheren Preisen abgesetzt wird (Monopolrente). Umgekehrt wird in einer Situation mit vielen kleinen Anbietern und einem grossen Abnehmer von einem Monopson gesprochen. In einem Oligopol stehen mindestens zwei oder wenige Anbieter vielen Nachfragern gegenüber. In der Schweiz mit den grossen Akteuren Coop und Migros sowie den kleineren Aldi und Lidl kann man beispielsweise von einem Teiloligopol sprechen. Ein Markt mit wenigen Abnehmern und vielen Anbietern wird als Oligopson bezeichnet. Welche Auswirkung ein Oligopol bzw. ein Oligopson auf Mengen und Preisbildung hat, hängt von den strategischen Zielen der Marktteilnehmer ab, die sich beispielsweise für eine Preisoder Mengenführerschaft entscheiden. Theoretisch ist zu erwarten, dass a) die Konsumentenpreise tiefer und die Produzentenpreise höher sind als unter Bedingungen im Monopol/Monopson, aber b) die Konsumentenpreise höher und die Produzentenpreise für die Landwirtschaft tiefer sind als bei einem vollkommenen Markt, in welchem viele Nachfrager vielen Anbietern gegenüberstehen.

Es ist wichtig zu betonen, dass die Marktmacht innerhalb der Wertschöpfungskette für Nahrungsmittel auch eine Folge des Grenzschutzes ist, der den Markteintritt für ausländische Unternehmen erschwert und damit die Marktstruktur zementiert. In der Schweiz wird zudem über das Kartellrecht sichergestellt, dass die Akteure entlang der Wertschöpfungskette keine zu grosse Marktmacht erhalten. Ausserdem sollen Branchen- und Produzentenorganisationen dafür sorgen, dass die Marktmacht der Abnehmer nicht allzu stark ausgenutzt werden kann. Es ist auch darauf hinzuweisen, dass viele Akteure innerhalb der Wertschöpfungskette, wie beispielsweise die Fenaco, genossenschaftlich organisiert sind und die Produzentinnen und Produzenten darin eine wichtige Rolle spielen.

\subsection{Multifunktionalität}

\subsubsection{Multifunktionalität der Landwirtschaft}

Im europäischen Kontext manifestiert sich der Begriff der Multifunktionalität als Schlüsselbegriff für die Rechtfertigung der Stützung des Landwirtschaftssektors (Huber, 2010). Im Kern besagt Multifunktionalität, dass die Landwirtschaft nicht nur Nahrungsmittel produziert, sondern gleichzeitig zusätzliche Funktionen erbringt, welche von der Gesellschaft nachgefragt werden (siehe Box 2).

Der Begriff Multifunktionalität wurde im politischen Diskurs im Laufe der UruguayRunde populär (OECD, 2001). Die G-10-Länder argumentierten, dass neben der Produktion von agrarischen Rohstoffen die Landwirtschaft noch weitere Leistungen erbringt, die den Charakter von Externalitäten oder öffentlichen Gütern aufwiesen. 


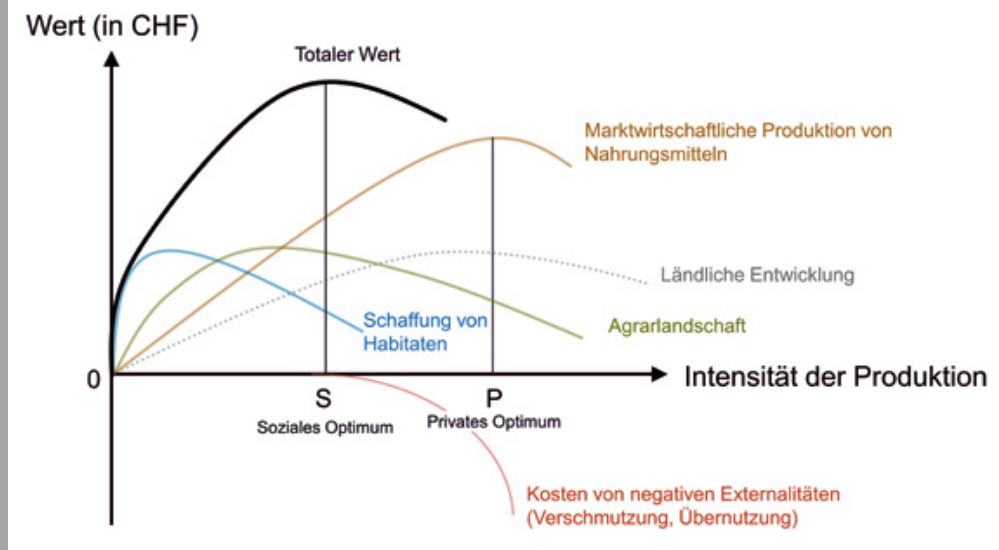

Quelle: in Anlehnung an Harvey 2003

Stünde für die Landwirtschaft nur die marktwirtschaftliche Produktion von Nahrungsmitteln im Zentrum, dann wäre es aus einer ökonomischen Perspektive effizient, im privaten Optimum zu produzieren (d.h. im Punkt P). Das bedeutet, dass, solange der Einsatz von zusätzlichen Inputfaktoren den Wert der Nahrungsmittelproduktion steigert, so lange wird die landwirtschaftliche Produktion auch intensiviert. Diese Zunahme der Intensität kann aber zu einer Übernutzung führen und negative Externalitäten verursachen (rote Kurve). Gleichzeitig entstehen durch die landwirtschaftliche Nutzung aber auch von der Gesellschaft erwünschte Leistungen wie beispielsweise die Schaffung von Habitaten für die Erhaltung der Biodiversität (blaue Kurve) oder die Pflege der Kulturlandschaft (grüne Kurve). Diese zusätzlichen Leistungen sind normalerweise höher, wenn die Intensität der Nutzung tiefer ist. Wiederum andere Leistungen sind stärker mit der landwirtschaftlichen Produktion verbunden wie beispielsweise der Beitrag zur ländlichen Entwicklung (graue Kurve). Betrachtet man nun alle die Leistungen der Landwirtschaft zusammen, dann ergibt sich ein totaler Wert, dessen Optimum sich in diesem Fall links vom privaten Optimum befindet (S). Wo genau dieses soziale Optimum zu liegen kommt, hängt von der Art der Produktion und der Nachfrage der Gesellschaft ab (d.h. wie viel Wert sie den einzelnen Funktionen beimisst). Diese ganzheitliche Betrachtung der Landwirtschaft basierend auf den unterschiedlichen Funktionen wird als Multifunktionalität bezeichnet. 
Um diese zusätzlichen Leistungen aufrechtzuerhalten, sei es legitim, die Handelsfreiheit einzuschränken (Abler, 2004; Van Huylenbroeck et al., 2007).

In der Schweizer Agrarpolitik beschreibt der Artikel 104 der Bundesverfassung, was unter dem Begriff Multifunktionalität verstanden werden soll (Flury und Huber, 2007). In der Wissenschaft wird der Begriff erst seit den 1990er-Jahren verwendet, obwohl die Prinzipien der Multifunktionalität schon sehr viel älter sind. Die gleichzeitige Entwicklung des Begriffs in Politik und Wissenschaft verursachte Unschärfe in dessen Definition und Nutzung (Hediger und Knickel, 2009). In der WTO beispielsweise wird der Begriff der Multifunktionalität nicht direkt verwendet, sondern mit «Non-Trade Concerns» umschrieben. Die FAO verwendet den Begriff der Multifunktionalität ebenfalls nicht und nutzt «Roles of Agriculture», um zu beschreiben, dass die Landwirtschaft nicht nur Rohstoffe produziert, sondern weitere ökologische und soziale Leistungen erbringt.

Um eine Agrarpolitik, die sich auf den Grundsatz der Multifunktionalität beruft, aus einer rationalen Perspektive beurteilen zu können, bietet sich die Definition der OECD an, welche die agrarökonomische Umschreibung «Koppelproduktion und Marktversagen» (Jointness and Market Failure) als Definition für Multifunktionalität verwendet (OECD, 2001).

\subsubsection{Marktversagen im Kontext des Agrarsektors}

Damit sich eine politische Intervention in die Landwirtschaft unter dem Begriff Multifunktionalität rechtfertigen lässt, ist es zwingend, dass ein Marktversagen vorliegt. Im Kontext der Landwirtschaft sind dabei in erster Linie drei Aspekte gemeint.

1. Externalitäten mit dem Charakter von öffentlichen Gütern. Externalitäten sind Auswirkungen ökonomischer Entscheidungen auf unbeteiligte Dritte, ohne dass eine Kompensation, z.B. durch den Marktpreis, stattfindet. Man spricht in diesem Zusammenhang auch von nicht marktfähigen oder gemeinwirtschaftlichen Leistungen. Es gibt sowohl positive, z.B. die Pflege einer kleinstrukturierten Landschaft, als auch negative Externalitäten, z.B. die Einbringung von Pflanzenschutzmittel in die Umwelt (Power, 2010). Die Landwirtschaft würde ohne staatliche Steuerung weniger positive Externalitäten anbieten, als es dem wohlfahrtstheoretischen Optimum entspricht. Umgekehrt würden, ohne staatliche Regulierung, mehr negative Externalitäten in Boden, Wasser und Luft eingebracht, als von der Gesellschaft gewünscht. Für eine wohlfahrtstheoretisch optimale Allokation der Produktionsfaktoren wäre es daher notwendig, Externalitäten in den Wirtschaftskreislauf und damit die Produktionsentscheide der Landwirte zu internalisieren (Hodge, 2002; Harvey, 2003). Theoretisch wäre dies auch ohne staatliche Intervention möglich. Das sogenannte CoaseTheorem (benannt nach dem Ökonomen Ronald Coase) besagt, dass eine Festlegung von Eigentumsrechten und sich daraus ergebende Verhandlungen zwischen dem Ver- 
ursacher und dem Betroffenen von Externalitäten zu einer optimalen Allokation führen kann (Hendrikse, 2003; Perman et al., 2003), allerdings nur dann, wenn sämtliche Informationen und keine Transaktionskosten vorliegen (Sterner et al., 2019).

Im Gegensatz zur industriellen Produktion basiert die landwirtschaftliche Produktion unmittelbar auf Agrarökosystemen und ist damit abhängig von natürlichen Prozessen wie Bodenbildung, dem Nährstoffkreislauf oder dem Klima. In Bezug auf die Landwirtschaft haben die entsprechenden Externalitäten deshalb oft auch den Charakter von öffentlichen Gütern. Öffentliche Güter sind durch zwei Eigenschaften charakterisiert (Varian, 2016): 1) Nicht-Ausschliessbarkeit, d.h., es kann niemand vom Konsum des Gutes ausgeschlossen werden. 2) Nicht-Rivalität im Konsum, d.h., das Gut wird beim Konsum nicht verbraucht, sodass viele Menschen es gleichzeitig nutzen können. Klassische Beispiele für Externalitäten mit dem Charakter von öffentlichen Gütern in der Landwirtschaft sind a) Erhaltung der Biodiversität, Landschaftspflege oder die Erhöhung des Tierwohls (positive Externalitäten) und b) der Eintrag von Stickstoff und Pflanzenschutzmittel in die Umwelt oder Erosion (negative Externalitäten). In der Agrarpolitik wird oft auch die Verhinderung von negativen Externalitäten durch spezifische Produktionsmassnahmen oder -technologien (z.B. eine Verminderung der Stickstoffauswaschung durch den Einsatz eines Schleppschlauchsystems) als positive Externalität dargestellt. Die Interpretation hängt dabei von der Definition der Eigentumsrechte ab. Haben die Landwirte ein Recht auf Verschmutzung ihres privaten Eigentums oder hat die Gesellschaft per se Anrecht auf sauberes Trinkwasser? In welchem Ausmass somit eine Externalität als positiv oder negativ bewertet wird, hängt in der Agrarpolitik oft davon ab, wie man diese Eigentumsrechte definiert (Bromley, 2000; Dasgupta, 2021).

2. Gesellschaftlich erwünschte Bereitstellung von privaten Gütern. Neben den klassischen öffentlichen Gütern können zusätzliche Leistungen der Landwirtschaft auch den Charakter von sogenannten meritorischen Gütern haben (Mann, 2006b). Dabei handelt es sich um private Güter, oder zumindest nicht um rein öffentliche Güter, die ohne staatlichen Eingriff, aber aus gesellschaftlicher Sicht in zu geringem Ausmass oder nicht für alle sozialen Schichten angeboten würden. Klassische Beispiele sind die Versorgungssicherheit und die dezentrale Besiedlung. Diese beiden Leistungen sind per Definition keine öffentlichen Güter. Man könnte die Entscheidung, inwiefern er oder sie sich für Zeiten mit gestörter Versorgung vorbereiten soll, jedem einzelnen Bürger überlassen. Auch die dezentrale Besiedlung erfüllt das Kriterium der NichtAusschliessbarkeit nicht. Es wäre sehr wohl möglich, einzelne Regionen aufzugeben und Rückzugsräume für die Wildnis zu schaffen. Beide Leistungen sind aber von der Gesellschaft erwünscht, weil sie sozialen Ausgleich und Zusammenhalt schaffen und damit ein konstituierendes Element der Gesellschaft bzw. der Nation sein können. In anderen Ländern, wie beispielsweise den USA oder Australien, spielen meritorische Güter bzw. die Multifunktionalität per se keine Rolle in der Agrarpolitik (siehe dazu 
auch Kapitel 6). Diese Länder wehren sich auch gegen den Begriff der Multifunktionalität und bevorzugen ein "Land Sparing» für die Aufrechterhaltung von ökologischen Funktionen oder Leistungen (Anderson, 2000).

3. Landwirtschaft und Produktion im ländlichen Raum. Ein dritter wichtiger Bereich des Marktversagens, der dem Begriff Multifunktionalität innewohnt, ist die soziale Dimension einer bäuerlichen Landwirtschaft (Janker und Mann, 2020). Aus Sicht der "Multifunktionalisten» ist eine nachhaltige Landwirtschaft eng an den Familienbetrieb geknüpft. Die Landwirtschaft wird als mehr verstanden als nur ein reiner Wirtschaftszweig. Traditionelle Wirtschaftsweisen und familiäre Produktionsstrukturen auch in ländlichen und Bergregionen - sollen einen wichtigen Beitrag leisten zur dezentralen Besiedlung und für multifunktionale Landschaften (Collier, 2011); dies als Kontrast zu einer stärker an die Prinzipien der Industrie (Economies of Scale, Effizienz, Handel) ausgerichteten Landwirtschaft wie in den USA oder Australien. Die Zerstörung von lokalen und integrierten Produktionsstrukturen in ländlichen Regionen wird aus dieser Sicht als Marktversagen interpretiert, das einen staatlichen Eingriff rechtfertigt.

\subsubsection{Jointness (Koppelproduktion) von marktfähigen und nicht marktfähigen Gütern}

Neben einer Form von Marktversagen ist die zweite Voraussetzung für ein staatliches Eingreifen unter dem Begriff der Multifunktionalität, dass die Erbringung der nicht marktfähigen Leistungen an die landwirtschaftliche Produktion geknüpft ist (Flury und Huber, 2007). Man spricht in diesem Zusammenhang von Koppelproduktion (Jointness). Die Koppelproduktion ist ein Bestandteil vieler Wirtschaftsbereiche und ist nicht ein spezifisches Charakteristikum der Landwirtschaft. Oft handelt es sich dabei aber um die Kopplung von privaten Gütern. In der Landwirtschaft gehört dazu z.B. die Produktion von Wolle und Fleisch beim Schaf oder die Produktion von Milch und Fleisch in Milchbetrieben. Der zentrale Aspekt für die Multifunktionalität ist nun, dass viele Koppelprodukte der Landwirtschaft nicht marktfähig sind. Diese Koppelproduktion von marktfähigen und nicht marktfähigen Gütern in der Landwirtschaft basiert auf drei unterschiedliche Ursachen (Abler, 2004; Huber und Lehmann, 2010):

1. Technische Interdependenzen. Die Produktion von Nahrungsmitteln ist «produktionstechnisch» mit der Bereitstellung von externen Effekten verbunden. Beispiel: Mit der Milchproduktion sind $\mathrm{CH}_{4}$-Emissionen verbunden. Mit dem Anbau von Leguminosen wird Stickstoff fixiert.

2. Nicht zuweisbare variable Inputs. Ein variabler Input aus der landwirtschaftlichen Produktion (z.B. Boden) ist mit einer Externalität oder einem öffentlichen Gut verbunden. Beispiel: Die Nutzung von Flächen im Berggebiet führt automatisch zur Kulturlandschaft.

3. Nicht zuweisbare fixe Faktoren. Die Erbringung von öffentlichen Gütern durch die Landwirtschaft wird durch die fixen Faktoren auf Betriebsebene 
restringiert. Beispiel: Die Bereitstellung von Biodiversitätsförderflächen (BFF) wird durch die Futterknappheit im Betrieb mitbestimmt.

Die beiden letzteren Ursachen können unter dem Terminus «Ökonomische Interdependenz» zusammengefasst werden (Abler, 2004). Je nachdem, ob die Koppelproduktion auf eine technische oder eine ökonomische Interdependenz zurückzuführen ist, eignen sich andere Politikinstrumente zur Unterstützung einer multifunktionalen Landwirtschaft (siehe dazu das nächste Kapitel). Die technischen und ökonomischen Interdependenzen in bäuerlichen Familienbetrieben, wie sie in Mitteleuropa vorherrschen, resultieren aber in äusserst komplexen Beziehungen zwischen der Produktion von marktfähigen (z.B. Nahrungsmittel) und nicht marktfähigen Gütern (z.B. Landschaft), welche eine sorgfältige Trennung der Ursachen erschweren. Mit anderen Worten, theoretisch können die Ursachen der Jointness festgelegt werden. Die realen Produktionssysteme sind aber zu divers, um in jedem Fall eindeutige Zusammenhänge zu etablieren (Huber et al., 2017a).

Darüber hinaus ist es relativ einfach, nicht marktfähige Leistungen von der landwirtschaftlichen Produktion zu trennen. Biodiversität oder Arbeit im ländlichen Raum könnten theoretisch ja auch von anderen, nicht landwirtschaftlichen Akteuren erbracht werden, z.B. von einem Betreiber eines Golfplatzes oder von staatlichen Landschaftspflegern. Die Frage, ob die Multifunktionalität der Landwirtschaft als Rechtfertigung für deren Stützung genutzt werden kann, ist daher nicht nur eine konzeptionelle, sondern auch eine empirische Frage (Van Huylenbroeck et al., 2007). Für deren Beantwortung wird Bezug auf ein anderes ökonomisches Konstrukt genommen: Economies of Scope. Die Economies of Scope beschreiben den Umstand, dass die gleichzeitige Produktion von zwei Gütern kostengünstiger ist, als wenn die beiden Güter unabhängig voneinander bereitgestellt werden (Huber und Lehmann, 2010). Erst der Umstand, dass die Jointness zu Economies of Scope und damit tieferen Kosten für die Bereitstellung von nicht marktfähigen Leistungen führt, rechtfertigt aus einer rationalen Perspektive die Unterstützung der Landwirtschaft durch die Politik. Wenn die Erbringung der nicht marktfähigen Leistungen wie Biodiversität oder Landschaftspflege kostengünstiger von anderen Akteuren erbracht werden könnten, dann müssten auch diese nicht landwirtschaftlichen Akteure als mögliche Leistungserbringer berücksichtigt werden, z.B. indem die Leistungen ausgeschrieben würden (OECD, 2003).

Der Begriff der Koppelproduktion/Jointness scheint im Kontext der Schweizer Landwirtschaft auf den ersten Blick trivial. Sie hat aber aus einer konzeptionellen Perspektive eine elementare Bedeutung für die Rechtfertigung der landwirtschaftlichen Stützung auf der Basis des Begriffs Multifunktionalität: Wenn die nicht marktfähigen Leistungen wie Biodiversität oder Landschaftspflege auch von nicht landwirtschaftlichen Akteuren erbracht werden können, dann erschliesst sich nicht, weshalb ausgerechnet nur die Landwirtschaft unterstützt werden sollte. Über den Begriff der Economies of Scope/Verbundeffekte wird die Koppelproduktion operationalisiert. Sie sind der 
Kern der Unterstützung der landwirtschaftlichen Bereitstellung von öffentlichen Gütern und damit der Multifunktionalität in der Schweizer Agrarpolitik.

\subsubsection{Entkopplung (Decoupling)}

Ein weiterer kritischer Begriff aus dem Kontext der rationalen Agrarpolitik ist das Decoupling (Entkopplung). Im Kern besagt das Decoupling, dass eine agrarpolitische Massnahme oder ein Set von Massnahmen keinen oder einen minimalen Effekt auf die Produktion und den Handel von Agrarprodukten hat (OECD, 2006; Chambers und Voica, 2017).

Die Erreichung der agrarpolitischen Ziele durch die Agrarpolitik fokussierte bis zur Uruguay-Runde auf die Beeinflussung der Märkte und die Steuerung der Produktpreise für die Landwirte (z.B. Zölle, Abnahmegarantien, Exportsubventionen). Mit dem Agreement on Agriculture musste die Stützung Green Box tauglich umgebaut werden. Die grundlegende Idee dahinter war, die Ziele zur Unterstützung der Einkommen von den preispolitischen Massnahmen zu trennen und so die Unterstützung der Landwirtschaft möglichst markt- und produktionsneutral zu gestalten. In der agrarökonomischen Forschung wurde die Trennung der Preis- von der Einkommenspolitik (= Decoupling) schon in den 1970er-Jahren propagiert. In der Schweiz wurde diese Trennung theoretisch mit der Annahme des neuen Artikels 104 in der BV (1996) und der Einführung von Direktzahlungen umgesetzt. In der EU wurden Direktzahlungen mit der Fischler-Reform im Jahr 2000 eingeführt und bis 2006 in den Ländern unterschiedlich umgesetzt (Kazukauskas et al., 2013; Erjavec und Lovec, 2017).

Die theoretische Grundlage des Decouplings beinhaltet, dass die Stützung der Landwirtschaft durch die Beeinflussung der Preise zu wohlfahrtstheoretischen «Dead weight losses» führt. In der ökonomischen Theorie wird die Wirkung einer Politikmassnahme mithilfe des Rentenansatzes beurteilt (Alston und James, 2002). Die Box 3 zeigt, was mit dem Rentenansatz gemeint ist. In einem vollkommenen Markt bildet sich der Preis für Agrargüter dort, wo sich Angebot (die horizontal aggregierte Grenzkostenkurve der Landwirte) und Nachfrage (die horizontale Aggregation der individuellen Zahlungsbereitschaft der Konsumenten) schneiden. Achtung: Im Gegensatz zu einem privaten Gut werden bei einem öffentlichen Gut die individuellen Zahlungsbereitschafen vertikal aggregiert (siehe Kapitel 11). Zwischen dem Marktpreis im Gleichgewicht und der Zahlungsbereitschaft der Konsumenten ergibt sich die sogenannte Konsumentenrente. Sie ist die Summe der Zahlungsbereitschaften derjenigen Konsumenten, die auch bereit gewesen wären, einen höheren Produzentenpreis zu bezahlen. Umgekehrt fasst die Produzentenrente denjenigen Gewinn der Landwirte zusammen, der zwischen der Angebotskurve und dem Marktpreis liegt, d.h. derjenigen Landwirte, die auch zu tieferen Preisen kostendeckend produzieren können. Politikmassnahmen, die den Marktpreis zugunsten der Produzentenrente erhöhen, führen im Markt zu einer Fehlallokation der Inputfaktoren und zu Wohlfahrtsverlusten. 
Einführung in die Schweizer Agrarpolitik

Box 3 Die Konsumenten- und Produzentenrente

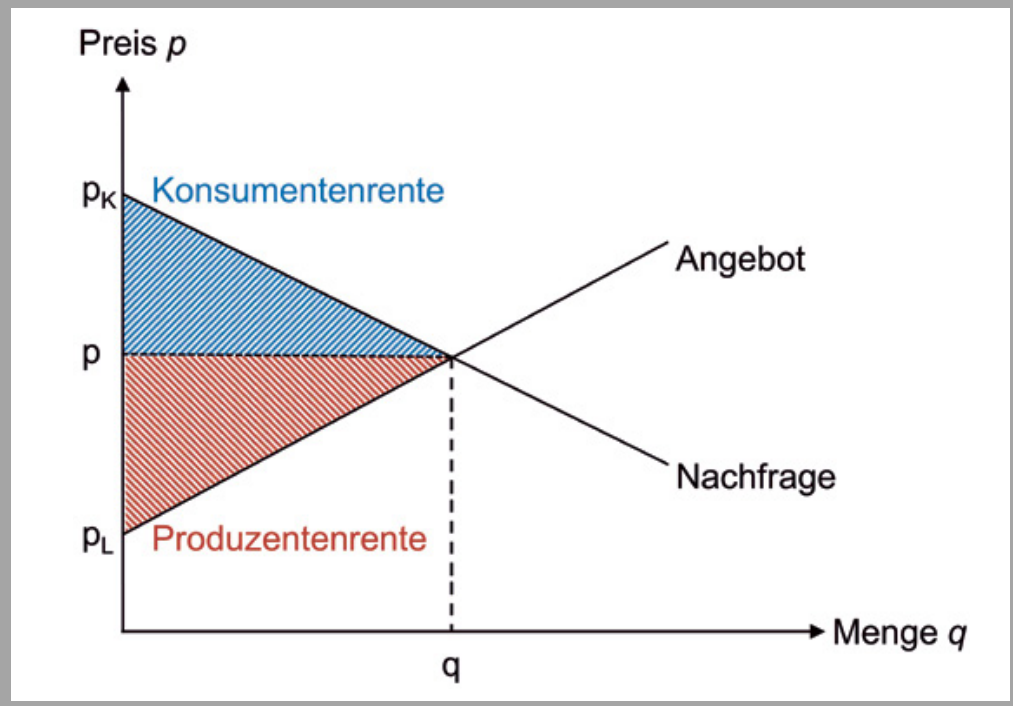

Im Marktgleichgewicht schneiden sich die Angebots- und Nachfragefunktion und die Menge q wird zum Preis p verkauft. Die Nachfrage entsteht aus der Aggregation der individuellen Zahlungsbereitschaften der Konsumenten. Die Zahlungsbereitschaft der Konsumenten leitet sich aus der individuellen Nutzenmaximierung bei gegebenem Budget ab. Je höher der Preis, desto geringer die nachgefragte Menge.

Umgekehrt besteht die Angebotsfunktion aus der Aggregation der Produktion der Landwirte. Diese leitet sich aus den Grenzkosten der Produzenten ab. Je höher der Preis, desto grösser die hergestellte Menge. Der Preis $p_{k}$ stellt die maximale Zahlungsbereitschaft der Konsumenten dar. Im Marktgleichgewicht entsteht eine Differenz zwischen der maximalen Zahlungsbereitschaft der Konsumenten und dem Gleichgewichtspreis. Diese Differenz wird als Konsumentenrente bezeichnet. Es ist der Nutzengewinn für die Konsumenten, die eigentlich eine höhere Zahlungsbereitschaft hätten. Umgekehrt wird die Differenz zwischen Angebotsfunktion und dem Marktpreis als Produzentenrente bezeichnet. Die Produzenten erzielen eine Rente, weil der Preis höher ist als die Grenzkosten der Produktion. Die Beurteilung der Wohlfahrt, ein Mass für den gesamten Nutzen, erfolgt über die Addition dieser beiden Renten. 
Die Logik des Decoupling ist, dass man die wohlfahrtsökonomischen Verluste zu vermeiden versucht, indem man die Marktkräfte über Angebot und Nachfrage den Preis bestimmen lässt und die ökonomischen Verluste der Produzenten über direkte Transfers aus diesen Wohlfahrtsgewinnen kompensiert (Chambers, 2002; Matthews, 2013). Diese Kompensation findet meist über Direktzahlungen an die Landwirte statt, welche aus Steuereinnahmen finanziert sind. Mit anderen Worten verschiebt das Decoupling den Aufwand für die Stützung der Landwirtschaft von den Konsumenten zu den Steuerzahlern. Der Gewinn resultiert aus den vermiedenen Wohlfahrtsverlusten.

Die Verbindung der Direktzahlungen mit der Multifunktionalität schafft die Rechtfertigung für die Belastung der Steuerzahler. Theoretisch hat das Decoupling also grosse Vorteile. Die Umsetzung schafft aber auch praktische Schwierigkeiten:

1) Die Abgeltung der Landwirte für die Erbringung von öffentlichen Leistungen reicht unter Umständen nicht aus, um den Verlust der Produzentenrente am Markt zu kompensieren. In der agrarpolitischen Realität wurden die Direktzahlungen jeweils so ausgestaltet, dass sie Verluste auf den Märkten möglichst kompensierten - unabhängig vom Wert oder Ausmass der öffentlichen Güter. Da der Wert öffentlicher Leistungen nur schwer zu bestimmen ist (siehe dazu Bewertungsfragen aus der Ressourcenökonomie z.B. [Perman et al., 2003]), kann dies als agrarpolitischer Pragmatismus betrachtet werden. Es schafft aber einen argumentativen Keil zwischen den konzeptionellen Grundlagen der Multifunktionalität und der Implementierung des Decouplings. Dieser Keil entspricht den Mitnahmeeffekten, die durch die Stützung der Landwirtschaft entstehen. Von Mitnahmeeffekten spricht man, wenn die Landwirte finanziell entschädigt werden für etwas, das sie auch ohne Stützung tun würden. Mitnahmeeffekte reduzieren die Effizienz einer Politikmassnahme erheblich und können im Extremfall dem Politikversagen zugeordnet werden, weil sich die Ressourcenallokation verschlechtert. Die Frage, wie hoch die Abgeltung der Landwirte für die Erbringung öffentlicher Leistungen sein soll, wurde und wird ständig neu evaluiert und politisch verhandelt. Dabei spielen aber nicht nur Effizienz-, sondern auch Effektivitätsüberlegungen und Verteilungseffekte eine zentrale Rolle (Mann, 2006a).

2) Direkte Einkommenstransfers wirken sich auf den Agrarstrukturwandel aus. Es ist zu erwarten, dass Direktzahlungen dazu führen, dass weniger Betriebe aus der landwirtschaftlichen Produktion aussteigen und mehr Betriebe übernommen werden als unter reinen Marktbedingungen. Ist der Transfer an Flächen gebunden, wie im Schweizer Direktzahlungssystem, reduzieren sich auch die Aktivitäten auf dem landwirtschaftlichen Bodenmarkt. Weil die Direktzahlungen zu höheren Bodenrenten und damit höheren Pacht- und Kaufpreisen führen, wird die Strukturentwicklung hin zu grösseren Betrieben gehemmt. Ausserdem können höhere Pachtpreise den Eigentümern der Flächen zugutekommen und weniger den eigentlichen Bewirtschaftern. In der Schweiz ist rund die Hälfte der landwirtschaftlichen Nutzflächen gepachtet. 
Der Boden- und Pachtlandmarkt ist allerdings stark reguliert. Empirisch hat sich gezeigt, dass die Direktzahlungen nur einen kleinen Einfluss auf höhere Pacht- und Bodenpreise haben (Graubner, 2017).

3) Aus einer konzeptionellen Perspektive sollte das Decoupling zu einer produktionsunabhängigen Stützung führen. In der Praxis kann der Einkommenstransfer aber zu sogenannten Versicherungseffekten (der Betrieb produziert mehr, weil das Marktrisiko durch die Transfers abgefedert wird), Vermögenseffekten (der Betrieb produziert mehr, weil er mehr Kapital zur Verfügung hat) und Erwartungseffekten (Betriebe passen ihre Produktion laufend an, um beispielsweise die Referenzzahlung zu erhöhen) führen (Moro und Sckokai, 2013; Chambers und Voica, 2017).

Trotz dieser Schwierigkeiten in der praktischen Umsetzung des Decouplings ist dieses Konzept das Kernstück der Verbindung zwischen der Stützung einer multifunktionalen Landwirtschaft und einer marktwirtschaftlichen Ausrichtung des Landwirtschaftssektors.

\section{4 Ökonomische Grundlagen der Agrarumweltpolitik}

Die Agrarumweltpolitik hat in den letzten Jahren laufend an Bedeutung gewonnen. Das hatte ursprünglich mit der Kenntnisnahme von Umweltskandalen und der Sensibilisierung der Bevölkerung für die Umweltprobleme der Landwirtschaft zu tun. Seit diesen Anfängen in den 1970er- und 1980er-Jahren hat die wissenschaftliche Evidenz dazu geführt, dass das Zusammenwirken von Nahrungsmittelproduktion und einer nachhaltigen Nutzung der natürlichen Ressourcen zu einem Kernelement der Steuerung des Landwirtschaftssektors weltweit wurde (Lichtenberg, 2002). Im Kern geht es darum, die Externalitäten, die mit der landwirtschaftlichen Produktion verbunden sind, in das Entscheidungsverhalten der Akteure der Nahrungsmittelwertschöpfungskette zu integrieren und damit eine nachhaltige Produktion zu ermöglichen (Heal und Small, 2002).

Das agrarökonomische Kernargument in der Umweltpolitik ist, dass der Schlüssel zur nachhaltigen landwirtschaftlichen Produktion nicht in der Vermeidung sämtlicher Umweltbelastungen liegt, sondern im Versuch, eine «optimale» Umweltbelastung anzustreben (Harris und Roach, 2013). Als optimal wird aus einer agrarökonomischen Perspektive der Schnittpunkt zwischen den zusätzlichen Schadens- und Vermeidungskostenfunktionen betrachtet (Rieder und Anwander Phan-huy, 1994).

Eine Schadensfunktion beschreibt den Zusammenhang zwischen der Umweltbelastung und deren gesamtgesellschaftlichen Kosten (siehe auch Box 4). Diese verläuft üblicherweise ansteigend, d.h., die Kosten nehmen mit zunehmender Umweltbelastung überproportional zu. 


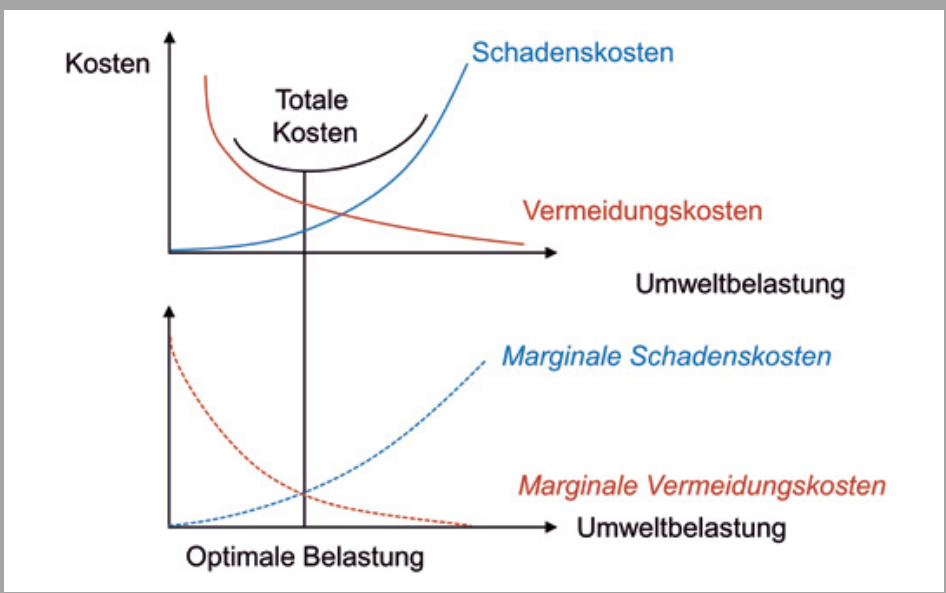

Mit der Produktion von landwirtschaftlichen Gütern ist in vielen Fällen eine Belastung der Umwelt verbunden wie beispielsweise Nitratauswaschung, Bodenerosion oder Klimagasausstoss. Diese Umweltbelastungen verursachen Schadenskosten. In den meisten Fällen nimmt dabei die Belastung überproportional zu. Dies wird durch den konvexen Verlauf der Schadenskostenkurve im oberen Panel dargestellt. Unter dieser Annahme nehmen auch die marginalen Schadenskosten, d.h. die Steigung der totalen Schadenskosten, zu (unteres Panel in der Abbildung). Mit der Reduktion dieser Umweltbelastung sind sogenannte Vermeidungskosten verbunden. Dies können z.B. Opportunitätskosten der landwirtschaftlichen Produktion sein oder konkrete landwirtschaftliche Produktionsmassnahmen, welche direkte Kosten verursachen. Diese Kosten der Vermeidung sind gering, wenn das Schadensniveau hoch ist, und nehmen zu, je mehr die Umweltbelastung vermieden werden soll. Theoretisch lässt sich auch hier eine konvexe Funktion (oberes Panel) und die Steigung dieser Kurve, d.h. die marginalen Vermeidungskosten (unteres Panel) zeichnen. Theoretisch folgt aus der Abbildung eine «optimale Umweltbelastung», nämlich dort, wo die zusätzlichen Schadenskosten genau gleich hoch sind wie die zusätzlichen Vermeidungskosten. Dies widerspiegelt, dass das ökonomische Optimum dort liegt, wo Grenzkosten und Grenznutzen gleich hoch sind. Aus dieser Perspektive ist es ökonomisch nicht sinnvoll, die Umweltbelastung vollständig zu reduzieren, weil Wohlfahrt verloren geht, wenn die marginalen Vermeidungskosten höher sind als die marginalen Schadenskosten. Es gilt zu beachten, dass dieses Optimum nicht zwangsläufig im Minimum der totalen Kosten erfolgt. 
Die Vermeidungskostenfunktion beschreibt diejenigen Kosten, die entstehen, wenn die Umweltbelastung reduziert bzw. vermieden wird. Diese nimmt üblicherweise mit zunehmender Umweltbelastung ab. Die Logik ist, dass bei hoher Umweltbelastung bereits kostengünstige Massnahmen zu einer Reduktion der Umweltbelastung führen. Je tiefer jedoch die Umweltbelastung ist (je sauberer die Luft oder das Wasser), desto teurer wird die Reduktion der Umweltbelastung. Die Idee der optimalen Umweltbelastung ist nun, dass man die beiden Kostenfunktionen zusammenzählt und das Minimum dieser totalen Kosten bestimmt. Das Minimum befindet sich dort, wo die marginalen Schadens- und Vermeidungskostenkurven sich schneiden, d.h., wo die Kosten für die Vermeidung einer zusätzlichen Einheit an Umweltbelastung exakt den zusätzlichen Kosten der nächsten Einheit an Umweltbelastung entsprechen. Dieses Konzept impliziert, dass das Ziel der Agrarumweltpolitik nicht sein kann, die Umweltbelastung gänzlich auf null zu reduzieren, sondern mit den Agrarumweltmassnahmen möglichst nahe an dieses Optimum zu kommen. Dieses Konzept ist gemeinhin anerkannt und wird auch so in der Agrarpolitik angewandt. Es gilt aber klarzustellen, dass diese agrarökonomische Betrachtung eindeutige Grenzen hat:

- Das Konzept eignet sich nicht für die konzeptionelle Herleitung von Politikmassnahmen, wenn katastrophale Auswirkungen oder Systemverschiebungen erwartet werden können (Fisher et al., 2008). Falls beispielsweise die optimale Umweltbelastung mit Treibhausgasemissionen so hoch wäre, dass die Erde unbewohnbar würde, macht die Herleitung eines Optimums wenig Sinn. Ähnliche Argumente können auch in Bezug auf die Biodiversität gemacht werden (Dasgupta, 2021).

- $\quad$ Die monetäre Bewertung der Umweltbelastung und deren Vermeidungskosten ist nicht trivial, insbesondere wenn Umweltsystemleistungen mit öffentlichem Charakter involviert sind (Perman et al., 2003; Harris und Roach, 2013). Auch hier ist die Klimadebatte ein einleuchtendes Beispiel, in welchem die Festlegung der Diskontrate (d.h. wie viel uns das jetzige Geld in der Zukunft wert ist) zu völlig unterschiedlichen Positionen in Bezug auf die optimale Belastung mit Treibhausgasen führt. Für Problemstellungen, in denen die Bewertung von Kosten mit hohen Unsicherheiten verbunden sind, ist daher eine "Optimierung» der Umweltbelastung in der Praxis schwierig umzusetzen (Dasgupta, 2021).

Grundsätzlich ist die «optimale Umweltbelastung» eine Anwendung des Prinzips, wonach eine effiziente Produktion dort stattfinden soll, wo Grenzkosten und Grenzerlöse gleich hoch sind. Mit Blick auf die Umweltbelastung wird diese analog so interpretiert, dass eine effiziente Steuerung dazu führt, dass die Kosten einer zusätzlichen Einheit Umweltbelastung gleich hoch sein sollten wie die zusätzlichen Kosten derer Vermeidung. Umgekehrt bedeutet dies, dass positive Externalitäten so weit 
ausgedehnt werden sollen, bis die zusätzlichen Kosten der Erbringung, z.B. von Biodiversitätsleistungen, gleich hoch sind wie der zusätzliche Nutzen aus der entsprechenden Umweltleistung.

Eine weitere wichtige Grundlage für das Verständnis der Agrarumweltpolitik ist die Steuerung der landwirtschaftlichen Intensität. Die Intensität beschreibt das Verhältnis zwischen zwei Produktionsfaktoren. Es ist wichtig, die Intensität von der Produktivität zu unterscheiden. Produktivität beschreibt das Verhältnis zwischen Output und Input und charakterisiert dadurch die Effizienz eines Produktionssystems. Die Intensität dagegen beschreibt die Art der Produktion. Wichtig ist auch zu bemerken, dass beide Indikatoren keine Aussagen zur Rentabilität der landwirtschaftlichen Produktion machen. Dazu eignen sich Verhältniszahlen nicht. Für eine Beurteilung der Rentabilität braucht es die Berücksichtigung aller Produktionsfaktoren und deren monetarisiertes Verhältnis zum Erlös oder Gewinn eines landwirtschaftlichen Betriebs.

Grundsätzlich gibt es in der Landwirtschaft zwei zentrale Typen von Intensitäten. Das Verhältnis zwischen Kapital und Arbeit zeigt, wie kapitalintensiv oder mechanisiert die landwirtschaftliche Produktion ist. Das Verhältnis zwischen Kapital und Boden beschreibt den Einsatz von Hilfsstoffen pro Fläche und beschreibt dadurch auch mögliche ökologische Probleme, z.B. durch Überdüngung. In der Agrarumweltpolitik ist die Steuerung der Intensität daher ein wichtiger Hebel, um die ökologischen Probleme der landwirtschaftlichen Produktion anzugehen. Die grundlegende Idee ist, dass durch eine Reduktion des Kapitaleinsatzes pro Flächeneinheit weniger Hilfsstoffe eingesetzt werden und dadurch negative Externalitäten reduziert werden können (siehe Box 5) oder positive Externalitäten wie beispielsweise Biodiversität gefördert werden. Das ist auch die grundlegende Idee hinter einer Steuer (siehe dazu das Kapitel 6.3). Je teurer der Einsatz von Hilfsstoffen ist, desto weniger wird sie ein gewinnmaximierender Landwirt einsetzen. Umgekehrt kann auch eine Senkung des OutputPreises den gleichen Effekt haben. Durch die Reduktion des Wertgrenzprodukts sinkt der Anreiz, die im Verhältnis teurer gewordenen Hilfsstoffe einzusetzen.

Es ist aber wichtig, dieses Konzept der Reduktion der «allgemeinen» Intensität einzuordnen (Rieder und Anwander Phan-huy, 1994). Der Effekt bezieht sich auf ein Verhältnis zwischen den Inputfaktoren und nicht auf den gesamten Betrieb oder eine Region. Da die Intensität nichts über die Rentabilität aussagt, ist es denkbar, dass im Optimum die landwirtschaftliche Produktion gar nicht mehr rentabel ist. 


\section{Box 5 Steuerung der Intensität}

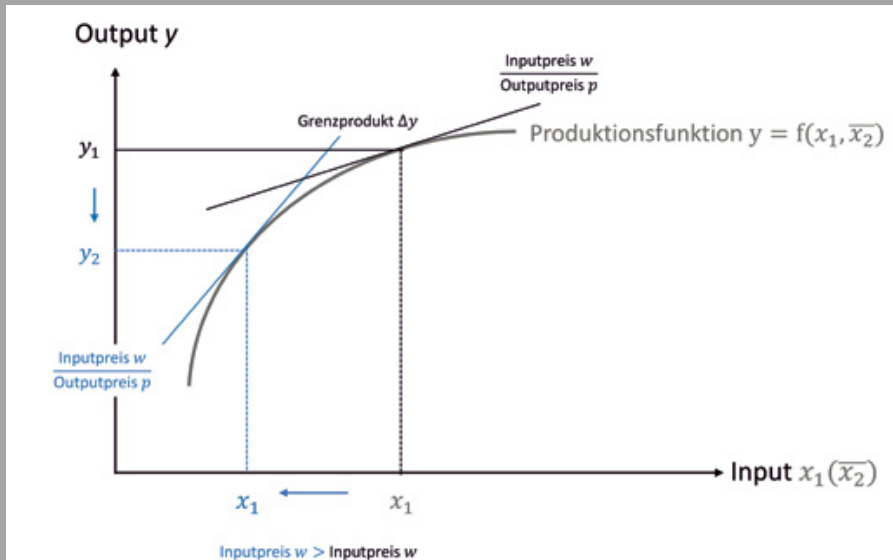

Wertgrenzprodukt $=$ Faktorpreis $(\Delta y * p=w)$

Landwirtschaftliche Produktionsfunktionen haben üblicherweise einen konkaven Verlauf. Das heisst, durch die Erhöhung der Intensität, beispielsweise durch den Einsatz von Hilfsstoffen $\left(x_{1}\right)$ wie Dünger oder Pflanzenschutzmittel pro Flächeneinheit, nimmt der Ertrag zuerst überproportional zu und nimmt in der Folge kontinuierlich ab, bis eine zusätzliche Einheit des Inputfaktors keinen zusätzlichen Ertrag mehr erbringt. In der Abbildung ist die Intensität dadurch dargestellt, dass die Fläche, der zweite Inputfaktor, konstant gehalten wird $\left(\overline{x_{2}}\right)$. Ein Landwirt, der seinen Gewinn maximieren möchte, wird die Intensität so wählen, dass der Wert des Grenzprodukts, d.h. der Erlös der nächsten Einheit des physischen Outputs $\Delta y$, gerade dem Preis für den Input entspricht (d.h. dem Faktorpreis). Wären die Kosten für eine zusätzliche Einheit des Inputfaktors $x_{1}$ höher als der zusätzliche Erlös $(\Delta y * p)$, dann würde es sich für die Bäuerin oder den Bauern finanziell nicht mehr lohnen, die Inputmenge bzw. die Intensität zu erhöhen. Dies entspricht der ökonomischen Regel, dass im Optimum der Grenzerlös und die Grenzkosten gleich hoch sein müssen. Grafisch ist dieses Optimum dadurch gekennzeichnet, dass die Steigung der Produktionsfunktion $\Delta y$ der Steigung des Preisverhältnisses von Input- zu Output-Preisen entspricht. Wird nun der Preis für den Input erhöht oder der Output-Preis gesenkt (blaue Tangente), dann wird die Gerade aus den Preisverhältnissen steiler. Die Tangente dreht sich dadurch ins neue Optimum nach links und die Produktionsintensität sowie der Ertrag gehen zurück. 
Die Fläche würde daher anders genutzt, z.B. mit einer Kultur, die höhere Erträge erbringt und damit höhere Inputpreise kompensiert. In der Agrarökonomie unterscheidet man diesbezüglich zwei Effekte. Der sogenannte «intensive margin» Effekt beschreibt, wie sich die Flächen- oder Arbeitsintensität verändert, wenn sich Preise verändern oder politische Anreize geschaffen werden (Goetz et al., 2006). Der «extensive margin» Effekt beschreibt die Veränderung der aggregierten Landnutzungsveränderungen durch die Verschiebung von Preisen und Politikmassnahmen (Huber et al., 2017b). Die Steuerung der landwirtschaftlichen Produktion über die Intensität muss immer beide Effekte berücksichtigen. Ansonsten können ungewollte Nebeneffekte entstehen. Ein Beispiel wäre, dass durch die Senkung des Mineraldüngereinsatzes im Ackerbau Grasland umgebrochen wird und die gesamte Ackerfläche ausgedehnt wird. Möglicherweise kann der Effekt, dass pro Fläche weniger Dünger eingesetzt wird (intensive margin Effekt), dadurch kompensiert werden, dass dies auf zusätzlichen Flächen geschieht (extensive margin Effekt), die vorher einen tieferen Einsatz von Mineraldüngern aufwiesen. Die Steuerung von Intensitäten ist daher eine komplexe Herausforderung und benötigt zwingend eine ganzheitliche Perspektive, um Marktversagen zu verhindern.

\subsection{Beurteilung von Agrarpolitik: Wirksamkeit und Effizienz}

Eine zentrale Herausforderung für die Agrarpolitik ist, dass durch die Stützung einer multifunktionalen Landwirtschaft Marktverzerrungen entstehen können, welche die Effektivität, aber auch die Effizienz der Massnahmen beeinträchtigen und damit zu einem Politikversagen führen können. Im Kontext der Agrarpolitik sind vor allem zwei Ursachen zentral: fehlende Wirksamkeit und Mitnahmeeffekte (van Tongeren, 2008).

Mit Blick auf die Wirksamkeit von agrarpolitischen Massnahmen hat sich im Kontext der Agrarpolitik der Begriff des Targetings (Zielausrichtung) etabliert. Targeting bedeutet, dass eine agrarpolitische Intervention zielgenau sein sollte. Sie soll also möglichst direkt auf ein einzelnes Ziel, die Erbringung einer öffentlichen Leistung oder die Reduktion einer negativen Externalität ausgerichtet sein. Das Targeting kann einen räumlichen oder einen zeitlichen Bezug haben. Das heisst, ein Ziel kann nur auf eine bestimmte Region oder eine bestimmte Zeitperiode ausgerichtet sein. Die Zielorientierung folgt einerseits aus dem Konzept der Tinbergen-Regel - jedes Ziel braucht eine (und genau) eine Massnahme. Andererseits garantiert das Targeting auch die klare Definition und damit verbunden die Messbarkeit von agrarpolitischen Zielen (siehe Politikzyklus nächstes Kapitel). Die Messbarkeit erlaubt es zu evaluieren, ob eine agrarpolitische Massnahme überhaupt einen zusätzlichen Beitrag zur Erbringung einer öffentlichen Leistung erzeugt. Dies wird als Additivität einer Massnahme bezeichnet (Wunder et al., 2018). Eine unklare Formulierung des Targetings kann dazu führen, dass agrarpolitische Massnahmen keine Wirkung, unbeabsichtigte Ne- 
beneffekte oder unerwartete Auswirkungen auf Dritte haben. Aus einer rein wohlfahrtsökonomischen Perspektive ist aber Targeting nur so lange sinnvoll, wie der positive Effekt, d.h. die gewonnene Konsumenten- oder Produzentenrente, grösser ist als die damit verbundenen Transaktionskosten, d.h. die administrativen Kosten aufseiten des Bundes und der Akteure in der Wertschöpfungskette (Vatn, 2002).

Mit Blick auf die klassischen Mitnahmeeffekte von agrarpolitischen Massnahmen wird aus einer rationalen Perspektive das sogenannte Tailoring angestrebt. Dieses Prinzip besagt, dass die agrarpolitischen Massnahmen auf diejenigen Betriebe ausgerichtet werden sollen, welche die Leistung auch tatsächlich erbringen. Auch das Tailoring kann räumlich oder zeitlich begrenzt werden. Dadurch werden Mitnahmeeffekte und "Rent-Seeking» verhindert (OECD, 2003; van Tongeren, 2008). Diesem Prinzip folgend ist es beispielsweise sinnlos, die Offenhaltung von landwirtschaftlichen Flächen in Gebieten zu fördern, die gar nicht von einer Einwaldung bedroht sind. Es ist aus einer rationalen Perspektive ebenfalls fragwürdig, Biodiversitätsförderflächen mit Versorgungssicherheitsbeiträgen zu unterstützen.

Das klassische Beispiel für ein Tailoring in der Schweizer Agrarpolitik sind die Beiträge für die Biodiversitätsförderflächen. Diese sind nach Zonen abgestuft, d.h. zugeschnitten oder eben «tailored», weil sich die Opportunitätskosten einer extensiven Bewirtschaftung in den einzelnen Höhenstufen stark voneinander unterscheiden. Das Tailoring spielt aber nicht nur auf der Ebene von Direktzahlungen, sondern für fast alle agrarpolitischen Massnahmen eine Rolle. Die Logik ist, dass Massnahmen, die undifferenziert sind (One Size Fits All), die Heterogenität der Landwirtschaftsbetriebe in der Schweiz nicht berücksichtigen und daher automatisch zu Mitnahmeeffekten führen. Das klassische Beispiel ist hier der Zoll. Ohne Differenzierung, z.B. über Zollkontingente oder verschiedene Produktkategorien, wirkt sich eine Erhöhung des Inlandpreises für alle Betriebe gleich aus, unabhängig davon, ob er finanziell auf eine Preiserhöhung angewiesen ist oder nicht.

Tailoring hat aber auch Nachteile: 1) Mit einem Zuschneiden der Massnahmen stellt sich immer auch die Frage nach der Verteilungswirkung der Massnahme, z.B. Unterschiedliche Zahlungen an Berg- und Talbetriebe. Theoretisch lässt sich das Problem von unterschiedlichen Kostenstrukturen über einen Markt für öffentliche Güter lösen, z.B. eine Versteigerung (Höchli et al., 2009). Dadurch lassen sich mögliche wohlfahrtstheoretische Gewinne im agrarpolitischen Prozess aber nicht immer rechtfertigen und durchsetzen. Ausserdem zeigen Untersuchungen im Kontext von Zahlungen für Umweltleistungen, dass eine ungleiche Verteilung, eben durch das Tailoring, dazu führt, dass die Landwirte die Zahlung als ungerecht empfinden. Die benachteiligten Betriebe bieten dadurch bedeutend weniger Umweltleistungen an, was insgesamt zu einem negativen Effekt führen kann (Loft et al., 2020). 

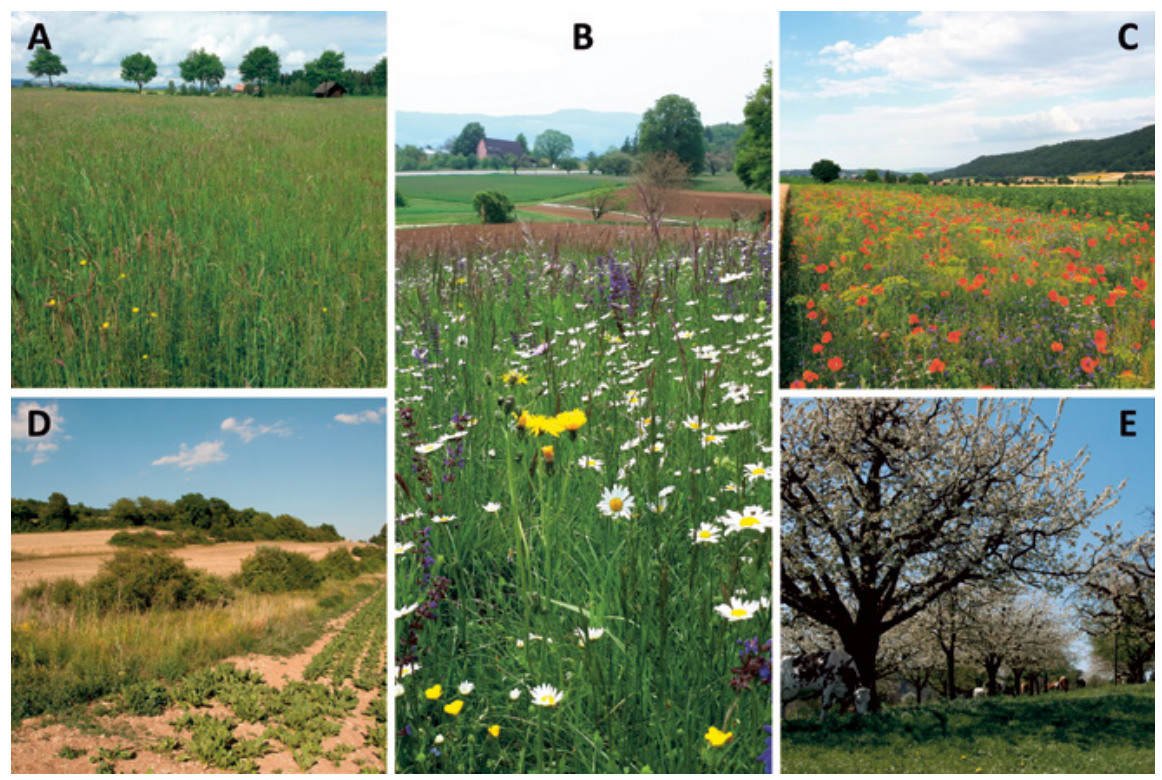

Abbildung 4. Die Biodiversitätsbeiträge in der Schweiz haben unterschiedliche Ziele (Erhaltung seltener Arten, Förderung der Vernetzung von naturnahen Lebensräumen) und sind auf unterschiedliche Massnahmen in den landwirtschaftlichen Zonen zugeschnitten. Sie sind damit ein typisches Beispiel für das Targeting und Tailoring von agrarpolitischen Massnahmen. A: artenarme Extensivwiese (BFFQI); B: artenreiche Extensivwiese mit hoher biologischer Qualität (BFFQII); C: Nützlingsblühstreifen im Ackerland (BFFQI); D: Gebüschgruppen und Baumhecken vernetzen und gliedern die Agrarlandschaft; E: Hochstammobstbäume als vertikale Elemente der Biodiversitätsförderung. (Bilder: Roman Graf \& Markus Jenny, Vogelwarte Sempach, und Katja Jacot, Agroscope)

Wirksamkeit und Effizienz einer agrarpolitischen Massnahme müssen daher immer auch mit Blick auf die Verteilungswirkung beurteilt werden. 2) Das Zuschneiden führt dazu, dass die Steuergelder (Direktzahlungen) effizienter eingesetzt werden können. Die Landwirte verlieren aber einen Teil der Produzentenrente. Während in einem realen Markt auch Gewinne gemacht werden können, die über den Herstellungskosten liegen, z.B. über Produktdifferenzierungen oder Marken, werden die Landwirte in diesem System ausschliesslich für ihre Kosten entschädigt (WTO: Foregone income). Es stellt sich daher die Frage, wieso Betriebe mit einer günstigen Kostenstruktur mit Direktzahlungen nicht auch Gewinne erzielen dürfen (Mann 2006a). Die WTO sagt, aus Effizienzgründen, nein (Matthews, 2013) - die Verteilungswirkung von Direktzahlungen ist dennoch ein wichtiger Grund für viele Subventionen im Agrarbereich. In der Schweiz sorgen beispielsweise die Versorgungssicherheitszahlungen für einen erheblichen Einkommenstransfer (Mann und Mack, 2004; Möhring et al., 2018). 3) Auch im Kontext des Tailoring stehen den wohlfahrtstheoretischen Gewinnen die administrativen Transaktionskosten entgegen. 
Targeting und Tailoring werden oft in einem Zug genannt (van Tongeren, 2008). Es werden aber unterschiedliche Herausforderungen in der Umsetzung von agrarpolitischen Massnahmen angegangen. Dabei ist zu beachten, dass die Koppelproduktion in der Landwirtschaft wiederum dazu führt, dass Targeting und Tailoring sich gegenseitig beeinflussen können und kein Allerheilmittel sind. Als Beispiel seien hier die Landschaftsqualitätsbeiträge genannt. Diese projektbezogenen Zahlungen sind spezifisch auf ausgewählte Betriebe zugeschnitten (tailored). Um dies sicherzustellen, sind auch die Ziele in den Projektgebieten individuell festgelegt (targeted). Damit verbunden sind, relativ zu anderen Direktzahlungen, hohe administrative Aufwände und Transaktionskosten (Planung, Monitoring, Kontrolle). Die Evaluation des Instruments hat aber gezeigt, dass die Landwirte hauptsächlich diejenigen Massnahmen anmelden, die sie schon vorher auf ihren Betrieben umgesetzt hatten (Mitnahmeeffekte). Inwiefern das Instrument langfristig zur Erhaltung der Landschaftsqualität beitragen kann, ist daher offen.

\section{ZUSAMMENFASSUNG}

Die Agrarpolitik orientiert sich immer an einem Zielsystem. Die Anzahl der Ziele, die mit der Agrarpolitik erreicht werden sollen, hat im Verlauf der letzten Jahrzehnte zugenommen. Auch die Gewichtung der Ziele obliegt einer ständigen Veränderung. Aus einer theoretischen Perspektive wäre es optimal, wenn es für jedes agrarpolitische Ziel auch ein entsprechendes Instrument gäbe (Tinbergen-Regel). Auf diese Weise könnten die Ziele auf eine effiziente Weise erreicht werden. Eine zentrale Herausforderung der Agrarpolitik ist aber, dass die unterschiedlichen ökonomischen, ökologischen und sozialen Ziele sich gegenseitig beeinflussen oder sogar miteinander in Konflikt stehen können. Aufgrund der komplexen natürlichen und sozioökonomischen Interaktionen der Landwirtschaft lassen sich diese "Trade-offs» nur bedingt zielgerichtet ansteuern. Die Politik muss daher einerseits Güterabwägungen treffen und agrarpolitische Ziele unterschiedlich gewichten. Andererseits muss die Politik aber auch den richtigen Mix an Massnahmen finden, welcher die Ziele möglichst gut erreicht (Wirksamkeit) und gleichzeitig die limitierten finanziellen Ressourcen möglichst effizient einsetzt. Um Mitnahmeeffekte und Rent-Seeking zu verhindern, sollten die gewählten Massnahmen «targeted» und "tailored» sein. Im optimalen Fall erfüllen sie dadurch die Tinbergen-Regel und führen zu einer effektiven und effizienten Agrarpolitik. Eine wichtige (ordnungspolitische) Rechtfertigung für ein politisches Eingreifen in die Landwirtschaft ist, dass ein Marktversagen vorliegt. Im Kontext der Landwirtschaft stehen dabei drei Aspekte im Vordergrund: Externalitäten, unvollständiger Wettbewerb und Informationsasymmetrien. Aus einer wohlfahrtsökonomischen Perspektive ist Marktversagen aber lediglich eine notwendige Bedingung für agrarpolitische Interventionen. Darüber hinaus müssen die eingeführten Massnahmen aber auch zu einer wohlfahrtsökonomischen Verbesserung der Ressourcenallokation führen. Ansonsten können agrarpolitische Eingriffe zu Politikversagen führen. 
Das Zusammenspiel zwischen Akteuren, Märkten und staatlichen Eingriffen wird dabei als Marktordnung bezeichnet. Aufbauend auf diesen Überlegungen wird in der europäischen und Schweizer Landwirtschaft die Multifunktionalität der Landwirtschaft als zentrale Rechtfertigung für staatliche Eingriffe herangezogen. Die agrarökonomischen Grundlagen der Multifunktionalität sind die Koppelproduktion und Marktversagen. Der Schlüsselmechanismus der Agrarpolitik zur Unterstützung der Multifunktionalität ist die Entkopplung (von Preisen, Einkommen und der Bereitstellung öffentlicher Güter). Schliesslich ist im Kontext der Agrarumweltpolitik der Begriff der optimalen Umweltbelastung wichtig. Dieser impliziert, dass es auch im Bereich der Umweltmassnahmen ökonomisch nicht sinnvoll ist, die negativen Externalitäten der landwirtschaftlichen Produktion auf null zu senken, weil damit sehr hohe Vermeidungskosten verbunden sind.

\section{WEITERFÜHRENDE LITERATUR}

Das Thema der Multifunktionalität ist in den letzten Jahren etwas aus dem Fokus geraten. Ein immer noch aktuelles und ganzheitliches Buch bleibt aber das Werk von Geoff A. Wilson mit dem Titel Multifunctional Agriculture: A Transition Theory Perspective.

Die Beiträge in der Zeitschrift Nature Ecology \& Evaluation von David Gawith und lan Hodge mit dem Titel «Focus rural land policies on ecosystem services, not agricuIture» sowie Pete Manning und Koautoren mit dem Titel «Redefining ecosystem multifunctionality» werfen einen ökologischen Blick auf die Multifunktionalität der Landwirtschaft.

Ressourcenökonomische Grundlagen, inlusive Markt- und Politikversagen, werden beispielsweise im Lehrbuch von Perman und Koautoren Natural resource and environmental economics ausführlich und verständlich beschrieben.

Wie Wertschöpfungsketten im Bereich der Land- und Ernährungswirtschaft funktionieren und welche Herausforderungen sich daraus ergeben, wird im Artikel von Richard Sexton «Market Power, Misconceptions, and Modern Agricultural Markets» gut beschrieben. 


\section{Instrumente der Agrarpolitik (Policy)}

\section{READERS' GUIDE}

In diesem Kapitel werden die konzeptionellen und wohlfahrtstheoretischen Grundlagen von agrarpolitischen Instrumenten behandelt. Das Kapitel besteht aus drei Teilen. In einem ersten Unterkapitel werden aus einer theoretischen Perspektive acht Dimensionen von agrarpolitischen Instrumenten erörtert. Dabei geht es darum zu definieren, wie die Instrumente überhaupt ausgestaltet werden können. Man spricht in diesem Zusammenhang auch vom «Design» oder der Ausgestaltung von Politikmassnahmen. Diese Dimensionen sind nachher wichtig, um die tatsächlich angewendeten Instrumente einordnen zu können und mögliche alternative Ausgestaltungen zu kennen. In einem zweiten Teil werden die Grundbegriffe für die Evaluation von agrarpolitischen Massnahmen dargelegt. Dabei geht es um die Beurteilungskriterien Relevanz, Effektivität und Effizienz und das sogenannte Wirkungsmodell, mit welchem Politikmassnahmen evaluiert werden. Zusammen mit dem Verständnis über die verschiedenen Dimensionen legt dieses Kapitel legt den Grundstein für die Beurteilung der aktuell angewendeten agrarpolitischen Instrumente. Im dritten Teil dieses Kapitels werden schliesslich spezifische Kategorien von Instrumenten vorgestellt. Insgesamt umfasst dieser dritte Teil sechs Kapitel. In den ersten beiden Kapiteln werden die sogenannten Command and Control und die Informationsinstrumente vorgestellt. In den Kapiteln drei bis sechs werden anschliessend die ökonomischen Instrumente mithilfe des wohlfahrtsökonomischen Rentenansatzes auf ihre theoretische Wirkung hin beurteilt. Dabei werden Instrumente des Aussenhandels, der Steuerung von Angebot und Nachfrage, agrarumweltpolitische Massnahmen sowie einkommenspolitische Massnahmen beschrieben. Das Ziel dieses Kapitels ist es, dass die Leserinnen und Leser die wichtigsten agrarpolitischen Instrumente kennenlernen, deren Wirkung beurteilen und alternative Ausgestaltungen dieser Instrumente erörtern können. 


\subsection{Dimensionen von Agrarpolitikmassnahmen}

Als Folge der unterschiedlichen ökonomischen, ökologischen und sozialen Ziele der Agrarpolitik existiert auch eine vielfältige Palette von agrarpolitischen Instrumenten und Massnahmen. ${ }^{7}$ Einerseits lassen sich die agrarpolitischen Instrumente in drei grundsätzliche Kategorien einteilen: 1) Command and Control, 2) Information und 3) ökonomische Anreize wie Steuern oder Subventionen (Sterner et al., 2019). Andererseits lassen sich die agrarpolitischen Instrumente auch nach ihrem Wirkungsbereich unterscheiden wie beispielsweise Einkommenspolitik, Agrarumweltpolitik oder Agrarhandelspolitik. Im dritten Teil dieses Kapitels werden die entsprechenden Kategorien ausführlich beschrieben.

Bevor wir den Blick auf die einzelnen Instrumente richten, ist es jedoch wichtig, festzuhalten, dass die Wirkung immer auch von der spezifischen Ausgestaltung einer Massnahme abhängt. «Der Teufel steckt im Detail», wie es eine der führenden Wissenschaftlerinnen in der Umweltpolitik ausdrücken würde (Engel, 2016). Mit anderen Worten, eine Direktzahlung ist nicht einfach eine Subvention. Je nachdem, wie die Zahlung designt ist, kann sie ganz unterschiedlich wirken. Sie kann beispielsweise einfach die Produktion von landwirtschaftlichen Gütern subventionieren. Im Gegensatz dazu kann sie aber als Abgeltung für öffentliche Güter, z.B. die Erhaltung von Biodiversität, ausgestaltet werden. Auch wenn beides grundsätzlich als Direktzahlung beschrieben werden kann, so besteht doch ein himmelweiter Unterschied in der Wirkung der entsprechend ausgestalteten Massnahme.

Der Punkt ist, dass ein agrarpolitisches Ziel nicht nur mit ganz unterschiedlichen Instrumenten anvisiert werden kann. Das Ziel der Versorgungssicherheit kann z.B. über Zölle, Direktzahlungen, Investitionshilfen oder Versicherungen geregelt werden. Sondern dass es auch möglich ist, innerhalb dieser Kategorien von Instrumenten, eine agrarpolitische Massnahme ganz unterschiedlich auszugestalten. Es ist daher zentral, die grundlegenden Ausgestaltungsmöglichkeiten von agrarpolitischen Massnahmen zu kennen, weil die Art des Designs gewollte und ungewollte Effekte entlang des gesamten Ernährungssystems haben kann.

Grundsätzlich lassen sich die Ausgestaltungsmöglichkeiten in sogenannte Dimensionen unterteilen (Richards, 2000; Ehlers et al., 2021). Im Folgenden werden acht Dimensionen von agrarpolitischen Massnahmen vorgestellt, die sich auf drei unterschiedliche institutionelle und räumliche Ebenen beziehen (Abbildung 5).

- Input-Outcome-Beziehung: Eine agrarpolitische Massnahme kann sich entweder auf einen Input in die landwirtschaftliche Produktion beziehen (z.B. auf

\footnotetext{
${ }^{7}$ Agrarpolitische Massnahmen und agrarpolitische Instrumente werden in diesem Buch synonym verwendet.
} 
die genutzte Fläche oder die Menge an eingesetztem Dünger) oder auf den Outcome (wie z.B. die Biodiversität in der Agrarlandschaft oder das Einkommen der Landwirte). Wichtig ist dabei, dass Massnahmen, die sich auf einen Input beziehen, einfacher umzusetzen und zu kontrollieren sind. Je besser der Input mit dem Outcome korreliert, desto einfacher ist die Steuerung über eine inputorientierte Massnahme. Outcome-orientierte Massnahmen hingegen sind, wie es der Name schon sagt, stärker auf das eigentliche Ziel ausgerichtet und damit effektiver. Sie sind insbesondere dann wirkungsvoll, wenn der Outcome von vielen (Input-)Faktoren beeinflusst wird und daher die Korrelation zwischen Input und Outcome nicht stark ist. Ausserdem können sich outcome-orientierte Massnahmen auch auf überbetriebliche Ziele beziehen, wie beispielsweise die Erhaltung der Schönheit einer Landschaft.

- Standortabhängigkeit: Ein agrarpolitisches Instrument kann räumlich ganz explizit sein, indem es z.B. vorschreibt, auf welchem Feld eine gewisse Bewirtschaftungsweise erlaubt ist und auf welchem nicht. Im Gegensatz dazu kann auch eine Massnahme innerhalb einer bestimmten Agrarpolitik überhaupt keinen räumlichen Bezug haben. So regeln zum Beispiel Zölle den Preis für gewisse landwirtschaftliche Produkte an der Grenze. Davon profitieren sämtliche Bauern - unabhängig von ihrem Standort innerhalb der Schweiz. Der Fachbegriff für eine räumlich möglichst differenzierte Ausgestaltung von Massnahmen lautet «Spatial Targeting». Grundsätzlich erhöht die räumliche Differenzierung der Massnahmen deren Effektivität und Effizienz - insbesondere im Agrarumweltbereich (Wünscher et al., 2008) und vor allem dann, wenn die öffentlichen Mittel knapp sind und priorisiert werden müssen. Diese Massnahmen sind aber oft auch komplex in der Umsetzung.

- Intertemporale Flexibilität: Neben einer räumlichen Differenzierung ist es auch möglich, agrarpolitische Massnahmen über die Zeit flexibel zu gestalten. Das klassische Beispiel dafür sind die Zollkontingente, welche die inländische Produktion vor Importen schützen soll (siehe Kapitel zum Aussenhandel). Diese können so ausgestaltet werden, dass sie nur dann eine Wirkung erzielen, wenn im Inland tatsächlich produziert wird. Das heisst, ein effektiver, hoher Zoll gilt dann nur in einer bestimmten Jahreszeit. Im Rest des Jahres gilt dann ein tieferer Zoll. Umgekehrt können in agrarpolitischen Massnahmen auch längere Zeitperioden fixiert werden. So gelten z.B. Mindestvertragslängen für die Teilnahme an Agrarumweltprogrammen oder Rückzahlungsfristen für Investitionshilfen.

- $\quad$ Ermessensspielraum der Landwirte bzw. des Staates: Grundsätzlich lässt sich diese Dimension gut anhand der zwei Extremausprägungen erklären. Einerseits kann die Regierung eine bestimmte Regelung vorlegen, die der Landwirt zu befolgen hat. In diesem Fall liegt der Ermessensspielraum ganz beim Staat 
und der Bauer folgt der entsprechenden Regulierung. Die klassischen Beispiele sind die "Command and Control»-Instrumente (siehe nächstes Unterkapitel). Andererseits kann der Ermessensspielraum ganz auf der Seite der Bäuerin oder des Bauern liegen. Wenn der Staat z.B. vorgibt, welche seltenen Pflanzen er auf einer Wiese wünscht und er dafür eine Direktzahlung ausspricht, ohne dass er Vorgaben zur Bewirtschaftung macht, dann liegt es völlig im Ermessen des Landwirts, wie er dieses Ziel erreicht. Grundsätzlich haben Massnahmen, die es dem Landwirt erlauben, die eigenen strukturellen und betriebswirtschaftlichen Voraussetzungen zu berücksichtigen, eine höhere Akzeptanz in der Landwirtschaft. Der Nachteil ist, dass die Kontrolle meist schwierig ist und die Umsetzung oft sehr komplex wird.

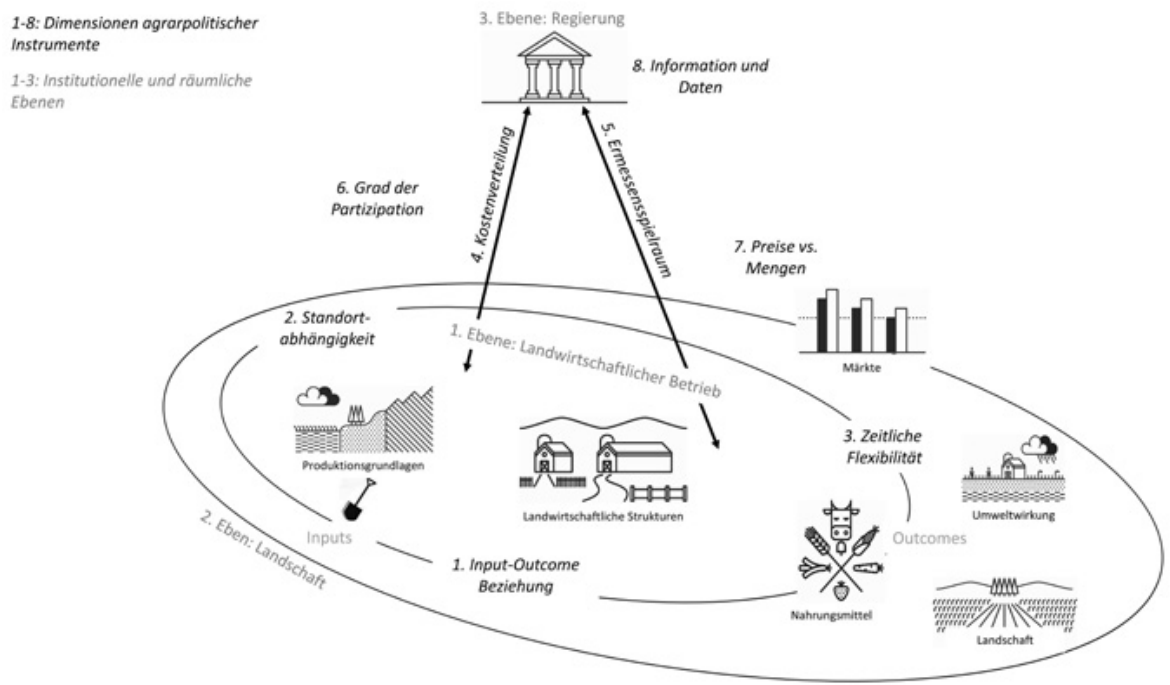

Abbildung 5. Dimensionen von agrarpolitischen Instrumenten über institutionelle und räumliche Ebenen.

- $\quad$ Verteilung der Kosten: Eine zentrale Dimension für die Ausgestaltung von agrarpolitischen Massnahmen ist, wer die Kosten einer Massnahme trägt. Im Bereich der ökonomischen Instrumente kann man dabei grundsätzlich zwischen Steuern und Subventionen unterscheiden. Wird ein landwirtschaftlicher Inputfaktor besteuert, dann trägt die Bäuerin oder der Bauer die Kosten. Umgekehrt erhält der Landwirt bei einer Subvention Geld von der Regierung. Der Steuerzahler als Finanzierer des Staates trägt in diesem Fall die Kosten. Diese Dimension hat stark mit der Definition von Eigentumsrechten zu tun. Gehört die Umwelt dem Landwirt und wird ihm dadurch das Recht auf Verschmutzung erteilt, dann müsste man ihn entschädigen, wenn er dies un- 
terlassen soll. Umgekehrt kann man die Umwelt auch als Eigentum der Gesellschaft betrachten und der Landwirt muss für die Verschmutzung bezahlen (Bromley, 2000). Darüber hinaus sollten in der Umsetzung von agrarpolitischen Massnahmen immer auch die Transaktionskosten sowohl der Behörde als auch der landwirtschaftlichen Betriebe berücksichtigt werden (Rørstad et al., 2007; Mettepenningen et al., 2011).

- $\quad$ Grad der Partizipation: Neben der Frage, wo der Ermessensspielraum liegt bei der Regierung oder dem Landwirt-, kann eine agrarpolitische Massnahme auch in Bezug auf die Partizipation unterschiedlich ausgestaltet sein. Eine Massnahme kann z.B. obligatorisch oder freiwillig sein. Das heisst, die Betriebe können selbst entscheiden, ob sie an einem Programm mitmachen wollen oder nicht. Dabei gibt es auch Mischformen. Der ökologische Leistungsnachweis, den Standard, den die Landwirte erfüllen müssen, um in der Schweiz Direktzahlungen zu erhalten, ist eine sogenannte Cross-ComplianceMassnahme. Das bedeutet, ein Betrieb erhält nur dann Direktzahlungen, wenn er diese Standards einhält. Theoretisch ist die Einhaltung des ökologischen Leistungsnachweises freiwillig. Eine Bäuerin oder ein Bauer kann auch auf die Einhaltung und damit auf die Direktzahlungen verzichten. Sie oder er müsste dann nur noch die Standards im Umwelt- und Tierschutzgesetz einhalten. Faktisch ist aber der Anreiz über die hohen Direktzahlungen so gross, dass mit wenige Ausnahmen fast alle landwirtschaftlichen Betriebe in der Schweiz den ökologischen Leistungsnachweis erfüllen. Die sozioökonomischen Rahmenbedingungen lassen die formale Freiwilligkeit gar nicht zu. Neben der Frage nach der Freiwilligkeit adressiert diese Dimension auch die Frage, inwieweit der Landwirt selbst an der Umsetzung der Massnahme partizipieren kann. Ein Beispiel dafür sind die sogenannten projektbezogenen agrarpolitischen Massnahmen. Diese erfordern, dass sich die Bäuerinnen und Bauern aktiv an der Umsetzung eines Instruments beteiligen, z.B. indem sie eigene Ziele und Massnahmen auf Landschaftsebene definieren. Beispiele dafür sind die Landschaftsqualitätsprojekte oder die Projekte regionaler Entwicklung (siehe dazu das Kapitel 9).

- $\quad$ Preis vs. Menge: Die Erreichung eines agrarpolitischen Ziels mittels ökonomischer Instrumente kann immer auf zwei Arten angegangen werden. Entweder die Politikmassnahme zielt auf die produzierte Menge oder auf den Preis eines Gutes. Ein Kontingent, zum Beispiel, legt eine genaue Menge fest. Der Preis ergibt sich dann aus dem Zusammenspiel zwischen den Marktkräften und den anderen politischen Rahmenbedingungen. Eine Politikmassnahme kann aber auch ganz gezielt den Preis festlegen, beispielsweise über einen Zoll. In diesem Fall ergibt sich dann die anvisierte Menge aus dem Zusammenspiel der Politik- und Marktkräfte. Wichtig ist, dass auch über die Festlegung 
von Mengen eine Wettbewerbssituation entstehen kann, z.B. wenn man Kontingentsanteile handeln darf oder wenn Mengen versteigert werden.

- Information und Daten: Hinter dieser Dimension stecken zwei Aspekte. Einerseits können agrarpolitische Massnahmen ganz einfach darin bestehen, Informationen und Wissen zur Verfügung zu stellen (siehe dazu auch das nächste Kapitel). Dazu gehören z.B. die Aus- und Weiterbildung oder Informationen zu Marktpreisen oder Labels. Diese Art von Massnahme unterscheidet sich prinzipiell von «Command and Control» oder ökonomischen Instrumenten. Andererseits geht es um den Austausch von Informationen oder Daten zwischen dem Landwirt und der Regierung. Dieser Austausch kann direkt zwischen Regierung (in der Schweiz dem Kanton) und dem Landwirt stattfinden. Es gibt aber auch private Dritte, die diesen Austausch sicherstellen können, z.B. Verbände (Preisinformationen der Branchenorganisation der Schweizer Fleischwirtschaft Proviande) oder Unternehmen (Tierverkehrsdatenbank der identitas). Wichtig ist in diesem Kontext, dass die Dimension der Datenhoheit für die Umsetzung von agrarpolitischen Massnahmen eine wichtige Rolle spielt und deren Umsetzbarkeit und Effektivität beeinflussen kann. Je mehr Informationen ausgetauscht werden, desto einfacher fällt es dem Regulator, die Massnahmen zu kontrollieren und evaluieren. Die Bäuerinnen und Bauern müssen dafür aber viele unternehmerische Informationen preisgeben. Dahinter verbirgt sich eine grosse Herausforderung für die Umsetzung der Agrarpolitik: Wie können agrarpolitische Massnahmen implementiert, kontrolliert und evaluiert werden, ohne den Landwirten einen grossen administrativen Aufwand aufzubürden und ohne ihren Anspruch auf Datenschutz zu stark einzuschränken.

Mithilfe dieser Dimensionen lassen sich agrarpolitische Massnahmen exakt beschreiben und deren Wirkung abschätzen. An dieser Stelle sei auch ein Rückbezug auf die Kategorien der OECD erlaubt (Kapitel 4.2). Die OECD beurteilt die agrarpolitischen Massnahmen nach deren Wirkung auf die Produktion. Die Darstellung der unterschiedlichen Dimensionen von agrarpolitischen Instrumenten zeigt, wie schwierig das ist. Eine Massnahme kann unverfänglich als Direktzahlung taxiert werden, ist aber so ausgestaltet, dass sie als reine Produktionssubvention wirkt. Die Kenntnis über die genaue Ausgestaltung von agrarpolitischen Massnahmen ist daher eine wichtige Voraussetzung für eine sinnvolle Beurteilung von Instrumenten.

Wichtig ist auch die Beziehung zwischen den Dimensionen und den Begriffen Targeting und Tailoring (aus den Grundlagen im Kapitel 5.1). Die Dimensionen «Input-Outcome-Beziehung», "Standortabhängigkeit» und "Intertemporale Flexibilität» sind die Grundlagen für die Zielausrichtung eines Instruments oder eben das Targeting. Tailoring dagegen bezieht sich in erster Linie auf die Dimensionen «Grad der Partizipation», "Verteilung der Kosten» und die «Standortabhängigkeit». Auch hier hilft die 
Kenntnis über die genaue Ausgestaltung einer agrarpolitischen Massnahme zu verstehen, was genau mit «targeted» und «tailored» im konkreten Fall gemeint ist.

In den beiden folgenden Kapiteln werden nun zuerst die Grundbegriffe der Evaluation eingeführt und anschliessend verschiedene Kategorien von Instrumenten aus einer wohlfahrtstheoretischen Perspektive betrachtet.

\subsection{Wohlfahrtsanalytische Beurteilung von Politikmassnahmen}

Im Folgenden werden sechs Kategorien von agrarpolitischen Massnahmen vertieft vorgestellt: Command and Control, Informationsmassnahmen, Aussenhandel, Steuerung von Angebot und Nachfrage im Inland, Agrarumweltpolitik sowie Einkommenspolitik. Wenn möglich, wird dabei auf eine wohlfahrtsökonomische Interpretation der Auswirkungen abgestützt, d.h. auf die Veränderung der Produzenten- und Konsumentenrente sowie die Belastung der Steuerzahler. Diese werden in einem PreisMengen-Diagramm dargestellt. Dabei gilt es, die grundlegenden Theoreme der Wohlfahrtsökonomie zu beachten.

Das erste Theorem besagt, dass jedes Wettbewerbsgleichgewicht ein Paretooptimum ist, d.h., es kann niemand bessergestellt werden, ohne dass jemand anderer schlechtergestellt wird. Es gilt aber zu beachten, dass dieses Theorem nichts über die Verteilung der Gewinne aussagt (siehe Edgeworth-Diagramm in Varian, Kapitel 29). Das zweite Theorem besagt, dass jede effiziente Allokation nach Pareto unter bestimmten Bedingungen als Wettbewerbsgleichgewicht erreicht werden kann. Ausschlaggebend dafür sind die Anfangsausstattungen der Ressourcen und Preise, die garantieren, dass alle Haushalte ihren Nutzen und alle Unternehmen ihren Gewinn maximieren. Dieses zweite Wohlfahrtstheorem impliziert, dass die Probleme der Verteilung und der Effizienz getrennt werden können. Mit anderen Worten: Um ein Paretooptimum zu erreichen, das als gerecht betrachtet wird, muss man nicht den Wettbewerb abschaffen, sondern die Ressourcen (die Anfangsausstattung mit Gütern/Einkommen) anpassen. Für die nachfolgenden Ausführungen gilt es daher zu beachten, dass

- die Grundannahme hinter den Angebots- und Nachfragediagrammen die Effizienz der Märkte und die dahinterliegenden Annahmen (homogene Güter, vollständige Information, atomistische Marktstruktur, rationale Entscheidungen) sind. Je nach realer Ausgestaltung des Marktes müssen erweiterte Modelle berücksichtigt werden.

- die meisten der besprochenen Effekte statischer Natur sind. Die zeitliche und räumliche Dynamik der Instrumente kann zu einer differenzierten Beurteilung der wohlfahrtstheoretischen Effekte führen. Für eine gesamtheitliche Beurteilung sind daher komplexere Erklärungsmodelle notwendig. 
- $\quad$ die Auswirkungen der Instrumente zwangsläufig abhängig von den Elastizitäten der Nachfrage und des Angebots sind. Je nachdem, wie steil oder wie flach man Angebot und Nachfrage zeichnet, desto grösser oder kleiner kann ein Effekt sein. Die Effekte müssen daher immer empirisch überprüft werden.

- $\quad$ die verschiedenen Instrumente interagieren. Eine Entflechtung der Wirkung ist daher nicht immer zweifelsfrei möglich. Oft fehlen auch Daten, um die Wirkung effektiv zu bestimmen, z.B. Kosten in der Verarbeitung von Nahrungsmitteln.

Schliesslich muss beachtet werden, dass die Trennung von Allokations- und Verteilungseffekten, welche ja auch das Gerüst für das Decoupling liefert, oft mehr als nur Umverteilung, sondern auch Sicherheits- oder Gesundheitsaspekte beinhaltet. In einer agrarökonomischen Zusammenfassung der Literatur zu Kosten und Nutzen des Agrarhandels schreiben z.B. Salvatici und Nenci (2017), dass es am Ende der Bevölkerung nicht darum geht, dass irgendeine monetäre Kompensation stattfindet, sondern darum, dass die Leute eine Arbeit haben, angemessene Löhne erhalten, sicher sind, eine Gesundheitsversorgung erhalten und sich ernähren können. Dies führt uns zurück zum Zielsystem der Agrarpolitik und verbindet es mit den politischen Prozessen, die im Kapitel 11 ausgeführt werden. An dieser Stelle bleibt festzuhalten, dass die wohlfahrtstheoretische Analyse ein wichtiges Instrument zur Beurteilung von agrarpolitischen Instrumenten ist, aber allein keine ganzheitliche Perspektive auf die Agrarpolitik bieten kann.

\subsubsection{Command-and-Control-Instrumente}

«Command and Control»-Massnahmen sind grundsätzlich ein einfaches Werkzeug der Agrarpolitik. Die regulierende Behörde verbietet eine Produktionsmethode (Hühner in Käfigen) oder setzt einen Grenzwert fest (z.B. für das Ausbringen von Pflanzenschutzmitteln oder den Einsatz von Dünger) und kontrolliert diesen. Die Landwirte werden bestraft, wenn sie gegen die entsprechende Vorschrift verstossen. Die rechtliche Grundlage für diese Massnahmen sind nicht immer im LwG verankert, sondern auch im Gewässerschutzgesetz oder im Tierschutzgesetz. Es findet daher auch keine Kompensation der Landwirte statt, weil die Eigentumsrechte einer intakten Umwelt bei den Bürgerinnen und Bürgern höher gewichtet werden als die Wirtschaftsfreiheit der Landwirte. Die Bestimmung dieser Grenzen ist das Resultat ständiger politischer Verhandlungen. Dabei birgt grosse Herausforderungen, dass es sich bei vielen relevanten Stoffen in der Umwelt, die aus der Landwirtschaft stammen, um sogenannte "Non-Point Source Pollution» handelt (Shortle und Horan, 2001). Das bedeutet, dass die Quelle der Verschmutzung nicht einwandfrei festgestellt werden kann. Dies kann das Monitoring und damit die Kontrolle erschweren. 


\subsubsection{Information}

Aus einer wohlfahrtsökonomischen Perspektive sind die Schaffung von Eigentumsrechten, d.h. die Definition der Ressourcenausstattung, oder die Stärkung von Verhandlungslösungen innerhalb des Landwirtschaftssektors wichtige agrarpolitische Instrumente (Hendrikse, 2003). Im Kontext der Schweizer Agrarpolitik sind diese Aspekte jedoch vorwiegend gegeben. In weniger entwickelten Regionen der Welt ist jedoch die Festlegung oder zumindest die Möglichkeit der Festlegung der Eigentumsrechte ein zentrales Instrument für die Landwirtschaftspolitik (Deininger und Feder, 2009). Dazu gehört auch, dass die entsprechenden Rechte transparent sind, um Korruption zu verhindern (Lawry et al., 2014).

Zudem kann der Staat Informationen und Wissen zur Verfügung stellen, welche Bäuerinnen und Bauern zu bestimmten Verhaltensweisen angehalten werden, und die Ausbildung und Schaffung von sozialer Verantwortung gefördert werden (Sterner et al., 2019). Dieser Aspekt ist eng mit dem Bereich Aus- und Weiterbildung sowie der Forschung und Beratung verknüpft. Die staatlich finanzierte Aus- und Weiterbildung, die Unterstützung der Wissensgeneration im Agrarsektor und die Bereitstellung von Forschungsgeldern gehören deshalb zum agrarpolitischen Instrumentarium. In der Schweiz ist die Ausbildung kantonal geregelt. Auf Bundesebene fördert die landwirtschaftliche Beratungszentrale (AGRIDEA) den Wissens- und Erfahrungsaustausch zwischen Praxis, Beratung, Forschung, Verwaltung und Politik. Zudem verfügt der Bund mit der Agroscope über eine eigene landwirtschaftliche Forschungsanstalt. Die Forschung der Agroscope erfolgt entlang der gesamten Wertschöpfungskette der Landund Ernährungswirtschaft und soll zu einer wettbewerbsfähigen und multifunktionalen Landwirtschaft, hochwertigen Lebensmitteln für eine gesunde Ernährung sowie einer intakten Umwelt führen. Die Regierung kann aber auch versuchen, das Verhalten von Bäuerinnen und Bauern über Aufklärungs- oder Überzeugungsaktivitäten wie Werbung, Appelle oder Propaganda zu beeinflussen. Diese Formen werden unter dem Begriff persuasive Instrumente zusammengefasst (Ingold et al., 2016).

Der Staat kann auch Standards für die Präsentation von Produktinformationen setzen (Golan et al., 2001). Dies spielt eine wichtige Rolle beispielsweise im Bereich von Ursprungsbezeichnungen oder Qualitätskennzeichnungen. Durch die Festlegung von Standards und deren exakten Definition schafft der Staat die Voraussetzung, dass Informationen über das Nahrungsmittel wahrheitsgetreu dem Konsumenten präsentiert werden können. So kann verhindert werden, dass z.B. Käse mit dem Label «Alp» verkauft wird, welches eigentlich mit Milch aus dem Talgebiet hergestellt wurde. Vor dem Hintergrund der Qualitätsstrategie der Schweizer Landwirtschaft kommt diesem Instrument in der Schweizer Agrarpolitik eine wichtige Bedeutung zu. Insbesondere die Kennzeichnung mit dem Schweizer Kreuz (Swissness) ist ein wichtiges Mittel, um die inländischen Produkte im Markt zu positionieren. Im Ausland spielen vor allem 
die Ursprungsbezeichnungen eine wichtige Rolle, weil sie einen geschützten Produktionsraum schaffen. Der Staat unterhält aber grundsätzlich keine eigenen Labels. Diese entstehen aus der Kooperation zwischen den Produzenten- und Branchenorganisationen und sind in den allermeisten Fällen privatrechtlich geregelt. Die staatlichen Massnahmen sollen lediglich die Transparenz und die Grundlage für diese Kooperationen und Verhandlungslösungen schaffen.

In diesem Kontext ist in den letzten Jahren auch der Begriff Nudges aufgekommen. Damit ist gemeint, dass die Darstellung von Informationen einen Effekt auf das Kaufverhalten von Konsumentinnen und Konsumenten oder die Entscheidung des Landwirts haben kann, ohne dass dabei die eigentlichen Kosten und Nutzen des Kaufs oder der Produktionsentscheidung sich tatsächlich verändern. Solche Informationen, die versuchen, verhaltensökonomische Aspekte der Entscheidungsfindung auszunutzen, sind in der Konsumentenforschung nicht neu und können einen wichtigen Beitrag zu einem nachhaltigeren Konsum leisten (Reisch, 2021). Neuere Experimente zeigen auch, dass ein sorgfältiger Einsatz solcher Massnahmen positive Effekte haben kann. So zeigte beispielsweise ein Experiment in Frankreich, dass Landwirte, die sehr viel bewässerten, die Wassermenge reduzierten, wenn sie wöchentlich über die Bewässerungspraktiken der anderen Bäuerinnen und Bauern informiert wurden (ChabéFerret et al., 2019). Eine andere Umfrage bei französischen Bäuerinnen und Bauern zeigte ebenfalls, dass die Information über die Teilnahme von anderen Landwirten die eigene Entscheidung, an einem Agrarumweltprogramm teilzunehmen, beeinflusst (Kuhfuss et al., 2016b). Die Nutzung von verhaltensökonomischen Kenntnissen in der Umsetzung von agrarpolitischen Massnahmen ist aber noch wenig verbreitet. Im Kontext der Schweizer Landwirtschaft kommt das Programm «von Bauern für Bauern», welche die Erfahrungen von Bäuerinnen und Bauern mit einer bestimmten landwirtschaftlichen Praxis dokumentieren, der Idee von Nudges am nächsten (Schneider et al., 2012).

Der Vorteil von Informationsmassnahmen ist, dass sie normalerweise nicht zu einer Verpflichtung oder einem Zwang führen, sondern staatlich gewünschte Verhaltensweisen der Landwirtschaft lediglich anstossen. Diese Instrumente werden daher auch als Soft Tools bezeichnet (Ingold et al., 2016).

\subsubsection{Aussenhandel}

Die Regulierung des Handels ist das Kernstück vieler Agrarpolitiken. Das Ziel der Beeinflussung des Aussenhandels ist die Stärkung der inländischen Produktion. Die klassischen Instrumente sind Zölle, Zollkontingente, Exportsubventionen oder die Absatzförderung im Ausland. Als Einstieg in die Instrumente des Aussenhandels legt der folgende Abschnitt die Ursachen des Handels dar. Anschliessend werden die wohlfahrtstheoretischen Implikationen von Eingriffen in den Agrarmarkt erläutert. 


\subsubsection{Theoretische Grundlagen für den Handel mit Agrargütern}

Die Grundlage für den Handel von Agrargütern sind die komparativen Kostenvorteile (Costinot und Vogel, 2015). Komparative Kostenvorteile begründen den wohlfahrtstheoretischen Gewinn, der aus der Spezialisierung der Produktion hervorgeht. Das Lehrbuchbeispiel stammt vom Ökonomen David Ricardo (1772-1823) und behandelt den Fall von zwei Ländern (Portugal und England), die zwei Güter (Tuch und Wein) mit einem Produktionsfaktor (Arbeit) herstellen. Aus einer wohlfahrtstheoretischen Perspektive führt Handel auch dann zu einem Gewinn (mehr Produktion), wenn ein Land (in seinem Beispiel England) über Kostenvorteile in beiden Gütern verfügt. Die Logik ist, dass, wenn das Land mit den höheren Arbeitskosten sich auf dasjenige Produkt spezialisiert, für welches es relativ kleinere Nachteile besitzt, die Gesamtmenge in der Produktion erhöht werden kann. Handel entsteht folglich durch Produktivitätsunterschiede in den verschiedenen Ländern. Im Kontext der Landwirtschaft lässt sich auch empirisch festhalten, dass sich die Grundlogik der komparativen Kostenvorteile eignet, um beispielsweise die Produktion von Ackerkulturen in verschiedenen Ländern vorherzusagen (Costinot und Donaldson, 2012; Costinot et al., 2016).

Es ist wichtig, festzuhalten, dass für dieses Prinzip keine absoluten Kostenvorteile notwendig sind, wie noch von Adam Smith (1723-1790) postuliert. Absolute Kostenvorteile beschreiben den Umstand, dass eines der beiden Länder mindestens eines der beiden Güter kostengünstiger produzieren kann. Eine Kombination dieser Argumente sind im Heckscher-Ohlin-Modell zusammengefasst. Dieses Modell mit zwei Ländern, zwei Gütern und zwei Produktionsfaktoren, z.B. Arbeit und Kapital, erklärt den komparativen Vorteil nicht nur mit der unterschiedlichen Arbeitsproduktivität, sondern auch mit der unterschiedlichen Ausstattung mit Ressourcen in den jeweiligen Ländern. Nach diesem Modell werden in Ländern mit hoher Kapitalverfügbarkeit, z.B. die Schweiz, kapitalintensive Güter exportiert und arbeitsintensive Güter importiert. Die empirische Evidenz für dieses Modell ist aber unklar (Bowen et al., 1987).

Diese einfachen Handelsmodelle erklären auf einer theoretischen Basis die Vorteile des Güteraustausches zwischen zwei Ländern. Sie zeigten sich aber als unzureichend, die real stattfindenden Handelsaktivitäten auf einer empirischen Ebene zu erklären (Rodrik, 2018). Daher wurden diese grundlegenden Modelle stets weiterentwickelt bis hin zur neuen Handelstheorie von Paul Krugman (2009). Diese beinhaltet weitere Faktoren, die den Handel von Gütern erklären (Neary, 2009):

- Nachfragestruktur: In den unterschiedlichen Ländern existieren Präferenzunterschiede und die Produzenten können ihre Produkte nach Qualität, Preisen und weiteren Eigenschaften differenzieren. Eng damit verknüpft sind dabei auch die unterschiedlichen Lohnniveaus und die Kaufkraft der Bevölkerung bzw. die damit verbundenen Konsumeigenschaften (siehe dazu auch den Ab- 
schnitt zum Nahrungsmittelkonsum). Diese Unterschiede erlauben es, Spezialisierungsgewinne unabhängig von Arbeitsproduktivität oder Ressourcenausstattung zu erzielen.

- $\quad$ Economies of Scale: Steigende Skalenerträge (Economies of Scale) beschreiben eine Situation, in der eine Zunahme der Inputfaktoren zu einem überproportionalen Anstieg des Outputs führt. Das bedeutet, dass man mit einer grösseren Produktionsmenge tiefere Grenzkosten hat. Positive Skaleneffekte sind daher nicht ausschliesslich durch grosse industrialisierte Einheiten möglich, sondern können auch durch Wissensakkumulation oder Technikadaptionen erfolgen. Spezialisierungsgewinne können so auch durch den Handel zwischen Ländern mit ähnlicher Produktivität, Ressourcenausstattung und Nachfragemustern entstehen. Ein landwirtschaftliches Beispiel dafür ist der Export von dänischen Schweinen nach Japan oder auf die britischen Inseln.

- $\quad$ Produktlebenszyklus: Handelbare Produkte durchlaufen eine Art Lebenszyklus (Einführung, Wachstum, Reife, Sättigung, Degeneration). In den einzelnen Phasen können sich Produktions- und Nachfragebedingungen verändern und damit auch die Eignung für deren Produktion in unterschiedlichen Ländern, z.B. kapitalintensive Innovation bei der Einführung und Massenproduktion in der Reifephase. Die dynamische Entwicklung der Märkte über die Zeit spielt folglich eine wichtige Rolle in der Entwicklung von Handelstätigkeiten.

- Weitere Aspekte, die Handelstätigkeiten implizieren bzw. einen eingeschränkten Handel trotz theoretischen Vorteilen erklären, sind 1) die unterschiedliche Mobilität der Faktoren Kapital (tendenziell beweglich) und Arbeit (tendenziell unbeweglicher) und die damit verbundenen Anpassungskosten (siehe auch strukturelle Transformation im Kapitel 2); 2) geografische Distanz (z.B. durch Transportkosten von frischen Produkten); 3) eingeschränkte Transparenz und administrative (nicht tarifäre) Handelshemmnisse; 4) fehlende institutionelle Rahmenbedingungen (z.B. Korruption) und fehlende politische Legitimität.

- $\quad$ Strategische Handelspolitik: Aus einer empirischen Sicht lassen sich schliesslich viele Handelsmuster nur mit den politischen Massnahmen erklären, die von den unterschiedlichen Ländern eingesetzt werden. Dazu gehören, neben den im nächsten Abschnitt vorgestellten Instrumenten, auch die Währungsmanipulation, durch Abwertung oder Fixierung, oder andere Eingriffe in das gesamtwirtschaftliche Austauschverhältnis zwischen exportierten und importierten Gütern wie Terms of Trade, Dutch Disease, Infant Industries etc. (Gillis et al., 1992).

Es ist wichtig, an dieser Stelle festzuhalten, dass die Ursachen für Handel nicht als normative Grundlage für freien Handel gelten darf. Aus einer empirischen Sicht ist klar, dass Handel immer auch zu Gewinnern und Verlieren führt (Rodrik, 2018) und 
dass eine Umverteilung von Handelsgewinnen, wenn auch theoretisch nachvollziehbar, in der Realität gar nicht immer von der Gesellschaft erwünscht ist (Salvatici und Nenci, 2017). Analog zur Überlegung von Timmer (2009) ist die Frage nicht, ob wir Handel brauchen, sondern wo, zu welchen Bedingungen und zu welchem Preis.

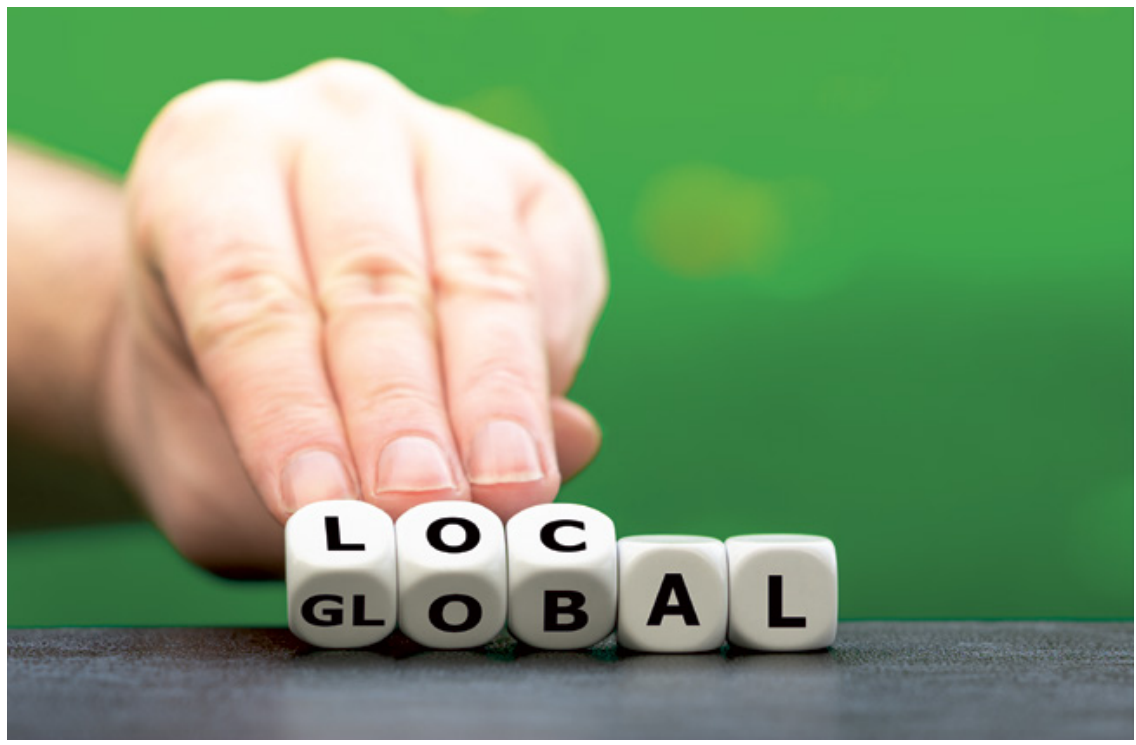

Abbildung 6. Die Kernfrage in Bezug auf den Handel mit Agrarprodukten ist, wie das Regelund Steuerungssystem unter der Berücksichtigung von Umwelt- und Sozialstandards ausgestaltet werden kann. (Bild: iStock)

Es geht also nicht darum, den Handel mit Agrarprodukten gleich ganz über Bord zu werfen (Binswanger, 2020). Das ist weder sinnvoll noch machbar, wie die Ausführungen im Kapitel 3.4 zeigten. Gleichzeitig ist das Prinzip des «Free Trade at all Cost» ebenfalls keine Option für eine nachhaltige Agrarpolitik (Rodrik, 2018). Für den Handel mit Agrarprodukten braucht es bessere Regel- und Steuerungssysteme (Governance Systems), welche die unterschiedlichen Herausforderungen des Handels aufnehmen und in die internationalen und bilateralen Verhandlungen integrieren (Koning und Pinstrup-Andersen, 2007; Walton, 2010; Martin, 2018; Friel et al., 2020; Blandford und Tangerman, 2021). Auch die Einhaltung von Umwelt- und Sozialstandards sollten darin berücksichtigt werden. Dabei spielen Plattformen wie der «Round Table on Responsible Soy» oder der «Roundtable on Sustainable Palm Oil» eine wichtige Rolle, weil sie minimale Standards für den Handel setzen können (Eyhorn et al., 2019). Ähnliche Plattformen in anderen Bereichen der globalisierten Wertschöpfungsketten könnten zu einem faireren Handel beitragen (Lambin et al., 2018). 


\subsubsection{Instrumente zur Steuerung des Aussenhandels}

\subsection{Zölle}

Die Logik des Zolls ist es, Güter zu verteuern, die aus dem Ausland importiert werden (Rieder, 1983; Henrichsmeyer und Witzke, 1994). Dadurch steigt der Preis der Güter im Inland an und die inländische Produktion kann ausgedehnt werden. Die Produzentenrente nimmt zu. Durch den höheren Preis wird aber auch weniger konsumiert und die Konsumentenrente geht zurück. Die Differenz zwischen Produzenten- und Konsumentenrente kann in drei Kategorien eingeteilt werden.

Ein Teil des Verlusts der Konsumenten wird durch Zolleinnahmen kompensiert. Durch den Zoll erhält der Staat Einnahmen, die er für Kompensationsmassnahmen nutzen könnte. Die Zolleinnahmen decken aber nicht den gesamten Verlust ab. Durch die Ausdehnung des Angebots im Inland entsteht der sogenannte Spezialisierungsverlust. Obwohl Produzenten im Ausland das Gut kostengünstiger herstellen, wird die teurere Produktion im Inland gefördert. Die Zolleinnahmen decken zudem jenen Verlust nicht ab, der durch die Reduktion des Konsums des importierten Guts entsteht. Man spricht in diesem Zusammenhang vom Handelsverlust. Die beiden Effekte zusammen, Spezialisierungsverlust und Handelsverlust, werden als Wohlfahrtsverlust (Dead Weight Losses) bezeichnet.

Wie hoch der Verlust ist, hängt wesentlich davon ab, wie hoch die Zolleinnahmen sind und ob durch den Zoll nicht nur der Preis im Inland, sondern auch der Weltmarktpreis beeinflusst wird. Der Unterschied ist, dass, wenn ein grosses Land weniger importiert, der Weltmarktpreis sinkt und das Handelsvolumen abnimmt, weil weniger nachgefragt wird. Die Zolleinnahmen, sofern sie auf einen konstanten Preis im Inland abzielen, nehmen dadurch zu. Theoretisch wäre es für ein grosses Land daher möglich, die Wohlfahrt im Inland über diese Terms-of-Trade-Effekte zu erhöhen. Das bedeutet, dass man den Weltmarktpreis so manipuliert, dass sich das Verhältnis des Preises im Inland zu den Preisen im Ausland verbessert.

Die Kosten dafür tragen aber die anderen Länder (Ausland). Sie erleiden nicht nur Effizienzverluste, Spezialisierungs- und Handelsverluste, sondern werden über die Verschlechterung der Terms of Trade doppelt bestraft. Die Weltwirtschaft als Ganzes verliert dadurch Wohlfahrt im Sinne von tieferen Produzenten- und Konsumentenrenten. Deshalb wäre es wichtig, dass multilaterale Verträge in Kraft wären und nicht nur bilaterale Abkommen. Für ein kleines Land, wie die Schweiz, sind die Effizienzverluste in jedem Fall grösser als die Zolleinnahmen und es entstehen auch im Inland Wohlfahrtsverluste (vgl. Box 6). 


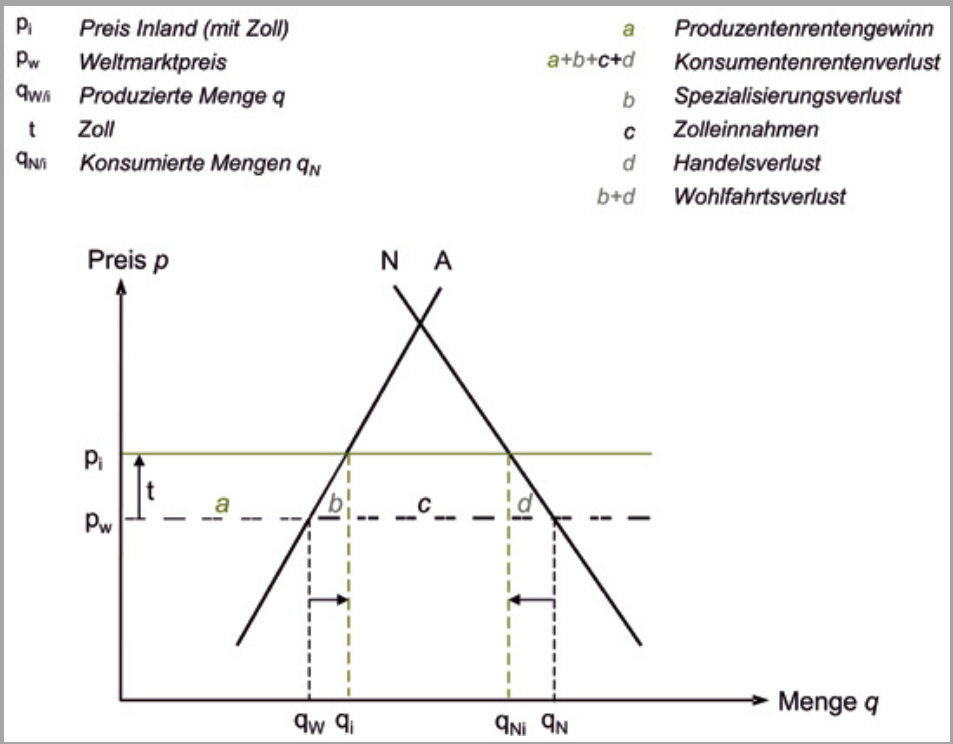

Ohne Zoll gilt im Inland der Weltmarktpreis $p_{w}$, was dazu führt, dass von den Konsumenten die Menge $q_{N}$ nachgefragt wird. Die inländischen Produzenten sind jedoch aufgrund des tiefen $p_{W}$, nur bereit, die Menge $q_{A} z u$ produzieren. Daraus resultiert eine Menge $q_{N}-q_{A}$, welche importiert wird.

Wird nun ein Zoll eingeführt, steigt der Preis im Inland von $\mathrm{p}_{\mathrm{W}}$ auf $\mathrm{p}_{\mathrm{B}}$. Der höhere Preis dient als Anreiz für die inländischen Produzenten, mehr herzustellen, d.h., sie erweitern ihre Angebotsmenge von $q_{A}$ auf $q_{A}{ }^{\prime}$. Gleichzeitig führt der höhere Preis bei den Konsumenten zu einem Rückgang der nachgefragten Menge von $q_{N}$ auf qN$^{\prime}$. Durch die Erhöhung des Angebots und den Rückgang der Nachfrage im Inland sinken die Importe (von $q_{N}-q_{A}$ auf $q_{N}^{\prime}-$ $q_{A}{ }^{\prime}$ ). Zusätzlich werden durch die Importe nun auch Zolleinnahmen (Fläche d, Importmenge * Zollsatz) generiert. Des Weiteren hat die Einführung eines Zolls einen Effekt auf die Wohlfahrt bzw. auf die Konsumenten- und die Produzentenrente. Für die Produzenten resultiert ein Rentengewinn (a), da sie mehr Güter herstellen und zu einem höheren Preis verkaufen können. Aufgrund des höheren Kaufpreises und der niedrigeren (Import-)Menge sinkt die Konsumentenrente $(\mathrm{a}+\mathrm{b}+\mathrm{c}+\mathrm{d})$. Die Zolleinnahmen und der Produzentenrentengewinn vermögen dies nicht zu kompensieren und es kommt zu einem Wohlfahrtsverlust $(b+c)$. 
Die exakte Wirkung des Zolls hängt auch von der Ausgestaltung des Instruments ab. Man unterscheidet Mengen- und Wertzölle. Letztere belasten teurere Produkte stärker. Gleitzölle oder Schwellenpreissysteme garantieren einen bestimmten Inlandpreis und passen sich dem Importpreis bzw. dem Weltmarktpreis an. Je tiefer dieser ist, desto höher der Zoll. Diese variable Abschöpfung verhindert die Weitergabe von Preisschocks und Volatilitäten. Ausserdem wird die Wirkung des Zolls auch durch die Marktsituation beeinflusst. In einer Situation ohne vollkommenen Wettbewerb, z.B. ein Monopol oder Monopson, können durch die Marktmacht zusätzliche Wohlfahrtsverluste entstehen (für die Schweiz: Jörin, 2001; Wey und Gösser, 2019).

Im internationalen Kontext ist darauf hinzuweisen, dass es auch Exportzölle gibt. Das heisst, ein Land versucht mittels eines Zolls auf die exportierten landwirtschaftlichen Güter entweder seine Staatseinnahmen zu erhöhen oder die Versorgung im Inland abzusichern (Mitra und Josling, 2009).

\subsection{Zollkontingente}

Zollkontingente sind ein zweistufiges Zollsystem (Jörin, 2001). Die Idee ist, dass für eine gewisse Menge (ein Kontingent) ein tiefer oder gar kein Zoll für den Import von Gütern angewendet wird. Ist dieses Kontingent ausgeschöpft, gilt ein zweiter hoher Zoll (vgl. Box 7). Dieses System unterscheidet sich von einem reinen Importkontingent, das vor der WTO zur Regelung der Einfuhren in die Schweiz verwendet wurde, dass ein theoretischer Marktzutritt besteht. Ausländische Anbieter können, wenn sie bereit sind, den Zoll zu bezahlen, immer in die Schweiz importieren.

Praktisch wird dieser zweite Zoll aber meistens prohibitiv hoch gesetzt, sodass der Preis im Inland nur durch die Kontingentsmenge beeinflusst wird. Der erste, tiefe Zoll wird als Kontingentszollansatz (KZA) bezeichnet. Der zweite, höhere Zoll ist der sogenannte Ausserkontingentszollansatz (AKZA). Die Höhe des Grenzschutzes hängt davon ab, in welchem Verhältnis Kontingent, KZA und AKZA zueinander stehen. Ist das Kontingent so gewählt, dass es nicht vollständig ausgeschöpft wird, reduziert sich das Zollkontingentsystem auf einen einfachen Zoll auf Höhe des KZA. Ist das Kontingent hingegen sehr klein gewählt, ist die Höhe des AKZA der entscheidende Faktor, welcher den Schutz aufrechterhält. Senkungen des AKZA führen in diesem Fall unmittelbar auch zu einem Rückgang der Produzentenpreise im Inland. Der gewünschte Schutzeffekt eines Zollkontingents wäre, dass die Kontingentsmenge ausgeschöpft wird, der AKZA aber dafür sorgt, dass keine zusätzlichen Güter importiert werden. Die maximalen Zölle und die minimalen Kontingente müssen bei der WTO hinterlegt werden und dürfen gemäss internationalem Recht nicht erhöht (AKZA) bzw. gesenkt (Kontingent) werden. 
In der Schweiz werden praktisch sämtliche landwirtschaftlichen Importe über solche Zollkontingente abgewickelt. Der Grund dafür ist, dass diese Zollkontingente in den WTO-Verhandlungen als Kompromiss hervorgingen zwischen denjenigen Ländern, die für eine Ausdehnung des Handels waren, und denjenigen, die nach wie vor Schutz für ihre Agrarprodukte forderten. Die Kontingente waren ein Zugeständnis an die Forderung einer Ausdehnung des Handelsvolumens. In den laufenden Verhandlungen gehören die Ausdehnung der Kontingente und die Senkung der AKZA zu den Kernelementen einer Weiterentwicklung des Agreement on Agriculture.

Die wohlfahrtsökonomischen Auswirkungen des Zollkontingents sind ähnlich wie beim einstufigen Zoll: Einheimische Produzenten gewinnen, einheimische Konsumenten verlieren, der Staat erhält Zolleinnahmen. Allerdings besteht ein wesentlicher Unterschied: Auf der Kontingentsmenge sind die Zolleinnahmen aufgrund des tiefen KZA klein. Der Konsumentenrentenverlust bleibt jedoch analog zur Situation mit Zoll hoch. Diese Gewinne, d.h. der Unterschied zwischen dem Preis für die Konsumenten und dem Importpreis, werden als Importrente bezeichnet. Diese Rente fällt nicht mehr dem Staat zu, sondern den Importeuren. Für die Wirkungsweise von Zollkontingenten ist nun entscheidend, wie diese Gewinne verteilt werden. Ursprünglich wurde ein System angewandt, bei dem die inländischen Verarbeiter proportional zur Menge aus dem Inland ein Anrecht auf ein Importkontingent erhielten. Dieses System wird als Inlandleistung bezeichnet und wird im Bereich von Früchten und Gemüsen weiterhin angewendet.

Ein Problem ist, dass dadurch die Marktmacht der ohnehin schon dominierenden Unternehmen, z.B. Migros und Coop, zementiert wurde. Die marktmächtigen Unternehmen konnten aufgrund ihrer hohen Inlandleistung auch die Importkontingente verwalten, wodurch der Markt intransparenter wurde. Aus diesem Grund wurde für viele Produkte ein Versteigerungsverfahren eingeführt. Üblicherweise können Händler und Verarbeiter einen Preis für einen bestimmten Teil des Kontingents bieten. Die Teilnehmenden haben die Möglichkeit, maximal fünf verschiedene Gebote einzureichen. Anschliessend wird die Kontingentsmenge ausgehend vom höchsten gebotenen Preis in abnehmender Reihenfolge verteilt. Diese Art der Versteigerung wird in der Schweiz für die Kategorien Fleisch, Wurstwaren, Schnittblumen, Kartoffeln, Schafe, Rinder und Milchprodukte angewendet. Je nach Produkt wird die Versteigerung aber nur für einen Anteil des Kontingents durchgeführt. Die restlichen Anteile werden nach wie vor über die Inlandleistung zugeteilt, z.B. bei Rindfleisch. Für saisonale Produkte wie Früchte oder Gemüse werden die Zollkontingente auch auf sogenannte bewirtschaftete Perioden beschränkt und erfolgen aufgrund der Inlandleistung. Ausserhalb der Saison (nicht bewirtschaftete Periode) gelten keine Einfuhrrestriktionen. Neben der Versteigerung kann die Zuteilung der Importkontingente 


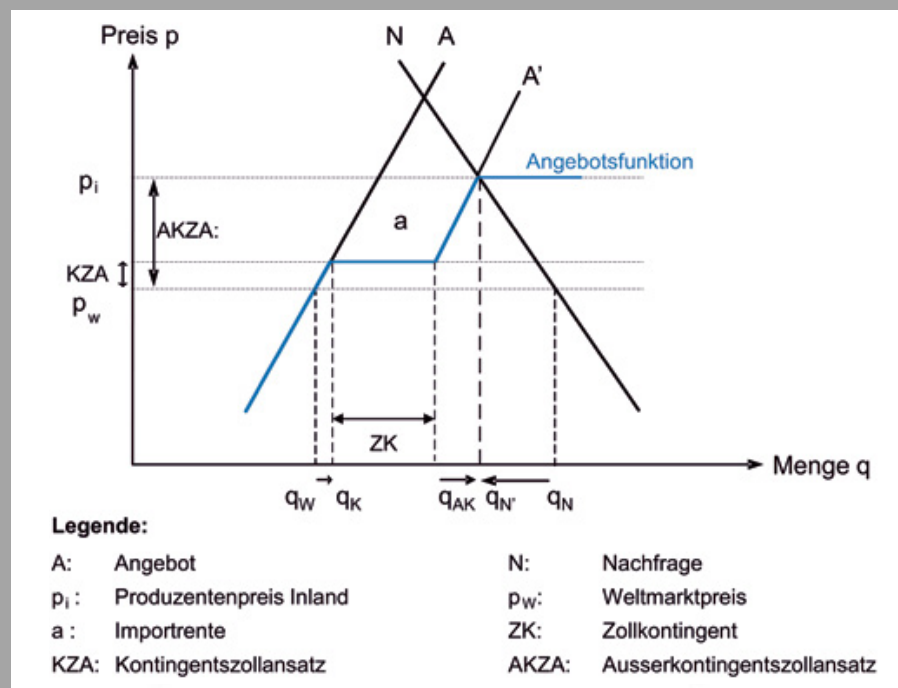

Ohne Grenzschutz würde der Weltmarktpreis $p_{w}$ gelten und im Inland würden die Mengen $q_{w}$ produziert und $q_{N}$ nachgefragt. Die Menge $q_{N}-q_{A}$ wird importiert. Ein Zollkontingent hat zum Ziel, den Preis im Inland und damit die Produzentenrente zu erhöhen. Das Zollkontingent besteht aus zwei Zollansätzen. Für eine vorbestimmte Menge, das sogenannte Zollkontingent ZK, gilt ein tiefer Zollkontingentsansatz (ZKA). Dieser erhöht den Preis im Inland, sodass die Produzenten ihre Menge von $q_{w}$ auf $q_{k}$ erhöhen können. An dieser Stelle wird die Angebotsfunktion ein erstes Mal «geknickt», da die Zollkontingentsmenge die Angebotsfunktion der inländischen Produzenten von A nach $A^{\prime}$ verschiebt. Ist das Zollkontingent ausgeschöpft, wird für die zusätzlichen Importe ein meist prohibitiv hoher Ausserzollkontingentsansatz (AKZA) angesetzt. Dadurch wird der Preis auf das Niveau $p_{i}$ angehoben. Die Produzenten können dadurch ihre Menge weiter auf $q_{A K}$ ausdehnen. Gleichzeitig geht die Nachfrage um die Menge $q_{N}-q_{N^{\prime}}$ zurück. Die Angebotsfunktion wird an dieser Stelle ein zweites Mal «geknickt», weil die Importe in jedem Fall wieder günstiger sind als die inländische Produktion. In diesem Beispiel wird nur das Zollkontingent eingeführt und es finden keine Importe zum AKZA statt. Mit einem Zollkontingent ist dadurch die im Inland produzierte Menge grösser und die konsumierte Menge kleiner als unter Weltmarktpreisen. Zusammen mit dem höheren Preis nimmt dadurch die Produzentenrente zu und die Konsumentenrente ab. Ausserdem entsteht eine Importrente a, da das ZK zu einem tieferen Preis eingeführt, aber zum höheren Preis $p_{\mathrm{i}}$ verkauft werden kann. 
auch über ein sogenanntes Windhundverfahren (First Come, First Serve) erfolgen. Die Zuteilung von Globalkontingenten für Wein, Eier, Pferde und Brotgetreide erfolgt gemäss dem Eingang der Gesuche zu Beginn des Jahres.

Zollkontingente sind ein effektives Instrument, um die Produzentenrente im Inland zu erhöhen (Hillen, 2019). Je nach Marktstruktur können aber zusätzliche Effizienzverluste entstehen, weil die Transparenz in Bezug auf die Importrenten auf der Stufe Gross- und Detailhandel verloren geht. Dadurch werden die Margen in den nachgelagerten Unternehmen erhöht (Loi et al., 2016). Im Kontext des Schweizer Zollsystems ist zu erwähnen, dass für die am wenigsten fortgeschrittenen Entwicklungsländer (Least Developed Countries, LDC) Zollbegünstigungen bestehen, um den Marktzugang zu erleichtern. So beträgt beispielsweise der Zoll für Sonnenblumenöl aus Ländern wie Mosambik, Tansania oder Uganda null Franken.

\subsection{Exportsubventionen}

Exportsubventionen bewirken, dass Produkte aus dem Inland an der Grenze verbilligt werden, damit im Ausland mehr davon abgesetzt werden kann (Henrichsmeyer und Witzke, 1994). Der Staat kauft die inländische Produktion auf und setzt sie auf dem Weltmarkt ab. Die Preisdifferenz wird durch den Steuerzahler ausgeglichen. Der Effekt ist, dass üblicherweise der Preis im Ausland oder bei grossen Agrarexporteuren der Weltmarktpreis sinkt. Von einer Exportsubvention profitieren daher die Produzenten im Inland und die Konsumenten im Ausland. Hingegen werden die Produzenten im Ausland benachteiligt und im Inland fallen unter Umständen hohe Kosten an, um die Güter zu verbilligen. Das bedeutet, dass Exportsubventionen zu ähnlichen Effizienzverlusten führen wie Zölle, sie aber in jedem Fall die Terms of Trade verschlechtern und dadurch zu hohen Wohlfahrtsverlusten führen.

Dieser offensichtliche Missstand ist schon lange bekannt und eine der ersten Massnahmen der WTO war es, diese Exportsubventionen zu verbieten. Erst in der NairobiKonferenz der WTO 2015 wurde ein Ablaufdatum für die Exportsubventionen festgelegt. Die Schweiz beispielsweise verbilligte die Rohstoffe Butter, Milchpulver und Mehl für Exportprodukte bis 2019. Mit dem System der Zulagen (siehe Kapitel Agrargesetzgebung) wird diese Verbilligung praktisch weitergeführt. Der Grund für das Festhalten an der Verbilligung ist, dass das Absetzen von Mehrmengen aufgrund der grösseren Preiselastizität im Ausland attraktiv ist. Relativ zum Inland können mit einer Preissenkung im Ausland mehr Produkte abgesetzt werden. Wichtig ist zu wissen, dass die Exportsubventionen zu einer Art Dumping führen und daher mit Sicherheit die wohlfahrtsökonomisch schlechteste Variante des Aussenhandels sind.

\subsection{Weitere Instrumente im Bereich des Aussenhandels}

Zölle und Zollkontingente sind die zentralen Instrumente zur Regelung des Aussenhandels in der Landwirtschaft. Die Beeinflussung des Aussenhandels mit dem Ziel, die inländische Produzentenrente zu erhöhen, kann ebenso über Kombinationen oder leicht 
abgeänderte Instrumente erfolgen. So werden in der Literatur auch Abschöpfungssysteme (Verteuerung der Importe), Deficiency Payment Systems (Verbilligung des inländischen Angebots) oder Importmonopole beschrieben. Die Umsetzung dieser Systeme ist zwar unterschiedlich, in ihrer grundsätzlichen Wirkung unterscheiden sie sich jedoch nicht von Zöllen oder Exportsubventionen. Auch Nahrungsmittelhilfe kann grundsätzlich wie eine Exportsubvention wirken, wenn sie nicht zeitlich begrenzt wird. Schliesslich kennen grosse Agrarexporteure auch Exportsteuern. Mit Steuern auf Agrarprodukten kann der Staat, sofern genügend Lebensmittel produziert werden, einfach Einnahmen generieren (Beispiel: Argentinien besteuerte bis 2015 Soja und Rindfleischexporte). Schliesslich existieren, trotz WTO, nach wie vor nicht tarifäre Handelshemmnisse. Dazu gehören beispielsweise die Abfertigung am Zoll mit unterschiedlichen Kennzeichen oder unterschiedlichen Deklarationsvorschriften.

\subsubsection{Steuerung von Angebot und Nachfrage im Inland}

Neben der Beeinflussung des Aussenhandels kommen auch den Instrumenten zur Steuerung von Angebot und Nachfrage im Inland eine hohe Bedeutung in der Agrarpolitik zu. Klassische Beispiele dafür sind die Kontingentierung, Marktspaltungen, Absatzförderung oder die Unterstützung von Strukturen oder Wertschöpfungsprojekten.

\subsubsection{Kontingentierung}

Der Grenzschutz ist ein undifferenziertes Instrument, d.h. nicht tailored. Von höheren Produzentenpreisen profitieren alle Betriebe, die das entsprechende Produkt herstellen. Da der einzelne Landwirt den Preis mit seiner produzierten Menge nicht beeinflussen kann, hat er keinen Anreiz, seine maximale Produktion nicht auszuschöpfen. Über den gesamten Sektor gerechnet bedeutet dies, dass mit höheren Preisen automatisch auch eine Produktionsausdehnung stattfindet. Dieser Effekt wird grösser, je sicherer der Absatz für die Landwirte ist. In den 1970er-Jahren garantierte der Bund in der Schweiz die Abnahme der landwirtschaftlichen Produkte zu einem jährlich festgelegten Preis. Die Folge davon war, dass die Landwirte die Produktion weit über die im Inland nachgefragte Menge erhöhten, insbesondere im Milchsektor. Als Folge dieser Preis-Absatz-Garantie musste daher eine Kontingentierung eingeführt werden (Rieder und Anwander Phan-huy, 1994).

Die Idee der Kontingentierung im Inland ist es, einen festgelegten Zielpreis zu erreichen, indem man die Angebotsmenge beschränkt (vgl. Box 8, Diagramm links). Theoretisch knickt daher die Angebotsfunktion bei der kontingentierten Menge und verläuft senkrecht nach oben. Eine Kontingentierung kann für die Begrenzung von Produktionsfaktoren oder Produktionsmengen eingesetzt werden. Ein Beispiel für die Kontingentierung von Faktoren ist die Beschränkung der Anbaufläche von spezifischen Kulturen wie z.B. Raps oder Zuckerrüben über Verträge zwischen den Landwirten und den Abnehmern. 


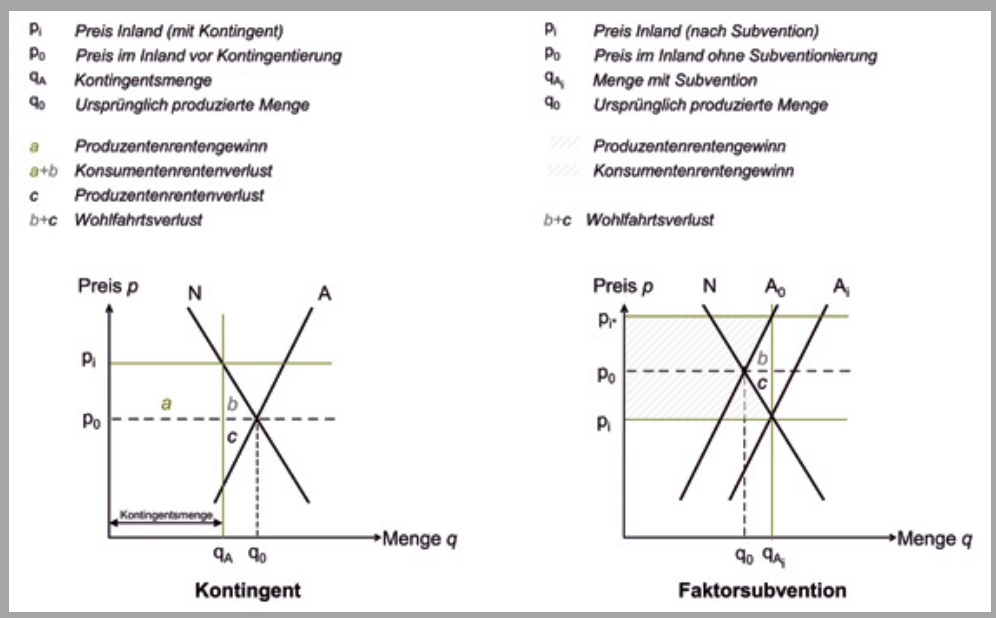

In den beiden Diagrammen ist die Ausgangssituation, d.h. das Marktgleichgewicht jeweils mit den Preisen $p_{0}$ und Mengen $q_{0}$ gekennzeichnet. Wird die Produktionsmenge kontingentiert (linkes Diagramm), dann wird eine Menge $q_{A}$ festgelegt, welche die Angebotsfunktion senkrecht werden lässt. Dadurch steigt der Preis im Inland auf die $\mathrm{p}_{\mathrm{i}}$. Wichtig ist zu bemerken, dass dieser Anstieg nur funktioniert, wenn die Mengenbegrenzung nicht durch Importe substituiert werden kann. Die Produzentenrente steigt durch die Kontingentierung um die Fläche a. Allerdings nimmt die Produzentenrente auch im Umfang c ab, weil eigentlich konkurrenzfähige Produzenten ihre Mengenkapazitäten nicht ausschöpfen können. Gleichzeitig nimmt die Konsumentenrente um die Fläche $a+b a b$. Der Netto-Wohlfahrtsverlust beläuft sich damit auf die Flächen $b+c$.

Mit einer Faktorsubvention (rechtes Diagramm) wird die Angebotsfunktion nach rechts verschoben von $A_{0}$ nach $A_{i}$. Damit dies geschieht, muss der Bund die Differenz zwischen der neu produzierten Menge und den Kosten ausgleichen, welchen der Landwirtschaft entstehen. Da sich die Kostenstruktur der Anbieter nicht verändert hat, muss dafür eine Subvention so hoch sein, dass die Produzenten den Preis $p_{i *}$ erhalten. Nur dann dehnt sich das Angebot auf $\mathrm{q}_{\mathrm{Ai}}$ aus. Mit der Subvention gewinnen die Produzenten, über den höheren Preis $\mathrm{p}_{\mathrm{i}}$ beim Angebot, als auch die Konsumenten, welche vom tieferen Preis $p_{i}$ auf der Nachfrageseite profitieren. 
Die Renten sind jeweils schraffiert dargestellt. Diese Rentengewinne sind aber nicht so hoch wie die Subvention, welche über die gesamte produzierte Menge ausbezahlt wird $\left(q_{A i} \times\left(p_{i} *-p_{i}\right)\right)$. Es entsteht ein Wohlfahrtsverlust der Fläche b und c. Im Kontext der Schweizer Agrarpolitik ist z.B. die Verkäsungszulage ein Beispiel für eine Faktorsubvention. Diesbezüglich wird hier unterstellt, dass die Subvention direkt zu einer Reduktion der Konsumentenpreise führt und nicht in der Wertschöpfungskette absorbiert wird (siehe dazu auch die empirische Analyse von Finger et al., 2017).

Das klassische Beispiel für die Produktion in der Schweiz war die Kontingentierung der Verkehrsmilch (1977-2009). Jeder Landwirtschaftsbetrieb erhielt ein Kontingent zugewiesen, für welches er einen höheren Preis erhielt. Milch, die darüber hinaus produziert wurde, konnte nur zu einem sehr viel tieferen Preis verkauft werden. Kontingentierung funktioniert allerdings nur, wenn ein abgeschlossener Markt bzw. ein effektiver Grenzschutz bestehen. Ansonsten würde die Angebotsfunktion nicht senkrecht geknickt, sondern waagrecht auf Stufe des Weltmarktpreises verlaufen.

Die wohlfahrtstheoretische Betrachtung zeigt, dass mit einer Kontingentierung der Verlust der Konsumentenrente in jedem Fall grösser ist als der Gewinn für die Produzenten (Kontingentsrente). Es entstehen Wohlfahrtsverluste. Oft sind mit der Vergabe und der Kontrolle von Kontingentssystemen auch hohe administrative Kosten verbunden. Zudem muss die Menge der Nachfrageentwicklung angepasst werden. Die Preissignale des Marktes müssen über einen administrativen Prozess an die Betriebe weitergegeben werden. In der Darstellung der Kontingentsrente mit einer senkrechten Angebotsfunktion geht man zudem implizit von einer effizienten Verteilung der Kontingentsrechte aus, d.h., die am ineffizientesten produzierten Einheiten werden nicht mehr produziert. In der Realität entsteht aber ein zusätzlicher Regulierungsverlust, weil auch ineffizientere Betriebe ein Kontingent erhalten. Dieser Effekt wird durch die Dynamik des Strukturwandels verstärkt. Die individuell festgelegten Kontingente führen dazu, dass auch Betriebe mit hohen Kosten die Produktion weiterführen. Wachsende Betriebe mit einer besseren Kostenstruktur können ihre Kapazität nicht auslasten, weil die Mengen ausserhalb des Kontingents oft mit prohibitiv tiefen Preisen verbunden sind. Würde der erste Betrieb aufhören zu produzieren und der grössere Betrieb die Produktion übernehmen, so könnte die gleiche Menge Milch zu tieferen Kosten hergestellt werden. Die Schweiz erlaubte vor der Aufhebung der Kontingentierung, dass Kontingente für Verkehrsmilch gehandelt werden durften, um diesen strukturellen Effekt einzudämmen. Handelbare Kontingentsrechte reduzieren zumindest teilweise den Regulierungsverlust einer ineffizienten Zuteilung der Rechte. Da die Differenz in den Produktionskosten oft hoch war, entstand ein 
reger Handel von Kontingentsmengen. Die hohe Nachfrage nach Kontingenten führte aber wiederum zu hohen Preisen, wodurch die Kosten für die kostengünstigeren Betriebe wieder zunahmen. Dieser Rückkopplungseffekt führte schliesslich zur Aufgabe der Kontingentierung in der Schweiz.

Die aktuelle Regelung im Schweizer Milchmarkt sieht eine Preisdifferenzierung für unterschiedliche Marktsegmente vor (Reviron und Python, 2018). Für die im Inland abgesetzte Milch wird ein höherer A-Milchpreis ausbezahlt. Für Mengen, die nicht im Inland, aber in angrenzenden Märkten abgesetzt werden können, wird ein tieferer B-Milchpreis bezahlt. Milch, die darüber hinaus auf den Weltmarkt geht, erzielt einen C-Milchpreis. Auch diese Marktspaltung und Preisdifferenzierung folgt im Grunde der Logik einer Kontingentierung. Der Landwirt erhält einen Anreiz, keine Milch zu produzieren, die er nicht zu einem A-Milchpreis absetzen kann. Der Unterschied zur Kontingentierung ist, dass es sich im aktuellen System um eine privatrechtliche Regelung mit Standardverträgen handelt, die Mengen aber nicht allgemeinverbindlich gesteuert werden (Python et al., 2018). Die Ausgestaltung der Mengen und Preise wird also den privaten Marktteilnehmern überlassen und nicht mehr durch den Staat orchestriert.

\subsubsection{Subventionierung von Produktionsfaktoren}

Um die Produktion wettbewerbsfähiger zu machen und das Angebot von Agrarprodukten im Inland zu stärken, kann die Agrarpolitik auch Produktionsfaktoren subventionieren (vgl. Box 8, rechtes Diagramm). Diese Subventionierung kann auf verschiedenen Stufen der Wertschöpfungskette stattfinden. Klassische Faktorsubventionen auf Stufe der Produzentinnen und Produzenten sind beispielsweise die staatliche Beteiligung an Krediten über sogenannte Strukturverbesserungsmassnahmen (Kapitalverbilligung) oder eine Verbilligung von Inputfaktoren wie Treibstoffen, Dünger, Pflanzenschutzmitteln oder Versicherungen. Auf der Stufe Verarbeitung ist beispielsweise die Milch wiederum ein Produktionsfaktor. Die Verbilligung der Milch zur Käseherstellung (Verkäsungszulage) ist ein Beispiel für die Faktorsubventionierung auf Verarbeitungsstufe.

Die Logik hinter einer Subvention von Produktionsfaktoren ist, dass sich die Angebotsfunktion ausgehend von einem Marktgleichgewicht nach rechts verschiebt. Damit sinkt der Preis für den Abnehmer und er übernimmt eine grössere Menge. Die Subvention an den Produzenten kompensiert diesen Verlust, so als ob er einen höheren Preis erzielt hätte. Die Faktorsubventionierung führt zu einem Wohlfahrtsverlust, weil die Kosten der Subvention den Gewinn der Produzenten und Konsumenten überkompensieren. Der Grund ist, dass Faktoren eingesetzt werden, die in einer alternativen Aktivität produktiver wären. Auf Stufe der Verarbeitung kann die Subvention auch als Verschiebung der Nachfragefunktion der Abnehmer betrachtet werden (Finger et al., 2017). Der Wohlfahrtseffekt bleibt jedoch der gleiche. Im Falle der 
Verkäsungszulage kommt hinzu, dass der Faktor (in diesem Fall die Milch) auf verschiedene Märkte verteilt werden kann (weisse und gelbe Linie). Entsprechend kompliziert wird die Evaluation dieser Massnahme. Die aktuellste Untersuchung (Finger et al., 2017) zeigt, dass das Instrument die Produzentenpreise effektiv stützt, die Wirkung aber stark sorten- und damit marktabhängig ist.

\subsubsection{Absatzförderung}

Das klassische Instrument zur Förderung der Nachfrage im Inland ist die sogenannte Absatzförderung. Diese besteht aus der finanziellen Förderung von Kommunikationsmassnahmen der Produzenten- und Branchenorganisationen zur Steigerung des Absatzes landwirtschaftlicher Produkte und Dienstleistungen (Rieder et al., 2015). Das Ziel der Absatzförderung ist es, dass mehr inländische Produkte nachgefragt werden und sich dadurch die Nachfragefunktion nach rechts verschiebt. Dadurch nimmt die Zahlungsbereitschaft für die gleiche Absatzmenge zu. Analog zur Subventionierung der Faktoren besteht aus wohlfahrtsökonomischer Sicht das Problem, dass die staatlichen Ausgaben höher sind als der Gewinn der Produzenten und Konsumenten. Allerdings ist die Evaluation dieses Verlustes schwer zu quantifizieren, weil eine Verschiebung der Nachfragefunktion von ganz vielen anderen Faktoren, z.B. Bevölkerungsentwicklung, Trends im Nahrungsmittelkonsum etc., ebenfalls beeinflusst wird. Wie gross der Effekt der staatlichen Unterstützung für die Werbung von landwirtschaftlichen Produkten tatsächlich ist, lässt sich daher nicht eindeutig feststellen.

\subsubsection{Weitere Instrumente im Bereich Angebot und Nachfrage im Inland}

Weitere Instrumente sind i) die finanzielle Förderung von Wertschöpfungsprojekten, die den Absatz von lokalen oder regionalen Produkten unterstützen. Aus einer wohlfahrtsökonomischen Perspektive stellt sich auch hier die Frage, wie hoch die Opportunitätskosten sind, d.h. die Verwendung der Subventionen in alternativen Aktivitäten. ii) Die Subventionierung des Konsums über Nahrungsmittelgutscheine, üblicherweise für die Bevölkerung aus tiefen Einkommensschichten wie in den USA, führt zu einem Anstieg der Nachfrage. Eigentlich als Sozialhilfemassnahme gedacht, führt sie indirekt auch zu einer Erhöhung der Produzentenrente.

\subsection{5 Ökonomische Instrumente der Agrarumweltpolitik}

Auflagen und Command-and-Control-Instrumente sind wichtige Bestandteile der Umweltpolitik in der Landwirtschaft. Man kann umweltschädliche Praktiken schlicht und einfach verbieten. In den letzten Jahren hat aber die Bedeutung von marktorientierten Instrumenten zugenommen. Im Folgenden werden drei unterschiedlich ökonomische Anreizsysteme vorgestellt: Steuern, Subventionen und Versteigerungen.

\subsubsection{Steuern und Lenkungsabgaben}

Eine grundlegende agrarökonomische Regel ist, dass der Faktorpreis, z.B. der Preis für Dünger, im Optimum dem Wertgrenzprodukt, z.B. dem Erlös aus einer zusätzlichen Einheit Weizen, entsprechen muss. Umgekehrt bedeutet dies, dass mit der 
Verteuerung des Inputfaktors die Inputintensität im Optimum abnimmt. Diese Gesetzmässigkeit macht sich die Steuer zu nutzen, um die Umweltbelastung in den Produktionsentscheid der Landwirte zu integrieren.

Aus einer wohlfahrtsökomischen Sicht stammt die Idee, dass eine Steuer die Angebotsfunktion der Landwirtschaft nach oben verschiebt oder dreht. Das bedeutet, dass bei konstantem Produzentenpreis das Angebot zurückgeht und dadurch die damit verbundene Umweltbelastung ebenfalls abnimmt (Lusk, 2013). Diese Idee der Besteuerung wird als Pigou-Steuer bezeichnet (nach dem Ökonomen Arthur Cecil Pigou 1877-1959). Die Höhe der Steuer wird in diesem Konzept über die sogenannte soziale Angebotsfunktion bestimmt. Diese Funktion beschreibt nicht nur den Verlauf der Produktions-, sondern auch diejenigen der Schadenskosten. Das Problem ist, dass diese soziale Angebotsfunktion nur sehr schwer herzuleiten ist, weil sie einer gesellschaftlichen Bewertung der Schadenskosten bedarf, z.B. durch eine Monetarisierung. Dies ist insbesondere deshalb schwierig, weil die Steuer zu einer Reduktion der Produzenten- und Konsumentenrenten führt (siehe Box 9).

Im Unterschied zu einer reinen Steuer wird bei der Lenkungsabgabe die Einnahme aus der Besteuerung von negativen Externalitäten in den landwirtschaftlichen Sektor zurückgeleitet, um beispielsweise emissionsarme Produktionstechnologien zu fördern. Im Kontext der Agrarpolitik werden in erster Linie Lenkungsabgaben im Kontext von Pflanzenschutzmitteln und Stickstoff diskutiert (Kanter et al., 2020a; Möhring et al., 2020). Die Wirkung dieser Massnahme hängt wesentlich von der konkreten Umsetzung ab. Im Bereich der Pflanzenschutzmittel könnten die Einnahmen aus der Lenkungsabgabe auf besonders umweltschädliche Spritzmittel beispielsweise dafür genutzt werden, moderne Spritzapplikationen oder den Einsatz von resistenten Sorten zu subventionieren. Im Kontext von Stickstoff könnte beispielsweise eine Steuer auf Emissionen von Futtermitteln oder Handelsdüngern für die Unterstützung von Agrarumweltprogrammen im Bereich Biodiversität eingesetzt werden (Schläpfer, 2016). Steuern und Lenkungsabgaben sind allerdings in der Landwirtschaft unbeliebt, weil sie die Produktionskosten der Landwirte direkt erhöhen und unterschiedliche Produktionszweige unterschiedlich stark beeinflussen. Aus einer wohlfahrtsökonomischen Sicht führt eine Steuer aber zu einer direkten Internalisierung der negativen Umwelteffekte in das Preissystem der Landwirte.

\subsubsection{Abgeltung für Umweltleistungen}

Wenn die Produktion von Nahrungsmitteln mit positiven Externalitäten verbunden ist, dann kann eine Abgeltung oder Subvention zu einer Erhöhung der Wohlfahrt führen (siehe Box 9). Die Abgeltung für Umweltleistungen erfolgt in der Schweizer Agrarpolitik grundsätzlich über eine Zahlung vom Staat zum Landwirt. Abgeltungen für Umweltleistungen können aber auch breiter definiert werden und auch private Zahlungen, z.B. von Umweltorganisationen, an Landwirte mit einschliessen. Im wissen- 
schaftlichen Diskurs hat sich in den letzten Jahren der Begriff der «Payments for Ecosystem Services» etabliert (Engel et al., 2008; Jack et al., 2008; Engel, 2016). Der wissenschaftliche Diskurs ist zwar nicht immer eindeutig und es existieren verschiedene Definitionen, was genau nun «Payments for Ecosystem Services» seien. An dieser Stelle ist aber wichtig, dass die Kriterien zur Beurteilung dieser Zahlungen auch sehr gut auf die Schweizer Agrarumweltprogramme passen. Neben den allgemein gültigen Ansprüchen an das Targeting und Tailoring, welche auch für Umweltzahlungen gelten, gibt es weitere Kernelemente, die in der Beurteilung dieser Zahlungen wichtig sind (Engel, 2016):

1) Konditionalität und Additivität: Zentral für die Implementierung einer solchen Zahlung ist, dass diese tatsächlich von einer Umweltleistung abhängig ist. Das mag trivial klingen, ist aber nicht immer so einfach umzusetzen, z.B. weil die Faktoren, die zur Umweltleistung führen, nicht eindeutig bestimmbar sind (siehe dazu die Dimension Input-Outcome-Beziehung). Ausserdem soll die Einführung einer Zahlung dazu führen, dass eine Verbesserung der Umweltsituation entsteht (Additivität). Hier ist zu bemerken, dass die Additivität einer Massnahme massgeblich von der Ausgangssituation abhängt, z.B. ob man mehr von einer Umweltleistung möchte oder nur den Rückgang stoppen will (siehe dazu die Ausführungen im Kapitel Wirkungsmodell).

2) Leakage und Dauerhaftigkeit: Eine Abgeltung sollte dazu führen, dass negative Externalitäten tatsächlich reduziert und nicht einfach über den Raum oder die Zeit verschoben werden. Wenn beispielsweise mit der Reduktion von negativen Effekten eine Zunahme von Importen von landwirtschaftlichen Gütern verbunden ist, kann dies zu einer räumlichen Verschiebung der negativen Externalitäten führen. In diesem Fall werden negative Effekte aus der landwirtschaftlichen Produktion im Inland auf Kosten der Umweltqualität in anderen Ländern verringert. Es ist auch möglich, dass negative Effekte sich erst in der Zukunft manifestieren wie beispielsweise beim Klimawandel. Der Umstand, dass negative Externalitäten durch eine Politikmassnahme über den Raum oder die Zeit verschoben werden können, wird als Leakage bezeichnet (Meyfroidt et al., 2020). Eine weitere Form des Leakage kann darin bestehen, dass Betriebstypen unterschiedlich auf Anreizzahlungen reagieren. Im Kontext der Schweizer Agrarpolitik wird über eine Verschärfung des ökologischen Leistungsnachweises nachgedacht, so beispielsweise im Rahmen der Trinkwasserinitiative, die 2021 vom Volk abgelehnt wurde. Auch da bestand die Gefahr des Leakage, indem landwirtschaftliche Betriebe auf die Erfüllung des ökologischen Leistungsnachweises verzichtet und dadurch den positiven Umwelteffekt der Initiative geschmälert hätten (Schmidt et al., 2019). Wenn immer möglich, sollten solche Effekte verhindert oder minimiert werden. Ausserdem sollen Massnahmen dauerhaft sein. Das bedeutet, dass bei der Ausgestaltung der Massnahme, natürlich in Abhängigkeit vom Umweltziel, die Dauer der Teilnahme, z.B. über eine Mindestdauer, festgelegt werden sollte. 
In der Schweiz werden Abgeltungen für Umweltleistungen als ökologische Direktzahlungen bezeichnet. Sofern der Landwirt den ökologischen Leistungsnachweis erfüllt, der die minimalen Umweltschutzaspekte der Landwirtschaft aus Sicht des Gewässer-, Boden- und Tierschutzes abdeckt, werden zusätzliche, freiwillige Leistungen über Zahlungen abgegolten. Diese Leistungen können einerseits eine Reduktion von negativen Externalitäten beinhalten, z.B. den Verzicht auf Kraftfutter in der Milchund Fleischproduktion, oder eine Abgeltung für positive Leistungen darstellen, z.B. für Biodiversitätsförderflächen. Analog zu einer Steuer ermöglicht eine Subvention die Internalisierung einer positiven Externalität, z.B. Landschaftspflege, oder die Reduktion einer negativen Externalität, z.B. Reduktion Stickstoffüberschüsse, in das Entscheidungssystem des Landwirtes. Im letzteren Fall ist die Zuweisung der Eigentumsrechte entscheidend.

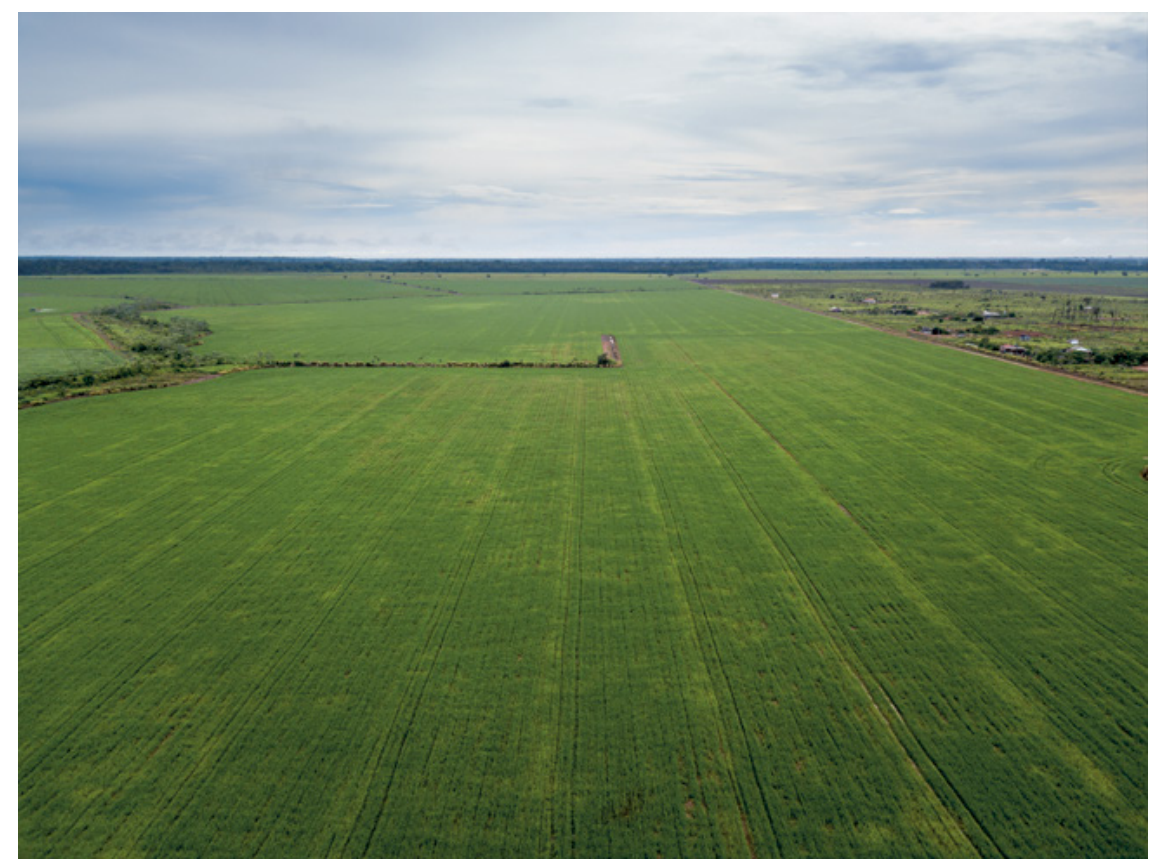

Abbildung 7. Politikmassnahmen können ungewollt dazu führen, dass negative Externalitäten über den Raum verschoben werden (Leakage). Wird die inländische Produktion reduziert und durch (unregulierte) Importe ersetzt, kann dies negative Externalitäten verlagern wie beispielsweise durch eine Steigerung des Sojaanbaus im Amazonasgebiet. (Bild: Paralaxis/iStock) 
Einführung in die Schweizer Agrarpolitik

Box 9 Wohlfahrtsgewinne durch die Internalisierung von positiven und negativen Externalitäten

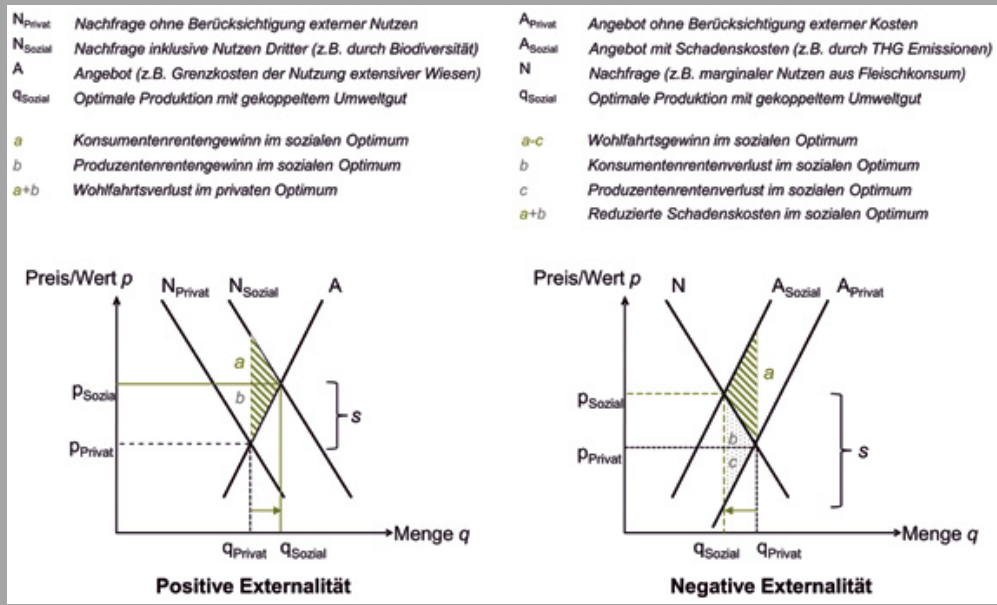

In den beiden Diagrammen ist die Ausgangssituation jeweils als privates Optimum dargestellt, d.h., die an die Produktion gekoppelten Nutzen oder Kosten sind im Marktgleichgewicht nicht im Preis inbegriffen.

Im Falle der positiven Externalität ist der marginale Nutzen beim Konsum eines Gutes höher, weil nicht nur privater Nutzen anfällt, sondern auch Dritte davon profitieren, z.B durch die Erhaltung von Biodiversität. Die eigentliche Nachfragefunktion verschiebt sich daher nach rechts (linkes Diagramm).

Geht man von der Nachfragefunktion $\mathrm{N}_{\text {sozial }}$ aus, dann verschiebt sich das Optimum (d.h. dort, wo die Grenzkosten und der marginale Nutzen gleich sind) nach rechts. Im Gleichgewicht wird daher mehr des Gutes q angeboten ( ( $\left._{\text {sozial }}\right)$. Dadurch nehmen sowohl die Konsumentenrente $a$ als auch die Produzentenrente $b$ zu. Wird im privaten Optimum produziert, gehen diese Renten verloren und es entsteht ein Wohlfahrtsverlust durch die fehlende Internalisierung von positiven Externalitäten. Eine Internalisierung lässt sich beispielsweise durch eine Subvention s (z.B. Direktzahlung) in der Höhe der Differenz zwischen psozial - P Privat bewerkstelligen. Die Kosten der Subvention trägt der Steuerzahler. 
Im Falle von negativen Externalitäten (rechtes Diagramm) entstehen mit jeder produzierten Einheit zusätzliche Kosten (Schadenskosten wie z.B.

THG Emissionen). Das bedeutet, dass die eigentlichen Kosten höher sind als die privaten Produktionskosten. Die Angebotsfunktion (d.h. die Aggregation der Grenzkosten) verschiebt sich daher nach links ( sozial $_{\text {). Mit der }}$ Integration der negativen Externalitäten und der Verschiebung des Gleichgewichts von Privat $Z$ Zu Psozial werden Schadenskosten in der Höhe $a+b$ reduziert. In diesem Fall reduziert sich die produzierte Menge auf qsozial und der Preis erhöht sich. Damit verbunden ist aber auch ein Rückgang der Konsumentenrente in der Höhe $b$ und der Produzentenrente in der Höhe $c$. Diese Verluste haben zur Folge, dass der Wohlfahrtgewinn im sozialen Optimum aus $a-c$ besteht. Eine Steuer s, welche die Grenzkosten der Produktion erhöht, würde die negative Externalität internalisieren (qsozial).

Hat die Landwirtschaft Anrecht auf negative Externalitäten und muss sie für eine Reduktion entschädigt werden oder hat die Gesellschaft Anrecht auf sauberes Trinkwasser und die Bäuerinnen und Bauern sowie die Konsumentinnen und Konsumenten müssen die Kosten für die Internalisierung tragen (Bromley und Hodge, 1990)?

Die Abgeltung von Umweltleistungen über Direktzahlungen können grob entlang der Dimensionen der Instrumente in drei Kategorien unterteilt werden. Inputorientierte Massnahmen beschreiben Instrumente, die den Einsatz von Produktionsfaktoren regeln, entsprechend der Dimension Input-Outcome-Beziehung. Ein Beispiel dafür sind die Ressourceneffizienzbeiträge. Die Landwirte erhalten eine Zahlung, wenn sie eine bestimmte Technologie, z.B. Schleppschlauchverfahren, oder ein bestimmtes Managementverfahren, z.B. schonende Bodenbearbeitung, einsetzen. Outcomeorientierte oder resultatorientierte Massnahmen zielen nicht auf das Management der Landwirte, sondern auf einen tatsächlich gemessenen Output. Das klassische Beispiel sind die Biodiversitätsförderbeiträge der Qualität II. Die Betriebe erhalten diese Zahlung nur dann, wenn sie einen bestimmten Indikator für Biodiversität, z.B. seltene Arten, auf ihren Feldern tatsächlich nachweisen können. Projektorientierte Massnahmen zielen auf die Erbringung einer Leistung basierend auf überbetrieblicher Zusammenarbeit $a b$, entsprechend der Dimension Partizipation. Beispiele aus der Schweizer Landwirtschaft sind die Vernetzungsbeiträge oder Landschaftsqualitätsbeiträge, die der Landwirt nur erhält, wenn er sich in einem Projekt einsetzt. Aus der Definition der Massnahmekategorien wird klar, dass die einzelnen Instrumente sich in Bezug auf ihr Targeting und Tailoring und damit ihre konkrete Umsetzung unterscheiden. 
Im Kontext der inputorientierten Massnahmen wird oft mit einem sogenannten Preis-Standard-Ansatz operiert (Rieder und Anwander Phan-huy, 1994). Die Idee dieses Ansatzes ist, dass ein Standard für die Agrarumweltleistung definiert und der dazugehörige Preis bzw. die Direktzahlung so festgelegt wird, dass dieser Standard erreicht wird. Mithilfe des Trial-and-Error-Prinzips wird anschliessend das optimale Level der entsprechenden Leistung angesteuert. Der Vorteil ist, dass der monetäre Wert einer positiven Externalität oder die Vermeidungskosten einer negativen Externalität nicht a priori bekannt sein müssen. Das ist insbesondere dann wichtig, wenn der Wert schwer zu bestimmen ist, wie beispielsweise im Fall der Biodiversität. Das Schweizer Direktzahlungssystem basiert in vielen Bereichen auf diesem PreisStandard-Ansatz. Ursprünglich wurde das Niveau für die Zahlungen aufgrund von Opportunitätskostenüberlegungen und den aus dem Decoupling hervorgegangenen Einkommenseinbussen auf der Marktseite festgelegt. Dieses Niveau wurde anschliessend, in Abhängigkeit der Zielerreichung, im Rahmen der Agrarreformen kontinuierlich angepasst. So wurden beispielsweise die allgemeinen Direktzahlungen von ursprünglich 1500 Franken auf 1100 Franken gesenkt. Mit der Umwandlung dieser allgemeinen Direktzahlungen in den Versorgungssicherheitsbeitrag in der AP 14-17 wurde dieser Beitrag auf 900 Franken verringert. Für strategisch wichtige Kulturen, z.B. Raps, hingegen wurde der Einzelkulturbeitrag im gleichen Zeitraum erhöht. Auch die Beiträge für ökologische Ausgleichsflächen, die in Biodiversitätsförderflächenbeiträge (BFF) umgewandelt wurden, werden laufend angepasst (letztmals wurden die BFF der ersten Qualitätsstufe 2017 gesenkt). Evaluationen von inputorientierte Massnahmen zeigen, dass das Instrument von den Landwirten relativ gut akzeptiert wird, aber leider in den meisten Fällen nicht sehr effektiv ist (Pe'er et al., 2014; Pe'er et al., 2019).

Im Gegensatz zu den inputorientierten Massnahmen, die ein bestimmtes, meist extensives Management oder eine bestimmte Produktionstechnologie, z.B. Schleppschlauchverfahren, voraussetzen, sind resultatorientierte Massnahmen darauf ausgerichtet, die eigentliche ökologische Leistung einer eingesetzten Technologie oder eines Managementverfahrens abzugelten (Burton und Schwarz, 2013; Stolze et al., 2015). Mit Blick auf das Evaluationsschema von Agrarpolitiken heisst das, dass man den Output überspringt und direkt auf den Outcome abzielt. Das bedeutet auch, dass die Beurteilung der Kosteneffizienz in den Hintergrund rückt und nur die Kosteneffektivität beurteilt werden kann.

Das Beispiel aus der Schweizer Agrarpolitik für resultatorientierte Massnahmen sind die BFF der Qualitätsstufe II. Diese werden den Landwirten nur dann ausbezahlt, wenn gewisse seltene Pflanzen und eine vorgegebene Anzahl von unterschiedlichen Arten auf einer Fläche tatsächlich vorkommen. Das bedeutet, dass für die Bereitstellung von Biodiversität nicht mehr nur das Management entscheidend ist, d.h., wie oft 
die Wiese geschnitten wird und ob Nährstoffe eingebracht werden oder nicht, sondern auch, ob die Wiese tatsächlich einen Beitrag zur Biodiversität leistet. Der Vorteil dieser Massnahmen ist offensichtlich. Sie ist viel stärker zielorientiert (targeted). Es gibt keine Unsicherheit, ob die Massnahme tatsächlich einen zusätzlichen Beitrag zur Biodiversität leistet oder nicht (Additivität). Der Nachteil ist, dass das Vorhandensein der Biodiversität überprüft und kontrolliert werden muss. Damit verbunden sind im Vergleich zu den inputorientierten Massnahmen höhere administrative Kosten. Auch bestehen grosse Unterschiede in den verschiedenen Produktionsregionen der Schweizer Landwirtschaft. In Gebieten, die in der Vergangenheit von intensiver Nutzung und hohem Tierbesatz geprägt waren, kann die fehlende Biodiversität nicht sofort mit einer einfachen Anpassung des Managements erreicht werden, weil es unter Umständen Jahre dauert, bis sich die Nährstoffkonzentration in den Böden so weit verringert hat, dass sich die Artenzahl tatsächlich erhöht. Damit ist auch der Verteilungswirkung der Massnahmen geringer. Eine geringere Anzahl von Bäuerinnen und Bauern können daher überhaupt von BFF der Qualitätsstufe II profitieren. Diese Nachteile, hohe administrative Kosten und der Einfluss von zeitlichen und räumlichen Einflussfaktoren ausserhalb der Landwirtschaft liegend, machen resultatorientierte Massnahmen weniger lukrativ für die Landwirtschaft als inputorientierte Instrumente. Aus einer ökologischen Perspektive wären sie aber der Goldstandard der Agrarumweltpolitik.

Eine weitere Form von Direktzahlungen in der Agrarumweltpolitik bilden jene Instrumente, die ein kollektives Handeln der Landwirte voraussetzen (Prager, 2015; Kuhfuss et al., 2016a). Gewisse Ziele der Umweltpolitik, wie beispielsweise die Pflege einer attraktiven Landschaft, können nicht auf einer einzelbetrieblichen Ebene gesteuert werden, weil nur die Gesamtheit der individuellen Entscheidungen zum Bild einer Landschaft führt. Das Beispiel für solche kollektiven Massnahmen sind in der Schweiz die Landschaftsqualitätsbeiträge. Diese sind projektbasiert und werden nur an diejenigen Betriebe ausgerichtet, die sich aktiv bei der Entwicklung von Zielen und dazugehörigen resultatorientierten Massnahmen einbringen. Innerhalb des Projekts können inputorientierte oder resultatorientierte Instrumente zur Anwendung kommen. Ein Beispiel für inputorientierte Massnahmen mit kollektiven Vorgaben sind in der Schweiz die Vernetzungsbeiträge für die räumlich koordinierte Anlegung von BFF. Das grosse Potenzial dieser Instrumente ist, dass sie ein Ziel abgelten, dass über den einzelnen Betrieb hinaus definiert ist, wie Landschaft und Biodiversität. Grundsätzlich liesse sich mit solchen projektbezogenen Instrumenten die unterschiedlichen Erbringungskosten bzw. Schadensverminderungskosten der Betriebe stärker ausnutzen. Damit können die gleichen Umweltziele mit weniger Kosten erbracht werden (siehe nächster Abschnitt). Mit Blick auf die Effizienz sind daher kollektive Massnahmen ein geeignetes Element. Dies trifft allerdings nur dann zu, wenn mit dem entsprechenden Projekt auch eine additive Wirkung verbunden ist. Diesbezüglich hat eine Evaluation der Landschaftsqualitätsbeiträge gezeigt (Jenny et al., 2018), dass in vielen Projekten 
Ziele und Massnahmen so festgelegt wurden, dass die Landwirte ihre Produktionsaktivitäten gar nicht anpassen mussten (Mitnahmeeffekte).

\subsubsection{Versteigerung von Umweltleistungen}

Die Kombination von resultatorientierten und projektbezogenen Instrumenten hätten somit das Potenzial, die Agrarumweltpolitik effektiv und effizient zu lenken. Das Problem ist, dass der Regulator, d.h. der Principle, normalerweise die effektiven Kosten der Landwirte, d.h. der Agenten, zur Erbringung einer Leistung, d.h. die Opportunitätskosten, nicht kennt. Diese sogenannte Principle-Agent-Problematik führt dazu, dass Leistungen entweder nicht ausreichend oder nur zu hohen Kosten erbracht werden (Hendrikse, 2003). Der grundlegende Mechanismus liegt darin begründet, dass die Kosten zur Erbringung einer Umweltleistung auf jedem landwirtschaftlichen Betrieb unterschiedlich sind. Legt der Regulator ein Ziel fest, z.B. 7 \% BFF auf jedem Betrieb, dann müssen auch diejenigen Betriebe mit hohen Opportunitätskosten diese ausscheiden. Würde der Regulator einen einheitlichen Preis (= Direktzahlung) für sämtliche Betriebe festlegen, dann wird derjenige Betrieb mit tiefen Opportunitätskosten automatisch mehr vom Umweltgut bereitstellen. Derjenige Betrieb mit hohen Opportunitätskosten dagegen würde weniger BFF ausscheiden. Die Festlegung des Preises anstelle einer fixen Menge führt theoretisch dazu, dass die unterschiedlichen Grenzkosten der Erbringung zwischen den Betrieben berücksichtigt werden können und damit eine kosteneffizientere Erbringung der Leistungen garantiert wird (Fraser, 2009). Ohne Fixierung der gewünschten Menge des Umweltguts besteht aber die Gefahr, dass die Landwirte zu wenig BFF anbieten. Der Preis-Standard-Ansatz versucht diese Problematik mit iterativen Schritten zu umgehen. Trotzdem werden auch mit diesem Instrument die unterschiedlichen Erbringungskosten der Betriebe nicht berücksichtigt.

Der agrarökonomische Ansatz zur Lösung für dieses Problem ist das Versteigerungsverfahren (Auktion) (Ferraro, 2008; Hanley et al., 2012; Lundberg et al., 2018). In einer Auktion würde der Regulator, d.h. das BLW, festlegen, wie viele BFF er in einer Region haben möchte. Diese Menge würde ausgeschrieben und die Landwirte würden einen Preis (= die Höhe der Direktzahlung) bieten. Anschliessend könnte das BLW aus den Geboten auswählen, sodass das Umweltziel zu den tiefsten angebotenen Preisen erbracht wird. Man spricht in diesem Fall von einem Preiswettbewerb (Höchli et al., 2009). Das heisst, dass die Landwirte selbst entscheiden müssten, zu welchem Preis sie bereit wären, die ökologische Leistung zu erbringen. Da die Landwirte untereinander in Konkurrenz stehen, kann auf diese Weise ein Teil der Produzentenrente abgeschöpft werden, ohne dass der Verwaltung Kosten für die Informationsbeschaffung entstehen. Ein Preiswettbewerb ist vor allem dann sinnvoll, wenn die Landwirte unterschiedliche Erbringungskosten haben, die Umweltleistung aber tendenziell homogen ist. Eine andere Form der Versteigerung ist der Leistungswett- 
bewerb (Höchli et al., 2009). In diesem wird ein Preis bzw. eine Direktzahlung festgelegt und die Landwirte bieten, welche Umweltleistung sie für diesen Preis anbieten würden. Der Leistungswettbewerb ist sinnvoll, wenn es grosse Unterschiede in Bezug auf die Umweltleistung gibt, die Erbringungskosten jedoch eher homogen sind. Ist zu erwarten, dass jeder Landwirt die gleiche Leistung erbringen kann (Leistungshomogenität) und dass die Erbringungskosten der Landwirte tendenziell ähnlich sind (Homogenität der Kosten), dann sind Ausschreibungen oder Versteigerungen ein ungeeignetes agrarpolitisches Instrument. Ohne Konkurrenz zwischen den Landwirten funktioniert eine Ausschreibung nicht.

Aus Sicht des Regulators wäre eine Auktion dasjenige Instrument, mit welchem er die Höhe der Umweltleistung pro eingesetzten Franken maximieren könnte. An dieser Stelle offenbaren sich allerdings die Nachteile dieses Instruments. 1) Es bleibt unbegründet, weshalb Landwirte mit Umweltleistungen nicht auch Gewinne erzielen dürfen (Mann, 2006a). Im Markt werden ebenfalls Erlöse erzielt, die über den Gestehungskosten zu liegen kommen. Nahrungsmittel sind da keine Ausnahme (Hirsch, 2018). 2) Durch die Auktion entstehen, je nach Ausgestaltung, administrative Kosten, welche die Wohlfahrtseffekte schmälern können. Dabei stellt sich insbesondere die Frage, wie gut die Landwirte überhaupt ihre Opportunitätskosten kennen. Eine Untersuchung aus Deutschland beispielsweise zeigte, dass die Gebote der Landwirte überhaupt nicht mit den von Wissenschaftlern berechneten Kosten übereinstimmten (Ulber et al., 2011).

\subsubsection{Instrumente der Einkommenspolitik}

Die Instrumente der Einkommenspolitik gründen auf den Überlegungen des Decouplings. Zur Erinnerung: Das Decoupling geht davon aus, dass die Unterstützung der Einkommen in der Landwirtschaft von der Preispolitik im Agrarsektor getrennt werden kann (OECD, 2006).

Diese Trennung kann aber unterschiedlich ausgestaltet werden. In der Schweiz wurde die Reduktion des Grenzschutzes mit Flächenzahlungen (allgemeinen Direktzahlungen) kompensiert. Dies hat den Vorteil, dass nach wie vor ein klarer Bezug zur Landwirtschaft besteht, ohne dass die Produktionsmenge zu stark beeinflusst wird. Ein Nachteil dieser Kompensation ist, dass sich die Zahlung auf den Bodenmarkt auswirkt und den Strukturwandel in der Landwirtschaft möglicherweise verlangsamt (Graubner, 2017). Alternativ kann die Einkommenspolitik auch über Betriebsbeiträge erfolgen. Diese sind vollkommen von der Produktion entkoppelt. Der Nachteil ist aber offensichtlich, dass dadurch auch hier der Strukturwandel beeinflusst wird (Mittenzwei und Britz, 2018). Je nach Ausgestaltung des Instruments kann dieser beschleunigt oder verlangsamt werden. Betriebsbeiträge wurden in der Schweiz Ende der 1980er-Jahre für Bergbetriebe eingesetzt. Die strukturhemmende Wirkung der damaligen Beiträge führte aber dazu, dass sie im Rahmen der Neuausrichtung der 
Agrarpolitik am Ende des letzten Jahrhunderts wieder aufgegeben wurden. Eine dritte Variante der Einkommenspolitik wäre eine Einkommensversicherung (Finger und El Benni, 2014). Die Landwirte könnten eine Versicherung abschliessen, die in Jahren mit tiefen Einkommen eine Kompensation ermöglicht. Werden die Prämien für diese Versicherung durch den Staat subventioniert, dann stützen diese Versicherungen die landwirtschaftlichen Einkommen. Der Vorteil wäre, dass das Einkommen der Landwirte nur dann gestützt wird, wenn es die Betriebe auch tatsächlich nötig haben. Ausserdem kann ein solches Instrument zu einem Ausgleich der Einkommensungleichheit innerhalb der Landwirtschaft führen. Die Umsetzung solcher Versicherungslösungen ist aber momentan in der Schweiz wenig sinnvoll, weil durch die Direktzahlungen und die Agrarstützung die Einkommensschwankungen gering sind und eine Prämie dadurch sehr teuer würde (Finger und El Benni, 2014).

Die Einkommensunterstützung in der Landwirtschaft hat aber immer auch eine ordnungspolitische Dimension. Wenn man die Landwirte nur unterstützt, weil sie arm sind, dann handelt es sich nicht mehr um Agrar-, sondern um Sozialpolitik. In der Sozialpolitik müssten aber andere Regeln in Bezug auf die Eligibilität von Zahlungen angewandt werden. Diese Haltung, die Agrarpolitik als Sozialpolitik zu verstehen, findet aber auch unter bäuerlichen Vertretern im Parlament wenig Rückhalt.

\section{ZUSAMMENFASSUNG}

Aufgrund der vielen Ziele, welche mit der Agrarpolitik angestrebt werden, existiert auch eine breite Palette und Instrumenten. Das agrarpolitische Instrumentarium lässt sich grob in drei Kategorien unterteilen: Command and Control, Informationsmassnahmen und ökonomische Anreize wie Steuern oder Subventionen. Für die Wirkung dieser Instrumente ist aber die konkrete Ausgestaltung von grosser Bedeutung. Diese Ausgestaltung kann mithilfe von sogenannten Dimensionen beschrieben werden. Die Dimensionen "Input-Outcome-Beziehung», "Standortabhängigkeit» und "Intertemporale Flexibilität» sind die Grundlagen für die Zielausrichtung eines Instruments, d.h. das Targeting. Die Dimensionen «Grad der Partizipation», "Verteilung der Kosten» und die "Standortabhängigkeit» beschreiben die Zuschneidung oder das Tailoring von agrarpolitischen Massnahmen. Daneben spielt es eine wichtige Rolle, bei wem der Ermessensspielraum liegt, beim Landwirt oder beim Staat, und ob Preise oder Mengen reguliert werden. Schliesslich spielen auch Informationen und Datenaustausch zwischen Staat und Landwirtschaft eine wichtige Rolle. Schliesslich lassen sich die agrarpolitischen Massnahmen auch aus einer wohlfahrtsökonomischen Perspektive beschreiben. Unter der Annahme von effizienten (Agrar-)Märkten führen Eingriffe in Angebot oder Nachfrage der landwirtschaftlichen Produktion zu Wohlfahrtsverlusten. Agrarumweltmassnahmen greifen über die Steuerung der Intensität oder der genutzten Flächen indirekt auch in die Agrarmärkte ein. Entscheidend für deren 
Wohlfahrtseffekt ist oft das ganze Paket der angewandten Instrumente. Für eine empirische Überprüfung der Effektivität und Effizienz von agrarpolitischen Massnahmen sind erweiterte ökonomische Modelle zwingend (Wettbewerbsform, Informationsasymmetrien, Heterogenität der Produktion, dynamische Effekte etc.). Die zentrale Frage ist dabei nicht, ob es Handel mit Agrarprodukten geben soll, sondern unter welchen Regeln dieser erfolgen soll. Die Evaluationen von agrarpolitischen Instrumenten in der Schweiz zeigen in vielen Fällen, dass die Effektivität (Zielerreichung) der Massnahmen hoch ist; die Effizienz und Verteilungswirkung aber oft kritisch beurteilt wird.

\section{WEITERFÜHRENDE LITERATUR}

Eine gute Zusammenfassung mit vielen weiterführenden Referenzen ist der Artikel von Sterner und Koautorinnen und Koautoren mit dem Titel «Policy design for the Anthropocene» in der Zeitschrift Nature Sustainability.

Der Artikel von Wunder und Koautorinnen und Koautoren ebenfalls aus der Zeitschrift Nature Sustainability mit dem Titel «From principles to practice in paying for nature's services» bietet einen interessanten und konzisen Überblick zu agrarpolitischen Massnahmen.

Die theoretischen Auswirkungen von Marktinterventionen werden ausführlich im Beitrag von Alston und James "The incidence of agricultural policy» im Handbook of Agricultural Economics beschrieben.

Eine interessante Perspektive auf die Agrarumweltpolitik bietet auch das Handbuch Ergebnisorientierte Massnahmen zur Förderung der Biodiversität in der Berglandwirtschaft von Matthias Stolze und Koautorinnen und Koautoren. Das Buch arbeitet die verschiedenen Herausforderungen der Agrarumweltpolitik an einem konkreten Beispiel ganzheitlich heraus.

Ein wichtiges Buch, das über eine agrarökonomische Perspektive hinausgeht und die Rolle von Kultur und Soziologie im Kontext von Agrarumweltmassnahmen beschreibt, ist das Buch The Good Farmer von Rob Burton, Jérémie Forney, Paul Stock und Lee-Ann Sutherland. Lesenswert! 


\section{Eine kurze Geschichte der Schweizer Agrarpolitik}

\section{READERS' GUIDE}

In diesem Kapitel wird in vier Unterkapiteln die historische Entwicklung der Agrarpolitik seit dem Ende des 19. Jahrhunderts vorgestellt. Das erste Kapitel beschreibt kurz die Agrarpolitik bis zum Ende des Zweiten Weltkriegs. Im zweiten Kapitel wird die aus einer politischen Sicht stabile Phase zwischen Kriegsende und dem Anfang der Neunzigerjahre erläutert. Diese mündete in einer dritten Phase, welche unter dem Begriff der Multifunktionalität der Landwirtschaft auch die heutige Agrarpolitik noch prägt. Abschliessend wird ein Schlaglicht auf die spezielle Rolle der Volksinitiativen mit Bezug zur Landwirtschaft geworfen und ein kurzer Ausblick auf die aktuellen Debatten geboten. Das Ziel dieses Kapitels ist es, dass die Leserinnen und Leser die historische Entwicklung der Agrarpolitik nachvollziehen können und die Phasen mit unterschiedlichen Schwerpunkten und Zielsystemen unterscheiden können.

Aufgrund der elementaren Bedeutung des Primärsektors für die wirtschaftliche und gesellschaftliche Entwicklung haben die Menschen seit jeher versucht, die Land- und Ernährungswirtschaft zu kontrollieren, regulieren und zu steuern (Rieder, 1993). Ausgehend von der Domestizierung der Nutztiere über die Etablierung der DreiFelder-Wirtschaft vor Tausenden von Jahren bis hin zur Grünen Revolution im letzten Jahrhundert und der heutigen Nutzung von Informations- und Kommunikationstechnologien war die Entwicklung von landwirtschaftlichen Produktionssystemen immer begleitet vom Willen, die wirtschaftlichen, rechtlichen und sozialen Verhältnisse der Landwirtschaft zu bestimmen. Die Regelung von Handelsströmen, Eigentumsrechten an Boden und Arbeitskräften sowie die Verfügbarkeit und Sicherstellung von Nahrungsmitteln waren Schlüsselelemente in der Entstehung und Entwicklung von $\mathrm{Na}$ tionen, Wirtschafts- und Gesellschaftssystemen (Diamond, 2014). Die Ursprünge der Agrarpolitik sind daher schon sehr alt und deren historische Entwicklung ein wichtiger Pfeiler für das Verständnis derer heutigen Ausgestaltung.

\subsection{Ursprung der heutigen Agrarpolitik}

Eine Agrarpolitik im heutigen Sinne, d.h. als sektorale Wirtschaftspolitik, welche Rahmenbedingungen für die Landwirtschaft schafft, existiert dagegen erst seit Ende 
des 19. Jahrhunderts (Fritzsche und Lemmenmeier, 1994; Haller, 2011). Die Industrialisierung und die damit verbundenen neuen Arbeits- und Produktionsverhältnisse und insbesondere die Möglichkeit, Nahrungsmittel über weite Strecken zu transportieren, führten dazu, dass sich die damaligen Nationen gezwungen sahen, die inländische Produktion vor billigeren Importen zu schützen und die Nahrungsmittelsicherheit der eigenen Bevölkerung durch Gesetze, welche die inländische Produktion stärkten, zu fördern. Zudem schwankten die Preise stark (Volatilität der Preise), wodurch die an die natürlichen Produktionszyklen gebundene Landwirtschaft mit grossen Unsicherheiten konfrontiert wurde. Das erste Gesetz für die Schweizer Landwirtschaft war der sogenannte Getreideartikel (1929), der den Import von Weizen regeln sollte (Popp, 2001).

Zu Beginn brachte die Industrialisierung auch der Landwirtschaft einen anhaltenden Produktivitätsschub. Im Laufe der Zeit entkoppelte sich jedoch das Wachstum des zweiten Sektors vom bodenabhängigen Produktionswachstum im Primärsektor. Die Landwirtschaft konnte die dadurch steigenden Löhne für Arbeitskräfte nicht mehr bezahlen und die familieneigenen Arbeitskräfte mussten fortan die landwirtschaftliche Produktion aufrechterhalten. Es folgte daher eine «Verbäuerlichung» der Landwirtschaft (Moser, 2006). Durch die zunehmenden Importe und dem daraus entstandenen Preisdruck wurde die landwirtschaftliche Produktion dennoch für viele der neu entstandenen Familienbetriebe unrentabel. Hohe Verschuldung und Armut prägten daher den Agrarsektor am Anfang des 20. Jahrhunderts. Die Erfahrungen aus dem Ersten Weltkrieg förderten den gesellschaftlichen Willen, die Landwirtschaft politisch zu stärken. Viele grundlegende Massnahmen wie beispielsweise die Unterstützung von landwirtschaftlichen Produzentenorganisationen, Investitionshilfen oder das Ertragswertprinzip stammen aus den 1920er- und 1930er-Jahren (Popp, 2001).

\subsection{Staatliche Ordnung nach den Weltkriegen}

Geprägt vom Zweiten Weltkrieg fokussierte die Agrarpolitik ab den 1950er-Jahren ganz auf die Produktionsziele der Landwirtschaft. Ernährungssicherheit, hohe Erträge und sichere Einkommen für die Bäuerinnen und Bauern waren prioritär (Rieder und Anwander Phan-huy, 1994). Grenzschutz und Abnahmegarantien durch parastaatliche Unternehmen wie die Käseunion oder Butyra sorgten dafür, dass die Produzentenpreise anstiegen und hoch blieben. Gleichzeitig fand in den entwickelten Ländern der westlichen Welt eine bis dahin ungesehene Ausdehnung der landwirtschaftlichen Produktion statt. Die sogenannte «Grüne Revolution» führte durch Mechanisierung und den Einsatz von Dünger und Pflanzenschutzmitteln zu immer effizienteren Produktionssystemen und der Abwanderung von Arbeitskräften aus der Landwirtschaft. Man bezeichnet diese Triebkräfte als Push-Faktoren des landwirtschaftlichen Strukturwandels. Parallel dazu benötigten der zweite und dritte Sektor aber auch stetig neue Arbeiter, um das Wachstum der Wirtschaft aufrechtzuerhalten. 


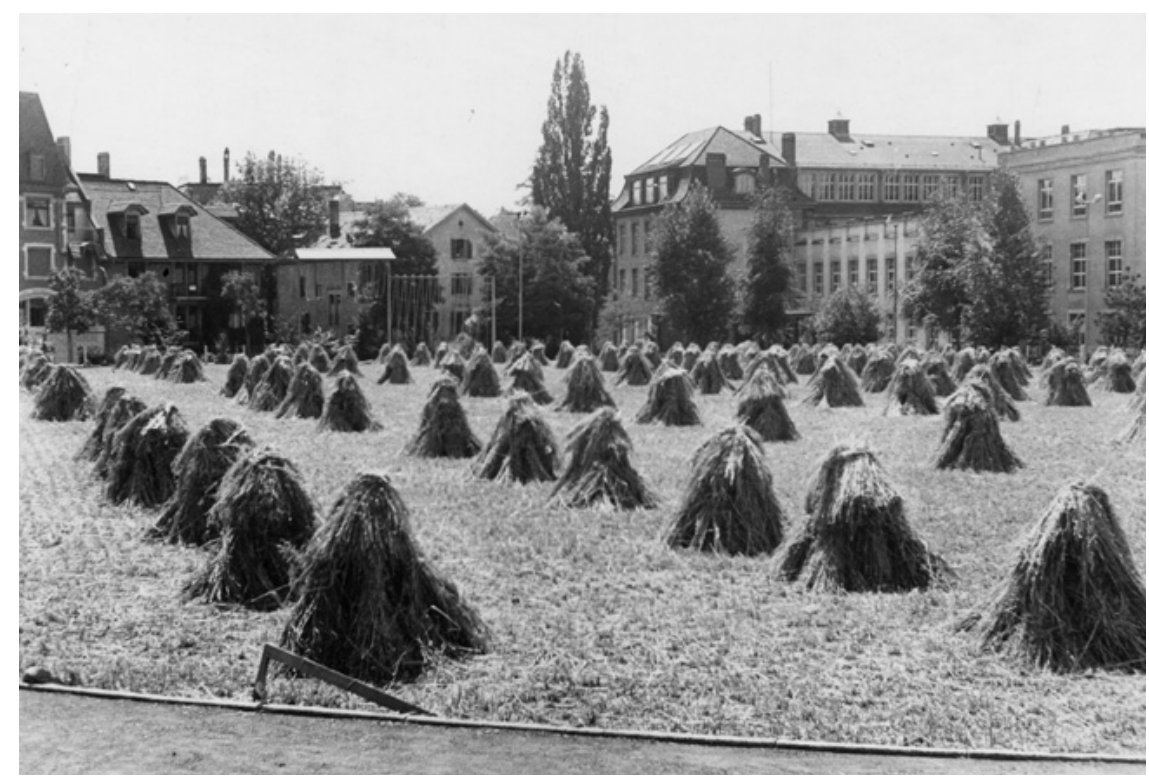

Abbildung 8: Nach den Weltkriegen standen die Ernährungssicherheit, hohe Erträge und sichere Einkommen für die Bäuerinnen und Bauern im Zentrum der Agrarpolitik. Im Bild die Turnwiese an der Rämistrasse in Zürich während der "Anbauschlacht». (Bild: Gallas Wilhelm, Baugeschichtliches Archiv Zürich)

Dies förderte ebenfalls die Abwanderung aus dem ersten Sektor und wird als Pull-Faktor des landwirtschaftlichen Strukturwandels bezeichnet (Popp, 2001). Zwischen 1940 und 1970 sank aufgrund dieser Push- und Pull-Faktoren die Anzahl der Landwirtschaftsbetriebe von 230000 auf weniger als 100000 Betriebe. Trotz dieses fundamentalen Wandels blieben die landwirtschaftlichen Einkommen tief. In Ländern, in welchen das volkswirtschaftliche Gewicht hoch blieb, war dies eher der Schwäche der übrigen Wirtschaftssektoren geschuldet als der eigentlichen Stärke der Landwirtschaft. Mit der anhaltenden Ausdehnung der landwirtschaftlichen Produktivität, also dem Verhältnis zwischen Input und Output und der Erhöhung der (Flächen-)Intensität, d.h. dem Kapitaleinsatz pro Fläche aufgrund der hohen Preise, produzierten die Bauern nicht nur zu viel (Butterberge, Milchseen), sondern es akzentuierten sich auch die Umweltprobleme einer intensiven Landwirtschaft. Der Bund zahlte Ende der 1980er-Jahre mehr als 2 Mia. Schweizer Franken für die Überschussverwertung der Schweizer Landwirtschaft, während gleichzeitig Lebensmittelskandale wie Nitrat im Kopfsalat die Konsumenten für die Lebensmittelsicherheit sensibilisierten (Haller, 2011). Gleichzeitig entstand internationaler Druck auf die Schweizer Landwirtschaft. Im Rahmen der achten Verhandlungsrunde des «General Agreement on Tariffs and Trade» (GATT) wurde von interna- 
tionaler Seite ein Ende der produktionsorientierten Stützung der Landwirtschaft gefordert. Diese sogenannte Uruguay-Runde endete mit der Gründung der WTO und neuen internationalen Regeln für die Agrarpolitik (Mann, 2003).

\subsection{Agrarpolitik für eine multifunktionale Landwirtschaft}

Neben dem internationalen Druck führten Überschüsse und Umweltprobleme auch zu gesellschaftlichem und politischem Druck innerhalb der Schweiz. Zwischen 1980 und 1998 fanden zehn eidgenössische Abstimmungen zur Landwirtschaft statt (Huber und Finger, 2019). Die Verwaltung - in enger Zusammenarbeit mit Forschenden - entwarf daraufhin den 7. Landwirtschaftsbericht, in welchem eine fundamentale Neuorientierung der Schweizer Landwirtschaftspolitik konzipiert wurde. Das wesentliche Element dieser Neuausrichtung war die Trennung der Preis- und Einkommenspolitik. Mit anderen Worten, die Einkommensproblematik der Landwirtschaft (siehe auch FarmProblem) sollte nicht mehr über möglichst hohe Produzentenpreise gelöst werden, sondern über produktionsunabhängige Direktzahlungen (Popp, 2001).

Diese Direktzahlungen sollten sich an einer multifunktionalen Landwirtschaft ausrichten. Das heisst, neben einer auf den Markt ausgerichteten Produktion soll die Landwirtschaft zu einer sicheren Versorgung der Bevölkerung, der Pflege der Kulturlandschaft und Erhaltung der natürlichen Ressourcen sowie zur dezentralen Besiedlung beitragen. Diese Ziele wurden 1996 mittels einer Volksabstimmung in der Schweizer Bundesverfassung im Artikel 104 verankert. Die Gewichtung der Produktionsziele der Agrargesetzgebung aus der Zeit nach dem Zweiten Weltkrieg wurde dadurch zugunsten von ökologischen und landschaftspflegerischen Zielen sowie der Nahrungsmittelsicherheit verringert.

Seit dem 21. Jahrhundert orientiert sich die Schweizer Agrarpolitik an diesem Verfassungsartikel. Da der Artikel auch vorsah, dass das Parlament alle vier Jahre über den Zahlungsrahmen für das Budget zur Erfüllung dieses Artikels abstimmt, ist die gesetzliche Umsetzung der Ziele einer ständigen Überprüfung und Anpassungsprozess unterworfen. Die Anpassungen wurden jeweils gebündelt und in einer sogenannten Agrarreformetappe (AP) umgesetzt (siehe Box 10). Die vier Weiterentwicklungsschritte der Schweizer Agrarpolitik in diesem Jahrhundert wurden immer mit AP und dem dazugehörigen Jahr der Einführung bezeichnet, d.h. AP02, AP07, AP11 und AP14-17. Aktuell ist die AP18-21 in Kraft, die am 7. März 2017 im Parlament verabschiedet wurde. Im September 2017 stimmte die Bevölkerung einer Ergänzung des Artikels 104 BV mit dem Artikel 104a BV zu. Letzterer dehnt die Ziele der Schweizer Agrarpolitik auf den Ernährungssektor, d.h. auf Aspekte der gesamten Wertschöpfungskette (ressourceneffiziente Lebensmittelproduktion) und der Konsumenten (ressourcenschonender Umgang mit Lebensmitteln) aus. Geplant ist, diesen Artikel in der nächsten Agrarreform umzusetzen. 
Box 10 Agrarreformetappen seit der Jahrtausendwende

\begin{tabular}{|c|c|c|c|c|c|}
\hline & AP 2002 & AP 2007 & AP 2011 & AP $14-17$ & AP $22+$ \\
\hline 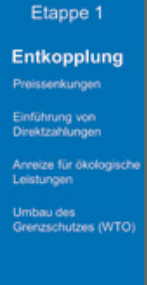 & 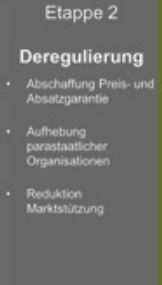 & 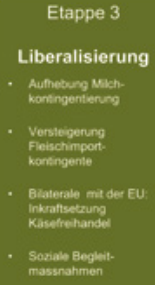 & 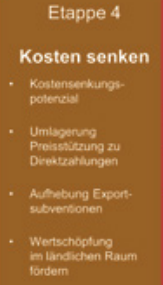 & 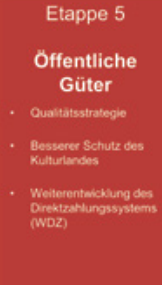 & 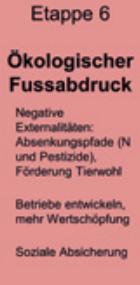 \\
\hline $1992-1998$ & $1999-2003$ & $2004-2007$ & $2008-2013$ & $2014-2023$ & 2023 - \\
\hline
\end{tabular}

1992 führte die Schweiz erstmals Direktzahlungen ein und leitete damit die Entkopplung der Preis- von der Einkommenspolitik ein. Mit der Annahme des Verfassungsartikels 1996 wurde die Schweizer Agrarpolitik konsequent auf die multifunktionalen Leistungen der Schweizer Landwirtschaft ausgerichtet. Dabei standen am Anfang die Abschaffung der Preisund Absatzgarantien und der Ausbau der Direktzahlungen im Vordergrund. Auch die parastaatlichen Unternehmen, welche für die Marktbereinigung zuständig waren, wurden mit der AP2002 aufgelöst. Die nächsten beiden Agrarreformetappen standen unter dem Zeichen der Liberalisierung und Deregulierung. Der schrittweise Abbau der Zölle mit der EU für Käse (2002-2007) war die wichtigste handelspolitische Öffnung der Schweizer Landwirtschaft. Zwei Jahre später, 2009, wurde die Milchkontingentierung abgeschafft. Auch im Bereich der Fleischproduktion wurde mit der Versteigerung der Importkontingente versucht, den Wettbewerb im Agrarsektor zu erhöhen. Zur Abfederung wurden verschiedene Begleitmassnahmen eingeführt wie beispielsweise die Verkäsungszulage oder die sozialen Begleitmassnahmen (siehe Kapitel 9). Die Agrarreformetappe 2011 versuchte dann den durch die Liberalisierung und Deregulierung ausgelösten Kostendruck auf die Landwirtschaft abzufedern. Dabei standen der Umbau von Marktstützungen in Direktzahlungen und die Förderung der Wertschöpfung im ländlichen Raum im Fokus. Die letzte umgesetzte Agrarreformetappe 14/17 war geprägt vom Umbau des Direktzahlungssystems. Mit der «Weiterentwicklung des Direktzahlungssystems (WDZ)» wurde die Stützung der Schweizer Landwirtschaft stringenter auf die Erbringung von öffentlichen Gütern ausgerichtet. Gleichzeitig wurden die Bemühungen zur Erhöhung der Wertschöpfung im Rahmen der sogenannten Qualitätsstrategie weitergeführt. 
Die AP22+ hatte ursprünglich eine Weiterentwicklung der Agrarpolitik in den Bereichen Unternehmertum der Betriebe, Wettbewerbsfähigkeit und den Schutz der natürlichen Ressourcen. Diese Ideen wurden aber im Verlauf der politischen Debatten und Auseinandersetzungen auf wenige Punkte wie beispielsweise die soziale Absicherung der Bäuerinnen reduziert. Erst durch den Druck von verschiedenen Volksinitiativen rückten Umweltthemen wieder in den Fokus der Agrarpolitik. Letztendlich wurde die Reform vom Parlament sistiert. Die Politik ist zurzeit daran, die Umsetzung einiger Elemente der AP22+ über den Verordnungsweg umzusetzen. Gleichzeitig soll eine neue Auslegeordnung die Grundlage für die nächste Agrarreformetappe legen.

\subsection{Bedeutung der Volksinitiativen in der Entwicklung der Agrar- politik}

Die Instrumente der direkten Demokratie, d.h. Referenda, Volksinitiativen und indirekte Gegenvorschläge spielten für die Entwicklung der Landwirtschaftspolitik immer wieder eine wichtige Rolle. Die Schweizer Bevölkerung befasste sich seit 1896 schon in 39 Abstimmungen direkt mit Gesetzes- oder Verfassungsänderungen die Landwirtschaft betreffend (Huber und Finger, 2019). Dabei lässt sich die Chronologie der Abstimmungen in vier Phasen unterscheiden (siehe Abbildung 9). Bis Ende der 1950er-Jahre wurde die entstehende Agrarpolitik nur durch wenige Abstimmungen beeinflusst. Mit einem neuen Wirtschaftsartikel in der Bundesverfassung wurde 1947 der Grundstein für eine neue Phase gelegt. Dieser Artikel legte die Handels- und Gewerbefreiheit in der ganzen Schweiz fest - definierte den Bauernstand und die Landwirtschaft aber als Ausnahme.

In der Folge war die Schweizer Landwirtschaftspolitik stark protektionistisch geprägt. Der Schutz und die Regulierung von Milch-, Getreide- und Zuckerproduktion wurden zwischen 1950 und 1985 von den Stimmbürgern in den meisten Fällen gestützt. Die Schweiz wurde dadurch zu einem der grössten Agrarprotektionisten weltweit. Wie im Kapitel 4.2 bereits vermerkt, stammten Ende der 1980er-Jahre von jedem Franken, den die Schweizer Bauern verdienten, 80 Rappen vom Staat. Die protektionistische Agrarpolitik kam Ende der 1980er-Jahre unter Druck. Gleichzeitig nahm auch die Unterstützung der Bevölkerung von protektionistischen Massnahmen ab. Die Ablehnung des Zuckerbeschlusses von 1986 leitete den Übergang in eine neue Phase ein. Erste Volksinitiativen wurden lanciert. Insbesondere die erste Kleinbauerninitiative (für ein naturnahes Bauern - gegen Tierfabriken), die nur mit 51,1 \% der Stimmen 
Eine kurze Geschichte der Schweizer Agrarpolitik

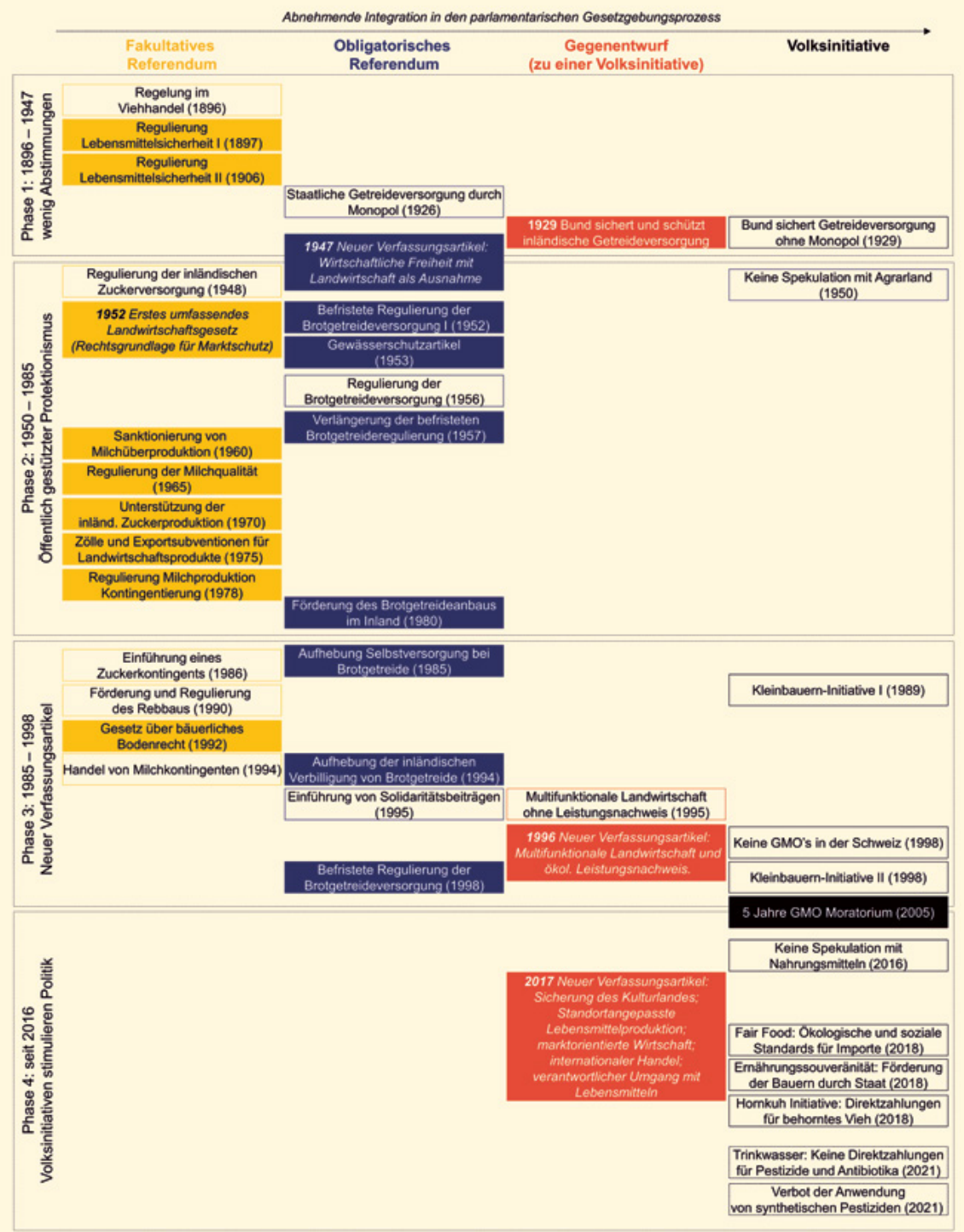

Abbildung 9. Chronologie der Abstimmungen zur Schweizer Landwirtschaftspolitik seit 1896. Eine farbig ausgefüllte Box bedeutet, dass die Vorlage angenommen wurde. Boxen ohne Farben bedeuten, dass die entsprechende Vorlage abgelehnt wurde. Quelle: in Anlehnung an Huber und Finger 2019. 
abgelehnt wurde, setzte die bestehende Agrarpolitik unter Druck. Ein erster Verfassungsartikel für eine multifunktionale Landwirtschaft ohne Einführung des ökologischen Ausgleichs wurde vom Stimmvolk jedoch abgelehnt (Haller, 2011). Erst die Verknüpfung der Direktzahlungen mit Umweltstandards verhalf dem bestehenden Artikel 104 BV zum Durchbruch. Diese führte zu einer Phase der relativen Stabilität.

In den letzten Jahren häuften sich nun aber die Volksinitiativen zur Landwirtschaft. Seit 2016 stimmten wir über sechs Initiativen (Keine Spekulation mit Nahrungsmitteln, Ernährungssouveränität, Fair Food, Hornkuh, Trinkwasser- und Pestizid) und einen Gegenvorschlag (Verfassungsartikel 104a) ab. Alle Initiativen wurden vom Stimmvolk verworfen. Auch die letzten Volksinitiativen, die Initiative für sauberes Trinkwasser und das Verbot von synthetischen Pestiziden, wurden vom Stimmvolk deutlich abgelehnt (Finger, 2021). In absehbarer Zeit wird das Stimmvolk auch über weitere Volksinitiativen mit direktem Bezug zur Landwirtschaft entscheiden (Massentierhaltungs-, Biodiversitäts- und Landschaftsinitiative).

Die Häufung von Initiativen ist sicher dem Umstand geschuldet, dass die Anzahl von Volksinitiativen generell, d.h. in allen Politikbereichen zunimmt. Darüber hinaus widerspiegeln die Abstimmungsthemen zwei zentrale Herausforderungen der Landwirtschaftspolitik: einerseits das Bestreben aus landwirtschaftlichen Kreisen, protektionistische Massnahmen (wieder-)einzuführen; andererseits der Anspruch, negative Umweltauswirkungen der Landwirtschaft zu reduzieren. Die hohen gesellschaftlichen Ansprüche an die Produktion von Lebensmitteln finden so Eingang in Initiativen, welche den bestehenden Kurs der Agrarpolitik ändern möchten. Die Volksinitiativen können daher als eine Art Barometer betrachtet werden, welches das Verhältnis zwischen den gesellschaftlichen Ansprüchen und deren Umsetzung im agrarpolitischen Prozess misst.

Auch wenn in der Vergangenheit elf von zwölf Volksinitiativen in der Agrarpolitik abgelehnt wurden, so wurden viele der neuen gesellschaftlichen Ansprüche in der Gesetzgebung, insbesondere über Gegenvorschläge, aufgenommen. Dies ermöglichte eine kontinuierliche Weiterentwicklung der Agrarpolitik.

\subsection{Anstehende Weiterentwicklung der Agrarpolitik}

Die Rolle der Volksinitiativen zeigt sich exemplarisch in der aktuellen Debatte zur nächsten Agrarreformetappe. Ursprünglich lag eine Vorlage vor, die eine kontinuierliche Entwicklung der bestehenden Reformschritte anvisierte. Die AP22+ stellte eine Art kleinster gemeinsamer Nenner zwischen den landwirtschaftlichen Kreisen, den Vertretern von Umwelt und Wirtschaft sowie der Verwaltung dar. Dieser Weg der kleinen Schritte kam jedoch durch zwei Entwicklungen unter Druck. Einerseits dräng- 
ten die parlamentarischen Vertreter der Landwirtschaft darauf, die Frage des Grenzschutzes und des hohen administrativen Aufwands stärker zu berücksichtigen. Andererseits drängte sich durch die Volksinitiativen, die in der Bevölkerung lange Zeit Sympathien genossen, eine stärkere Ausrichtung auf Umweltaspekte in den Vordergrund. Die AP22+ wurde in der politischen Debatte dadurch zum Symbol einer gescheiterten Agrarpolitik und das Parlament entschied aus verschiedenen Gründen, diese zu sistieren (siehe dazu auch Kapitel 10). Das agrarpolitische Vakuum, das dadurch entstand, wird in den nächsten Jahren auf drei Ebenen angegangen. Erstens wird die Regierung versuchen, gewisse Elemente der Agrarpolitik, welche im Parlament wenig umstritten waren, über den Verordnungsweg einführen. Dazu gehört beispielsweise die Verbesserung der sozialen Absicherung der Bäuerinnen, die in der AP22+ vorgesehen war. Zweitens wird, basierend auf einer parlamentarischen Initiative, ein Absenkpfad für Pestizide und Nährstoffverluste definiert, wodurch die Belastung von Oberflächengewässer, naturnahen Lebensräumen und des Grund- bzw. Trinkwassers reduziert werden soll. Erste Massnahmen sollen dazu 2023 umgesetzt werden. Schliesslich wird, drittens, die Verwaltung eine neue Auslegeordnung erarbeiten, die als Grundlage für die nächste Agrarreformetappe dienen soll. Basierend auf diesem Bericht wird die parlamentarische Diskussion über die nächsten Agrarreformschritte im Jahr 2023 wieder aufgenommen. Die Umsetzung dieser von der Verwaltung geplanten Schritte wird weiterhin durch Volksinitiativen beeinflusst werden wie beispielsweise die Massentierhaltungsinitiative, zu welcher der Bundesrat einen Gegenvorschlag entwickelt hat.

\section{ZUSAMMENFASSUNG}

Die heutige Agrarpolitik ist das Ergebnis einer fortlaufenden gesellschaftlichen, wirtschaftlichen und politischen Entwicklung über die letzten 150 Jahre. Während zu Beginn die Ernährungssicherheit der Bevölkerung und der Erhalt des Bauernstands im Fokus der Agrarpolitik lag, so entwickelte sich über die Zeit ein breiteres Zielspektrum der Agrarpolitik hin zu Umweltthemen, Handel und den Einbezug der gesamten Wertschöpfungskette bis hin zum Konsumenten. In den letzten Jahren entstanden viele Volksinitiativen, die auf der einen Seite das Unbehagen der Gesellschaft mit den nicht erreichten Umweltzielen ausdrückt und auf der anderen Seite die Bemühungen der Landwirtschaft zur Wiedereinführung von protektionistischen Massnahmen widerspiegelt. Dies führte zu einer Art "Marschhalt» in der Weiterentwicklung der Schweizer Agrarpolitik, welche seit der Einführung des neuen Verfassungsartikels 1996 auf fünf Agrarreformetappen aufbaute. Die nächsten Schritte in der Agrarpolitik sind erst im Jahr 2023 zu erwarten. 


\section{WEITERFÜHRENDE LITERATUR}

Interessante Literatur zur historischen Entwicklung der Agrarpolitik und Agrarwirtschaft in der Schweiz und Europa findet sich in den Schriften von Peter Moser, Leiter des Archivs für Agrargeschichte: www.histoirerurale.ch.

Ein konkretes Beispiel, wie die ökonomischen Kräfte schon sehr lange auf die Landwirtschaft und Bauern auswirkt, findet sich im Beitrag von Bruno Fritzsche und Max Lemmenmeier: "Die revolutionäre Umgestaltung von Wirtschaft, Gesellschaft und Staat 1780-1870». In: Geschichte des Kantons Zürich, Bd. 3, Zürich 1994, S. 20-43.

Peter Rieder, emeritierter Professor für Agrarökonomie an der ETH, hat seine Perspektive in den Ideen und Geistesgeschichte der Europäischen Agrarpolitik festgehalten.

Schliesslich bietet das Buch von Hans Popp Das Jahrhundert der Agrarrevolutionen. Schweizer Landwirtschaft und Agrarpolitik im 20. Jahrhundert eine chronologische Übersicht über die Agrarpolitik im letzten Jahrhundert. 


\section{Erscheinungsbild der Landwirtschaft in der Schweiz}

\section{READERS' GUIDE}

In diesem Kapitel wird das Erscheinungsbild der Schweizer Landwirtschaft kurz und knapp zusammengefasst. Die ldee ist, dass die Leserinnen und Leser Fakten über den Zustand und die Entwicklung der Schweizer Landwirtschaft kennenlernen. Das Kapitel ist in vier Themen unterteilt: 1) landwirtschaftliche Strukturen; 2) Produktion und Wertschöpfung; 3) Ausgaben des Bundes und der Konsumenten sowie 4) Umweltaspekte. Vielleicht besteht die Tendenz, dieses Kapitel zu überspringen, weil man glaubt, ohnehin schon viel über die Schweizer Landwirtschaft zu wissen. Trotzdem ist eine faktenbasierte Grundlage über den Zustand ein elementarer Bestandteil für das Verständnis der Agrargesetzgebung in der Schweiz und die agrarpolitischen Prozesse, die in den nächsten beiden Kapiteln behandelt werden. Das Ziel dieses Kapitels ist es, dass die Leserinnen und Leser ein paar wenige Fakten zur Landwirtschaft kennenlernen und diese, wenn notwendig, auch an dieser Stelle wieder nachschlagen können. Die Quelle für die folgenden Ausführungen ist der Agrarbericht des Bundesamts für Landwirtschaft (BLW diverse Jahrgänge). Dieser ist online frei zugänglich und ist für alle, die sich mit Agrarpolitik in der Schweiz beschäftigen, eine ausführliche und leserfreundliche Dokumentation der Schweizer Landwirtschaft und der Agrarpolitik.

\subsection{Landwirtschaftliche Struktur}

In der Schweiz gab es im Jahr 2020 rund 50000 Landwirtschaftsbetriebe und etwas weniger als 150000 Beschäftigte. Dies entspricht einem Anteil von knapp $\mathbf{3} \%$ der arbeitenden Bevölkerung. Im gesamten Lebensmittelbereich, d.h. in den vor- und nachgelagerten Betrieben der Landwirtschaft arbeiteten weitere 400000 Personen, davon ungefähr 120000 Beschäftigte in der Nahrungsmittelindustrie oder dem Grosshandel mit Nahrungsmitteln, ca. 100000 im Detailhandel und ca. 180000 in Restaurants oder Kantinen. Insgesamt sind in der Schweizer Lebensmittelkette ungefähr $11 \%$ der arbeitenden Bevölkerung beschäftigt. 
Im Durchschnitt bewirtschaften die Schweizer Landwirtschaftsbetriebe eine Fläche von 21 ha. Die Anzahl der Betriebe nahm im jährlichen Mittel der letzten fünf Jahre um 1,5\% ab. Kleinere Betriebe mit weniger als 10-20 ha nehmen überproportional ab. Die Anzahl der Betriebe mit mehr als 50 ha nimmt dagegen deutlich zu. Im Mittel nimmt dadurch auch die Betriebsgrösse über die Zeit zu. 1975 gab es noch rund 110000 landwirtschaftliche Betriebe. Bis 1995 reduzierte sich die Anzahl Betriebe auf rund 80000 . Die Geschwindigkeit, mit der Betriebe aufgegeben werden, nimmt in den letzten Jahren - in absoluten Zahlen gemessen - nicht zu.

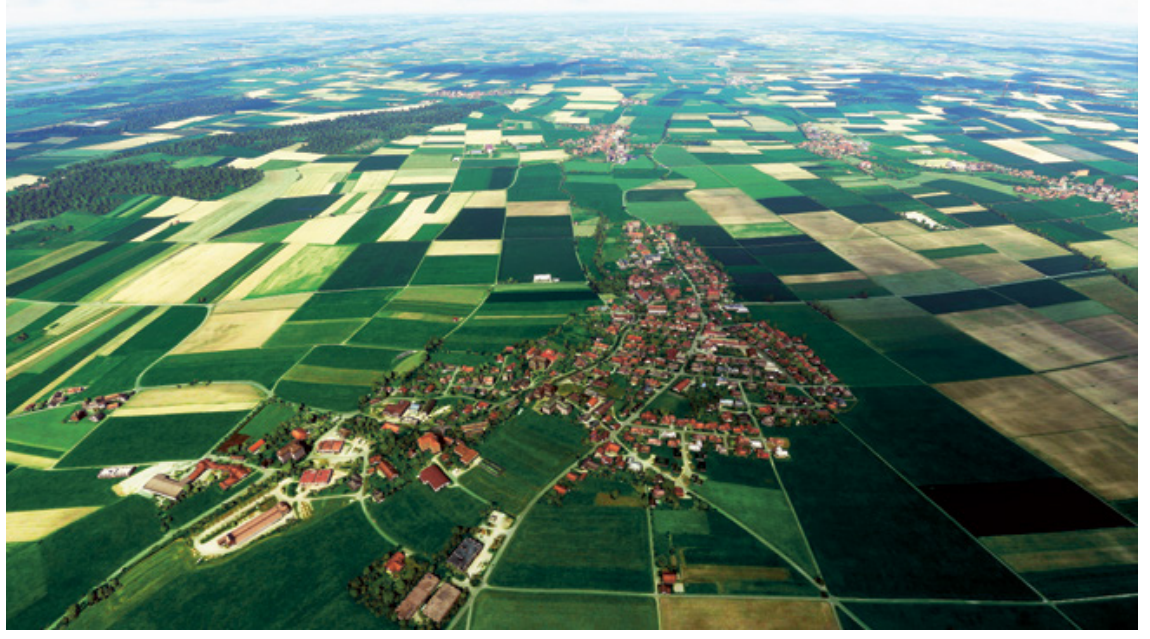

Abbildung 10. Die Schweizer Landwirtschaft ist im europäischen Vergleich kleinstrukturiert. Die Betriebe bewirtschaften im Durchschnitt etwas mehr als 20 ha landwirtschaftliche Nutzfläche. Damit verbunden ist eine kleinteilige Flächennutzung, welche das Landschaftsbild der Schweiz prägt (Bild: Vonkara/iStock).

\subsection{Produktion und Wertschöpfung}

Im Mittel der letzten Jahre produzierten die Landwirte in der Schweiz Produkte und Leistungen im Umfang von mehr als 11 Mia. CHF. Dies entspricht ungefähr 0,7 \% der gesamten Wertschöpfung in der Schweiz. Nach Abzug der Vorleistungen und der Abschreibungen erzielten die Betriebe im Jahr 2020 eine Nettowertschöpfung von rund 2,4 Mia. CHF. Die Landwirtschaft wird dabei jährlich mit rund 3,6 Mia. CHF durch den Bund unterstützt. Zählt man die Unterstützung ausserhalb des Zahlungsrahmens und die Ausgaben der Kantone hinzu, dann beläuft sich die jährliche totale Stützung auf etwas über 4 Mia. CHF (Dümmler und Roten, 2018). Das Faktoreinkommen, d.h. die Entschädigung für Arbeit, Kapital und Boden, der Schweizer Landwirtschaft umfasst damit etwas weniger als 5 Mia. CHF. Wenn man die Löhne für die landwirtschaft- 
lichen Angestellten und die Zinsleistungen abzieht, bleiben für das Selbstständigeneinkommen, das sogenannte Nettounternehmenseinkommen der Schweizer Landwirtschaftsbetriebe, rund 3 Mia. CHF bzw. 66000 CHF pro Betrieb. Das bedeutet, dass das Einkommen der Bäuerinnen und Bauern in der Schweiz tiefer ist als die landwirtschaftliche Stützung durch den Staat.

Pro Betrieb betrug das landwirtschaftliche Einkommen im Jahr 2020 rund 79000 CHF. Da im Normalfall mehr als nur eine Person auf einem Betrieb arbeitet, liegt der Arbeitsverdienst je Vollzeit-Familienarbeitskraft bei 58600 CHF. Dazu kommen Nebenerwerbseinkommen, welche das Haushaltseinkommen (oder Gesamteinkommen) um rund $30 \%$ erhöht. Das bedeutet, dass das Nebeneinkommen eine äusserst wichtige Bedeutung für die Bauernfamilien in der Schweiz hat. Es muss aber festgehalten werden, dass es grosse Unterschiede in den verschiedenen Regionen der Schweiz gibt. In der Talregion liegt das landwirtschaftliche Einkommen bei $92000 \mathrm{CHF}$ und das Gesamteinkommen im Durchschnitt bei $128000 \mathrm{CHF}$. In der Hügelregion liegen landwirtschaftliches und Haushaltseinkommen rund $20 \%$ tiefer. In der Bergregion liegt das mittlere Haushaltseinkommen bei knapp $88000 \mathrm{CHF}$ und das landwirtschaftliche Einkommen bei rund 58000 CHF. Ausserdem können Wetterbedingungen und Marktentwicklungen zu jährlichen Schwankungen im landwirtschaftlichen Einkommen führen. Schliesslich muss auch festgehalten werden, dass in der Landwirtschaft hohe Arbeitszeiten die Regel sind. Die wöchentliche Normalarbeitszeit liegt bei $\mathbf{6 0}$ Stunden und gehört damit zu den höchsten in allen Wirtschaftsbranchen.

Insgesamt wird eine landwirtschaftliche Nutzfläche (LN) von ca. 1 Mio. Hektaren (ha) bewirtschaftet. Das entspricht etwas mehr als $23 \%$ der Landesfläche. Rund $70 \%$ der LN wird als Grünfläche bewirtschaftet (Naturwiesen, Weiden, Kunstwiesen). Die Flächen, die sich für die Produktion von Ackerkulturen eignen und daher eine wichtige Grundlage für die Versorgungsbasis mit Nahrungsmitteln in Krisenzeiten darstellen sogenannte Fruchtfolgeflächen -, werden auf etwa $\mathbf{4 3 0} \mathbf{0 0 0}$ ha geschätzt. Tatsächlich bewirtschaftet werden $14 \%$ der LN mit Getreide, ca. $3 \%$ mit Kartoffeln, Zucker und Futterrüben, $3 \%$ mit Ölsaaten und weitere $11 \%$ mit Spezialkulturen und anderen Ackerfrüchten. Zur eigentlichen LN kommen noch Sömmerungsflächen, d.h. Alpweiden von ungefähr 400000 ha dazu. Die LN pro Person beträgt in der Schweiz etwas mehr als $1200 \mathrm{~m}^{2}$. Dies widerspiegelt die hohe Bevölkerungsdichte in der Schweiz. Im europäischen Vergleich weist nur die Niederlande eine ähnlich tiefe LN pro Person auf. In Deutschland liegt dieser Vergleichswert bei $2000 \mathrm{~m}^{2}$, in Frankreich bei mehr als $4000 \mathrm{~m}^{2}$. In diesen Ländern ist die Möglichkeit, die Bevölkerung mit eigenen Lebensmitteln zu versorgen, entsprechend höher. 
Entsprechend den Flächen sind auch die Betriebe wirtschaftlich ausgerichtet. Der überwiegende Anteil der Landwirtschaft produziert tierische Produkte (75 \%). Nur rund 10000 Betriebe fokussieren auf pflanzliche Produktion. Der Anteil der biologisch geführten Betriebe hat in den letzten Jahren stark zugenommen. Er beträgt mittlerweile knapp $15 \%$ der Betriebe und ungefähr $17 \%$ der Fläche. Die Anzahl der von Frauen geführten Betriebe lag 2020 bei 3233 Betrieben.

In den Betrieben mit Tierhaltung werden rund 1,5 Mio. Rinder gehalten. Davon sind es ungefähr $\mathbf{7 0 0} \mathbf{0 0 0}$ Milchkühe. Dieser Rindviehbestand hat sich in den letzten zehn Jahren um 8 \% verringert. Dazu kommen ca. 1,4 Mio. Schweine, 12,4 Mio. Hühner und knapp über 500000 Pferde, Schafe und Ziegen. Hühner, Schweine und Pferde werden vorwiegend im Tal gehalten. Schafe und Ziegen dagegen mehrheitlich im Berggebiet.

Die Wertschöpfung der landwirtschaftlichen Produktion ist ungefähr proportional der Flächennutzung. Der Wert der Pflanzenproduktion liegt bei ungefähr 4 Mia. CHF, derjenige der tierischen Produktion bei knapp $6 \mathrm{Mia}$. CHF pro Jahr. Es gilt allerdings zu beachten, dass dieser Produktionswert stark vom Schweizer Grenzschutz, d.h. den Zöllen und Zollkontingenten für landwirtschaftliche Produkte, geprägt ist. Die Produzenten- und Konsumentenpreise sind in der Schweiz wesentlich höher als im benachbarten Ausland. Nahrungsmittel sind im Schnitt $\mathbf{6 0} \%$ teurer als im Mittel der EU-15-Staaten.

Im Kontext der Wertschöpfungskette sind drei weitere Charakteristika der Schweizer Landwirtschaft erwähnenswert: 1) Die Wertschöpfungskette der Schweizer Nahrungsmittelproduktion ist durch eine sogenannte X-Struktur charakterisiert (siehe Kapitel 7.3). Das bedeutet, dass die Anzahl der Marktteilnehmer auf Stufe Produktion und Konsum hoch ist und auf der Stufe der Verarbeitung und des Handels - in der Mitte der Wertschöpfungskette - tief ist. Die beiden grössten Detailhändler Migros und Coop decken ca. 70 \% des Lebensmittelhandels in der Schweiz ab. Viele dieser Unternehmen verfügen über eine vertikale Integration innerhalb der Wertschöpfungskette. Das heisst, die Unternehmen unterhalten Industriebetriebe, welche auch die Lagerung und Verarbeitung von Nahrungsmittelrohstoffen abdecken. Ein wichtiges Unternehmen ist auch die Fenaco, die aus dem Zusammenschluss von Volg und landwirtschaftlichen Genossenschaftsverbänden entstand. Das Unternehmen deckt einen grossen Teil des Produktionsmittelmarktes in der Schweizer Landwirtschaft ab.

2) Der Anteil der landwirtschaftlichen Rohstoffe am Konsumentenpreis ist klein. Von der gesamten Food-Wertschöpfung, die mit den Konsumausgaben der Inländer ausgelöst wird, erwirtschaftet die Schweizer Landwirtschaft rund $7 \%$. Der Rest wird verteilt auf Handel, Transportwesen, die Nahrungs- und Genussmittelindustrie sowie importierte Vorleistungen und Fertigprodukte. 3) Während der Produktionspreisindex der landwirtschaftlichen Erzeugnisse in den letzten 15 Jahren rückläufig war (ca. $10 \%$ ), ist der Einkaufspreisindex für landwirtschaftliche Produktionsmittel im 
gleichen Zeitraum um 6 \% gestiegen. Das heisst, die Landwirtschaft verkauft zu immer tieferen Preisen und muss gleichzeitig die Inputs zu höheren Preisen einkaufen.

Der Selbstversorgungsgrad, d.h. der Anteil der im Inland produzierten Nahrungsmittel am Konsum der Bevölkerung in der Schweiz, liegt brutto bei knapp $60 \%$. Dieser Anteil ist seit den 1990er-Jahren mehr oder weniger konstant, obwohl die Bevölkerung in diesem Zeitraum angestiegen ist. Das bedeutet, dass $40 \%$ der Nahrungsmittel, die in der Schweiz konsumiert werden, importiert werden müssen. Allerdings unterscheidet sich der Selbstversorgungsgrad stark zwischen den unterschiedlichen Produkten. Bei tierischen Produkten liegt der Selbstversorgungsgrad bei knapp 100 \%. Für pflanzliche Produkte hingegen liegt dieser Wert lediglich bei $40 \%$. Zieht man davon die tierischen Produkte ab, welche auf der Basis von importierten Futtermitteln hergestellt werden, dann reduziert sich der Selbstversorgungsgrad um ca. 5$10 \%$. Der Netto-Selbstversorgungsgrad bei tierischen Produkten liegt bei $80 \%$ der im Inland produzierten Mengen und insgesamt bei einem Wert von ca. $\mathbf{5 0} \%$. Neben dem eigentlichen Selbstversorgungsgrad ist es auch wichtig, darauf hinzuweisen, dass die Schweizer Landwirtschaft viele Produktionsmittel als Vorleistungen importiert wie beispielsweise Kunstdünger, Saat- und Pflanzgut, Pflanzenschutzmittel, Treibstoffe oder Maschinen. Diese Vorleistungen machen rund $14 \%$ der gesamten Wertschöpfung im Food-Bereich aus.

\subsection{Ausgaben des Bundes und der Haushalte}

Die Ausgaben des Bundes für die Agrarpolitik lagen 2019 bei über 3,6 Mia. CHF. Das entspricht einem Anteil von $5 \%$ der gesamten Bundesausgaben. Der grösste Anteil dieser Ausgaben machen die Direktzahlungen aus, die sich auf rund 2,8 Mia. CHF belaufen. Daneben gibt der Bund rund 550 Mio. CHF für Massnahmen im Bereich Produktion und Absatz aus. Die Bundesausgaben sind seit Anfang der 1990er-Jahre auf einem ähnlichen Niveau. Allerdings hat sich das Verhältnis zwischen Direktzahlungen und den Ausgaben für Produktion und Absatz umgekehrt. 1990 lagen die Ausgaben für Produktion und Absatzmassnahmen noch bei über 1,5 Mia. CHF.

Ein wichtiger Aspekt in der Diskussion um die Schweizer Agrarpolitik sind auch die Haushaltsausgaben der Schweizer Konsumentinnen und Konsumenten. Im Schnitt betrugen im Jahr 2018 die direkten Ausgaben für Nahrungsmittel und alkoholfreie Getränke rund 6,3\% an den totalen Brutto-Haushaltseinkommen. Für Essen ausser Haus in Gast- und Beherbergungsstätten wurden weitere 4,7\% ausgegeben. Insgesamt werden im Schnitt pro Monat rund 1200 Franken oder $12 \%$ des Budgets für Ernährung ausgegeben. Im Vergleich: in Deutschland liegt der Anteil der Konsumausgaben für Nahrungsmittel bei knapp $20 \%$, in Frankreich bei $24 \%$ und in Italien bei $29 \%$ (allerdings inkl. Hotelausgaben). 


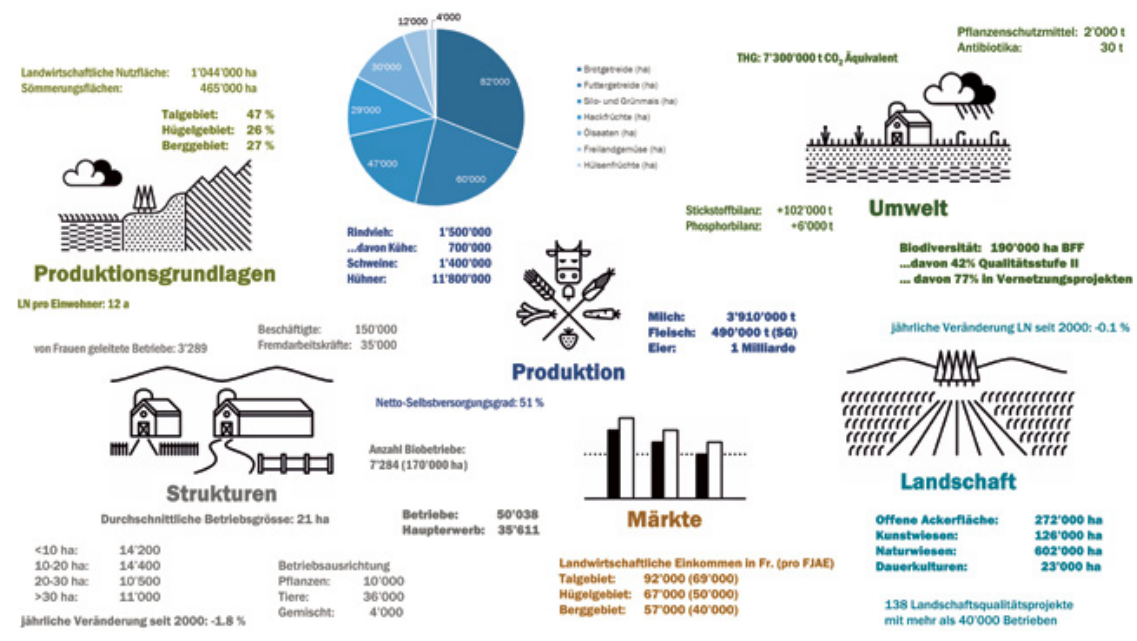

Abbildung 11. Erscheinungsbild Schweizer Landwirtschaft 2019 (Abkürzungen: FJAE: Arbeitsverdienst pro Familienarbeitskraft; BFF: Biodiversitätsförderflächen; ha: Hektare; LN: landwirtschaftliche Nutzfläche; SG: Schlachtgewicht).

\subsection{Umweltaspekte}

Zentral in der Diskussion um die Agrarpolitik sind auch die negativen Umwelteinflüsse der Landwirtschaft. Die Stickstoffüberschüsse, d.h. Bilanz der Stickstoffmengen, die in landwirtschaftliche Böden gelangen und ihnen entzogen werden, belaufen sich auf 100000 t N pro Jahr. Gemäss Bilanzrechnung sind das rund $70 \mathrm{~kg} \mathrm{~N}$ pro ha. Dieser Wert ist über die letzten 20 Jahre mehr oder weniger konstant geblieben. Auch die Verkäufe von Pflanzenschutzmitteln haben sich in den letzten zehn Jahren nicht verändert. Die Nachfrage nach Lebensmitteln durch die Schweizer Bevölkerung verursacht ausserdem rund 15 Mio. t $\mathrm{CO}_{2}$-Äquivalente (inkl. Importe). Davon ist etwa ein Drittel direkt auf die inländische Landwirtschaft zurückzuführen. In den letzten Jahren nahmen extensiv genutzte Flächen, die zur Förderung der Biodiversität dienen, von ca. 100000 ha am Anfang des Jahrtausends auf über 190000 ha zu. Allerdings entsprechen diese Flächen nicht zwangsläufig der Qualität, welche eine nachhaltige Biodiversität in der Agrarlandschaft sicherstellen kann. 


\section{ZUSAMMENFASSUNG}

Die Schweizer Landwirtschaft ist klein strukturiert. Die 50000 landwirtschaftlichen Betriebe sind im Durchschnitt 20 ha gross. Die landwirtschaftliche Nutzfläche besteht zu $70 \%$ aus Grasland. Der Produktionswert der Landwirtschaft beträgt rund 10, das sektorale Einkommen der Landwirte 3 Mia. CHF. Die gesamten Ausgaben des Bundes für die Landwirtschaft belaufen sich auf etwas mehr als 3,5 Mia. CHF jährlich. Dazu kommen noch die Ausgaben der Kantone. Die landwirtschaftlichen Einkommen sind im Vergleich zur übrigen Wirtschaft tief. Sie sind in den letzten Jahren jedoch tendenziell angestiegen. Der Anteil der landwirtschaftlichen Wertschöpfung an den gesamten Lebensmittelaufwendungen ist klein. Auch die Haushaltsausgaben der Schweizerinnen und Schweizer für Lebensmittel ist gering. Die Landwirtschaft produziert negative Externalitäten, insbesondere Stickstoffüberschüsse, und verursacht durch eine intensive Produktion eine Reduktion von Biodiversität. Gleichzeitig nutzen und pflegen die Bäuerinnen und Bauern die Kulturlandschaft. Die Schweizer Landwirtschaft trägt mit der Produktion von knapp 55\% der konsumierten Nahrungsmittel zur Versorgung der inländischen Bevölkerung bei.

\section{WEITERFÜHRENDE DATENQUELLEN}

Agrarbericht

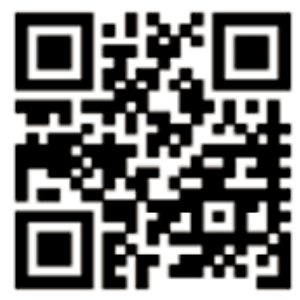

Statistische Erhebungen und Schätzungen SBV

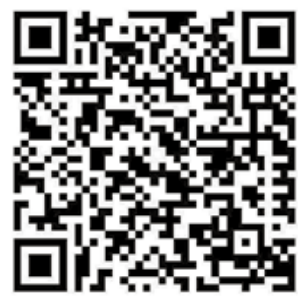

Bundesamt für Statistik

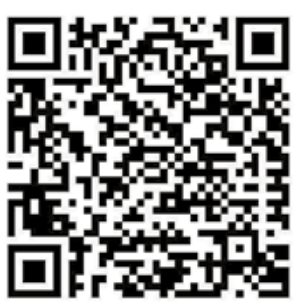




\section{Die Agrargesetzgebung in der Schweiz}

\section{READERS' GUIDE}

In diesem Kapitel wird die Agrargesetzgebung mit einem Fokus auf dem Landwirtschaftsgesetz sowie dem Bundesgesetz über das bäuerliche Bodenrecht vorgestellt. Die Quellen dieses Kapitels sind die Gesetze selbst (unter www.admin.ch abrufbar) sowie die Informationen des Bundesamts für Landwirtschaft (Webseite und Agrarbericht). Trotz der ausführlichen Beschreibung der Agrargesetzgebung in diesem Buch hat sie nicht den Anspruch, vollständig zu sein. Gesetze bedürfen immer auch einer Interpretation und manchmal müssen Gerichte entscheiden, wie ein bestimmter Paragraf auszulegen sei. In diesem Kontext publiziert das Bundesamt für Landwirtschaft immer auch aktualisierte "Weisungen und Erläuterungen» zu den eigenen Verordnungen. Diese erlauben es, die Logik der Behörde in der Umsetzung der Gesetze nachzuvollziehen. Es ist explizit nicht der Anspruch, an dieser Stelle sämtliche Weisungen und Erläuterungen hier aufzuführen. Das Ziel ist es jedoch, dass die Leserinnen und Leser einen Überblick über die geregelten Bereiche und das angewendete Instrumentarium erhalten. Dies soll es ermöglichen, die bestehenden Instrumente mit den theoretischen Kenntnissen der Agrarpolitik aus den Kapiteln 5 und 6 zu verbinden.

Die Grundlage der Schweizer Agrargesetzgebung sind die Artikel 104 und 104a der Bundesverfassung (Box 11). Basierend auf den Zielen und Leitplanken, welche in diesen beiden Artikeln festgehalten sind, konkretisieren das Landwirtschaftsgesetz (LwG), das Bundesgesetz über das bäuerliche Bodenrecht (BGBB), das landwirtschaftlichen Pachtgesetz (LPG) und weitere Formulierungen im Raumplanungs-, Umweltschutz-, Gewässerschutz- und Tierschutzgesetz die Schweizer Agrarpolitik.

Die Gesetze geben grundsätzlich vor, welche Ziele mit welchen Massnahmen erreicht werden sollen (für eine Darstellung der agrarpolitischen Ziele siehe Abschnitt 5.1). Die Gesetze setzen sich hierarchisch aus Titeln, Kapiteln, Abschnitten und Artikeln zusammen. 49 Verordnungen regeln die konkrete Umsetzung der Agrargesetzgebung. Die unterschiedlichen Verordnungen wie beispielsweise die landwirtschaftliche Begriffsverordnung (LBV), die Direktzahlungsverordnung (DZV) oder die Verordnung zur Strukturverbesserung in der Landwirtschaft (SVV) konkretisieren unterschiedliche Teilbereiche des Landwirtschaftsgesetzes. Diese werden von der Verwaltung verfügt. 


\section{Box 11 Bundesverfassung BV Art. 104 Landwirtschaft}

Der Bund sorgt dafür, dass die Landwirtschaft durch eine nachhaltige und auf den Markt ausgerichtete Produktion einen wesentlichen Beitrag leistet zur:

a. sicheren Versorgung der Bevölkerung;

b. Erhaltung der natürlichen Lebensgrundlagen und zur Pflege der Kulturlandschaft;

c. dezentralen Besiedlung des Landes.

Ergänzend zur zumutbaren Selbsthilfe der Landwirtschaft und nötigenfalls abweichend vom Grundsatz der Wirtschaftsfreiheit fördert der Bund die bodenbewirtschaftenden bäuerlichen Betriebe.

Er richtet die Massnahmen so aus, dass die Landwirtschaft ihre multifunktionalen Aufgaben erfüllt. Er hat insbesondere folgende Befugnisse und Aufgaben:

a. Er ergänzt das bäuerliche Einkommen durch Direktzahlungen zur Erzielung eines angemessenen Entgelts für die erbrachten Leistungen, unter der Voraussetzung eines ökologischen Leistungsnachweises.

b. Er fördert mit wirtschaftlich lohnenden Anreizen Produktionsformen, die besonders naturnah, umwelt- und tierfreundlich sind.

c. Er erlässt Vorschriften zur Deklaration von Herkunft, Qualität, Produktionsmethode und Verarbeitungsverfahren für Lebensmittel.

d. Er schützt die Umwelt vor Beeinträchtigungen durch überhöhten Einsatz von Düngstoffen, Chemikalien und anderen Hilfsstoffen.

e. Er kann die landwirtschaftliche Forschung, Beratung und Ausbildung fördern sowie Investitionshilfen leisten.

f. Er kann Vorschriften zur Festigung des bäuerlichen Grundbesitzes erlassen.

Er setzt dafür zweckgebundene Mittel aus dem Bereich der Landwirtschaft und allgemeine Bundesmittel ein. 


\section{Bundesverfassung BV Art. 104a Ernährungssicherheit}

\section{Zur Sicherstellung der Versorgung der Bevölkerung mit Lebensmitteln schafft der Bund Voraussetzungen für:}

a. die Sicherung der Grundlagen für die landwirtschaftliche Produktion, insbesondere des Kulturlandes;

b. eine standortangepasste und ressourceneffiziente Lebensmittelproduktion;

c. eine auf den Markt ausgerichtete Land- und Ernährungswirtschaft;

d. grenzüberschreitende Handelsbeziehungen, die zur nachhaltigen Entwicklung der Land- und Ernährungswirtschaft beitragen;

e. einen ressourcenschonenden Umgang mit Lebensmitteln.

In den folgenden beiden Unterkapiteln wird vertieft auf das Landwirtschaftsgesetz und das Bundesgesetz über das bäuerliche Bodenrecht eingegangen. Diese beiden Gesetze bilden das Rückgrat der Schweizer Agrargesetzgebung.

\subsection{Das Landwirtschaftsgesetz}

Das LwG umfasst neun Titel (siehe Abbildung 12 ): 1. Allgemeine Grundsätze, 2. Rahmenbedingungen für Produktion und Absatz, 3. Direktzahlungen, 4. Soziale Begleitmassnahmen, 5. Strukturverbesserungen, 6. Forschung und Beratung, Förderung der Pflanzen- und Tierzucht sowie genetische Ressourcen, 7. Pflanzenschutzmittel und Produktionsmittel, 8. Rechtsschutz, Verwaltungsmassnahmen und Strafbestimmungen, 9. Schlussbestimmungen. Die Vielfalt dieser Titel zeigt, dass Landwirtschaftspolitik weit mehr umfasst als Direktzahlungen oder Grenzschutzmassnahmen.

Im Folgenden werden die Kapitel 1-7 zusammengefasst. Auf eine vertiefte Beschreibung der Kapitel 8 und 9 wird an dieser Stelle verzichtet. Es gilt aber festzuhalten, dass insbesondere der Abschnitt zu den Strafbestimmungen und dem Rechtsschutz eine wichtige Bedeutung in der Umsetzung der Agrarpolitik hat, weil die Androhung von Strafen einen wichtigen Einfluss auf das Verhalten der Landwirte haben kann. Ausserdem ist es nicht unüblich, dass sich gewisse Interpretationen von Gesetzen erst durch die Rechtsprechung, d.h. Entscheide von Richtern, etablieren. 


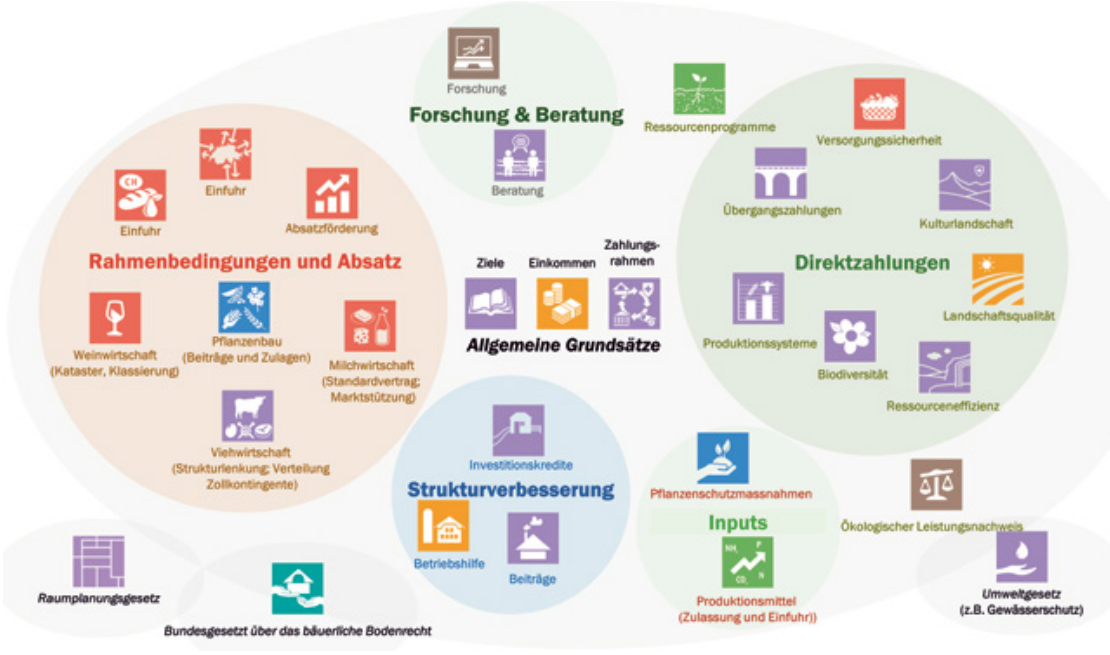

Abbildung 12. Übersicht über die Kernelemente der Gesetzgebung in der Schweizer Agrarpolitik.

In den allgemeinen Grundsätzen des Gesetzes (1. Titel) werden die Ziele der Agrarpolitik aus der Verfassungsgrundlage nochmals aufgenommen und die begrifflichen Grundlagen für die Umsetzung gelegt. Es geht dabei insbesondere um die Definition der Begriffe «landwirtschaftlicher Betrieb», "erschwerte Produktionsbedingungen» oder «landwirtschaftliche Produktion». Zu Letzterem gehören nicht nur die Produktion von Produkten aus dem Pflanzenbau und der Nutztierhaltung, sondern auch die Aufbereitung, Lagerung und Verarbeitung dieser Erzeugnisse (Kernlandwirtschaft). Zudem gehört explizit auch die Bewirtschaftung von naturnahen Flächen zur Kernlandwirtschaft. Landwirtschaftsnahe Tätigkeiten dagegen sind Produkte oder Dienstleistungen, die nicht direkt zur Kernlandwirtschaft gehören, aber eng mit dieser verknüpft sind wie beispielsweise Agrotourismus.

Der Begriff der Kernlandwirtschaft ist deshalb wichtig, weil viele Instrumente der Agrarpolitik nur dann zur Anwendung kommen, wenn der Betrieb eine gewisse Grösse aufweist. Dabei ist es entscheidend, ob beispielsweise die Verarbeitung der eigenen Produkte als landwirtschaftliche Aktivität gilt. Je nachdem kann ein Betrieb in diesem Fall von Privilegien der Landwirtschaft profitieren oder nicht. Mit anderen Worten: Nicht jeder Stall gehört automatisch zu einem landwirtschaftlichen Betrieb und nicht alles, was Landwirtinnen und Landwirte tun, ist automatisch Landwirtschaft. Der Gesetzgeber definiert über die Begriffe «landwirtschaftlicher Betrieb» und «Kernlandwirtschaft», wen und welche Tätigkeiten er als landwirtschaftlich ansieht und damit letztendlich förderungswürdig findet. Was trivial klingen mag, kann in der Praxis zu grossen Herausforderungen führen, wenn beispielsweise neue Produktionsformen (z.B. genossenschaftliche) oder Produkte (z.B. insektenbasierte) entstehen. 
Im Artikel 5 wird auch der Begriff Einkommen definiert. Dieser Artikel sieht vor, dass ökonomisch leistungsfähige Betriebe im Durchschnitt über mehrere Jahre ein Einkommen erzielen, dass mit der übrigen erwerbstätigen Bevölkerung in der Region vergleichbar ist. Diese Formulierung lässt viel Spielraum für Interpretation. Der Bundesrat vergleicht jeweils das dreijährige Mittel des obersten Viertels der Buchhaltungsbetriebe mit dem Einkommen der übrigen Bevölkerung. Bäuerliche Interessenvertreter argumentieren dagegen oft mit dem Durchschnittswert der landwirtschaftlichen Einkommen, die tiefer liegen als der Mittelwert der übrigen Bevölkerung (siehe Abschnitt 5.2). Die politische Diskussion dreht sich auch darum, ob nicht das Haushaltseinkommen ein besserer Indikator für den Vergleich sei oder ob der Durchschnitt aller Erwerbstätigen oder nur derjenige von Selbstständigen als Vergleichswert herbeigezogen werden soll. Unabhängig von dieser Diskussion erlaubt der Artikel 5 dem Bundesrat, Massnahmen zu ergreifen, welche die Einkommenssituation in der Landwirtschaft verbessern.

Schliesslich ist im letzten Artikel der allgemeinen Grundsätze festgehalten, dass das Agrarbudget alle vier Jahre mit einem einfachen Bundesbeschluss, eine einfache Mehrheit genügt, bestätigt werden muss. Das Agrarbudget umfasst jährliche Zahlungen von ungefähr 3,6 Mia. CHF. Der grösste Anteil, rund drei Viertel der Ausgaben, machen dabei die Direktzahlungen aus. Für den Bereich Produktion und Absatz werden ca. $12 \%$, für die Strukturverbesserungen rund $4 \%$ ausgegeben. Die restlichen knapp $8 \%$ werden für andere Dienstleistungen wie Forschung, Beratung und Administration aufgewendet. Zu den Ausgaben des Bundes kommen noch weitere Zahlungen der Kantone sowie deren administrativen Aufwände dazu. Insgesamt belaufen sich die jährlichen Ausgaben für die Landwirtschaft in der Schweiz damit auf etwas mehr als 4 Mia. CHF. Die Höhe dieser Ausgaben blieb in den letzten zwölf Jahren ungefähr konstant. Da gleichzeitig die gesamten Ausgaben des Bundes zunahmen, sank der relative Anteil der Ausgaben für die Landwirtschaft in den letzten Jahren.

\subsubsection{Rahmenbedingungen für Produktion und Absatz}

Der zweite Titel des Landwirtschaftsgesetzes befasst sich mit den Rahmenbedingungen für die Produktion und den Absatz von landwirtschaftlichen Produkten. Aus dem Kapitel 8 ging hervor, dass die Schweizer Landwirtschaft durch bäuerliche, tendenziell kleinstrukturierte Betriebe geprägt ist. Diese weisen im Vergleich zu anderen Ländern hohe Produktionskosten auf. Daneben operiert die Schweizer Landwirtschaft in einem generell hohen Kostenumfeld, in erster Linie mit Bezug zu den Löhnen. Schliesslich führt die X-Struktur der Wertschöpfungskette dazu, dass die Landwirtschaft über wenig Marktmacht verfügt und teilweise mit oligopsonistischen und monopolistischen Strukturen konfrontiert ist. Der Gesetzgeber versucht diesen beiden Herausforderungen, d.h. hohe Produktionskosten und unvollständige Märkte, entgegenzuwirken. Dadurch sollen im Grundsatz eine kostengünstige Produktion gefördert 
und möglichst hohe Markterlöse geschaffen werden. Das Gros der Massnahmen bezieht sich aber auf die Sicherung der Preise für landwirtschaftliche Produkte und damit auf das Ziel, möglichst hohe Markterlöse zu erzielen. Der Bund gibt jährlich knapp 420 Mio. CHF für den Bereich Produktion und Absatz aus.

Der Titel «Rahmenbedingungen für Produktion und Absatz» umfasst fünf Kapitel. Im ersten Kapitel geht es um allgemeine wirtschaftliche Bestimmungen, insbesondere um Qualitätsvorschriften, Kennzeichnung, Absatzförderung, Marktentlastung und Einfuhrregelungen. Zentral für viele Massnahmen im Bereich der Produktion und Absatz ist das Prinzip der Selbsthilfe. Dieses Prinzip sieht vor, dass die Förderung der Qualität und des Absatzes sowie die Abstimmung von Angebot und Nachfrage auf den Produktemärkten in erster Linie Sache der Produzenten- und Branchenorganisationen ist. Diese Organisationen dürfen beispielsweise Richtpreise festlegen und Standardverträge ausarbeiten. Der Bund kann Selbsthilfemassnahmen unterstützen oder bei ausserordentlichen Entwicklungen Kosten von Marktentlastungen übernehmen. Wiederkehrende Marktentlastungen übernimmt der Bund beispielsweise beim Überangebot von Eiern nach Ostern oder bei Schafwolle.

Grundsätzlich greift er aber nicht direkt in die inländischen Agrarmärkte ein. Die Anpassung der Produktion an den Markt ist folglich Sache der Organisationen der Produzentinnen und Produzenten bzw. der entsprechenden Branchen und nicht in erster Linie eine Sache des Bundes. Dies ist ein wichtiger Unterschied zur Agrarpolitik im letzten Jahrhundert, in welchem der Staat Absatzmengen und Preise direkt regelte. Trotzdem blieb aber die Regelungsdichte und die Steuerung von Angebot und Nachfrage auch nach der Agrarreform in den 1990er-Jahren hoch. Die Aufgaben wurden jedoch vom öffentlich-rechtlichen in einen privatrechtlichen Rahmen überführt. Auf den heute bestehenden Märkten sind Branchenorganisationen wie Proviande für Fleisch, BOM für Milch oder swiss granum für Getreide (Box 12) zentrale Akteure in Bezug auf Marktregelungen. Aus einer ordnungspolitischen Sicht ist diese Steuerung des Angebots durch die Branchenorganisationen problematisch. Theoretisch ist damit zu rechnen, dass die Branche dadurch - analog zu einem Kartell - Renten erwirtschaften kann. In der Praxis kommt aber aufgrund der ohnehin unvollkommenen Marktsituation kaum Kritik an dieser Marktordnung auf. Die Branchenorganisationen sind dadurch einflussreiche Akteure im Agrar- und Lebensmittelsektor. 


\section{Box 12 Beispiel für eine Branchenorganisation: swiss granum}

Swiss granum ist die schweizerische Branchenorganisation für Getreide, Ölsaaten und Eiweisspflanzen. Sie wurde 1999 gegründet und hat die rechtliche Form eines Vereins. Der Verein setzt sich aus 17 Mitgliedern der Wertschöpfungsketten für Brotgetreide, Futtergetreide und Eiweisspflanzen sowie Ölsaaten zusammen, d.h., er vereinigt Produzenten, Sammelstellen, Händler, Müller, Futtermittelhersteller, Hersteller von Speiseölen und -fetten, Bäcker, Detailhändler sowie Nutztierhalter unter einem Dach. Swiss granum fördert die Zusammenarbeit entlang der Wertschöpfungsketten und sie koordiniert und vertritt die Interessen der gesamten Branche gegenüber Dritten (z.B. der Administration). Das Ziel des Vereins ist es, verlässliche politische und wirtschaftliche Rahmenbedingungen und konsensfähige Lösungen für aktuelle und zukünftige Herausforderungen der gesamten Branche zu entwickeln.

Die Hauptaufgabengebiete von swiss granum umfassen:

\section{Schaffung von Marktransparenz}

○ Schätzung der Anbauflächen und Produktionsmengen vor und Erhebung der Erntemengen während und nach der Ernte.

○ Festlegung von Richtpreisen und Publikation der durchschnittlichen Marktpreise für Ölsaaten.

○ Erhebung der Erntequalität des Brotweizens (Schnelltest, Laboranalysen, Backtest).

○ Durchführung der Qualitätstagung.

2. Verbesserung/Sicherstellung der Qualität

○ Festlegung von Übernahmebedingungen für Getreide, Ölsaaten und Eiweisspflanzen.

○ Orientierung der Produzenten über die Marktbedürfnisse mit entsprechenden Empfehlungen.

○ Abgabe von Empfehlungen bezüglich Qualitätssicherung und Lebensmittelsicherheit (Mykotoxin-Monitoring).

- Erstellung und Publikation der Listen der empfohlenen Sorten sowie Koordination und Kofinanzierung der dazu nötigen Anbauversuche.

3. Engagement zugunsten der Rahmenbedingungen und Vertretung gemeinsamer Interessen

○ Stellungnahmen, Verhandlungen, Kontakte mit der Verwaltung und den Partnern. 
Interne und externe Kommunikation: Medienarbeit, Webseite, Vorträge etc.

4. Treuhänderisches Inkasso produktgebundener Mitgliederbeiträge für verschiedene Mitgliederorganisationen und Führung des Sekretariats des Vereins Schweizer Brot und des Vereins Schweizer Rapsöl.

Swiss granum finanziert sich zum grössten Teil aus produktgebundenen Mitgliederbeiträgen, welche je hälftig von Produzenten sowie Handel/Verarbeitung erbracht werden. Ausserdem generiert sie Einnahmen durch die Übernahme von Mandaten, das Erbringen von Dienstleistungen sowie durch die Jahresbeiträge der Mitglieder. Quelle: swissgranum.ch

Neben dem Prinzip der Selbsthilfe versucht der Gesetzgeber Grundlagen zu schaffen, dass die Bäuerinnen und Bauern einen möglichst hohen Markterlös erzielen können. Dabei verfolgt die Schweizer Landwirtschaftspolitik eine Qualitätsstrategie. Die grundsätzliche Überlegung ist, dass die Schweizer Landwirtschaft Produkte mit einer hohen Qualität herstellen sollte, um diese auf Absatzmärkten mit einer hohen Zahlungsbereitschaft für ebendiese Qualität zu verkaufen.

Das Ziel ist, dem Preiswettbewerb, der für die Schweiz aufgrund der hohen Produktionskosten wenig Spielraum lässt, durch eine gezielte Differenzierung und Auslobung der Produktion auszuweichen. Ein wichtiges Instrument in diesem Kontext ist die Kennzeichnung. Sie erlaubt eine Differenzierung der Produktion z.B. nach Herkunft (z.B. Berg) oder Produktionsform (z.B. Bio) und schützt die entsprechenden Produkte vor einer preiswerteren Imitation. Dabei spielen insbesondere auch die Ursprungskennzeichnungen wie AOP (Appellation d'Origine Protégée), AOC (Appellation d'Origine Contrôlée) und IGP (Indication Géographique Protégée) eine zentrale Rolle, weil diese auch im Ausland und damit auf den ausländischen Märkten anerkannt werden. Darüber hinaus unterstützt der Bund mit der Absatzförderung die Werbung und Promotion von landwirtschaftlichen Produkten aus der Schweiz im Inund Ausland. Die Werbung darf sich dabei aber nicht auf einzelne Produkte beziehen, sondern muss dem Interesse der gesamten Branche dienen wie z.B. die Werbung für Schweizer Milch mit der Kuh Lovely. 


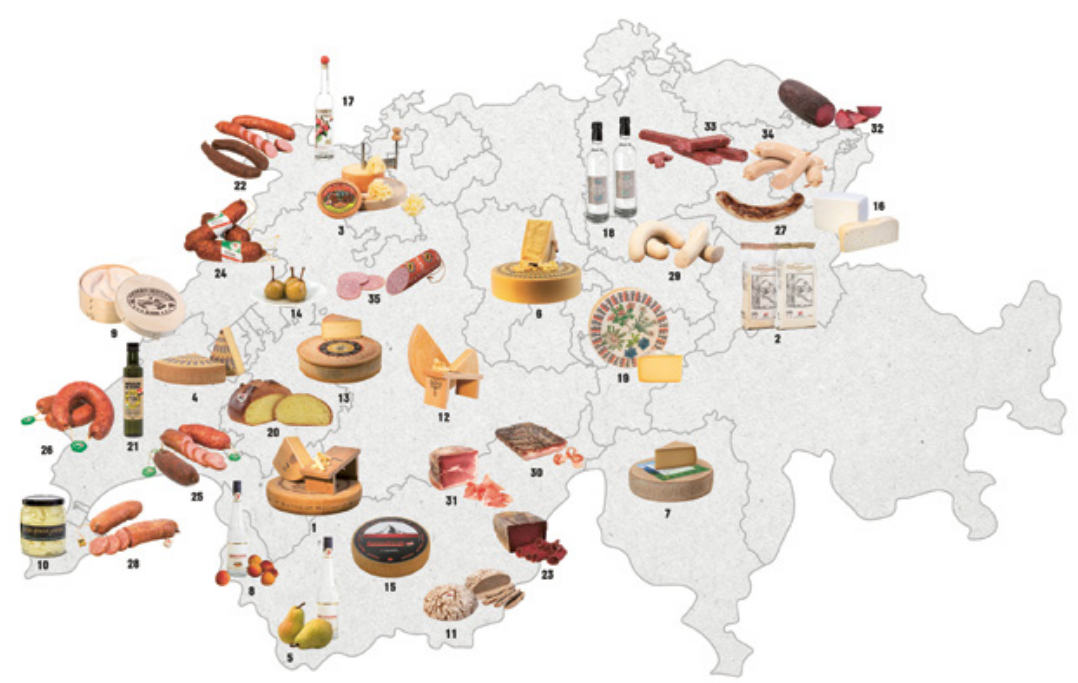

Abbildung 13. Karte der Schweizer Produkte mit einer Ursprungskennzeichnung AOP (121) und IGP (22-35). 1. L'Etivaz, 2. Rheintaler Ribel, 3. Tête de Moine, 4. Gruyère, 5. Eaude-vie de poire du Valais, 6. Sbrinz, 7. Formaggio d'alpe ticinese, 8. Apricotine, 9. Vacherin Mont-d'Or, 10. Cardon épineux genevois, 11. Walliser Roggenbrot, 12. Berner Alpkäse, 13. Vacherin fribourgeois, 14. Poire à Botzi, 15. Walliser Raclette, 16. Werdenberger Sauerkäse, 17. Damassine, 18. Zuger Kirsch, 19. Glarner Alpkäse, 20. Cuchaule, 21. Huile de noix vaudoise, 22. Saucisse d'Ajoie, 23. Walliser Trockenfleisch, 24. Saucisson neuchâtelois, 25. Saucisson vaudois, 26. Saucisse aux choux vaudoise, 27. St. Galler Bratwurst, 28. Longeole, 29. Glarner Kalberwurst, 30. Walliser Trockenspeck, 31. Walliser Rohschinken, 32. Appenzeller Mostbröckli, 33. Appenzeller Pantli, 34. Appenzeller Siedwurst, 35. Berner Zungenwurst. Weitere Produkte: Munder Safran, Emmentaler Schweiz (AOP), Bünderfleisch, Zuger Kirschtorte (IGP).

Ein zentrales Element für die Förderung der Produktion ist die Einfuhrregelung bzw. der Grenzschutz für landwirtschaftliche Produkte. Damit verhindert der Bund den Import von billigeren Nahrungsmitteln aus dem Ausland und ermöglicht damit den einheimischen Produzentinnen und Produzenten höhere Preise. In der Schweiz dienen zur Regelung der Einfuhr Schwellenpreise, z.B. für Saatgetreide, Futtermittel und Ölsaaten, sowie Zollkontingente für die meisten übrigen Produkte. Die allermeisten Produktionsbereiche der Schweizer Landwirtschaft sind durch die Einfuhrregelungen stark geschützt. Eine Ausnahme bildet der Käsefreihandel mit der EU. Käse kann zwischen den europäischen Ländern und der Schweiz zollfrei gehandelt werden. Im Gegensatz zum Käse, der sogenannten «gelben Linie», ist die «weisse Linie», d.h. Trinkmilch, Joghurt etc. geschützt. 
Der Abschnitt zum Grenzschutz nimmt im Landwirtschaftsgesetz nur wenig Platz ein. Seine Wirkung ist aber enorm. Fast die Hälfte der totalen Stützung des Agrarsektors geht auf den Grenzschutz zurück (siehe dazu auch das Kapitel 4.2). Auch in den politischen Prozessen ist die Einfuhrreglementierung ein wichtiger Aspekt. Die Schweiz ist eine kleine offene Volkswirtschaft, die auf bilaterale oder multilaterale Handelsabkommen angewiesen ist. In Verhandlungen über Freihandelsabkommen spielt die Landwirtschaft immer eine wichtige Rolle. Als Kompensation für Zugeständnisse im Industrie- und Dienstleistungsbereich fordern Länder, die Agrarprodukte exportieren, einen besseren Zugang zum Schweizer Agrarmarkt. Entsprechende Konzessionen der Schweiz senken den Grenzschutz und haben damit einen direkten Effekt auf das landwirtschaftliche Preisniveau in der Schweiz (siehe hierzu den Abschnitt 4.1 zur WTO).

Schliesslich enthalten die allgemeinen wirtschaftlichen Bestimmungen auch den Auftrag, Marktbeobachtungen durchzuführen, Einschränkungen zur Produktion von gentechnisch veränderten Lebensmitteln und Regelungen zum Patentschutz auf Produktionsmittel oder landwirtschaftlichen Investitionsgütern. Die Einschränkungen in Bezug auf die Gentechnik gehen auf die Volksinitiative «für Lebensmittel aus gentechnikfreier Landwirtschaft» im Jahr 2005 zurück. Eigentlich als Moratorium für fünf Jahre vorgesehen, wurden die entsprechenden Regeln weitergeführt, d.h. ein Verbot der Einführung und des Inverkehrbringens von gentechnisch veränderten Pflanzen (inkl. Pflanzenteile und Saatgut) und Tieren.

Das zweite Kapitel im Bereich «Rahmenbedingungen für Produktion und Absatz» fokussiert auf die Milchwirtschaft. Ein Kernelement ist auch hier die Festlegung eines Standardvertrags durch die Branchenorganisation Milch. Damit soll eine privatwirtschaftliche Regelung des Milchmarkts erreicht werden, welche den Wettbewerb nicht zu stark einschränkt. Darüber hinaus werden im Milchmarkt drei spezifische Marktstützungen vorgenommen: 1) die Zulage für verkäste Milch; 2) die Zulage für Fütterung ohne Silage; 3) die Zulage für Verkehrsmilch. Die Zulage für verkäste Milch ermöglicht, den Kostennachteil des Rohstoffs Milch in der Käseproduktion gegenüber den Käseherstellern in der EU zu kompensieren. Es handelt sich um eine sogenannte Rohstoffverbilligung. Die Zulage für Fütterung ohne Silage gilt die höheren Produktionskosten der heubasierten Fütterung ab. Silage ist zwar günstiger in der Produktion, bei der Herstellung von Rohmilchkäse kann es jedoch zu einer Fehlgärung durch die vorhandenen Buttersäurebakterien kommen. Um die Rohstoffproduktion für die wertschöpfungsstarken Rohmilchkäse wie Emmentaler, Gruyère oder Sbrinz zu sichern, bezahlt der Bund eine Kompensation.

Die Zulage für Verkehrsmilch schliesslich ist eine Kompensationsmassnahme, welche einer Rohstoffverbilligung für andere Exportprodukte als Käse entspricht. Diese Zulage ersetzte die Massnahmen im Bereich des sogenannten "Schoggigesetzes», das bis Ende 2018 die Milch und das Getreide verbilligte, welche für die Produktion von 
exportierter Schokolade und Biskuits verwendet wurden. Aktuell bezahlt der Bund eine Zulage von 4,5 Rappen pro kg Milch an die Produzentinnen und Produzenten. Die Verarbeiter ziehen diesen Betrag wiederum vom Milchpreis ab. Für Milch, die nicht verkäst wird, bezahlt der Verarbeiter diesen Betrag in zwei Fonds ein. $80 \%$ dieser Gelder gehen in die Rohstoffverbilligung von Exportprodukten wie Schokolade und ersetzen damit die frühere Exportsubvention mit einer privatrechtlichen Regelung zwischen Produzentinnen und Produzenten und den Verarbeitern. In einem zweiten Fonds werden maximal 20 \% dieser Zulage für den Export von Milchfett auf den Weltmarkt eingesetzt. Sofern keine C-Milch produziert wird, kann diese Einzahlung durch die Branchenorganisation Milch sistiert werden.

Das dritte Kapitel umfasst Regelungen zur Viehwirtschaft. Als zentrales Instrument dient hier die Strukturlenkung. Das bedeutet, dass der Bund Vorschriften zu den Höchstbeständen je Betrieb und Tierart erlässt. Darüber hinaus regelt das Kapitel die Verteilung der Zollkontingente und der Qualitätsbeurteilung im Fleischbereich. Der Bund kann auch Massnahmen mitfinanzieren, die den Fleischmarkt entlang der gesamten Wertschöpfungskette entlasten, oder er kann Dritte beauftragen, solche Massnahmen umzusetzen. Schliesslich regelt der Bund in diesem Kapitel den Absatz von Schafwolle und die Entlastung des inländischen Eiermarkts durch Beiträge.

Das vierte Kapitel befasst sich mit dem Pflanzenbau. Das wichtigste Instrument ist der sogenannte Einzelkulturbeitrag. Dieser wird für den Anbau von spezifischen Ackerkulturen ausbezahlt, die für die Versorgungssicherheit wichtig sind. Dazu gehören in erster Linie Raps, Sonnenblumen und Zuckerrüben. In Bezug auf Zucker ist darauf hinzuweisen, dass im Gesetz festgehalten ist, dass mit der Unterstützung von Zuckerrüben auch die Funktionsfähigkeit der Verarbeitungskette aufrechterhalten bleiben soll. Damit ist in erster Linie die Erhaltung der Zuckermühlen in Aarberg und Frauenfeld gemeint. Die Liefermengen werden zwischen den Zuckerfabriken und den Landwirten vertraglich abgesprochen. Neben diesen drei Kulturen wird auch die Produktion von Saatgut für Kartoffeln, Mais, Futtergräsern und Futterleguminosen mit Einzelkulturbeiträgen unterstützt. Analog wie im Zuckeranbau braucht der Bewirtschafter oder die Bewirtschafterin eine Vereinbarung mit einer Saatgutvermehrungsorganisation, in welcher die Anbaufläche vertraglich festgelegt wird. Ausserdem werden Soja, Ackerbohnen, Eiweisserbsen und Lupinen zu Futterzwecken ebenfalls mit Einzelkulturbeiträgen unterstützt. Dadurch soll sichergestellt werden, dass genügend Futter, insbesondere Eiweisskomponenten für die inländische Tierproduktion, zur Verfügung steht. Neben den Einzelkulturbeiträgen sind im Kapitel Pflanzenbau auch Zulagen für den Getreideanbau vorgesehen. Analog zu den Zulagen für Verkehrsmilch wurden diese Subventionen im Januar 2019 als Ersatz für das «Schoggigesetz» eingeführt. Auch in diesem Fall wird die Verwendung der Zulage über die Branchenorganisation gesteuert. Die Zulage erlaubt den Branchenorganisationen, den Rohstoff für die Exportprodukte weiterhin zu verbilligen: Die Landwirte alimentieren einen Fonds, 
der innerhalb der Branche zur Steuerung des Absatzes verwendet wird. Da es sich aber, im Gegensatz zum «Schoggigesetz», um eine privatrechtliche Regelung handelt, fällt sie gemäss WTO-Recht nicht unter die Bezeichnung Exportsubvention.

Das fünfte Kapitel befasst sich mit der Weinwirtschaft. Die Massnahmen in diesem Bereich sehen keine direkten Subventionen vor, sondern regeln in erster Linie die Qualität im Weinbau durch Bewilligungen, Klassierungen und Kontrollen.

\subsubsection{Direktzahlungen}

Der dritte Titel im Landwirtschaftsgesetz befasst sich mit den Direktzahlungen. Diese sind direkt auf die in der Verfassung festgehaltenen Ziele ausgerichtet und umfassen folgende Kategorien: Kulturlandschaftsbeiträge, Versorgungssicherheitsbeiträge, Biodiversitätsbeiträge, Landschaftsqualitätsbeiträge, Produktionssystembeiträge und Ressourceneffizienzbeiträge (Box 13 und Tabelle 1). Diese Kategorisierung wurde im Jahr 2014 im Rahmen der Agrarpolitikreform AP14-17 eingeführt (siehe Geschichte der Schweizer Agrarpolitik im Kapital 7). Das System mit einer klaren Zielausrichtung löste die bis dahin bestehende Ausgestaltung ab, welche neben den allgemeinen Direktzahlungen und den ökologischen Direktzahlungen auch noch Beiträge kannte, die pro Tier ausbezahlt wurden. Letztere haben jedoch einen unerwünschten Produktionseffekt und wurden deshalb abgeschafft. Um den Übergang vom alten zum neuen System möglichst abzufedern und den Landwirten Zeit zu geben, sich anzupassen, wurden zusätzlich noch sogenannte Übergangsbeiträge eingeführt. Diese sind degressiv ausgestaltet, d.h., sie werden über einen Zeitraum von acht Jahren jedes Jahr reduziert, d.h. bis ca. 2023. Insgesamt umfassen die Direktzahlungen vom Staat zu den Landwirten rund 2,8 Mia. CHF.

Das Landwirtschaftsgesetz definiert im ersten Teil des Titels «Direktzahlungen» die Voraussetzungen für den Erhalt dieser Beiträge. Diese lassen sich in zwei Kategorien unterscheiden. Einerseits gibt es soziale Kriterien für den Erhalt von Direktzahlungen. Dazu gehören unter anderem, dass nur bodenbewirtschaftende und bäuerliche Betriebe unterstützt werden. Diese müssen eine Mindestgrösse aufweisen und der Bewirtschafter muss eine landwirtschaftliche Ausbildung absolviert haben. Ausserdem muss mindestens $50 \%$ der anfallenden Arbeit von betriebseigenen Arbeitskräften, d.h. von der Betriebsleiterfamilie oder Angestellten mit Arbeitsvertrag ausgeführt werden. Andererseits müssen die landwirtschaftlichen Betriebe einen ökologischen Leistungsnachweis erfüllen. Dieser legt im Kern fest, dass die Tiere artgerecht gehalten werden und eine ausgeglichene Düngerbilanz, d.h. ein geschlossener Nährstoffkreislauf angestrebt wird. Darüber hinaus braucht es einen angemessenen Anteil an Biodiversitätsförderflächen (7\%) sowie eine geregelte Fruchtfolge und einen geeigneten Bodenschutz. Auch eine gezielte Auswahl und Anwendung von Pflanzenschutzmitteln gehört zum ökologischen Leistungsnachweis. 


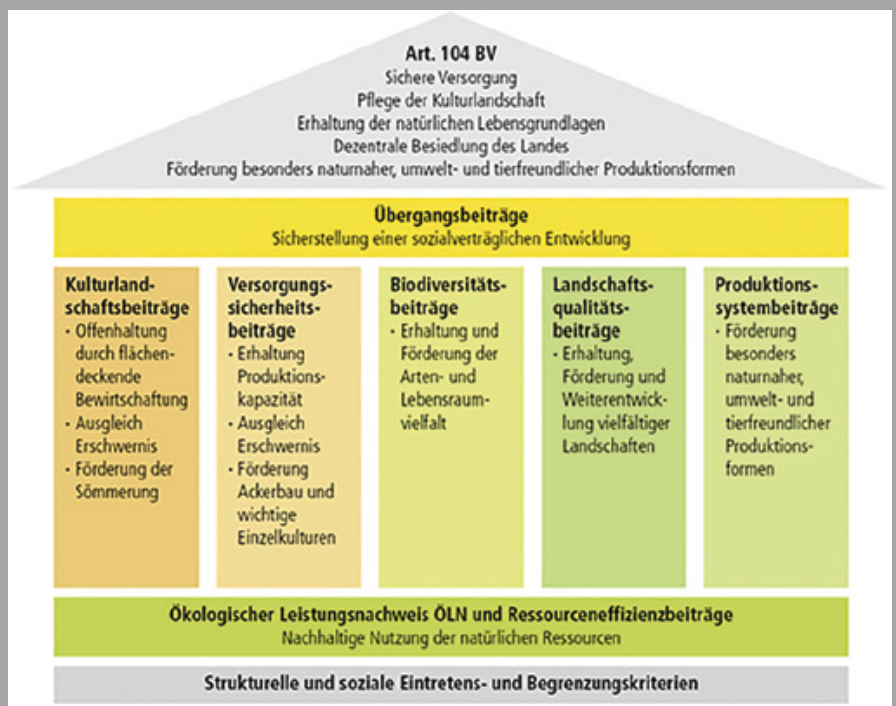

Quelle: Bundesamt für Landwirtschaft

Die Basis für den Erhalt von Direktzahlungen bilden strukturelle und soziale Eintretens- und Begrenzungskriterien (bspw. Mindestgrösse des Betriebes oder landwirtschaftliche Ausbildung). Gleichzeitig müssen auch Mindeststandards in Bezug auf Umweltkriterien eingehalten werden (Gewässerschutz, Tierschutz, Fruchtfolge, Nährstoffbilanz, Anteil ökologischer Ausgleichsflächen etc.). Die Umweltanforderungen sind im sogenannten ökologischen Leistungsnachweis zusammengefasst. Werden diese Kriterien erfüllt, kann der landwirtschaftliche Betrieb Direktzahlungen erhalten. Verletzungen der Umweltkriterien führen zwingend zu einer Kürzung der Zahlungen. Die Zahlungen werden in fünf Kategorien unterteilt, welche jeweils einen Beitrag zu einem spezifischen Ziel der Agrarpolitik leisten sollen. Der Name der Kategorie spricht jeweils direkt das entsprechende Ziel an: Kulturlandschaftsbeiträge, Versorgungssicherheitsbeiträge, Biodiversitätsbeiträge, Landschaftsqualitätsbeiträge und Produktionssystembeiträge. Im Rahmen der Weiterentwicklung der Direktzahlungen in der AP14-17 wurden zusätzlich Übergangsbeiträge eingeführt. Diese sollen eine sozialverträgliche Anpassung der Landwirte an das neue System sicherstellen. Sie sind aber über die Zeit degressiv gestaltet und werden bis 2024 jährlich kleiner. Das konzeptionelle Dach des Direktzahlungssystems wird durch die multifunktionalen Aufgaben der Landwirtschaft aus dem Artikel 104 der Bundesverfassung gebildet. 
Die einzelnen Beiträge wiederum sind nicht nur auf ein bestimmtes Ziel ausgerichtet, sondern ganz spezifisch auf Regionen und Betriebstypen zugeschnitten (siehe dazu den Abschnitt Targeting und Tailoring im Kapitel Instrumente der Agrarpolitik). Die Höhe der Beiträge hängt massgeblich von der Zielausrichtung und der Zielgruppe ab. Insgesamt gibt es mehr als hundert verschiedene Direktzahlungen in Abhängigkeit von Zielen, Produktionszonen (Tal, Hügel, Berggebiete), Landnutzungsformen und Tierarten (Tabelle 1).

Die finanziell bedeutendste Kategorie sind die Versorgungssicherheitsbeiträge. Diese umfassen jährlich rund $1 \mathrm{Mia}$. CHF und sollen die Produktionskapazität und damit die Versorgung der Schweizer Bevölkerung mit Nahrungsmitteln sicherstellen. Dieser Beitrag ist in drei Unterkategorien unterteilt:

1) Ein Basisbeitrag von 900 Franken wird für jede Hektare ausbezahlt, die landwirtschaftlich genutzt wird. Ausgenommen sind diejenigen Flächen, die als Biodiversitätsförderflächen gemeldet sind. Auf diesen Flächen wird nur die Hälfte des Basisbeitrags ausbezahlt. Ausserdem wird der Basisbeitrag ab einer Betriebsgrösse von 60 ha gekürzt. Betriebe, die grösser sind als 140 ha, erhalten keinen Basisbeitrag mehr.

2) Zur Erhaltung und Förderung der Ackerkulturen wird auf der offenen Ackerfläche ein Betrag von 400 Franken pro Hektare ausbezahlt.

3) Schliesslich gehört auch der Produktionserschwernisbeitrag zu den Versorgungssicherheitsbeiträgen. Dieser ist nach Zonenzugehörigkeit abgestuft und liegt bei 240 Franken pro Hektare in der Hügelzone und bei 360 Franken in der Bergzone IV.

Für die Kategorie der Kulturlandschaftsbeiträge werden jährlich rund 500 Mio. CHF aufgewendet. Dazu gehören sechs verschiedene Direktzahlungen. Der sogenannte Offenhaltungsbeitrag soll dazu beitragen, eine offene Kulturlandschaft zu erhalten. Er wird in Abhängigkeit der Zonenzugehörigkeit ausbezahlt und beläuft sich auf 100 Franken in der Hügelzone und 390 Franken in der Bergzone IV. Neben dem Offenhaltungsbeitrag werden auch Beiträge ausbezahlt, welche die landwirtschaftliche Nutzung in Hang- und Steillagen sicherstellen soll. 
Tabelle 1: Direktzahlungen in der Schweizer Landwirtschaft

\begin{tabular}{|c|c|c|c|c|c|c|}
\hline $\begin{array}{l}\text { Ziel } \\
\text { (Targe- } \\
\text { ting) }\end{array}$ & $\begin{array}{l}\text { Direkt- } \\
\text { zahlung }\end{array}$ & $\begin{array}{l}\text { An- } \\
\text { zahl }\end{array}$ & $\begin{array}{l}\text { Zugeschnitten } \\
\text { (Tailored) auf ... }\end{array}$ & Design & $\begin{array}{l}\text { Budget } \\
\text { (Mio. } \\
\text { CHF) }\end{array}$ & $\begin{array}{l}\text { Anteil } \\
(2020)^{* *}\end{array}$ \\
\hline $\begin{array}{l}\text { Ver- } \\
\text { sorgungs- } \\
\text { sicherheit }\end{array}$ & $\begin{array}{l}\text { Ver- } \\
\text { sorgungs- } \\
\text { sicherheits- } \\
\text { beitrag }\end{array}$ & 8 & $\begin{array}{l}\text { Produktionszonen } \\
\text { (abnehmend mit } \\
\text { Höhenstufe); Halbie- } \\
\text { rung der Beiträge für } \\
\text { BFF; Zusatzbeitrag } \\
\text { für Ackerflächen }\end{array}$ & $\begin{array}{l}\text { Inputorientierte } \\
\text { Massnahme } \\
\text { (Zahlung pro ha) }\end{array}$ & 800 & $32 \%$ \\
\hline \multirow{5}{*}{$\begin{array}{l}\text { Pflege } \\
\text { der Kul- } \\
\text { turland- } \\
\text { schaft }\end{array}$} & $\begin{array}{l}\text { Kultur- } \\
\text { landschafts- } \\
\text { beitrag }\end{array}$ & 5 & $\begin{array}{l}\text { Produktionszonen } \\
\text { (zunehmend mit Hö- } \\
\text { henstufe; keine Zah- } \\
\text { lung im Talgebiet) }\end{array}$ & $\begin{array}{l}\text { Inputorientierte } \\
\text { Massnahme } \\
\text { (Zahlung pro ha) }\end{array}$ & 140 & $6 \%$ \\
\hline & $\begin{array}{l}\text { Hang- und } \\
\text { Steillagen- } \\
\text { beiträge }\end{array}$ & 7 & $\begin{array}{l}\text { Steilheit der Parzelle } \\
\text { (spezifische Zahlun- } \\
\text { gen für Reben) }\end{array}$ & $\begin{array}{l}\text { Inputorientierte } \\
\text { Massnahme } \\
\text { (Zahlung pro ha) }\end{array}$ & 150 & $6 \%$ \\
\hline & $\begin{array}{l}\text { Sömmerungs- } \\
\text { beiträge }\end{array}$ & 5 & $\begin{array}{l}\text { Tierart: Rinder, } \\
\text { Schafe, Ziegen; Art } \\
\text { von Weide, Herden- } \\
\text { schutzmassnahmen }\end{array}$ & $\begin{array}{l}\text { Inputorientierte } \\
\text { Massnahme } \\
\text { (Zahlung pro } \\
\text { Normalstoss*) }\end{array}$ & 130 & $5 \%$ \\
\hline & $\begin{array}{l}\text { Alpungs- } \\
\text { beiträge }\end{array}$ & 1 & $\begin{array}{l}\text { Betriebe, welche die } \\
\text { Tiere für die Alp «zur } \\
\text { Verfügung stellen» }\end{array}$ & $\begin{array}{l}\text { Inputorientierte } \\
\text { Massnahme } \\
\text { (Zahlung pro Nor- } \\
\text { malstoss) }\end{array}$ & 110 & $4 \%$ \\
\hline & $\begin{array}{l}\text { Beitrag } \\
\text { für Biodiversi- } \\
\text { tätsförder- } \\
\text { flächen Quali- } \\
\text { tätsstufe I }\end{array}$ & 17 & $\begin{array}{l}\text { Produktionszonen } \\
\text { und Art der Fläche } \\
\text { oder Förderelements } \\
\text { (z.B. extensives Gras- } \\
\text { land, Hecken, Acker- } \\
\text { schonstreifen oder } \\
\text { Hochstammobst- } \\
\text { bäume etc.) }\end{array}$ & $\begin{array}{l}\text { Inputorientierte } \\
\text { Massnahme } \\
\text { (Zahlung pro ha; } \\
\text { Elemente werden } \\
\text { in ha Grundfläche } \\
\text { umgerechnet) }\end{array}$ & 160 & $7 \%$ \\
\hline \multirow[t]{2}{*}{$\begin{array}{l}\text { Erhaltung } \\
\text { der Bio- } \\
\text { diversität }\end{array}$} & $\begin{array}{l}\text { Beitrag } \\
\text { für Biodiversi- } \\
\text { tätsförder- } \\
\text { flächen Quali- } \\
\text { tätsstufe II }\end{array}$ & 17 & $\begin{array}{l}\text { Produktionszonen } \\
\text { und Art der Fläche } \\
\text { oder Förderele- } \\
\text { ments. Keine } \\
\text { Zahlung für Mass- } \\
\text { nahmen auf Acker- } \\
\text { flächen }\end{array}$ & $\begin{array}{l}\text { Outcome- } \\
\text { orientierte Mass- } \\
\text { nahme (Zahlung } \\
\text { nur bei Vorhan- } \\
\text { densein von defi- } \\
\text { nierten seltenen } \\
\text { Arten) }\end{array}$ & 160 & $7 \%$ \\
\hline & $\begin{array}{l}\text { Vernetzungs- } \\
\text { beitrag }\end{array}$ & 6 & $\begin{array}{l}\text { Produktionszonen } \\
\text { und Art der Fläche } \\
\text { oder Förderelements }\end{array}$ & $\begin{array}{l}\text { Projektbezogene } \\
\text { Massnahme } \\
\text { (Zahlung pro ha) }\end{array}$ & 105 & $4 \%$ \\
\hline
\end{tabular}




\begin{tabular}{|c|c|c|c|c|c|c|}
\hline $\begin{array}{l}\text { Land- } \\
\text { schafts- } \\
\text { qualität }\end{array}$ & $\begin{array}{l}\text { Landschafts- } \\
\text { qualitätsbei- } \\
\text { träge (LQB) }\end{array}$ & 4 & $\begin{array}{l}\text { Ziele des Projekts } \\
\text { (z.B. ökologische } \\
\text { Elemente oder in } \\
\text { einer Region typi- } \\
\text { sche Landnutzungen } \\
\text { oder Produktions- } \\
\text { formen) }\end{array}$ & $\begin{array}{l}\text { Projektbezogene } \\
\text { Massnahme } \\
\text { (Zahlung pro ha } \\
\text { oder Grossvieh- } \\
\text { einheit auf Söm- } \\
\text { merungsflächen) }\end{array}$ & 150 & $6 \%$ \\
\hline \multirow{5}{*}{$\begin{array}{l}\text { Nachhal- } \\
\text { tige Pro- } \\
\text { duktions- } \\
\text { systeme }\end{array}$} & $\begin{array}{l}\text { Beitrag für } \\
\text { biologische } \\
\text { Landwirtschaft }\end{array}$ & 3 & $\begin{array}{l}\text { Abhängig von } \\
\text { Kulturen und Land- } \\
\text { nutzung (Spezial- } \\
\text { kulturen, Ackerbau } \\
\text { und Grasland) }\end{array}$ & $\begin{array}{l}\text { Inputorientierte } \\
\text { Massnahme } \\
\text { (Zahlung pro ha) }\end{array}$ & 60 & $3 \%$ \\
\hline & $\begin{array}{l}\text { Extenso- } \\
\text { Beitrag }\end{array}$ & 1 & $\begin{array}{l}\text { Zahlung für die } \\
\text { Produktion von } \\
\text { Weizen ohne } \\
\text { Herbizide }\end{array}$ & $\begin{array}{l}\text { Inputorientierte } \\
\text { Massnahme } \\
\text { (Zahlung pro ha) }\end{array}$ & 35 & $1 \%$ \\
\hline & $\begin{array}{l}\text { Grasland- } \\
\text { basierte } \\
\text { Milch- und } \\
\text { Fleischproduk- } \\
\text { tion (GMF) }\end{array}$ & 1 & $\begin{array}{l}\text { Einschränkung } \\
\text { des Einsatzes von } \\
\text { Kraftfutter und Mais } \\
\text { in der Fütterung } \\
\text { (abhängig von Pro- } \\
\text { duktionszonen) }\end{array}$ & $\begin{array}{l}\text { Inputorientierte } \\
\text { Massnahme } \\
\text { (Zahlung pro ha) }\end{array}$ & 110 & $4 \%$ \\
\hline & $\begin{array}{l}\text { Besonders } \\
\text { tierfreundliche } \\
\text { Stallhaltung } \\
\text { (BTS) }\end{array}$ & 3 & Tierart & $\begin{array}{l}\text { Inputorientierte } \\
\text { Massnahme } \\
\text { (Zahlung pro } \\
\text { Grossvieheinheit) }\end{array}$ & 200 & $8 \%$ \\
\hline & $\begin{array}{l}\text { Regelmässiger } \\
\text { Auslauf im } \\
\text { Freien (RAUS) }\end{array}$ & 7 & Tierart & $\begin{array}{l}\text { Inputorientierte } \\
\text { Massnahme } \\
\text { (Zahlung pro } \\
\text { Grossvieheinheit) }\end{array}$ & 85 & $3 \%$ \\
\hline $\begin{array}{l}\text { Res- } \\
\text { sourcen- } \\
\text { effizienz }\end{array}$ & $\begin{array}{l}\text { Ressourcen- } \\
\text { effizienz- } \\
\text { beiträge }\end{array}$ & 19 & $\begin{array}{l}\text { Landwirtschaftliche } \\
\text { Praktiken (Direkt- } \\
\text { saat, Präzisionsland- } \\
\text { wirtschaft, N-redu- } \\
\text { zierte Fütterung bei } \\
\text { Schweinen etc.) }\end{array}$ & $\begin{array}{l}\text { Inputorientierte } \\
\text { Massnahme } \\
\text { (Zahlung pro ha } \\
\text { oder Grossvieh- } \\
\text { einheit) }\end{array}$ & 110 & $4 \%$ \\
\hline $\begin{array}{l}\text { Total } \\
\text { (ohne } \\
\text { Über- } \\
\text { gangsbei- } \\
\text { träge) }\end{array}$ & & 104 & & & 2495 & $100 \%$ \\
\hline
\end{tabular}

* Der Normalstoss entspricht der Haltung einer Grossvieheinheit für die Dauer von 100 Tagen im Sömmerungsgebiet. **\% gerundet. 
Der Hangbeitrag beläuft sich auf 410 Franken bei einer Neigung zwischen 18 und $35 \%$. Bei einer Hangneigung zwischen 35 und $50 \%$ wird ein Beitrag von 700 Franken bezahlt. Die Bewirtschaftung von Flächen, die mehr als 50 \% Neigung aufweisen, wird mit 1000 Franken entschädigt. Der Steillagenbeitrag nimmt in Abhängigkeit des Anteils der Flächen mit einer Neigung von 35 \% linear zu. Wenn eine Fläche einen Anteil von $30 \%$ aufweist, welche eine stärkere Neigung als $35 \%$ hat, dann liegt der Steillagenbeitrag bei 100 Franken pro Hektare. Ist die gesamte Fläche, d.h. $100 \%$ steiler als $35 \%$, dann beträgt der Beitrag 1000 Franken. Einen spezifischen Hangbeitrag gibt es für Rebflächen. Je nach Neigung und Terrassierung liegt dieser zwischen 1500 und 5000 Franken. Schliesslich umfassen die Kulturlandschaftsbeiträge auch zwei Zahlungen, die auf die Erhaltung der Sömmerungsflächen abzielen. Einerseits erhalten Betriebe, die ihre Tiere auf die Alp senden, einen Alpungsbeitrag von 370 Franken pro Normalstoss und Jahr; das entspricht einer raufutterverzehrenden Grossvieheinheit während 100 Tagen. Andererseits erhalten die Sömmerungsbetriebe einen Sömmerungsbeitrag von 400 Franken pro Normalstoss. Für Milchkühe, Milchschafe und Milchziegen wird zusätzlich ein Betrag von 40 Franken ausbezahlt. Werden Schafe ohne Behirtung oder Herdenschutzmassnahmen gehalten, wird der Sömmerungsbeitrag gekürzt.

Die drittgrösste Kategorie der Direktzahlungen sind die Produktionssystembeiträge. Dazu gehören der Beitrag für die biologische Landwirtschaft, ein Beitrag für die extensive Produktion von Getreide, Sonnenblumen, Eiweisserbsen, Ackerbohnen, Lupinen und Raps, der Beitrag für graslandbasierte Milch- und Fleischproduktion (GMF) sowie die Tierwohlbeiträge für besonders tierfreundliche Stallhaltungssysteme (BTS) und regelmässiger Auslauf im Freien (RAUS). Für die biologische Landwirtschaft wird ein zusätzlicher Flächenbeitrag ausbezahlt in der Höhe von 1600 Franken für Spezialkulturen, 1200 Franken für die übrige offene Ackerfläche und 200 Franken für die übrige landwirtschaftliche Nutzfläche. Die extensive Produktion von spezifischen Ackerkulturen wie beispielsweise Getreide beträgt 400 Franken pro Hektare und Jahr. Auch die graslandbasierte Milch- und Fleischproduktion wird über einen Flächenbeitrag von 200 Franken gefördert. Die Landwirte müssen für den Erhalt von GMF-Beiträgen nachweisen, dass 75 \% ihrer gefütterten Trockensubstanz aus Grundfutter besteht, d.h. mehrheitlich aus Gras. Im Berggebiet liegt der Mindestanteil an Grundfutter bei $80 \%$. Die Tierwohlbeiträge schliesslich werden in Abhängigkeit der Tierart in Franken pro Grossvieheinheit bezahlt. Der Betrag beläuft sich generell auf 90 (BTS) bzw. 190 Franken (RAUS). Es gibt aber spezifische Ausnahmen für Zuchtsauen, Mast- oder Wildtiere, welche an den spezifischen Herausforderungen in der entsprechenden Tierhaltung angepasst sind. 
Die Biodiversitätsbeiträge umfassen rund 400 Mio. CHF. Diese lassen sich in zwei Kategorien unterscheiden: Qualitätsbeiträge und Vernetzungsbeiträge. Die Qualitätsbeiträge sind nach Art, Qualität und Zonen abgestuft. Die unterschiedlichen Arten umfassen Förderflächen im Ackerbau wie Buntbrachen, Rotationsbrachen oder Ackerschonstreifen. Im Grasland umfassen Biodiversitätsförderflächen extensive und wenig intensiv genutzte Wiesen, extensive Wieder oder Waldweiden, aber auch andere naturnahe Elemente wie Streuflächen, Hecken oder Hochstammobstbäume. Daneben werden noch artenreiche Nutzungen im Rebbau und auf Sömmerungsflächen zu den Biodiversitätsförderflächen gezählt. Für die Biodiversitätsförderflächen im Grasland wird zwischen zwei Qualitätsstufen unterschieden. Flächen mit einer gewissen Anzahl an seltenen Arten erhalten neben einem Basisbeitrag noch eine zusätzliche Direktzahlung (Beitrag für Qualitätsstufe II). Diese sind doppelt so hoch wie der Beitrag für die Qualitätsstufe I. Für Biodiversitätsförderflächen im Ackerbau gibt es nur Beiträge für die Qualitätsstufe I. Im Rebbau und im Sömmerungsgebiet hingegen existieren nur Beiträge, wenn die Qualitätsstufe II erreicht wird.

Wichtig zu erwähnen ist, dass im Gegensatz beispielsweise zum Produktionserschwernisbeitrag oder den Hangbeiträgen, bei welchen der Beitrag mit zunehmender Höhe zunimmt, die Beiträge für Biodiversitätsförderflächen mit zunehmender Höhenstufe abnehmen. Im Talgebiet liegt beispielsweise der Beitrag für extensive Wiesen bei 1080 Franken (1920 Fr. für die Qualitätsstufe II) pro Hektare. In der Bergzone IV noch bei 440 Franken (1100 Fr. für die Qualitätsstufe II). Das hat mit den natürlichen Produktionsbedingungen und den daraus abgeleiteten Opportunitätskosten einer alternativen Nutzung zu tun. Im Berggebiet ist der finanzielle Verlust, den ein Landwirt durch eine extensive Nutzung erfährt, wesentlich kleiner als im Talgebiet. Im Talgebiet kann auf einer intensiven Wiese ein Ertrag von bis zu 140 Dezitonnen (dt) pro Hektare und Jahr erzielt werden. Im Berggebiet liegt dieser potenzielle Ertrag viel tiefer. Entsprechend weniger Verlust erzielt der Betrieb, wenn er nur den Ertrag einer extensiven Wiese von ungefähr $30 \mathrm{dt}$ erzielt (siehe dazu die theoretischen Ausführungen zum Tailoring im Kapitel 5.4).

Neben den Qualitätsbeiträgen zahlt der Bund Beiträge für die Vernetzung, d.h. die räumliche Koordination, von Biodiversitätsförderflächen. Dabei handelt es sich um eine projektbezogene Direktzahlung. Kantone oder regionale Trägerschaft arbeitet einen gemeinsamen Vernetzungsplan aus, welcher vom Bund genehmigt werden muss. Die Bäuerinnen und Bauern verpflichten sich im Rahmen des Vernetzungsprojekts zu einer extensiven Nutzung der räumlich festgelegten Bewirtschaftungseinheiten und erhalten dafür zusätzlich 1000 Franken pro Hektare und Jahr. Das Vernetzungsprojekt wird vom Bund und Kanton gemeinsam finanziert. Der Bund steuert $90 \%$ und der Kanton jeweils 10 \% zur Finanzierung der Vernetzung bei (Kofinanzierung). 
Die Landschaftsqualitätsbeiträge (LQB) umfassen ungefähr 150 Mio. CHF oder knapp $6 \%$ an den totalen Direktzahlungen. Diese Beiträge sollen die vielfältigen Kulturlandschaften in der Schweiz erhalten, fördern und weiterentwickeln. Landschaftsqualitätsbeiträge gehören, wie die Vernetzungsbeiträge, zur Kategorie der projektbezogenen Direktzahlungen. Die Höhe des Beitrags beläuft sich maximal auf 360 Franken pro Hektare oder 240 Franken pro Normalstoss. Auch im Falle der LQB müssen die Kantone oder eine regionale Trägerschaft Ziele für die Landschaftsentwicklung gemeinsam festlegen und vom Bund bewilligen lassen. Innerhalb jedes Projekts werden mit den Landwirten landschaftsspezifische Bewirtschaftungsvereinbarungen getroffen. Diese umfassen beispielsweise farbige Ackerkulturen, Landschaftselemente wie Bäume oder Biodiversitätsförderflächen oder, in Einzelfällen, regionale Eigenheiten wie Lattenzäune oder Ähnliches. Letztere umfassen etwas mehr als 6 Mio. CHF. Der Bund stellt für die Finanzierung $90 \%$ der benötigten finanziellen Mittel zur Verfügung.

Schliesslich fördert der Bund mit den Ressourceneffizienzbeiträgen eine nachhaltige Nutzung von Boden, Wasser und Luft. Diese Beiträge unterstützen den Einsatz von ressourcenschonenden Techniken, die wirksam die Umweltbelastung der Landwirtschaft reduzieren. Diese sind allerdings zeitlich befristet. Zu den ressourcenschonenden Technologien gehören beispielsweise die Anwendung präziser Applikationstechnik für das Ausbringen von Pflanzenschutzmitteln, die Anschaffung von Reinigungssystemen für Pflanzenschutzmittelspritzen, d.h. die Ausrüstung von Spritzen mit einem Spülsystem, oder die schonende Bodenbearbeitung durch Direkt- oder Mulchsaat. Schliesslich gehört auch die Anwendung von emissionsmindernden Gülleausbringverfahren, z.B. durch den Einsatz von Schleppschläuchen, und damit die Reduktion der Ammoniakemissionen zu den ressourcenschonenden Techniken. Diese drei Techniken erhielten allerdings nur bis 2019 Ressourceneffizienzbeiträge. Bis ins Jahr 2021 wurden eine stickstoffreduzierte Phasenfütterung von Schweinen, die Reduktion von Pflanzenschutzmitteln in Obstanlagen, im Rebbau und im Anbau von Zuckerrüben weiterhin unterstützt. Insgesamt werden rund 60 Mio. CHF für die Ressourceneffizienzbeiträge aufgewendet.

\subsubsection{Nachhaltige Nutzung natürlicher Ressourcen}

Wie die Ressourceneffizienzbeiträgen sind auch die Beiträge im Rahmen von Ressourcenprojekten befristet. Die gesetzliche Grundlage dafür ist im Titel 3a des Landwirtschaftsgesetzes festgehalten. Ähnlich wie bei den projektorientierten Zahlungen im Rahmen der LQB und Vernetzungsbeiträge prüft der Bund regionale oder branchenspezifische Projekte, die zum Ziel haben, die Nutzung natürlicher Ressourcen nachhaltiger zu gestalten. Dazu gehören beispielsweise Projekte mit dem Ziel, Treibhausgasemissionen, Stickstoffemissionen oder den Einsatz von Pflanzenschutzmitteln zu reduzieren. Die Höhe der Beiträge variiert je nach Projekt. Grundsätzlich sollten sie aber die Steigerung der Effizienz mit dem Einsatz von Hilfsstoffen, d.h. Dünger, 
Pflanzenschutzmittel oder von Energie initiieren. Diese Projekte haben den Charakter von Innovationsprogrammen. Sie sollen Erkenntnisse über die Effektivität der subventionierten Neuerungen (z.B. Technologien, Verfahren oder Organisationen) geben. Ausserdem soll getestet werden, ob die Neuerungen auch über die Projektregion respektive -branche hinaus eingesetzt werden kann.

\subsubsection{Soziale Begleitmassnahmen}

Unter dem Titel "Soziale Begleitmassnahmen» werden zwei agrarpolitische Instrumente zusammengefasst. Mit der Betriebshilfe werden bäuerliche Betriebe mit einem zinslosen Darlehen unterstützt, die in eine unverschuldete oder durch veränderte wirtschaftliche Rahmenbedingungen verursachte, finanzielle Bedrängnis geraten sind. Mit dem Darlehen kann beispielsweise die Zinslast der Schulden reduziert werden oder eine ausserordentliche finanzielle Belastung überbrückt werden. Es ist wichtig, dass der Betrieb das Darlehen innerhalb einer festgelegten Zeit (maximal 20 Jahre) zurückzahlen muss. Die Kantone, welche die Darlehen vergeben, achten daher darauf, dass nur wirtschaftlich nachhaltige Betriebe eine Betriebshilfe erhalten. Das zweite Instrument ist die Umschulungsbeihilfe. Damit wird den Bäuerinnen und Bauern ermöglicht, eine Umschulung in einen nicht landwirtschaftlichen Beruf zu finanzieren. Dieses Instrument wurde aber nur selten genutzt und Ende 2019 aufgehoben.

\subsubsection{Strukturverbesserungen}

Die Massnahmen im Bereich der Strukturverbesserungen verfolgen fünf Ziele. Erstens sollen durch die Verbesserung der Betriebsgrundlagen die Produktionskosten von landwirtschaftlichen Betrieben gesenkt werden. Zweitens sollen Strukturverbesserungsmassnahmen die Lebens- und Wirtschaftsverhältnisse im ländlichen Raum, insbesondere im Berggebiet, verbessern. Drittens sollen Kulturland sowie landwirtschaftliche Gebäude vor Verwüstung oder Zerstörung durch Naturereignisse geschützt werden. Viertens können Strukturverbesserungsmassnahmen dazu genutzt werden, um ökologische, tierschützerische und raumplanerische Ziele zu erreichen. Fünftens kann mit diesen Massnahmen der Rückbau von Kleingewässern gefördert werden. Es ist wichtig festzuhalten, dass der Fokus bei den Strukturverbesserungen nicht alleine auf der Landwirtschaft liegt, sondern auf dem gesamten ländlichen Raum und damit auch nachgelagerte Unternehmen unter ganz bestimmten Voraussetzungen von den Investitionshilfen profitieren können.

Zur Erreichung dieser Ziele werden grundsätzlich zwei Formen von Investitionshilfen eingesetzt, die dafür sorgen sollen, dass sich die Landwirtschaft hin zu wettbewerbsfähigeren Strukturen entwickelt, ohne dass sie sich dafür untragbar verschulden muss: einerseits die sogenannten Beiträge und andererseits die Investitionskredite. Der Unterschied zwischen diesen beiden Instrumenten ist, dass Beiträge nicht zu- 
rückbezahlt werden müssen (à fonds perdu) und eine Beteiligung der Kantone voraussetzen, während Investitionskredite als rückzahlbare, zinslose Darlehen definiert sind. Neben der Unterteilung in Beiträge und Investitionskredite wird ausserdem zwischen einzelbetrieblichen und gemeinschaftlichen Massnahmen unterschieden.

Wichtige Voraussetzungen für den Erhalt von Investitionshilfen für einzelbetriebliche Massnahmen sind, dass der Betrieb eine längerfristige Existenz sichert und die Tragbarkeit der Investition gewährleistet ist. Die Betriebe müssen üblicherweise mit ihrem Kreditgesuch ein Betriebskonzept liefern, welches darlegt, wie der Betrieb mittel- und langfristig auf die Marktsituation ausgerichtet ist. Damit wird sichergestellt, dass nur die wirtschaftlich nachhaltigen landwirtschaftlichen Betriebe unterstützt werden. Wichtig ist dabei, dass Strukturverbesserungsmassnahmen ausschliesslich für landwirtschaftliche Gewerbe vergeben werden. Implizit fördert die Schweizer Agrarpolitik damit den Strukturwandel, weil kleinere Betriebe keine finanzielle Unterstützung für ihre Investitionen durch den Bund erhalten.

Einzelbetriebliche Investitionskredite werden als Starthilfe, für den Neu- und Umbau von Wohn- und Ökonomiegebäuden, für Massnahmen zur Diversifizierung im landwirtschaftlichen und landwirtschaftsnahen Bereich und für die Verbesserung der Produktion von Spezialkulturen gewährt. Die Starthilfe ermöglicht es einem Betriebsleiter, wenn er das landwirtschaftliche Gewerbe vor seinem 35 Altersjahr von seinen Eltern übernimmt, ein zinsloses Darlehen aufzunehmen. Dieses ist nach Betriebsgrössen abgestuft und senkt die Verschuldung der jungen Bäuerinnen und Bauern bei einer Betriebsübernahme. Die einzelbetrieblichen Investitionshilfen in neue Ökonomiegebäude umfassen den grössten Budgetposten innerhalb der Strukturverbesserungsmassnahmen. Sie ermöglichen ebenfalls eine Senkung der Schuldlast, wenn die landwirtschaftlichen Betriebe investieren. Einzelbetriebliche Beiträge für Ökonomiegebäude werden nur im Hügel- und Berggebiet bewilligt. Massnahmen im Bereich der Diversifizierung sollen dafür sorgen, dass sich den Betrieben zusätzliche Einkommensmöglichkeiten eröffnen wie beispielsweise im Bereich des Agrotourismus.

Im Fall der gemeinschaftlichen Massnahmen wie beispielsweise Meliorationen, d.h. eine Verbesserung der rationellen Bewirtschaftung durch einen Abtausch von Grundeigentum oder die Bodenverbesserung durch Drainagen, ist es notwendig, dass sie sich auf ein wirtschaftlich abgegrenztes Gebiet beschränken und gleichzeitig der ökologische Ausgleich gefördert wird. Für Massnahmen, die über die Landwirtschaft hinausgehen und nachgelagerte Unternehmen wie Mühlen, Schlachthöfe oder Vermarktungsorganisationen mit einbeziehen, ist es eine zentrale Voraussetzung, dass diese wettbewerbsneutral ausgestaltet sind. Das heisst, durch die Investitionshilfe darf kein bestehendes Gewerbe konkurriert werden. Das typische Beispiel für gemeinschaftliche Investitionshilfen in ländliche Räume sind die sogenannten Projekte re- 
gionaler Entwicklung (PRE). Mit diesen Projekten soll die Produktion von einheimischen und regionalen Produkten unterstützt werden und dadurch die Wertschöpfung in den ländlichen Gebieten erhöht werden.

Insgesamt setzt der Bund jährlich ca. $\mathbf{3 6 0}$ Mio. CHF für die Strukturverbesserungsmassnahmen ein. Die Beiträge machen mit insgesamt 80 Mio. CHF den kleineren Anteil aus. Knapp die Hälfte von allen Strukturverbesserungsmassnahmen entfällt auf den Neu- und Umbau von Wohn- und Ökonomiegebäuden (ca. 170 Mio. CHF). Die zweithöchsten Ausgaben werden für die Starthilfe getätigt, welche sich auf ungefähr 70 Mio. CHF belaufen. Der Anteil für gemeinschaftliche Massnahmen im Boden und Wasserbereich beläuft sich auf ungefähr 60 Mio. CHF pro Jahr; diejenigen für gemeinsame Maschinen oder Genossenschaften auf etwas mehr als 20 Mio. CHF. Für PRE und betriebliche Diversifizierungsmassnahmen entfallen ebenfalls ca. 20 Mio. CHF.

Die Prüfung, Abwicklung und die Kontrolle der Umsetzung der Strukturverbesserungsmassnahmen obliegt den Kantonen, welche die Gesuche jedoch vom Bundesamt für Landwirtschaft genehmigen lassen müssen. Auch die Finanzierung der Massnahmen über einen "Fonds de roulement» wird von den Kantonen geführt. Dieser Fonds hat ein Umlaufvermögen von etwas mehr als 2,5 Mia. CHF. Die effektiven Ausgaben des Bundes sind daher tiefer als die ausbezahlten Strukturverbesserungsmassnahmen, weil der Bund nur die Differenz in den Zinsen begleichen muss. Jährlich entstehen dem Bund dadurch Ausgaben in der Höhe von ca. 130 Mio. CHF. Die Landwirte bezahlen die Investitionskredite im Durchschnitt innerhalb von zwölf Jahren zurück.

\subsubsection{Forschung und Beratung, Förderung der Pflanzen- und Tierzucht sowie genetische Ressourcen}

In den Bereichen "Absatz und Produktion», «Direktzahlungen» sowie "Soziale Begleitmassnahmen» und "Strukturverbesserungen» ging es immer um Instrumente, die direkt die Landwirte adressierten. Das heisst, die Bäuerinnen und Bauern waren die Politikadressaten bzw. die Akteure, deren Verhalten mit den entsprechenden Instrumenten geändert werden sollte. Im sechsten und siebten Titel des Landwirtschaftsgesetzes hingegen geht es um Dienstleistungen, welche der Bund als Unterstützung für die Landwirtschaft zur Verfügung stellt. Dadurch werden in diesen Titeln andere Akteure wie Forschung, Beratung und Administration anvisiert. Insgesamt gibt der Bund pro Jahr rund 200 Mio. CHF für diese Dienstleistungen aus.

Im sechsten Titel des Landwirtschaftsgesetzes wird festgelegt, dass der Bund eigene Forschungsanstalten unterhält, welche wissenschaftliche Grundlagen und Erkenntnisse sowie die technischen Grundlagen für die landwirtschaftliche Praxis, Bildung, Beratung entwickeln. Darüber hinaus soll die Forschungsanstalt auch Grundlagen für agrarpolitische Entscheide liefern und agrarpolitische Massnahmen evaluieren. 
Durch das generierte Wissen soll die Landwirtschaft in ihrem Bestreben unterstützt werden, rationell und nachhaltig zu produzieren.

Das Bundesamt für Landwirtschaft setzt diesen gesetzlichen Auftrag um, indem es die Agroscope, das Kompetenzzentrum des Bundes für landwirtschaftliche Forschung, unterhält und leitet. Seit 2017 besteht Agroscope aus drei Kompetenzbereichen für Forschungstechnologie und Wissensaustausch (Tiere und tierische Produkte, Pflanzen und pflanzliche Produkte, Methodenentwicklung und Analytik) sowie sieben strategischen Forschungsbereichen. Letztere setzen sich aus folgenden Bereichen zusammen: Pflanzenzüchtung, Produktionssysteme Pflanzen, Pflanzenschutz, Produktionssystem Tiere und Tiergesundheit, Mikrobielle Systeme von Lebensmitteln, Agrarökologie und Umwelt sowie dem Bereich Wettbewerbsfähigkeit und Systembewertung. Die Agroscope verfügt über 700 Vollzeitstellen und kostet den Bund ungefähr 190 Mio. CHF pro Jahr. 2020 beschloss der Bundesrat, die zehn Standorte der Agroscope, die über die ganze Schweiz verteilt sind, neu zu organisieren. Agroscope wird künftig aus einem zentralen Hauptstandort in Posieux und je einem regionalen Forschungszentrum in Changins und Reckenholz bestehen. Dadurch sollen bis 2028 die Betriebskosten sinken, ohne die wissenschaftliche Leistungsfähigkeit zu schmälern.

Neben der Agroscope unterstützt das BLW die Generierung von neuem Wissen in der Land- und Ernährungswirtschaft mit der Finanzierung von Forschungsinstitutionen, vor allem dem Forschungsinstitut für biologischen Landbau (FiBL), und von Forschungsprojekten mit ungefähr $9 \mathrm{Mio}$. CHF pro Jahr. Ausserdem unterstützt der Bund auch die «Food and Agricultural Organization oft he United Nations» (FAO) mit einem Betrag von knapp 8 Mio. CHF.

Darüber hinaus legt das Landwirtschaftsgesetz auch fest, dass der Bund die Weitergabe von landwirtschaftlichem Wissen und Know-how durch das landwirtschaftliche Beratungswesen fördern soll. Insgesamt wird dafür ein Betrag von 11 Mio. CHF eingesetzt. Der grösste Anteil davon fliesst in die Agridea (8 Mio. CHF). Diese arbeitet spezialisiertes Fachwissen auf und integriert Erfahrungen und Bedürfnisse aus der Praxis. Dazu gehört beispielsweise die Entwicklung neuer Methoden für die Beratung und Weiterbildung oder die Aus- und Weiterbildung der Beratungsfachleute. Daneben publiziert die Agridea praxistaugliche Beratungsunterlagen und Software. Wichtig ist in diesem Zusammenhang anzumerken, dass die berufliche Ausbildung eine kantonale Angelegenheit ist.

Zusätzlich zur Forschung und Beratung fördert die Schweizer Agrarpolitik auch die Pflanzen- und Tierzucht sowie die Erhaltung von genetischen Ressourcen. Der Bund soll die Züchtung von Nutzpflanzen fördern, die ökologisch und qualitativ hochwertig und den hiesigen Produktionsverhältnissen angepasst sind. Die Vorgaben für die Unterstützung der Pflanzenzüchtung sind in der «Strategie Pflanzenzüchtung Schweiz 
2050» festgehalten, die klare Vorgaben für die öffentlich-rechtlich finanzierte Pflanzenzüchtung in der Schweiz gibt. Im Kern wird dadurch die Forschung der Agroscope und diejenige von insgesamt zehn unabhängigen Akteuren koordiniert und mitfinanziert. Damit soll sichergestellt werden, dass hochwertige Sorten entwickelt werden, die gut an die vielfältigen Bedingungen der Schweiz angepasst sind und damit einen Beitrag leisten können an eine nachhaltige Land- und Ernährungswirtschaft.

Im Tierbereich ist das Ziel, eine eigenständige Tierzucht in der Schweiz zu erhalten. Deshalb unterstützt der Bund Zuchtorganisationen mit Beiträgen für tierzüchterische Massnahmen wie Herdebuchführung, Leistungsprüfungen und Zuchtwertschätzungen sowie für Projekte im Zusammenhang mit der Erhaltung der einheimischen Rassenvielfalt. Ausserdem ist das BLW zuständig für die Anerkennung von Zuchtorganisationen und die Bewirtschaftung von Zollkontingenten für Zuchttiere und Rindersperma. Ausserdem muss der Bund ein Gestüt halten. Insgesamt werden für die Förderung und Erhaltung der inländischen Pflanzen- und Tierzucht 38,5 Mio. CHF eingesetzt.

In Bezug auf die genetischen Ressourcen ist einerseits die Erhaltung von alten Tierrassen ein wichtiger Aspekt. Um künftig auf neue Rahmenbedingungen wie z.B. neue Krankheiten reagieren zu können, unterstützt der Bund Massnahmen zur Erhaltung und Förderung gefährdeter Nutztierrassen mit Schweizer Ursprung. International engagiert sich das BLW im «European Regional Focal Point», ein internationales Gremium zur Erhaltung von Nutztierrassen. Im Bereich der Pflanzen betreibt das BLW eine nationale Genbank für die Erhaltung pflanzengenetischen Ressourcen für Ernährung und Landwirtschaft. Auch hier ist es das Ziel, die genetische Vielfalt zu erhalten, um in Zukunft auf veränderte Rahmenbedingungen, wie beispielsweise durch den Klimawandel, reagieren zu können.

\subsubsection{Pflanzenschutz und Produktionsmittel}

Im Bereich des Pflanzenschutzes sollen einerseits die Einführung und Verbreitung von Krankheiten und Schädlingen bei den Pflanzen vermieden werden. Dazu gehört beispielsweise die Bekämpfung von gemeingefährlichen Krankheiten und Schädlingen wie Feuerbrand oder Ambrosia. Andererseits wird der Einsatz von Pflanzenschutzmitteln (PSM) reguliert. Als wichtige Instrumente dazu zählen das Bewilligungsverfahren für PSM, die Überprüfung der zugelassenen Anwendungsvorschriften, aber auch Massnahmen zur Verminderung der Anwendung und zur Risikoreduktion von PSM. Dazu hat der Bund einen Aktionsplan Pflanzenschutzmittel entwickelt, der eine strategische Vorgabe für die Entwicklung der Massnahmen vorgibt. Ziel des Aktionsplans ist es, die Risiken im Einsatz von PSM zu halbieren und Alternativen zum chemischen Pflanzenschutz zu fördern. Die Entscheidung über die Zulassung von Pflanzenschutzmitteln lag bis 2021 beim Bundesamt für Landwirtschaft. Ab 2022 wird 
das Bundesamt für Lebensmittelsicherheit und Veterinärwesen über diese Zulassung entscheiden.

Der 7. Titel des Landwirtschaftsgesetzes befasst sich ausserdem mit der Zulassung für landwirtschaftliche Produktionsmittel. Dazu gehören neben den Pflanzenschutzmitteln auch Dünger, Saatgut und Futtermittel. Im Kern geht es dabei um die Lebensmittelsicherheit. Produktionsmittel dürfen nur angewendet werden, wenn sie eine gesetzeskonforme Produktion von Lebensmitteln erlauben und keine Nebenwirkungen auf Menschen, Tiere und Umwelt haben. Der Bund darf auch vorsorglich, d.h. ohne umfassende wissenschaftliche Risikobeurteilung, die Verwendung von Produktionsmitteln einschränken.

\subsection{Bundesgesetz über das bäuerliche Bodenrecht}

Das Bundesgesetz über das bäuerliche Bodenrecht (BGBB) enthält sechs Titel. Diese haben den folgenden Inhalt.

- $\quad$ Allgemeine Bestimmungen: Neben dem Zweck, dem Gegenstand und dem Geltungsbereich werden im ersten Titel einige zentrale Begriffe definiert, die für das Gesetz eine wichtige Rolle spielen: das landwirtschaftliche Grundstück, das landwirtschaftliche Gewerbe, das parzellenweise verpachtete Gewerbe, der Selbstbewirtschafter und der Ertragswert.

- $\quad$ Privatrechtliche Beschränkungen: Dieser Titel handelt von den privatrechtlichen Beschränkungen des Rechtsverkehrs mit landwirtschaftlichen Gewerben und Grundstücken. Mit anderen Worten wird der Verkehr der genannten Objekte innerhalb der Familie geregelt. Der Staat wird in solchen Fällen nicht aktiv. Es ist Sache der einzelnen Personen, die Rechte, die ihnen nach dem Gesetz zustehen, nötigenfalls mithilfe des zuständigen Zivilgerichts, selber wahrzunehmen.

- Öffentlich-rechtliche Beschränkungen: Dieser Titel beinhaltet die öffentlichrechtlichen Beschränkungen, die in Zusammenhang mit dem Verkehr mit landwirtschaftlichen Gewerben und Grundstücken stehen. Er befasst sich mit familienexternen Handänderungen. Gegenstände dieses Titels sind das Zerstückelungsverbot, das Realteilungsverbot für ganze Gewerbe und die Bestimmungen bezüglich des Erwerbsbewilligungsverfahrens.

- Massnahmen zur Verhütung der Überschuldung: Beim 4. Titel wird grundsätzlich das Prinzip der Belehnungsgrenze bzw. Belastungsgrenze aufgenommen, welche die Verschuldung von landwirtschaftlichen Grundstücken beschränkt.

Die Titel 5 und 6 befassen sich mit Verfahren, Rechtsschutz und der Zuständigkeit der Kantone und des Bundes. 
Der Zweck des BGBB ist es, das bäuerliche Grundeigentum zu fördern und dadurch bodenbewirtschaftende Familienbetriebe zu erhalten. Das Gesetz sieht ausserdem vor, dass die Stellung des Selbstbewirtschafters gestärkt und übersetzte Preise für den landwirtschaftlichen Boden bekämpft werden. Ein Selbstbewirtschafter ist, wer den landwirtschaftlichen Boden selber bearbeitet und, wenn es sich um ein landwirtschaftliches Gewerbe handelt, dieses persönlich leitet. Der Kauf von Flächen in der Landwirtschaftszone ist damit auf aktive Bäuerinnen und Bauern beschränkt. Die Bewilligung zum Erwerb eines landwirtschaftlichen Gewerbes oder Grundstücks wird nämlich verweigert, wenn der Erwerber nicht Selbstbewirtschafter ist oder wenn für den Kauf ein übersetzter Preis vereinbart wurde. Die entsprechende Bewilligung muss vom zuständigen Kanton erteilt werden.

Das BGBB kennt dabei zwei Rechtsobjekte: jenes des landwirtschaftlichen Grundstücks und des landwirtschaftlichen Gewerbes. Als wesentliches Steuerungsinstrument dient der Begriff des landwirtschaftlichen Gewerbes. Als landwirtschaftliches Gewerbe gilt eine Gesamtheit von landwirtschaftlichen Grundstücken, Bauten und Anlagen, die als Grundlage der landwirtschaftlichen Produktion dient und zu deren Bewirtschaftung, wenn sie landesüblich ist, mindestens eine Standardarbeitskraft nötig ist. Die Standardarbeitskraft (SAK) ist eine Einheit zur standardisierten Bemessung der Betriebsgrösse. Die zur Berechnung verwendeten Faktoren basieren auf arbeitswirtschaftlichen Grundlagen, d.h. einem Faktor pro GVE oder pro Hektare. Die SAK wird nicht nur im BGBB, sondern in verschiedenen Politikbereichen als Steuerungsinstrument genutzt, insbesondere beim Bezug von Direktzahlungen sowie bei der Gewährung von Strukturverbesserungsmassnahmen und von sozialen Begleitmassnahmen. Der Bund nutzt die Standardarbeitskraft, um verschiedene Politikmassnahmen auf Betriebe mit einer Mindestgrösse zuzuschneiden (Tailoring). Insbesondere im Bereich des BGBB und der Strukturverbesserungsmassnahmen soll die SAK ein objektives Kriterium sein, gezielt diejenigen Betriebe zu fördern, die den Bäuerinnen und Bauern eine langfristige Existenz bieten können.

Die zentralen Mechanismen im BGBB sind, dass 1), wenn ein Gewerbe vorliegt, jeder selbstbewirtschaftende und dafür geeignete Erbe in der Erbteilung die ungeteilte Zuweisung des Gewerbes zum Ertragswert verlangen kann, 2) für landwirtschaftliche Gewerbe das Verbot der Realteilung gilt, d.h., dass von einem Gewerbe nicht einzelne Grundstücke abgetrennt werden dürfen, und 3) der Pächter an einem zu veräussernden landwirtschaftlichen Grundstück ein Vorkaufsrecht hat, wenn er Eigentümer eines landwirtschaftlichen Gewerbes ist. Schliesslich kann sich, wer als Erbe bereits Eigentümer eines Gewerbes ist, zudem ein in der Erbschaft befindendes und in der Nähe des Gewerbes liegendes landwirtschaftliches Grundstück zum doppelten Ertragswert zuweisen lassen. Verwandte haben zudem unter gewissen Voraussetzungen ein Vorkaufsrecht an einem Gewerbe oder Grundstück. 
Umgekehrt bedeutet das, dass die zentralen Mechanismen des BGBB, die erbrechtliche Übernahme des Betriebs zum Ertragswert, das Vorkaufsrecht des Pächters und der Verwandten und das Realteilungsverbot für Betriebe mit weniger als einer SAK nicht gelten. Die Kantone können allerdings den Grenzwert für die Gewerbegrenze auf 0,6 SAK senken. Insgesamt zehn Kantone (Appenzell Innerrhoden, Bern, Freiburg, Genf, Glarus, Jura, Luzern, Solothurn, Tessin und Waadt) machten seit 2008 von diesem Recht Gebrauch, mit Bezug zum damaligen Grenzwert von 0,75 SAK. Die Kantone Bern, Glarus und Luzern stuften die kantonalen Gewerbegrenzen nach Zonen ab. Für Betriebe aus den Hügel- und Bergzonen gilt ein tieferer SAK-Grenzwert als für Betriebe im Talgebiet.

Die zentrale Verbindung zwischen den Zielen «bäuerliches Grundeigentum fördern und namentlich bodenbewirtschaftende Familienbetriebe erhalten» und der "Zuweisung zum Ertragswert» ist, dass Bäuerinnen und Bauern die Betriebe übernehmen können, ohne sich (stark) verschulden zu müssen. Damit soll die finanzielle Tragbarkeit der Bauernbetriebe sichergestellt werden. Das Ziel des Schutzes vor Überschuldung stand schon am Ursprung der Einführung des Ertragswertprinzips im Jahr 1907. Das Ertragswertprinzip ermöglicht eine Verhütung von Überzahlungen landwirtschaftlicher Heimwesen durch eine möglichst objektive Bewertung der Liegenschaften.

Der Ertragswert entspricht dem Kapital, das mit dem Ertrag eines landwirtschaftlichen Gewerbes oder Grundstücks bei landesüblicher Bewirtschaftung zum durchschnittlichen Zinssatz verzinst werden kann. Eine näherungsweise Berechnung sieht folgendermassen aus: Angenommen, der Ertrag einer landesüblichen Bewirtschaftung für eine Hektare entspricht dem Pachtzins, dann muss dieser Betrag mit dem durchschnittlichen Zinssatz kapitalisiert werden. Liegt der Pachtpreis bei 600 Franken pro Hektare und der Zinssatz bei $3 \%$, dann käme der Ertragswert bei 20000 Franken zu liegen (600/0.03 = 20000 Fr.). Nehmen wir an, dass die gleiche Fläche für 4 Franken pro Quadratmeter verkauft werden könnte $(4$ x $10000=40000$ Fr. $)$, dann ist der Ertragswert halb so hoch wie der Verkehrswert dieser Fläche. Der Ertragswert wird mittels einer Schätzungsanleitung festgelegt. Diese muss regelmässig ergänzt und weiterentwickelt werden, damit sie den aktuellen wirtschaftlichen und technischen Entwicklungen Rechnung trägt. 2018 wurde eine neue Anleitung eingeführt, welche jeder Schätzung zugrunde liegt. Das heisst, für die Ermittlung des Ertragswertes muss eine gründliche Besichtigung der landwirtschaftlichen Gewerbe oder Grundstücke durchgeführt und jedes Gebäude und jede Fläche nach ihrem langjährigen mittleren Ertrag beurteilt werden. Seit 2018 ist eine neue Schätzungsanleitung in Kraft. Diese berücksichtigt insbesondere, dass die Nutzungsdauer der Gebäude beschränkt ist.

Analog zu den Strukturverbesserungen verfolgt der Gesetzgeber auch im Bereich des BGBB strukturelle Ziele. Mit der Gewerbegrenze definiert der Bund ein «Pforte in die Landwirtschaft» bzw. ein «Eingangstor in den Beruf Landwirt». Mit der Wahl der 
Grösse dieses Eingangstors kann die Politik den Strukturwandel der landwirtschaftlichen Betriebe mit beeinflussen. Die Vorteile einer Übernahme zum Ertragswert, d.h. die Möglichkeit der Übernahme zu günstigen Konditionen, sollen dabei in erster Linie denjenigen Betrieben zugestanden werden, die längerfristig eine Existenzgrundlage bieten. Förderungswürdige Betriebe sind damit in erster Linie Haupterwerbsbetriebe. In den früheren Bestimmungen war man noch von einer Unterscheidung in Haupt- und Nebenerwerbsbetriebe ausgegangen. Diese Unterscheidung erfolgt im bestehenden Recht über die SAK, ohne dies explizit auszudrücken.

Wie bei den Strukturverbesserungen wird beim Gewerbe die landwirtschaftliche Substanz ebenfalls als zentrales Förderungskriterium gesehen. Bei der Feststellung des Gewerbes soll nicht ausschliesslich auf die bestehenden Verhältnisse abgestellt werden, sondern darauf, was in Zukunft sinnvoll ist und bei realistischer Betrachtung erreicht werden kann. Gemäss der Formulierung einer «landesüblichen Bewirtschaftung» verlangt das BGBB eine objektive Beurteilung.

Die Gewerbegrenze sollte damit im bestehenden Recht ein rein objektives Mass für die minimale Betriebsgrösse eines Gewerbes sein. Die Gewerbegrenze und damit die Zuweisung zum Ertragswert hat somit auch ein strukturelles Ziel. Mit der Aufgabe von Nebenerwerbsbetrieben mit geringer kernlandwirtschaftlicher Substanz, d.h. der pflanzlichen und tierischen Produktion und deren Verarbeitung, welche die Gewerbegrenze nicht erreichen, sollen Flächen für Betriebe mit einer ausreichenden kernlandwirtschaftlichen Substanz und einer längerfristigen Existenzfähigkeit frei werden. Die Gewerbegrenze soll explizit den Strukturwandel nicht hemmen. Dadurch wird gleichzeitig der innerlandwirtschaftlichen Konkurrenz entgegengewirkt, weil bei einem stagnierenden Produktionswert der Schweizer Landwirtschaft das Einkommen pro Betrieb weniger stark unter Druck gerät. Im Gegensatz zur Anwendung im Bereich der Strukturverbesserungen erfolgt bei der Gewerbefeststellung keine einzelbetriebliche Prüfung der wirtschaftlichen Existenzfähigkeit des Betriebs. Sofern der Bewirtschafter die grundlegenden SAK-Anforderungen zur ortsüblichen Bewirtschaftung erfüllt, den Betrieb selber bewirtschaften will und dafür geeignet ist, kann er den Betrieb bzw. die Produktionsstätte zum Ertragswert übernehmen.

Auch das Realteilungsverbot hat eine strukturelle Komponente. Die Bewirtschaftungseinheiten sollen eine rationelle landwirtschaftliche Nutzung ermöglichen. Eine Zerstückelung von Parzellen würde diesem Ziel entgegenwirken. Zudem wird damit sichergestellt, dass landwirtschaftlich genutzte Parzellen nicht so weit zerstückelt werden, dass sie nicht mehr unter die Regelungen des BGBB fallen. Neben diesen strukturellen Zielen (Erhöhung der Flächenmobilität, weniger Konkurrenz unter Bauern) beabsichtigt der Gesetzgeber mit der Anwendung der Gewerbegrenze, auch familieninterne Ungerechtigkeiten zu verhindern. Unter der Voraussetzung einer geringen kernlandwirtschaftlichen Substanz besteht die Gefahr, dass die landwirtschaftliche Produktion nach der Übernahme zum Ertragswert extensiviert wird. Die 
Miterben würden dadurch benachteiligt. Zu bemerken ist, dass eine lebzeitige Hofübergabe keiner Zustimmung der Miterben bedarf und daher auch zum Ertragswert erfolgen kann, wenn es sich beim Betrieb nicht um ein landwirtschaftliches Gewerbe handelt. Der Übernehmer muss aber gewärtigen, dass durch die nicht gerechtfertigte Übernahme zum Ertragswert eine lebzeitige Zuwendung vorliegt und die Miterben im späteren Erbfall die erbrechtliche Ausgleichung oder Herabsetzung verlangen können.

Das Boden- und Pachtrecht in der Schweizer Landwirtschaft ist komplex. So ist es nicht verwunderlich, dass die Kommentare zum Bundesgesetz über das bäuerliche Bodenrecht, herausgegeben vom Schweizerischen Bauernverband, fast 1000 Seiten umfassen. Trotz dieser Komplexität lässt sich die Gesetzgebung auf eine einfache Zielstellung zurückführen: Die landwirtschaftlichen Flächen sollen Familienbetrieben gehören und von diesen auch bewirtschaftet werden. Dies wird mit den bestehenden Regelungen erreicht. Der Nachteil ist, dass die Ein- und Austrittshürden in und aus der Landwirtschaft hoch sind.

\section{ZUSAMMENFASSUNG}

Der Grundstein für die Agrargesetzgebung in der Schweiz sind die beiden Verfassungsartikel 104 und 104a. Diese setzen die übergeordneten Ziele und Rahmenbedingungen im Schweizer Landwirtschaftsgesetz, dem Bundesgesetz zum bäuerlichen Bodenrecht und weiteren für die Landwirtschaft relevanten Gebieten wie dem Pachtrecht, dem Raumplanungsrecht oder dem Umweltschutz. Die Kernelemente des Landwirtschaftsgesetzes sind die Steuerungen im Bereich Produktion und Absatz, die Direktzahlungen und Strukturverbesserungen sowie die Regelungen in den Bereichen Pflanzen- und Tierzucht, Pflanzenschutz und Produktionsmittel. Im Bundesgesetz über das bäuerliche Bodenrecht wird die Erhaltung einer bäuerlichen Landwirtschaft gefördert und sichergestellt, dass landwirtschaftliche Flächen nur von Bäuerinnen und Bauern erworben werden können. 


\section{Beurteilung der Agrargesetzgebung}

Aus der Kombination der Beurteilungskriterien der Agrarpolitik im Kapitel 6 und der Beschreibung der Ausgestaltung der Schweizer Agrarpolitik im Kapitel 9 lässt sich eine agrarökonomische Beurteilung der Instrumente und Massnahmen vornehmen. Eine solche Beurteilung beruht üblicherweise auf einer Evaluation der entsprechenden Massnahmen. In diesem Kapitel werden zuerst Grundbegriffe der Evaluation von Politikmassnahmen eingeführt. Anschliessend werden bestehende, wissenschaftliche Evaluationen der Schweizer Agrarpolitik zusammengefasst. Diese Zusammenfassung hat keinen Anspruch auf Vollständigkeit. Das Ziel ist es aber, den Leserinnen und Lesern eine generelle Übersicht zur Relevanz, Wirksamkeit und Effizienz der bestehenden Agrarpolitik zu vermitteln.

\subsection{Grundbegriffe der Evaluation von Politikmassnahmen}

\subsubsection{Beurteilungskriterien}

Um ein agrarpolitisches Element zu beurteilen, werden üblicherweise drei Kriterien hinzugezogen. Erstens soll ein agrarpolitisches Instrument das Kriterium der Relevanz erfüllen. Die Massnahme oder das agrarpolitische Instrument soll zur Erreichung eines übergeordneten politischen Ziels, das meist in der Verfassung festgelegt wird, beitragen. Zweitens muss ein geeignetes Instrument Effektivität gewährleisten. Eine Massnahme muss also effektiv ein zu definierendes Sachziel unterstützen. Drittens hängt eine positive Beurteilung auch vom Kriterium der Effizienz ab. Effizienz ist dann gegeben, wenn die definierten Ziele durch diese Massnahme mit möglichst tiefen Kosten erreicht werden können. In der Beurteilung von agrarpolitischen Instrumenten stehen diese drei Kriterien im Vordergrund. Wie die Dimensionen der Ausgestaltung von agrarpolitischen Instrumenten im vorhergehenden Kapitel gezeigt haben, gibt es aber noch weitere Kriterien, die einer ganzheitlichen Beurteilung von Politikmassnahmen einfliessen können.

An dieser Stelle sollen vier weitere Kriterien erwähnt werden. Diese sind nicht abschliessend (für eine vollständige Übersicht siehe Ingold et al., 2016). Erstens ist es wichtig, dass eine Massnahme auch eine gewisse Kohärenz zu anderen Massnahmen aufweist. Sie sollte z.B. nicht einem anderen Instrument entgegenwirken oder sogar widersprechen. Zweitens sollte eine agrarpolitische Massnahme immer auch auf ihre Eignung in der Umsetzung geprüft werden. Eine geringe Akzeptanz der Politikadres- 
saten oder hohe Transaktionskosten vermindern die Eignung eines Instruments. Drittens muss ein Instrument angemessen bzw. verhältnismässig sein. Die angestrebte Massnahme sollte nicht zu komplex sein oder die Kosten einseitig einem Akteur aufdrängen. Viertens sollte eine agrarpolitische Massnahme auch nachhaltig sein, in dem Sinne, dass sie langfristig finanzierbar ist und institutionell, d.h. innerhalb der Verwaltung verankert werden kann.

\subsubsection{Wirkungsmodell zur Evaluation von agrarpolitischen Mass- nahmen}

Zur Evaluation einer Politikmassnahme werden die oben aufgeführten Kriterien oft mittels eines Wirkungsmodells zusammengefasst (siehe auch Box 14). Dieses erlaubt es, die Kriterien einzeln oder kombiniert bzw. aus einer Pro- oder Retrospektive zur Beurteilung von agrarpolitischen Massnahmen beizuziehen. In der Evaluation von agrarpolitischen Massnahmen in der Schweiz bezieht sich z.B. das Bundesamt für Landwirtschaft systematisch auf ein Wirkungsmodell.

Dazu werden im Wirkungsmodell in einem ersten Schritt die übergeordneten politischen Ziele, meistens aus dem Art. 104 BV, und die Sachziele, üblicherweise aus dem LwG, der einzelnen Massnahmen definiert. Je grösser der Beitrag eines Sachziels zum übergeordneten politischen Ziel ist, desto höher ist die Relevanz der entsprechenden Massnahme. Anschliessend wird festgelegt, was die Verhaltensziele sein sollen, d.h., welches Verhalten der Landwirte durch die Umsetzung der Massnahme erwartet wird. Mit dem erwünschten Verhalten der Landwirte sind spezifische landwirtschaftliche Aktivitäten verbunden. Diese Aktivitäten führen zu Outputs oder Leistungen und sollen dadurch zu Auswirkungen, einem Outcome oder Effekt, im Sinne der Sachziele führen. In einer gesamtheitlichen Analyse der Wirkung, d.h. auch unter der Berücksichtigung von Umwelt- oder Marktentwicklungen, wird der Impact einer Massnahme beurteilt. Es ist wichtig zu beachten, dass Aktivitäten, Leistungen (Outputs) und Auswirkungen bzw. Outcomes nicht zwangsläufig gleich sind.

Am Beispiel der Zahlung für Biodiversitätsförderflächen können die Begrifflichkeiten exemplarisch dargestellt werden (siehe Kapitel 9.1 für eine exakte Beschreibung der Massnahmen). Die Formulierung in der Bundesverfassung besagt, dass die Landwirtschaft einen Beitrag dazu leisten soll, die natürlichen Lebensgrundlagen zu erhalten (übergeordnetes Ziel). Im Landwirtschaftsgesetz Art. 73 ist festgehalten, dass zur Förderung und Erhaltung der Biodiversität (Sachziel) Biodiversitätsbeiträge ausgerichtet werden. Das erwartete Verhalten ist, dass die Landwirte vermehrt Rücksicht auf die Biodiversität nehmen und Aktivitäten umsetzen, die diese fördern, d.h. Biodiversitätsförderflächen generieren. In der Umsetzung ihrer Aktivitäten generieren die Bäuerinnen und Bauern einen Output oder eine Leistung. Das bedeutet, dass sie beispielsweise eine Wiese extensiv nutzen (z.B. erster Schnittzeitpunkt nach Mitte Juni 
und kein Düngereinsatz) oder Hochstammobstbäume pflanzen. Die extensiven Wiesen oder die Hochstammobstbäume sind die Outputs oder Leistungen. Die Auswirkung müsste sein, dass die Biodiversität in der landwirtschaftlichen Kulturlandschaft zunimmt oder zumindest nicht abnimmt. Letzteres folgt aber nicht automatisch aus der Aktivität bzw. der Leistung, weil die Wirksamkeit (der Effekt) eben auch von anderen Faktoren, wie beispielsweise Umweltbedingungen oder Massnahmen, die ausserhalb der Landwirtschaft liegen, beeinflusst werden kann. Tatsächlich nimmt die Biodiversität in der Schweizer Kulturlandschaft ab, obwohl die Landwirte ja einen Output bzw. eine Leistung liefern - in Form der extensiv bewirtschafteten Flächen. Bezieht man schliesslich auch andere Faktoren und Politiken in die Beurteilung mit ein, kann der Impact einer Politikmassnahme beurteilt werden. Im Kontext der Biodiversitätsbeiträge würde sich dann die Frage stellen, ob die Zahlungen für Biodiversitätsförderflächen der Intensivierung der Landwirtschaft entgegenwirken und damit den Rückgang der Biodiversität ganz allgemein aufhalten können.

Auf der Ebene des Vollzugs beurteilt ein Wirkungsmodell das Verhältnis zwischen den staatlichen Inputs, z.B. den Direktzahlungen, und dem dadurch erzielten Output bzw. den erbrachten Leistungen, z.B. der ha Biodiversitätsförderflächen. Das Verhältnis zwischen Kosten der Steuerzahler und Landwirte und Auswirkung wird als Kosteneffektivität (Cost-Benefit) bezeichnet. Das Verhältnis zwischen Input und Output kann als Kosteneffizienz betrachtet werden (Cost-Efficiency). Während die Kosteneffektivität etwas darüber aussagt, ob sich eine staatliche Massnahme überhaupt lohnt, kann die Kosteneffizienz dazu verwendet werden, unterschiedliche Massnahmen miteinander zu vergleichen und das kostengünstigste auszuwählen. An dieser Stelle ist es wichtig, festzuhalten, dass diese Begriffe in unterschiedlichen wissenschaftlichen Disziplinen auch unterschiedlich verwendet werden können. So begreifen die Politikwissenschaften beispielsweise das Instrument an und für sich, also z.B. die Direktzahlung, als Output des Politikprozesses (Ingold et al., 2016). Deshalb ist es zentral, dass eine Evaluation immer in ein Wirkungsmodell eingebettet ist und die Begriffe klar definiert sind.

Die unterschiedlichen Stufen eines Wirkungsmodells gelten nicht nur für agrarpolitische Massnahmen im Umweltbereich, sondern können beispielsweise auch für ein Instrument des Aussenhandels definiert werden. Die Erhöhung des einheimischen Produzentenpreises wäre in diesem Fall der Output der Anwendung eines Zollkontingents. Der Beitrag zu den Einkommen der inländischen Bäuerinnen und Bauern durch das Zollkontingent ist der Outcome. Der Impact schliesslich bezieht auch die Auswirkungen auf Dritte wie beispielsweise Konsumenten, die einen höheren Preis für Lebensmittel bezahlen, in die Beurteilung mit ein. 
Box 14 Beispielhaftes Wirkungsmodell zur Evaluation von Biodiversitätsbeiträgen BFF

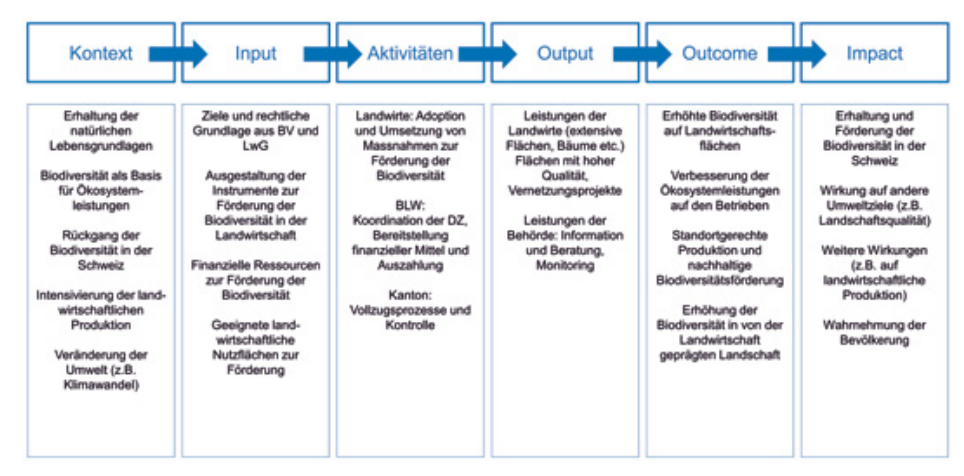

Die Grundlage für die Evaluation von agrarpolitischen Massnahmen ist ein Wirkungsmodell. Dies spezifiziert zuerst den Kontext bzw. beschreibt das Problem, welches mit einer Politikmassnahme gelöst werden soll. Die Biodiversitätsbeiträge haben zum Ziel, die natürlichen Lebensgrundlagen zu erhalten, damit wichtige Ökosystemleistungen gewährleistet werden können. Der Input umfasst die gesetzlichen Grundlagen und die zur Verfügung stehenden finanziellen und personellen Ressourcen. In Bezug auf Biodiversitätsbeiträge liefern das LwG bzw. die Direktzahlungsverordnung die rechtliche Grundlage. Insgesamt stellt der Bund jährlich etwas mehr als 400 Mio. CHF für die Massnahmen BFF 1, BFF 2 (Qualitätszahlung) und BFF Vernetzung zur Verfügung. Die Kantone kontrollieren die Umsetzung der Massnahmen und koordinieren die Vernetzungsprojekte. Die Aktivitäten beziehen sich auf das gewünschte Verhalten von den von der Politikmassnahme betroffenen Akteuren. Im Kontext der Biodiversitätsbeiträge sind dies in erster Linie die Landwirte, welche naturnahe Produktionsmassnahmen zur Förderung der Biodiversität umsetzen sollen. Der Output der Politikmassnahme sind die Leistungen der Politikadressaten. Das heisst im Falle der BFF, wie viele Flächen extensiv bewirtschaftet oder wie viele Hochstammobstbäume gepflegt werden. Der Outcome beschreibt den Effekt auf das anvisierte Ziel. Im Fall der BFF ist das die Erhöhung der Biodiversität auf den Landwirtschaftsflächen und der von der Landwirtschaft geprägten Landschaft. Der Impact schliesslich umfasst auch gewollte und ungewollte Nebeneffekte der Politik wie beispielsweise den Beitrag zur Landschaftspflege oder den Einfluss auf die landwirtschaftliche Rohstoffproduktion. 
Das Bundesamt für Landwirtschaft lässt jährlich mehrere Politikevaluationen durchführen, welche auf einem solchen oder ähnlichen Wirkungsmodell aufbauen. Diese werden von der Agroscope oder privaten Unternehmensberatungen durchgeführt. Die Resultate werden auf der Webseite des Bundesamts publiziert.

Die Unterscheidung in Output, Outcome und Impact mag akademisch wirken. Ein Blick auf die bestehenden agrarpolitischen Debatten zeigt jedoch, dass diese Unterscheidung keineswegs irrelevant ist. In der Diskussion zur Umweltwirkung stellt sich z.B. der Bauernverband auf den Standpunkt, dass man die Ziele in Bezug auf den Output erreicht und damit weitere Massnahmen im Umweltbereich obsolet seien. Umweltschutzverbände stellen sich dagegen auf den Standpunkt, dass die Landwirtschaft die formulierten Ziele in Bezug auf die Outcomes nicht erreicht, da die Biodiversität in der Schweiz nach wie vor zurückgeht. Ohne eine konzeptionelle Klärung mithilfe eines Wirkungsmodells können beide Seiten behaupten, recht zu haben.

Erst die Einordnung in ein Wirkungsmodell und die Beurteilung des Impacts lassen eine vollumfängliche Evaluation der Massnahmen zu. Das Problem dabei ist, dass sich selten sämtliche direkten und indirekten Wirkungen in der Realität tatsächlich messen lassen. In der Evaluation von agrarpolitischen Massnahmen oder Instrumenten aus einer rationalen Perspektive, prospektiv oder retrospektiv, bestehen fünf zentrale Herausforderungen (Engel, 2016):

1) Unklare Kausalitäten. Nicht in jedem Fall sind die Kausalzusammenhänge zwischen Verhalten, Aktivitäten und Auswirkung klar und messbar nachvollziehbar. Es ist in diesem Fall auch schwierig, eine Systemgrenze klar festzulegen, weil ökonomische, ökologische und soziale Wirkungen ineinander verwoben sind. Es lässt sich daher auch nur schwer ein statistisches Modell entwickeln, welches die Kausalitäten klar voneinander trennt.

2) Interaktionen und Messgrössen. Die Kombination von Effekten verschiedener Massnahmen lässt sich in der Analyse oft nicht trennen (siehe Abschnitt zu Jointness). Dies macht es schwierig, einen Indikator festzulegen, der die Zielgrösse exakt misst.

3) Fehlende kontrafaktische Position. In vielen Fällen gibt es keine Vergleichsgruppe oder Situation, in der festgestellt werden könnte, wie sich der Outcome ohne agrarpolitische Massnahme entwickelt hätte. Es können sich daher nicht beobachtbare Faktoren auf den Output auswirken, welche die effektive Wirkung schmälern. Ein Schlüsselbegriff dabei ist auch der sogenannte Selektionsfehler (Selection bias), der entsteht, wenn in der Analyse einer Massnahme die Eigenschaften von Landwirtschaftsbetrieben nicht zufällig verteilt sind. Konkret könnte 
es sein, dass beispielsweise die Wirkung einer Agrarumweltmassnahme nur in Betrieben nachgewiesen werden kann, in denen der Betriebsleiter eine positive Einstellung zum Umweltschutz hat. Wenn dieser Aspekt in der Analyse nicht berücksichtigt wird, dann kann der Effekt fälschlicherweise der Massnahme zugewiesen werden, obwohl eigentlich die Umwelteinstellung die ursächliche Wirkung war.

4) Basiseffekt. In der Agrarpolitik spielen der Agrarstrukturwandel und andere dynamische Prozesse eine wichtige Rolle. Viele agrarpolitische Effekte werden durch dynamische Entwicklungen überlagert und lassen sich nur schwer über die Zeit isoliert beurteilen.

5) Referenzsituation. Das Ziel einer agrarpolitischen Massnahme kann es sein, einen negativen Trend aufzuhalten. Wenn in der Evaluation kein positiver Zusammenhang zwischen Massnahme und Auswirkung festgestellt werden kann, ist das manchmal darauf zurückzuführen, dass das Ziel lediglich vorsah, den Zustand nicht zu verschlechtern. Der Referenzpunkt wäre dann eine kontrafaktische Position in der Zukunft und nicht die Ausgangslage zum Zeitpunkt der Einführung der Massnahme.

Die Evaluation einer agrarpolitischen Massnahme ist dadurch alles andere als trivial. Tatsächlich gibt es nur sehr wenige Studien, die auf alle diese möglichen Herausforderungen eingehen. Wichtig ist aber, diese zu kennen und die Evaluationen mit Blick auf die theoretischen und methodischen Einschränkungen zu beurteilen, die sich aus der Komplexität der Zusammenhänge zwangsläufig ergeben.

\subsection{Evaluationen der Schweizer Agrarpolitik}

Gemäss der Datenbank ARAMIS, welche die vom Bund (mit-)finanzierten Evaluationsund Forschungsprojekte dokumentiert, sind seit der Einführung der neuen Agrarpolitik 1999 mehr als hundert Projekte mit Bezug zur Landwirtschaft durchgeführt worden. Darüber hinaus haben auch die ETH, Universitäten und Fachhochschulen diverse wissenschaftsbasierte Evaluationen der Schweizer Agrarpolitik vorgenommen. Das Ziel ist an dieser Stelle nicht, sämtliche dieser Evaluation zu beschreiben. Mit einem Fokus auf diejenigen Studien, deren Resultate auch in wissenschaftlichen Zeitschriften veröffentlicht wurden, werden aber die wichtigsten Erkenntnisse mit Blick auf die Aspekte Relevanz, Effektivität und Effizienz zusammengefasst. Die Unterkapitel folgen der Reihenfolge des Landwirtschaftsgesetzes.

\subsubsection{Rahmenbedingungen für die Produktion}

Ein zentrales und äusserst relevantes Element in der Schweizer Agrarpolitik ist der Grenzschutz. Eine umfassende Darstellung des Grenzschutzes und deren Wirkung wurde letztmals von der OECD durchgeführt (Gray et al., 2017). Dabei zeigt sich, dass die Instrumente des Grenzschutzes ein effektives Mittel sind, um die Produzenten- 
preise in der Schweiz zu stützen. Spezifische Evaluationen der Zollkontingente im Bereich Fleisch und Gemüse (Loi et al., 2016), aber auch der Milch (Hillen und von Cramon-Taubadel, 2019) zeigen, dass der Stützungseffekt über verschiedene Instrumente und Sektoren effektiv ist. Auch die saisonalen Zollkontingente schützen die inländische Produktion sehr effektiv vor ausländischen Importen (Hillen, 2019). Die agrarökonomische Kritik am Grenzschutz folgt aber aus Effizienzüberlegungen. Dazu gehört einerseits, dass der Grenzschutz per se viel weniger zielgerichtet ist als andere Instrumente der Schweizer Agrarpolitik (Gray et al., 2017). Andererseits führt dies dazu, dass der Grenzschutz sich auf die gesamte Wertschöpfungskette auswirkt und nicht nur auf die Produzentenpreise (Loi et al., 2016). Dadurch entstehen Renten, die nicht zu einem konkreten Ziel der Agrarpolitik gehören und ohne den Grenzschutz nicht entstehen würden (Hillen, 2019). Damit verbunden ist in erster Linie ein Konsumentenrentenverlust, der aus einer wohlfahrtsökonomischen Perspektive problematisch ist. Ausserdem zeigen frühere Studien, dass entlang der Wertschöpfungskette eine asymmetrische Preistransmission stattfindet, z.B. im Schweinemarkt (Abdulai, 2002). Das bedeutet, dass Erhöhungen schneller an die Konsumenten weitergegeben werden als Senkungen der Produzentenpreise. Dies impliziert eine gewisse Marktmacht, welche die Akteure in der Wertschöpfungskette ausnutzen können. Allerdings zeigen neuere Untersuchungen im Bereich Weizen (Esposti und Listorti, 2018) und Milch (Hillen, 2021), dass diese asymmetrische Weitergabe der Produzentenpreise nicht mehr oder nur für wenige Produktkategorien beobachtet werden können. Das deutet darauf hin, dass die Branchenorganisationen eine effektive Steuerung der Preise für inländische Nahrungsmittel erreichen. Allerdings bleibt die Analyse von Preisen und Renten über die gesamte Wertschöpfungskette hinweg eine grosse Herausforderung. Der Grund dafür ist, dass wichtige Elemente wie beispielsweise die Kostenstruktur oder die Margen entlang der Wertschöpfungskette nicht empirisch beobachtet, sondern höchstens über beobachtbare Preise angenähert werden können. Das schränkt die Möglichkeiten zur Evaluation des Grenzschutzes ein. Neueste Evaluationen deuten aber weiterhin auf einen grossen Einfluss des Detailhandels auf die hohen Konsumentenpreise in der Schweiz hin (Bokusheva et al., 2019; Mann et al., 2019), was mit den theoretischen Überlegungen zur Marktmacht übereinstimmt.

Ein wichtiges Element im Kontext der Rahmenproduktion von Milch ist das Freihandelsabkommen mit der EU im Bereich des Käses. Die erste Evaluation des Abkommens zeigte, dass das Volumen von Importen und Exporten erwartungsgemäss zugenommen hat und dass der Wert der Exporte höher ist als der Wert der Importe (BAK Basel 2012). Neuere Evaluationen zeigen, dass der Effekt auf die Schweizer Käseproduktion als moderat einzustufen ist (Kohler, 2016). Es muss allerdings auch hier der Einwand gemacht werden, dass aufgrund der Komplexität des Milchmarktes (z.B. "gelbe» und «weisse» Linie) eine eindeutige Identifikation der Auswirkungen 
nur bedingt möglich ist. Ausserdem wurden die Auswirkungen auf die Produzentenpreise über die Verkäsungszulage und die Zulage für silofreie Milch abgeschwächt. Evaluationen zeigen, dass diese Instrumente effektiv die Produzentenpreise stützten. Der Effekt unterschied sich aber stark zwischen Käsen unterschiedlicher Produktionsherkunft. Gewerblicher Käse wie Gruyère profitiert mehr als industriell hergestellter Käse (Finger et al., 2017). Insgesamt muss aber auch hier darauf hingewiesen werden, dass die effektive Stützung der Produzentenpreise mit einer tieferen internationalen Wettbewerbsfähigkeit und höheren Konsumentenpreisen verbunden ist (Gray et al., 2017).

Weitere Freihandelsabkommen hätten diesbezüglich einen grossen Einfluss auf die Schweizer Agrarpolitik. Die OECD sieht in der Aufgabe des Grenzschutzes und eines Umbaus der landwirtschaftlichen Stützung eine grosse Chance, um die Effizienz der Schweizer Agrarpolitik zu erhöhen (Gray et al., 2017). Reformbedarf bestünde auch in Bezug auf die Vereinfachung und die Transparenz des Systems (Widmer, 2017). Expertenanalysen legen ebenfalls nahe, dass die Wettbewerbsfähigkeit in der Landwirtschaft durch Freihandel erhöht und die Marktmacht gewisser Akteure reduziert werden könnte (Wey und Gösser, 2019). Ex ante Analysen von Marktöffnungsszenarien zeigen aber, dass dadurch das sektorale Einkommen in der Schweizer Landwirtschaft in den nächsten zehn Jahren um einen Drittel sinken würde (Mack et al., 2017). Damit wäre ein erheblicher Effekt auf die landwirtschaftlichen Einkommen und den Strukturwandel in der Schweizer Landwirtschaft zu erwarten (Huber und Häberli, 2010). Wichtig ist dabei zu bemerken, dass die Wirkung solcher Abkommen stark von deren Ausgestaltung abhängt. So könnte beispielsweise das Freihandelsabkommen mit den Mercosur-Staaten (Argentinien, Brasilien, Paraguay, Uruguay) (Portmann und Ritzel, 2020) in Abhängigkeit der Zugeständnisse nur kleine Effekte auf den Agrarsektor haben. Gleichzeitig könnten die Abkommen aber auch zu negativen Umwelteffekten führen, insbesondere in Bezug auf den Wasserverbrauch und den Biodiversitätsverlust (Alig et al., 2019).

Agrarökonomische Evaluationen in Bezug auf andere Massnahmen im Kontext der Produktion und des Absatzes bestehen nur wenige. Eine zentrale Herausforderung ist dabei, wie sich die Wirkungen von Massnahmen identifizieren lassen können. So zeigt z.B. die Evaluation der Absatzförderung, dass die Umsetzung des Instruments mit den agrarpolitischen Vorgaben übereinstimmt. Eine effektive Messung der Wirkung ist aber nicht möglich und mögliche Empfehlungen beziehen sich auf Effizienzüberlegungen zur Optimierung des Instruments, aber weniger auf die Frage, ob das Instrument tatsächlich zu den Zielen der Agrarpolitik beiträgt (Rieder et al., 2015). Auch im Bereich der Ursprungsbezeichnungen und der Labels gibt es, über die Beschreibung der Regulierungen (Aouinaït et al., 2020) und einzelner Fallstudien hinaus (Vandecandelaere et al., 2018), wenig Informationen. 


\subsubsection{Direktzahlungssystem}

Durch die hohen Bundesausgaben für Direktzahlungen kommt diesen eine hohe Relevanz für die Erreichung der agrarpolitischen Ziele zu. Ein zentraler Aspekt ist dabei, dass die Zahlungen an den ökologischen Leistungsnachweis geknüpft sind. Ganz allgemein zeigen Evaluationen, dass mit dem ökologischen Leistungsnachweis die Umweltbelastung in der Schweizer Landwirtschaft abgenommen hat und positive Umweltaspekte gestärkt wurden (Herzog et al., 2017). So wurde beispielsweise die diffuse Verschmutzung von Grund- und Oberflächengewässern mit Stickstoff und Phosphor reduziert (Herzog et al., 2008; Kupper et al., 2015). Gleichzeitig nahmen die Flächen zur Förderung der Biodiversität zu, mit positiven Effekten auf Pflanzen (Herzog et al., 2005; Knop et al., 2006; Aviron et al., 2008; Kampmann et al., 2008; Kampmann et al., 2012), Arthropoden (Aviron et al., 2008; Albrecht et al., 2010), Säugetiere (Zellweger-Fischer et al., 2011) und Vögel (Birrer et al., 2007; Zingg et al., 2018; Zingg et al., 2019). Ausserdem haben Blühstreifen und andere ökologische Elemente einen positiven Einfluss auf das Management von Krankheiten im Ackerbau (Tschumi et al., 2015; Tschumi et al., 2016; Herzog et al., 2017). Auch die Treibhausgasemissionen sind aufgrund der Einführung des Direktzahlungssystems zurückgegangen (Leifeld und Fuhrer, 2005) und die Produktionsformen mit zu erwartenden positiven Auswirkungen auf Umwelt und Tiere wie die biologische Landwirtschaft (Nemecek et al., 2011; Zimmermann et al., 2011; Schader et al., 2013; Necpalova et al., 2018) oder die Teilnahme an den Tierwohlprogrammen haben zugenommen (Schmid und Lehmann, 2000; Vogeler, 2017). Insgesamt hat die Implementierung und Durchsetzung des ökologischen Leistungsnachweises einen positiven Effekt auf die Umwelt bewirkt. Simulationsergebnisse zeigen auch, dass gewisse Verschärfungen des ökologischen Leistungsnachweises unter gewissen Annahmen nur zu wenig Einkommensverlusten in der Landwirtschaft führen würden und gleichzeitig die Umweltindikatoren verbessern könnten (Schmidt et al., 2019; Bystricky et al., 2020).

Trotz dieser positiven Entwicklung bleibt die Wirkung des Direktzahlungssystems begrenzt. Die Umweltziele des Bundes für die Schweizer Landwirtschaft werden nicht oder nur in einzelnen Teilaspekten tatsächlich erreicht. Dafür ausschlaggebend sind in erster Linie zwei Aspekte.

Einerseits besteht eine Diskrepanz zwischen dem Output bzw. der Leistung von Direktzahlungen und dem Outcome, d.h. der tatsächlich messbaren Veränderung von Umweltindikatoren. Das führt dazu, dass Direktzahlungen trotz räumlicher Zielgenauigkeit (Targeting) und einem hohen Grad an Abstufung (Tailoring) immer noch ineffektiv sind. Die Erhöhung der Biodiversitätsförderflächen beispielsweise hat zwar einen Einfluss auf das Vorhandensein von Arten (siehe oben), den eigentlichen Trend des Rückgangs der Biodiversität in den Agrarlandschaften konnte der Anstieg der extensiv genutzten Flächen jedoch nicht stoppen (Fontana et al., 2019). Ein anderes 
Beispiel ist das GMF-Programm. Die Evaluation zeigt, dass viele Betriebe zwar mitmachen, die effektive Reduktion des Stickstoffs in der Produktion von Milch und Fleisch jedoch gering ist. Viele Betriebe mussten ihre Produktion mit der Teilnahme nicht oder nur geringfügig umstellen (tiefe Additionalität). Dadurch entstanden Mitnahmeeffekte für die Betriebe im Tal- wie im Berggebiet, welche die Effizienz der Massnahme reduzierte (Mack und Huber, 2017). Auch in der Evaluation der Landschaftsqualitätsbeiträge wurde gezeigt, dass die Einführung des Programms zu Mitnahmeeffekten bei den teilnehmenden Bauern führte (Steiger et al., 2016).

Andererseits hat die differenzierte Ausgestaltung des Direktzahlungssystems über mehr als hundert Massnahmen dazu geführt, dass zwischen den einzelnen Anreizen neue Spannungsfelder entstehen. Hohe Versorgungssicherheitsbeiträge beispielsweise konkurrieren direkt die mehr ökologisch ausgerichteten Direktzahlungen im Talgebiet (Huber und Lehmann, 2010). Im Berggebiet können hingegen Zahlungen, welche Flächen in der Produktion halten, ökologische Leistungen fördern, weil die Flächenanteile über den ökologischen Leistungsnachweis miteinander gekoppelt sind (Huber et al., 2017b). Neueste Untersuchungen zeigen auch, dass mit der Kombination von input- und outcome-orientierten Massnahmen, wie im Schweizer BFFProgramm, die Mitnahmeeffekte zunehmen (Wüpper und Huber, 2021), das Programm aber trotzdem einen ökonomischen Mehrwert bietet. Schliesslich zeigte sich, dass mit der Komplexität der Massnahmen die kantonalen Verwaltungen einen wichtigen Einfluss auf die Teilnahme bei diesen Programmen haben (Mack et al., 2020).

Zusammenfassend lässt sich sagen, dass das Direktzahlungssystem in der Schweiz einen Beitrag zu mehr ökologischen Leistungen leistet, die Effizienz aber deutlich verbessert werden könnte, damit die Umweltziele erreicht werden können. Eine höhere Effizienz würde es ermöglichen, Zahlungen in gewissen Kategorien einzusparen und diese Gelder in anderen Programmen mit höherer Additivität einzusetzen. Zentral ist dabei auch, dass die Programme von den Landwirten akzeptiert werden. Das Verständnis für eine bestimmte Massnahme reduziert nämlich den wahrgenommenen administrativen Aufwand der Bäuerinnen und Bauern (Ritzel et al., 2020; Mack et al., 2021). Wichtig für die Akzeptanz der Massnahmen ist aber auch, dass das Direktzahlungssystem insgesamt einkommenswirksam ist. So zeigt die Evaluation der Versorgungssicherheitsbeiträge beispielsweise, dass diese Beiträge das Einkommen der Bäuerinnen und Bauern erhöhen (Möhring und Mann, 2020). Ausserdem zeigen Analysen der Einkommensverteilung, dass die Einführung des Direktzahlungssystems die Ungleichheit in den landwirtschaftlichen Einkommen reduzierte (El Benni et al., 2012). Diese Erhöhung und Stabilisierung der Einkommen war ein explizites Ziel bei der Einführung der Direktzahlungen. 
Es besteht daher auch eine gewisse Güterabwägung zwischen der Effektivität und der Effizienz von Massnahmen. Aus der Perspektive der Effizienz wäre es einerseits wünschenswert, wenn die Abgeltung der ökologischen Leistungen den Opportunitätskosten derer Erbringung entsprechen würde (Huber et al., 2017a). Andererseits entstehen durch die Erbringung von ökologischen Leistungen auch Einkommen. Während die WTO vorschreibt, dass die Zahlung von ökologischen Leistungen über den Erbringungskosten nicht erlaubt seien, stellt sich aus einer gesamtheitlichen Sicht die Frage, weshalb Bäuerinnen und Bauern nicht auch mit der Produktion von Umweltgütern Gewinne machen dürfen (Mann, 2006a). Ein wichtiger Aspekt dabei ist, dass die Gelder für die Direktzahlungen in der Schweizer Agrarpolitik nicht knapp zu sein scheinen. Die Bereitschaft der Bevölkerung, des Gesetzgebers und der Regierung, einen relativ hohen Anteil des Budgets für die Landwirtschaft auszugeben, führt dazu, dass auch ineffiziente Zahlungen als Teil des Systems akzeptiert werden (siehe dazu auch die Ausführungen im nächsten Kapitel 11).

\subsubsection{Weitere Massnahmen}

Während es zahlreiche Studien zur Wirkung des Grenzschutzes und der Direktzahlungen gibt, sind agrarökonomische Analysen anderer Massnahmen seltener. Insbesondere bei Strukturverbesserungsmassnahmen und der Wirkung des Bundesgesetzes über das bäuerliche Bodenrecht gibt es nur wenige Evaluationen. Das hat sicherlich mit der Tatsache zu tun, dass die Auswirkungen dieser Massnahmen, wie beispielsweise der landwirtschaftliche Strukturwandel, immer im Kontext verschiedener ökonomischer, sozialer und politischer Faktoren beurteilt werden müssen und weniger auf eine bestimmte Massnahme zurückgeführt werden können (Ecoplan und HAFL, 2016; Neuenfeldt et al., 2018). Evaluationen zur Betriebshilfe und den Investitionshilfen zeigen aber, dass auch diese Instrumente im Kern effektiv sind, d.h., sie erreichen die von der Agrarpolitik gestellten Sachziele (Flury und Peter, 2011; Flury et al., 2012; EFK, 2015). Kritik an den Strukturverbesserungsmassnahmen bezieht sich daher eher auf die politischen Ziele als darauf, ob diese auch erreicht werden. Auch die Evaluation der Projekte regionaler Entwicklung (Flury et al., 2017) zeigt, dass das Instrument «Projekte regionaler Entwicklung» Wertschöpfungspotenziale in ländlichen Gebieten gezielt unterstützen kann. Für diese Instrumente stellt sich weniger die Frage, ob sie die Ziele tatsächlich erreichen, sondern ob die Umsetzung effizient und das eigentliche politische Ziel relevant ist.

Insgesamt zeigt der Überblick zu den Evaluationen von agrarpolitischen Instrumenten, dass viele agrarpolitische Ziele erreicht werden, die Effizienz dieser Massnahmen aber oft kritisch zu beurteilen ist. Eine zentrale Herausforderung ist sicher die Kohärenz zwischen den unterschiedlichen Programmen, aber auch zwischen den einzelnen Instrumenten. Hier werden auch in Zukunft wissenschaftlich fundierte Evaluationen notwendig sein. 


\section{ZUSAMMENFASSUNG}

Die Wirkung der Ausgestaltung von agrarpolitischen Massnahmen und Instrumenten sollte immer auf Relevanz, Effektivität und Effizienz überprüft werden. Dazu wird ein sogenanntes Wirkungsmodell verwendet. In einem Wirkungsmodell ist es zentral, zwischen Input, Output, Outcome und Impact zu unterscheiden. Gerade in der Agrarpolitik spielen diese unterschiedlichen Ebenen eine wichtige Rolle, weil die Zusammenhänge zwischen Aktivitäten der Landwirtschaft und deren Wirkungen komplex und oft schwer messbar sind und damit nicht in jedem Fall klar identifizierbar sind. Evaluationen der agrarpolitischen Instrumente in der Schweiz zeigen, dass die Effektivität der Massnahmen (die Erreichung eines Sachziels) hoch ist. Gleichzeitig ist aber die Effizienz (die Leistungen oder Outcomes gemessen an den Kosten, die sie verursachen) in den meisten Fällen tief. Die Evaluationen zeigen ausserdem, dass eine Weiterentwicklung des Systems, neben einer Verbesserung der Effizienz, auf die Kohärenz der unterschiedlichen Massnahmen und Programme Rücksicht nehmen sollte.

\section{WEITERFÜHRENDE LITERATUR}

Eine theoretische Einordnung der unterschiedlichen politischen Eingriffe und deren Auswirkungen aus einer agrarökonomischen Perspektive ist im Beitrag von Rausser und Goodhue im Handbook of Agricultural Economics beschrieben: «Public policy: Its many analytical dimensions».

Das Lehrbuch Politikevaluation. Eine Einführung von Fritz Sager, Susanne Hadorn, Andreas Balthasar und Céline Mavrot führt in die Grundlagen der Politikevaluation ein.

Die Grundlagen für die Evaluation von Politikprogrammen und Instrumenten im Bereich der Agrarumweltpolitik sind im Lehrbuch von Karin Ingold, Eva Lieberherr, Isabelle Schläpfer, Kathrin Steinmann und Willi Zimmermann mit dem Titel Umweltpolitik der Schweiz konzise zusammengefasst. 


\section{Institutionen und Prozesse der Agrar- politik}

\section{READERS' GUIDE}

Die bestehende Ausgestaltung der Agrarpolitik zeigt, dass sich die Umsetzung von agrarpolitischen Instrumenten nicht zwangs/äufig an den wohlfahrtstheoretischen Überlegungen ausrichtet. Dies hat einerseits damit zu tun, dass die Umsetzung von Instrumenten in der realen Welt sehr viel komplexer ist als in den theoretischen Wohlfahrtsanalysen angenommen. Andererseits hängt die Umsetzung agrarpolitischer Instrumente von den politischen Institutionen (z.B. Regierungssystem) und den politischen Verfahren (z.B. Gesetzgebungsprozesse) ab. Theoretisch lassen sich diese beiden Bereiche in die Polity und Politics einteilen (Kapitel 2). Dieses Kapitel beleuchtet die Institutionen und Prozesse der Agrarpolitik aus einer theoretischen Perspektive und beschreibt anschliessend die bestehenden Prozesse in der heutigen Gestaltung der Schweizer Agrarpolitik. Im theoretischen Teil fokussiert das Kapitel auf die Konzepte und Theorien der neuen politischen Ökonomie. Diese liefern Argumente zur Erklärung von unterschiedlichen Agrarpolitiken. Anschliessend wird der Politikzyklus vorgestellt. Aus der Kombination dieser beiden Grundlagenkapitel werden dann in den beiden folgenden Kapiteln die bestehenden agrarpolitischen Prozesse in der Schweiz beschrieben, zuerst aus einer institutionellen und anschliessend aus einer politischen Netzwerk-Perspektive. Das Ziel dieses Kapitels ist es, dass die Leserinnen und Leser einen Einblick in die agrarökonomischen Grundlagen zur Erklärung von Politikprozessen erhalten. Darüber hinaus sollen sie die aktuellen Prozesse und Abläufe in der Entstehung der Schweizer Agrarpolitik kennenlernen.

\subsection{Neue politische Ökonomie}

Die neue politische Ökonomie, je nach Strömung auch als Public oder Social Choice betitelt, beschäftigt sich mit der Interpretation politischer Entscheidungen durch die Anwendung ökonomischer Konzepte (Mueller, 2015). Die Grundannahme ist, dass politische Massnahmen und deren Umsetzungen, d.h. die Policy, stark von der politischen Machtverteilung und der politischen Struktur der Institutionen geformt werden (Rausser und Swinnen, 2011). Diese Betrachtung schliesst dabei nicht nur insti- 
tutionalisierte politische Akteure, z.B. die Regierung, Parlament, Parteien, Bundesämter etc., in den Entscheidungsprozess mit ein, sondern berücksichtigt auch die Macht anderer Akteure, wie beispielsweise der politisch aktiven Interessengruppen, die durch Lobbying Einfluss nehmen (Swinnen, 2010). Zudem geht mit der ökonomischen Betrachtung einher, dass den Individuen ein Streben nach einer individuellen Nutzenmaximierung und ein rationales Verhalten zugeschrieben wird. In Analogie zur Kernfrage der Ökonomie - wie lassen sich knappe Ressourcen so einsetzen, dass Wohlfahrt entsteht - lautet die Frage in der neuen politischen Ökonomie, wie sich begrenzte politische Ressourcen so einsetzen lassen, dass Wohlfahrt politisch gefördert wird. Die neue politische Ökonomie geht dabei davon aus, dass sich auch in der Politik "Marktmechanismen» etablieren, welche das Angebot von politischen Leistungen durch Politiker und Verwaltung mit der Nachfrage nach diesen Leistungen von Interessenvertretern und Wählern zusammenbringen (Henrichsmeyer und Witzke, 1994). In den folgenden Unterkapiteln werden die Grundüberlegungen der politischen Ökonomie dargelegt.

\subsubsection{Grundlagen der neuen politischen Ökonomie: Wohlfahrt und Verhalten}

Die Kernfrage der politischen Institutionen und Prozesse ist: Wer soll die Regeln in der Agrarpolitik festlegen? In einer Demokratie wird grundsätzlich davon ausgegangen, dass gesellschaftspolitische Aufgaben durch die Regierung übernommen werden und diese im Interesse der Regierten, d.h. der Bevölkerung, handelt. Diese Betrachtung beruht auf zwei Annahmen: 1) Es gibt ein definiertes Gemeinwohl. 2) Die Politiker haben von sich aus das Interesse, dieses Gemeinwohl anzustreben (Kirchgässner, 2010). Auf die Schweizer Agrarpolitik angewendet würde dies bedeuten, dass es eine Form der Landwirtschaftspolitik gäbe, die alle Interessen berücksichtigt und das Wohl aller Akteure, Konsumenten, Produzenten etc., gleichermassen anstrebt.

Diese beiden Annahmen werden mit den Konzepten der neuen politischen Ökonomie kritisch hinterfragt. Konkret untersucht die neue politische Ökonomie die theoretischen Grundlagen der sozialen Wohlfahrt und analysiert das Verhalten von politischen Akteuren in einem demokratischen System. Auf diese beiden Aspekte wird im Folgenden kurz eingegangen.

\subsubsection{Soziale Wohlfahrtsfunktion}

Um eine soziale Wohlfahrtsfunktion bestimmen zu können, ist es notwendig, die Präferenzen einer Gesellschaft abzubilden. Basierend auf der liberalen Position wird davon ausgegangen, dass ausschliesslich Individuen ihre eigenen Präferenzen kennen. Um auf eine allgemeine gesellschaftliche Präferenz zu schliessen, ist es deshalb notwendig, diese individuellen Präferenzen zu aggregieren (Varian, 2016). Zwei wichtige 
Theoreme der neuen politischen Ökonomie postulieren allerdings, dass eine konsistente Aggregation individueller Präferenzen nicht möglich ist. Das sogenannte Condorcet-Paradox und das Arrow-Theorem offenbaren, dass eine einfache Mehrheitsregel zu einem Zirkelschluss führen kann und dass es kein politisches Abstimmungsverfahren gibt, das zu einer gesellschaftlichen Präferenzordnung führt und gleichzeitig für eine Demokratie fundamentale Bedingungen erfüllt (Sen, 2018). Die Implikation ist, dass es keine Agrarpolitik geben kann, welche die soziale Wohlfahrt maximieren und gleichzeitig demokratisch sein kann.

Die Folgerung daraus ist nicht, dass die Agrarpolitik diktatorisch bestimmt werden sollte. Das Theorem legt aber den Grundstein dazu, zu erkennen, dass es wichtig ist, die Prozesse, die zur Einschränkung der individuellen Präferenzordnungen oder der Abweichungen vom Paretooptimum führen, zu kennen und in der Interpretation bzw. Entwicklung der Agrarpolitik zu berücksichtigen. Mit anderen Worten, es gibt keine perfekte Regel, mit welcher die Präferenzen von Individuen aggregiert werden können, und damit auch keine soziale Wohlfahrtsfunktion (Varian, 2016). Unter gewissen Bedingungen können aber Präferenzen geordnet werden und daraus Entscheidungsregeln hergeleitet werden. Beispielsweise können durch Rangierung von Alternativen, Verhandlungslösungen oder sogenannten Maxmin-Ordnungen theoretisch optimale und faire Entscheidungen entstehen (Sen, 2018).

\subsubsection{Verhalten von politischen Akteuren}

Neben der Erkenntnis, dass die Prozesse genauso wichtig sind wie die normativen Grundlagen der Agrarpolitik, wird in der neuen politischen Ökonomie davon ausgegangen, dass sich die involvierten Akteure in individuellen Entscheidungssituationen befinden und grundsätzlich versuchen, ihren Nutzen innerhalb der gegebenen Restriktionen zu maximieren. Mit anderen Worten, die neue politische Ökonomie geht davon aus, dass sich die Akteure rational im Sinne der Nutzenmaximierung unter Unsicherheit verhalten (Kirchgässner, 2010). Im Zentrum der Analysen stehen dabei die die Produzenten, die Konsumenten und die Steuerzahler. Dazu kommen die vor- und nachgelagerten Industrien und Verbände, aber auch die Umweltlobbys oder andere Interessengruppen (Swinnen, 2018).

Eine zentrale Rolle nehmen die Politikerinnen und Politiker selbst ein. Eine politökonomische Grundlage für das Verhalten von Politikerinnen und Politikern ist das sogenannte Medianwählertheorem (Downs, 1957): Werden die Präferenzen der Wähler auf einer eindimensionalen Skala angeordnet, befindet sich der Medianwähler genau in der Mitte dieser Skala. Das Theorem besagt, dass sich politische Entscheidungen an den Präferenzen des Medianwählers orientieren. Die ökonomische Theorie der Demokratie geht nun davon aus, dass die Politiker ihren Nutzen, bestehend aus Macht, Ansehen und Einkommen des politischen Amtes, zu maximieren versuchen. Da die Maximierung dieser Punkte durch eine hohe Anzahl Wählerstimmen erreicht 
werden kann, versucht der Politiker eben diese zu maximieren (Stimmenmaximierung). Weil es sich gemäss der Theorie auch bei den Wählern um rationale Nutzenmaximierer handelt, wählen diese diejenigen Vertreter, die ihre Interessen am ehesten vertreten. Der Politiker oder die Politikerin gewinnt dann am meisten Stimmen, wenn er oder sie sich dem Medianwähler anpasst, weil die Anzahl der Wähler, die sich mit diesem identifizieren können, maximal ist. Der ökonomischen Theorie der Demokratie folgend agieren Politiker also nicht, um die soziale Wohlfahrt zu steigern, sondern sie orientieren sich in ihrem Handeln an den sich daraus ergebenden Chancen, wiedergewählt zu werden.

Das Verhalten der politischen Akteure kann somit direkt mit demjenigen von wirtschaftlichen Unternehmern verglichen werden. Diese Überlegungen gelten aber nicht nur für den Politiker und den Wähler, sondern auch für andere Akteure der Agrarpolitik wie beispielsweise die Interessengruppen oder die Verwaltung. Die neue politische Ökonomie unterstellt auch diesen Akteuren ein nutzenmaximierendes Verhalten durch Lobbying und Rent-Seeking. Wenn man davon ausgeht, dass die Akteure ihren Nutzen maximieren und es keine übergeordnete Zielfunktion gibt, d.h. eine soziale Wohlfahrtsfunktion, dann kann man den agrarpolitischen Prozess auch als Markt sehen: Die Politiker und die Administration sind Anbieter von politischen Massnahmen, z.B. einem Zoll, und die Bevölkerung bzw. Interessengruppen, welche die Bevölkerung repräsentieren, sind die Nachfrager von politischen Massnahmen. Im folgenden Unterkapitel wird dieses Konzept des politischen Marktes am Beispiel des Protektionismus illustriert.

\subsubsection{Der politische Markt am Beispiel des Protektionismus}

Zur Erinnerung: Die neue politische Ökonomie basiert auf den Grundsätzen, dass es keine soziale Wohlfahrt gibt, die allen Präferenzen der Bürgerinnen und Bürger gerecht werden kann, und dass sämtliche Akteure im politischen System sich rational verhalten, d.h., sie versuchen ihren Nutzen zu maximieren, der beispielsweise darin bestehen kann, Einkommen zu sichern, wiedergewählt zu werden oder Einfluss und Macht zu gewinnen. Aus diesen Überlegungen heraus lässt sich, analog zur Marktwirtschaft, auch ein politischer Markt definieren, in welchem sich Angebot, d.h. die Leistungen der Politik, und Nachfrage, d.h. die Präferenzen der Bürgerinnen und Bürgern, gegenüberstehen.

Dies lässt sich am Beispiel des Agrarprotektionismus gut illustrieren. Obwohl durch die Wirtschaftstheorie gezeigt wird, dass Zölle einer Ökonomie eigentlich schaden (siehe Kapitel 6.3), sind sie nach wie vor allgegenwärtig. Deren Existenz kann durch politische Marktüberlegungen erklärt werden. Die Angebotsseite besteht in diesem Beispiel aus dem Gesetzgeber und der öffentlichen Verwaltung, welche die Zölle etabliert und verwaltet. Die Nachfrage nach Handelsprotektionismus ist wiederum abhängig von den Kosten und dem Nutzen der politischen Aktivität von Befürwortern 
des Protektionismus. Theoretisch wird die Höhe des Zolls dort zu liegen kommen, wo die marginalen Kosten des Angebotes und der marginale Nutzen gleich sind. Wenn vom abnehmenden Grenznutzen der politischen Aktivität ausgegangen wird, bedeutet dies, dass der politische Einsatz zugunsten hoher Zölle auch höhere Kosten mit sich bringt, als dies für tiefere Zölle der Fall wäre. Wenn schon Zölle bestehen, wird zusätzlicher Protektionismus folglich «politisch» teurer (Frey, 1985).

Zudem gibt es auch Interessengruppen, die eine Liberalisierung unterstützen. Befürworter und Gegner von Protektionismus versuchen dabei den Einsatz der Opposition zu antizipieren. Je nach angenommenem Ressourceneinsatz der Gegenseite wird der entsprechende politische Akteur seine Ressourcen (Arbeit und Kapital) dazu verwenden, um seinen eigenen Standpunkt durchzubringen. Dieser Prozess führt zu einem Gleichgewicht (Cournout-Nash-Gleichgewicht), in welchem keine Seite mehr einen Anreiz hat, ihre politische Aktivität zu verändern (Frey, 1985). Eine Erhöhung der Aktivität würde unnötige Kosten verursachen, während eine Verringerung der Aktivität der Gegenseite zugutekommen würde. Volkswirtschaftlich betrachtet werden für dieses «Rent-Seeking» Ressourcen eingesetzt, die zur Herstellung von Gütern eingesetzt werden könnten. Die neue politische Ökonomie argumentiert, dass dadurch zusätzliche Wohlfahrtsverluste entstehen, die über die theoretischen ökonomischen Verluste hinausgehen. Rent-Seeking bedeutet somit nichts anderes, als dass politische Interessenvertreter versuchen, Programme oder Instrumente einzuführen, die eine ökonomische Rente generieren, von der ihre Klientel profitieren kann. RentSeeking wird aber natürlich von verschiedenen Akteuren praktiziert. Das alleine erklärt den Hang zum Protektionismus noch nicht.

Eine zentrale Rolle im politischen Markt kommt den Gewinnern und Verlierern zu (Rodrik, 2018). Bei einer politischen Veränderung mit dem Ausmass einer Liberalisierung des Handels kann jeweils eine Gewinner- und eine Verliererseite ausgemacht werden. Bei einer Öffnung des Agrarmarktes würden beispielsweise die landwirtschaftlichen Produzenten im Inland als Verlierer resultieren, während die Konsumenten von den wohl tieferen Preisen profitieren könnten. Die Verlierer des Zollabbaus werden aber nicht notwendigerweise oder im vollen Ausmass für ihre Verluste entschädigt. Diese wehren sich folglich gegen diese staatliche Handlung. Sie versuchen, die (Produzenten-)Rente zu erhalten. Wenn eine Liberalisierung des Handels nun mehr Verlierer als Gewinner produziert, ist eine protektionistische Haltung das Resultat. Weiter haben die Gewinner einer politischen Veränderung einen weniger starken Problemdruck als die Verlierer und somit weniger Anreize, sich zu informieren und politisch zu engagieren. Gleichzeitig handelt es sich bei den Gewinnern oft um eine heterogene und grosse Gruppe, die weniger gut organisierbar ist und Platz für Trittbrettfahrer lässt (siehe auch das nächste Kapitel zum kollektiven Handeln). 
Im Fall der Agrarpolitik spielt auch die politische Vertretung der Verliererseite im Parlament eine wichtige Rolle. Sie kann beispielsweise durch Stimmenabtausch ihre politische Agenda durchbringen. Dies ist allerdings nur dann möglich, wenn die Verliererseite auch über eine mächtige Verhandlungsposition verfügt. Dies ist in der Schweiz mit dem starken Einfluss des Schweizerischen Bauernverbands (SBV) möglich. Die politische Vertretung der Landwirtschaft schafft es aktuell immer wieder, ihre protektionistischen Haltungen in bilateralen Freihandelsabkommen einzubringen.

Im internationalen politischen Kontext lassen sich die Verhandlungspositionen zum Thema Freihandel durch ein Gefangenendilemma annähern (Varian, 2016). In einem Gefangenendilemma wird eine Situation beschrieben, in der sich die Akteure für oder gegen die Kooperation mit dem jeweils anderen Akteur entscheiden müssen. Den höchsten Nutzen würden beide Akteure dann erreichen, wenn sie miteinander kooperierten. Die höchsten Verluste werden allerdings dann erzielt, wenn sich ein Akteur für die Kooperation entscheidet, während der andere nicht auf Kooperation setzt. Weil die beiden Akteure ihre Entscheidungen gleichzeitig treffen müssen, werden sich also beide Akteure dafür entscheiden, nicht zu kooperieren und somit den minimalen Nutzen umgehen. Im Falle des Protektionismus führt dies dazu, dass die Staaten sich immer für eine protektionistische Strategie entscheiden werden. Diese unvorteilhafte Situation kann allerdings durch eine verbesserte Kommunikation und Kooperation der Akteure umgangen werden. Diese Kooperationsmöglichkeit bietet sich im Falle des Freihandels in der WTO. Die fehlende Koordination zwischen den einzelnen Ländern ist dann auch eine zentrale Herausforderung für die Weiterentwicklung der Agrarhandelspolitik (Scott, 2017).

Wie gut erklären aber diese theoretischen Ansätze den "Agrarprotektionismus» tatsächlich? Empirische Analysen haben verschiedene Faktoren isolieren können, die stark mit dem Auftreten von Protektionismus korrelieren (De Gorter und Swinnen, 2002). Dabei hat sich gezeigt, dass neben einer tiefen Produktivität und tiefen landwirtschaftlichen Einkommen in erster Linie diejenigen Faktoren für einen verstärkten Protektionismus ausschlaggebend sind, welche für eine schwache wirtschaftliche Position der Landwirtschaft im Vergleich zu den anderen Sektoren stehen. Der Protektionismus ist höher in Ländern mit einem tiefen Anteil der landwirtschaftlichen Wertschöpfung am Bruttosozialprodukt oder in Ländern mit sinkendem Anteil der Haushaltsausgaben an Ausgaben für Lebensmittel. Diese Beobachtungen können das Modell des politischen Marktes des Protektionismus und des Cournout-Nash-Gleichgewichts theoretisch untermauern. Die landwirtschaftlichen Akteure erleben in diesen Ländern bereits höhere Kosten als der Rest der Gesellschaft. Der Nutzen aus dem Protektionismus und dementsprechend aus dem politischen Einsatz dafür ist folglich viel höher, als wenn die Landwirtschaft eine gute wirtschaftliche Stellung innehat. 
Aus Sicht der Schweizer Landwirtschaft können die theoretischen Grundlagen wohl viele Aspekte der Agrarpolitik mit erklären. Die Schweiz ist geradezu ein Paradebeispiel für die Erklärung von Protektionismus: Importland, tiefer Anteil an der Wertschöpfung, tiefe Haushaltsausgaben etc. Gleichzeitig ist es aus Sicht der neuen politischen Ökonomie wichtig zu erkennen, dass sich hinter dem hehren Ziel einer nachhaltigen Landwirtschaft handfeste finanzielle Interessen und Machtverhältnisse verbergen.

\subsubsection{Kollektives Handeln}

Die Überlegungen der neuen politischen Ökonomie verdeutlichen, dass neben den Politikerinnen und Politikern auch weitere Akteure einen wichtigen Einfluss auf die Agrargesetzgebung haben. Dazu gehören insbesondere die Interessenvertreter wie Bauernverbände oder Umweltschutzorganisationen. Aus einer ökonomischen Betrachtungsweise heraus stellt sich die Frage, wie die Interessen des Einzelnen in den politischen Markt eingebracht werden können.

Eine wichtige Grundlage zur Beantwortung dieser Frage ist die Theorie des kollektiven Handelns (Collective Action Theory) von Mancur Olson (Olson, 1979). Gemäss dieser Theorie lässt sich der unterschiedliche Einfluss, den Interessengruppen auf das politische Basissystem ausüben, oft durch die unterschiedlichen Gruppengrössen und die Homogenität der Interessen der verschiedenen Gruppierungen erklären. Kleine Gruppen mit homogenen Interessen müssen nach dieser Theorie weniger Kosten aufbringen, um ein öffentliches Gut, hier die Interessenvertretung einer Branche, bereitstellen zu können. Dies ermöglicht es ihnen, auch mit geringen finanziellen Ressourcen politischen Einfluss auszuüben. Grosse Interessengruppen hingegen haben diesen Vorteil nicht, da bei ihnen zwingend Organisationskosten anfallen. Der Zusammenschluss grösserer Gruppen resultiert daher nicht zwingend in stärkerer politischer Einflussnahme, obwohl mehr Mitglieder und potenziell mehr finanzielle Ressourcen zur Verfügung stehen. Ein Beispiel dafür sind die Konsumenten. Diese lassen sich nur schwer organisieren, weil ihre Interessen äusserst heterogen sind.

Neben einer kleinen Gruppengrösse und homogener Interessen gibt es weitere Faktoren, die einen Zusammenschluss trotz anfallender Organisationskosten ermöglichen können. So haben zum einen bestehende Organisationen tiefere Einstiegskosten (Frey, 1985). Verfügt eine bestehende Organisation über ein funktionierendes Netzwerk in Wirtschaft und Verwaltung, kann es mit wenig Ressourcen viele Mitglieder vertreten. Zum anderen lassen sich die Interessen von unorganisierten Gruppen mit einer hohen Anzahl Mitgliedern, sogenannte latente Gruppen, durch selektive Anreize aggregieren. Der selektive Anreiz, der für die Interessensorganisation in grossen Gruppen notwendig ist, kann dabei aus einer Belohnung oder aus einem Zwang bestehen, muss sich allerdings immer auf das rational handelnde Individuum beziehen (Hendrikse, 2003). 


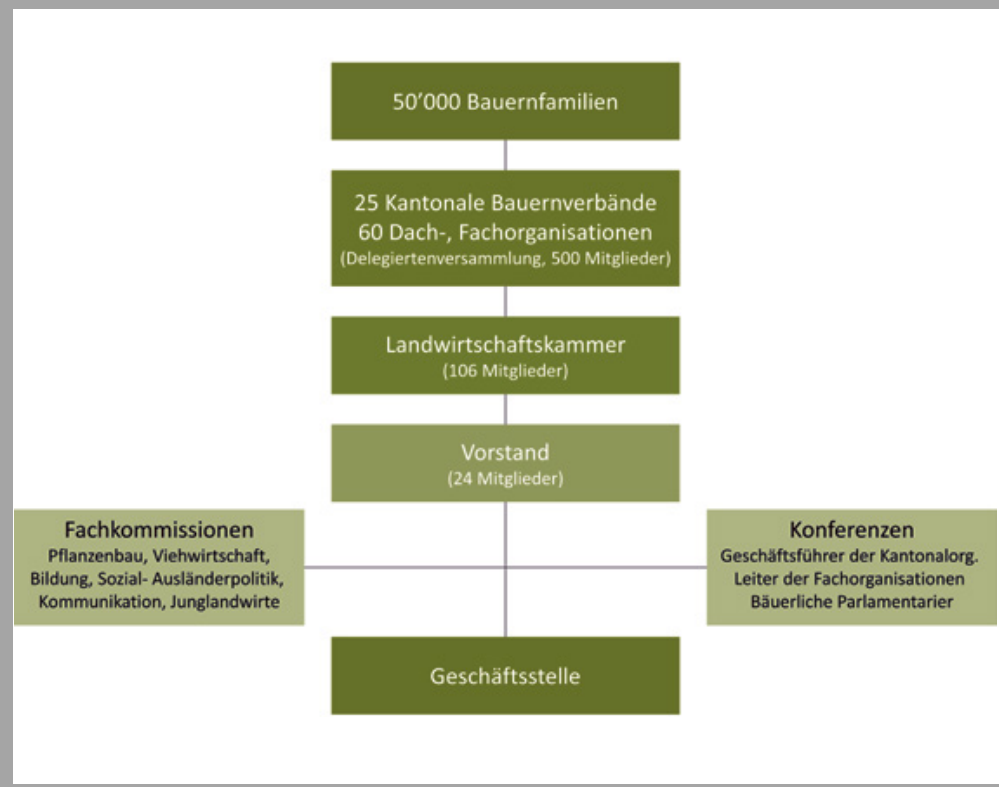

Quelle: SBV

Der Schweizer Bauernverband tritt in der Schweizer Agrarpolitik als besonders gut organisierte und mächtige Interessengruppe auf und kann viele agrarpolitische Entscheidungen beeinflussen. Gegeben seiner Grösse und Heterogenität überrascht dieser grosse Einfluss auf den ersten Blick. Vieles lässt sich allerdings durch die gute Organisation des Verbandes erklären. Die Charakteristik des Verbands ist, dass er sowohl vertikal wie auch horizontal gegliedert ist. Die vertikale Gliederung mit ihrer hierarchischen Struktur und dem breiten föderalistischen Aufbau garantiert dabei die Integration breiter Teile der ruralen Bevölkerung. Die Bäuerinnen und Bauern sind nicht direkt Mitglied des SBV, sondern sie gehören einem kantonalen Verband oder einer lokalen Genossenschaft an, welche die eigentlichen Mitglieder des SBV sind. Das hat zur Folge, dass praktisch sämtliche Bäuerinnen und Bauern Mitglied des SBV sind. Die horizontale Gliederung in thematische Bereiche wie Soziales und Dienstleistungen oder Kommunikation und Services ermöglicht eine effiziente Organisation und die Bereitstellung der oben erwähnten, breitgefächerten selektiven Anreize wie Versicherungen oder Beratungsleistungen. 
Durch diese gute Organisation ist der Schweizer Bauernverband in der Lage, innert kurzer Zeit eine Initiative auf die Beine zu stellen und sie ermächtigt ihn so zu einer Art Vetoposition in der Schweizer Agrarpolitik. Er übernimmt dadurch eine zentrale Rolle im Agenda-Setting der Agrarpolitik. Ein wichtiger Aspekt dabei ist, dass die Landwirtschaft schon lange von staatlicher Unterstützung profitiert. Sie wäre also ein potenzieller Verlierer von Deregulierung und Liberalisierung. Die Verluste sind dabei viel direkter als mögliche Gewinne bei den Konsumentinnen und Konsumenten. Gleichzeitig haben die Bäuerinnen und Bauern durch ihre breite Abstützung im ländlichen Raum viele Vertreter direkt im Parlament. Bei den Wahlen 2019 gelangten insgesamt 32 Vertreterinnen und Vertreter der Landwirtschaft ins Parlament. Das entspricht mehr als $12 \%$ und liegt daher deutlich über dem Anteil der Bäuerinnen und Bauern in der Bevölkerung. Ein wichtiger Grund dafür ist auch, dass der SBV schon seit 1897 existiert und seit Anbeginn unterschiedliche politische Parteien vereint.

Mit anderen Worten, eine Organisation, die heterogene Interessen einer grossen Gruppe vertritt, wie beispielsweise der Schweizerische Bauernverband (SBV), braucht Anreize, von denen die vertretenen Bäuerinnen und Bauern auch privat profitieren können. Der Schweizer Bauernverband beispielsweise bietet verschiedene private Dienstleitungen, wie Versicherungen, Beratung und Dienstleistungen, an. Die Beteiligten profitieren einerseits privatwirtschaftlich von ihrer Mitgliedschaft. Andererseits erhöhen sie gleichzeitig das Gewicht des Bauernverbands in den politischen Verhandlungsprozessen (Box 15). Das öffentliche Gut, die Vertretung der Landwirtschaft, erfolgt als «Nebenprodukt» der Erbringung von privaten Leistungen (Olson, 1979). Auch Genossenschaften verfolgen oft als Grundziel privatwirtschaftliche Interessen und bieten als sekundäres Produkt eine politische Interessenvertretung ihrer Mitglieder an. Ein anderes Beispiel sind die Umweltschutzorganisationen, welche ebenfalls in vielen Fällen ein privates Gut offerieren, wie beispielsweise eine Zeitschrift oder andere Formen von Information.

Eine Ursache für die Bereitstellung selektiver Anreize durch private Güter, um eine Gruppe zu organisieren, die dann aber öffentliche Güter bereitstellt, liegt im Phänomen des Trittbrettfahrens. Dieses umschreibt den Anreiz des Individuums, von öffentlichen Leistungen zu profitieren, z.B. vom politischen Lobbying, ohne dass man selbst etwas dazu beiträgt, z.B. in Form von Mitgliederbeiträgen oder Abgaben. Die Ursache des Trittbrettfahrens liegt in der unterschiedlichen Bestimmung der Nachfrage von privaten und öffentlichen Gütern. Während für ein privates Gut eine horizontale Aggregation der individuellen marginalen Zahlungsbereitschaft erfolgt, muss diese für ein öffentliches Gut vertikal sein, weil bei öffentlichen Gütern keine 


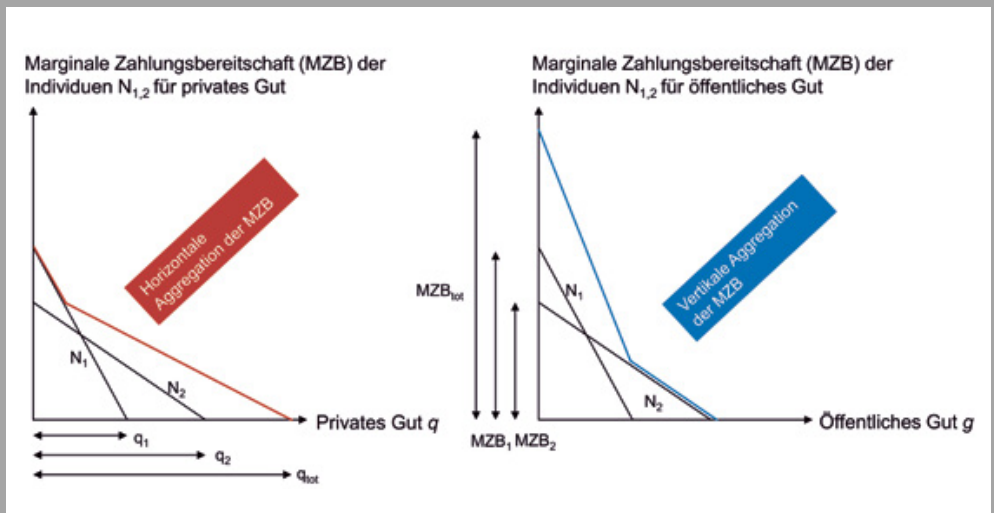

Die Nachfrage nach einem Gut ergibt sich aus der Summe der marginalen Zahlungsbereitschaften (MZB) der Konsumenten. Für ein privates Gut $q$ besteht eine Rivalität im Konsum. Das heisst, wenn beispielsweise der Konsument $\mathrm{N}_{1}$ einen Apfel isst, dann kann $\mathrm{N}_{2}$ den gleichen Apfel nicht mehr konsumieren. Mit anderen Worten: Da im Konsum des Gutes eine Rivalität besteht, können von $\mathrm{N}_{1}$ und $\mathrm{N}_{2}$ nicht die gleichen Einheiten konsumiert werden. Die totale nachgefragte Menge $q_{\text {tot }}$ ergibt sich daher aus der horizontalen Summe von $\mathrm{q}_{1}$ und $\mathrm{q}_{2}$. Die horizontale Aggregation folgt der Logik, dass aus einer höheren Anzahl an Konsumenten, die ein Produkt zu kaufen bereit sind, ein höherer Preis bei gegebener Menge resultiert. Die Nachfrage wird folglich über den Preis festgelegt. Für ein öffentliches Gut dagegen besteht keine Rivalität im Konsum. Wenn eine Menge des öffentlichen Gutes bereitgestellt wird, dann ist der Nutzen für die Individuen $\mathrm{N}_{1}$ und $\mathrm{N}_{2}$ derselbe. Die Aggregation der individuellen Zahlungsbereitschaften erfolgt daher vertikal und die Höhe der totalen Nachfrage wird über die Menge und nicht über den Preis festgelegt.

Rivalität im Konsum vorliegt. Die marginale Zahlungsbereitschaft ist also abhängig von der angebotenen Menge und nicht von deren Preis (Box 16). Als Konsequenz fehlt dem Nachfrager nach einem öffentlichen Gut das Preissignal: Er weiss nicht, ob seine Zahlungsbereitschaft tatsächlich zur Bereitstellung des öffentlichen Gutes führt. Das heisst, im Fall einer vertikalen Aggregation der marginalen Zahlungsbereitschaft sieht das einzelne Mitglied nicht, wie gross sein unter Umständen sehr geringer Beitrag überhaupt ist. Da er davon profitiert, wenn andere mit der gleichen Zahlungsbereitschaft für die öffentliche Leistung bezahlen, besteht ein Anreiz, sich nicht zu beteiligen und trotzdem von der öffentlichen Leistung zu profitieren. Im Fall des 
politischen Lobbyings lässt sich das Angebot nur schwer verkleinern. Deshalb dienen selektive Anreize dazu, mehr Mitglieder bzw. Konsumenten anzulocken. Durch ein günstiges Angebot werden Mitglieder angeworben und durch die vergrösserte Anzahl an Mitgliedern steht nun eine grössere Zahlungsbereitschaft für politische Aktivitäten zur Verfügung.

Es ist wichtig, die theoretischen Grundlagen für kollektives Handeln in der Agrarpolitik zu kennen, weil sie einen wichtigen Erklärungsansatz für den Einfluss der unterschiedlichen Akteure auf die Agrarpolitik leisten. Die politische und gesellschaftliche Stärke des Bauernverbands beispielsweise gründet nicht einfach auf einer starken parlamentarischen Vertretung. Sie folgt aus einem komplexen Prozess, welche subsidiäre Organisationen, Nebenprodukte und der politischen Vernetzung mit einschliesst.

\subsubsection{Bürokratie}

Neben den Interessenvertretern ist auch die Verwaltung (die Bürokratie) ein wichtiger Akteur in der Agrarpolitik, der Einfluss auf die resultierende Agrarpolitik nehmen kann. Dieser Einfluss ergibt sich daraus, dass Staatsangestellte andere Ziele verfolgen als ihre politischen Auftraggeber. Sie verhalten sich laut der Bürokratietheorie (Kirchgässner, 2010) nach analogen Zielen wie Angestellte aus der privaten Wirtschaft und streben nach einer Maximierung des Einkommens und einer Verbesserung der Arbeitsbedingungen. Sie können durch die Interpretation von Gesetzen und deren Umsetzung einen starken Einfluss auf die Wahl und Implementierung der agrarpolitischen Instrumente haben. Weil sie alleiniger Anbieter der geforderten Leistungen und damit keinem Wettbewerb ausgesetzt sind, bestehen für sie kaum Anreize, die Kosten der Leistungen zu senken. Im Gegenteil: Je grösser das Budget für die Agrarpolitik wird, desto grösser wird auch der Handlungsspielraum für die Administration. Man spricht in diesem Zusammenhang von der Budgetmaximierung. Sie widerspricht der Vorstellung, dass die Administration als eine Art «Staatsdiener» eine hierarchisch vorgegebene Funktion des Verwaltens ausübt. Diese Tendenz wird weiter durch die asymmetrische Information zwischen der Administration und den Politikern verstärkt. Oft haben die Experten in der Verwaltung mehr Wissen und Know-how über die Wirkung und Effizienz von agrarpolitischen Massnahmen als die Parlamentarierinnen und Parlamentarier. Ein wichtiger Pfeiler dieses Informationsvorsprungs in der Schweiz ist, dass das Bundesamt für Landwirtschaft (BLW) über die unterschiedlichen Gesetze und Verordnungen eine grosse Zahl von Daten und Informationen aus der Landwirtschaft zusammenführt und evaluieren lässt. In der Tendenz kann dies eine Ausdehnung der Leistungen der Verwaltung fördern, weil die Mitarbeiterinnen und Mitarbeiter in den Behörden diejenigen Massnahmen wählen, welche ihr Ansehen, ihren Einfluss und ihre Macht erhöhen können. Gleichzeitig gibt es in der Verwaltung strenge formale Richtlinien. Ohne gesetzliche Grundlage darf sie keine neuen Massnahmen oder Instrumente einführen. Innovative Elemente in 
der Agrarpolitik werden gemäss dieser Theorie von der Administration daher sehr zögerlich aufgenommen, weil vielfach erst eine neue Gesetzesgrundlage geschaffen werden müsste.

In den Überlegungen der neuen politischen Ökonomie geht es explizit nicht darum, die Verwaltung der Schweizer Agrarpolitik schlecht zu machen. Es ist aber wichtig zu erkennen, dass die Verwaltung aus theoretischer Sicht nicht einfach ein objektiver und wohlwollender Akteur in der Agrarpolitik ist und eigene Interessen verfolgt. Im Kontext der Schweizer Politik mit der Möglichkeit von Volksinitiativen und einer auf Konsens ausgerichteten Gesetzgebung mag dieser Aspekt weniger wichtig sein als in anderen Ländern. Nichtsdestotrotz bieten Aspekte der Bürokratietheorie wichtige Grundlagen für das Verständnis von agrarpolitischen Entwicklungen in der Schweiz.

\subsection{Der Politikzyklus}

Neben den grundlegenden Elementen der neuen politischen Ökonomie ist eine theoretische Einordnung des politischen Prozesses eine wichtige Grundlage für die Entstehung von Agrarpolitik. Eine grundlegende Basis für die Analyse von Strukturen und Prozessen in der Politik ist dabei der sogenannte Politikkreislauf (Politikzyklus). Dieser liefert eine theoretische Grundlage für die dynamische Entwicklung von Politikmassnahmen im Allgemeinen (Abbildung 14). Das Modell des Politikzyklus beschreibt grundsätzlich die unterschiedlichen und iterativen Phasen des Politikprozesses (Ingold et al., 2016). Grundsätzlich werden dabei fünf Phasen durchlaufen: die Problemdefinition, das Agenda-Setting, die Politikformulierung, die Implementierung und die Evaluation. In manchen Lehrbüchern wird die Problemterminierung als möglicher Ausstieg aus dem Politikzyklus definiert. Üblicherweise wird eine gewisse Problemstellung in der Agrarpolitik aber nicht im eigentlichen Sinne gelöst. Vielmehr verändern sich die Wahrnehmung und die Problemstellung so, dass der Zyklus kontinuierlich durchlaufen wird und lediglich die Problemstellung neu definiert wird.

Der Ausgangspunkt für eine neue Politikmassnahme entsteht meist durch das Auftreten einer neuen Problemstellung. Dabei ist es wichtig, festzuhalten, dass die politische Entscheidungssituation in der Realität von den bereits bestehenden Entscheidungen und den politischen Institutionen beeinflusst wird (Swinnen, 2018). Dabei ist es wiederum zentral, dass im Politikzyklus dadurch auch Machtpositionen und politischer Einfluss verschoben werden. Das heisst, früher getroffene politische Entscheidungen bringen ökonomische Konsequenzen mit sich, welche die Verteilung der Macht oder des Einflusses unter den Akteuren ändern kann (Rausser und Swinnen, 2011). Diese Verschiebung der Machtpositionen kann zu Anpassungen in den politischen Entscheidungen führen. Die Definition des Problems wird von den bereits existierenden politischen und gesellschaftlichen Akteuren geprägt (Pfadabhängigkeit). 
Die Priorisierung oder Strukturierung eines Problems in der Agrarpolitik, das sogenannte Agenda-Setting, ist daher stark von den etablierten Akteuren der Agrarpolitik beeinflusst. Der Einfluss auf das Agenda-Setting nimmt zu, je grösser die Ressourcen für eine Mobilisierung der Akteure sind. Massenmedien, soziale Netzwerke, aber auch die Mobilisierung der Bevölkerung über Initiativen haben in den letzten zehn Jahren neue Impulse in das Agenda-Setting der Agrarpolitik gebracht. Die Deutungshoheit über die Probleme der Landwirtschaft hat damit in der Entwicklung der Agrarpolitik noch mehr Einfluss gewonnen. Nur was als Problem wahrgenommen, definiert und eingebracht wird, kann überhaupt in den Prozess der Agrarpolitik gelangen. Neben der Mobilisierung der Akteure spielt dabei auch die Wahrnehmungskonjunktur eine wichtige Rolle, z.B. durch Medienberichte, Evaluationsberichte oder spezifische Ereignisse wie z.B. den Fund von Pestizidrückständen in Gewässern (Schaub et al., 2020).

Ist ein Problem auf der Agenda, werden daraufhin durch die politischen Akteure Schwerpunkte in einer neuen Agenda festgelegt und Handlungsanweisungen (Politikformulierungen) ausgearbeitet, die durch die Verwaltung umgesetzt werden sollen. Der Schlüssel zu einer effektiven Agrarpolitik ist dabei die Festlegung der Ziele. Wie aus der Geschichte der Agrarpolitik bekannt ist, haben sich die politischen Zieldimensionen und deren Gewichtung über die Zeit verändert. Je klarer die Zielformulierung in dieser Phase ist, desto grösser ist die Wahrscheinlichkeit, dass effektive Politikprogramme umgesetzt werden. Die Zielsetzung spielt sich typischerweise auf zwei Ebenen ab (Ingold et al., 2016). Einerseits werden auf einer abstrakten Ebene die generellen Ziele und die staatliche Auslegung der Politiklösung festgelegt. Andererseits werden auf einer konkreten Ebene die generellen Ziele in Handlungsprogramme übersetzt. Das Handlungsprogramm mündet in Gesetzen, die als Grundlage für die Umsetzung von konkreteren Zielen durch Massnahmen und Instrumente dienen. Die ersten drei Phasen des Politikzyklus lassen sich zwar konzeptionell trennen, in der Realität sind sie aber oft durch vielfältige Interaktionen zwischen politischen und gesellschaftlichen Akteuren miteinander verknüpft.

Die Politikimplementierung der formulierten Policy ist der Vollzug der im Handlungsprogramm festgelegten Instrumente und Massnahmen durch die Verwaltung. Dabei sind die Verwaltung und deren Ressourcen ausschlaggebend für die konkrete Umsetzung und den Vollzug der entsprechenden Massnahmen. In der Agrarpolitik sind die finanziellen Mittel im Verhältnis zu anderen Wirtschaftspolitiken, aber auch im Vergleich zu Umweltpolitiken (Wald, Landschaft etc.) gross. Im Anschluss an die Umsetzung erfolgt daher in den meisten Fällen eine Evaluation der Politikmassnahme. Dabei müssen unterschiedliche Stufen der Evaluation mithilfe eines Wirkungsmodells analysiert werden. Wie im Kapitel 6.2 ausgeführt, stehen dabei die Relevanz, Effizienz (Cost-Efficiency) und Effektivität (Cost-Benefit) im Vordergrund. 


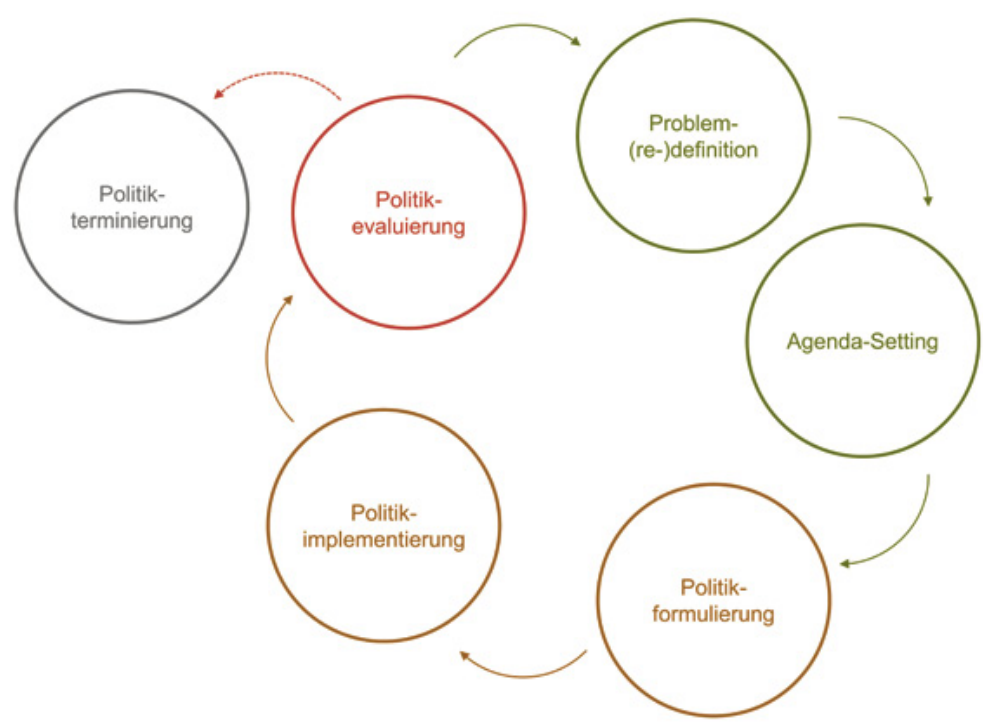

Abbildung 14. Elemente des Policy-Cycles nach Ingold et al. (2016): Problemdefinition (Notwendigkeit eines politischen Eingriffs), Agenda-Setting (Selektion, Prioritätensetzung und Strukturierung eines Problems abhängig von politischer Mobilisation [siehe Collective Action] und Wahrnehmungskonjunktur), Politikformulierung (Festlegung von Handlungsprogrammen und Gesetzen), Politikimplementierung (Anwendung der Gesetze: Durchführung und Umsetzung von Politikmassnahmen) und Politikevaluierung (empirische Bewertung von Vollzug und Wirkung).

Fällt die Evaluation mehrheitlich positiv aus, könnte der Politikzyklus terminiert und die Politikmassnahme so belassen werden. Werden allerdings weitere Verbesserungsmöglichkeiten entdeckt, was dem Regelfall entspricht, beginnt der Zyklus erneut mit einer erweiterten Problemdefinition. Der Politikzyklus ist geeignet, um konzeptionelle Diskussionen und Begriffe einordnen zu können, und damit geeignet, Politikprozesse aus einer allgemeingültigen Perspektive zu diskutieren. In der Realität hängen die Prozesse aber natürlich stark von den institutionellen Voraussetzungen ab.

\subsection{Prozesse der Schweizer Agrarpolitik}

\subsubsection{Rechtssetzungsprozesse in der Schweizer Agrarpolitik}

Weil in der Schweiz das Budget für die Bundesausgaben für den landwirtschaftlichen Sektor alle vier Jahre neu bestimmt wird, ist der politische Prozess ein fester Bestandteil der rationalen Agrarpolitik der Schweiz. Die Anpassungen werden jeweils in einem Paket als Agrarreform, z.B. AP14-17, AP22+, zusammengefasst. Massgebliche Veränderungen werden dabei vor allem dann bewirkt, wenn sich mit einer Verän- 
derung der Artikel 104 oder 104a in der Schweizer Bundesverfassung das übergeordnete politische Ziel der Politikmassnahme verändert. Änderungen der Verfassungsgrundlagen erfolgen zwingend über eine Volksabstimmung. Diese können als Referendum aus dem ordentlichen parlamentarischen Prozess hervorgehen oder aus Initiativen bzw. den daraus hervorgehenden direkten und indirekten Gegenvorschlägen (siehe Abbildung 16).

Neben der Verabschiedung des Budgets für die Bundesausgaben gibt es noch weitere Auslöser für die Anpassung von Gesetzen und Verordnungen. Dazu gehören in erster Linie parlamentarische Vorstösse wie Postulate und Motionen, welche vom Bundesrat explizit die Prüfung (Postulat) oder direkt die Anpassung einer rechtlichen Grundlage (Motion) verlangen. In einer parlamentarischen Initiative können die Parlamentarier einen eigenen Vorschlag zur Gesetzesänderung einbringen. Bundesrat und Verwaltung können aber auch aus eigenem Antrieb eine Anpassung vorschlagen, z.B. weil sie sich aus den Erfahrungen mit den bestehenden Rahmenbedingungen ableiten oder weil sie von aussen aufgrund internationaler Veränderungen aufgedrängt werden. Schliesslich können auch die Kantone über eine Standesinitiative einen Entwurf für Gesetzesanpassungen ausarbeiten lassen.

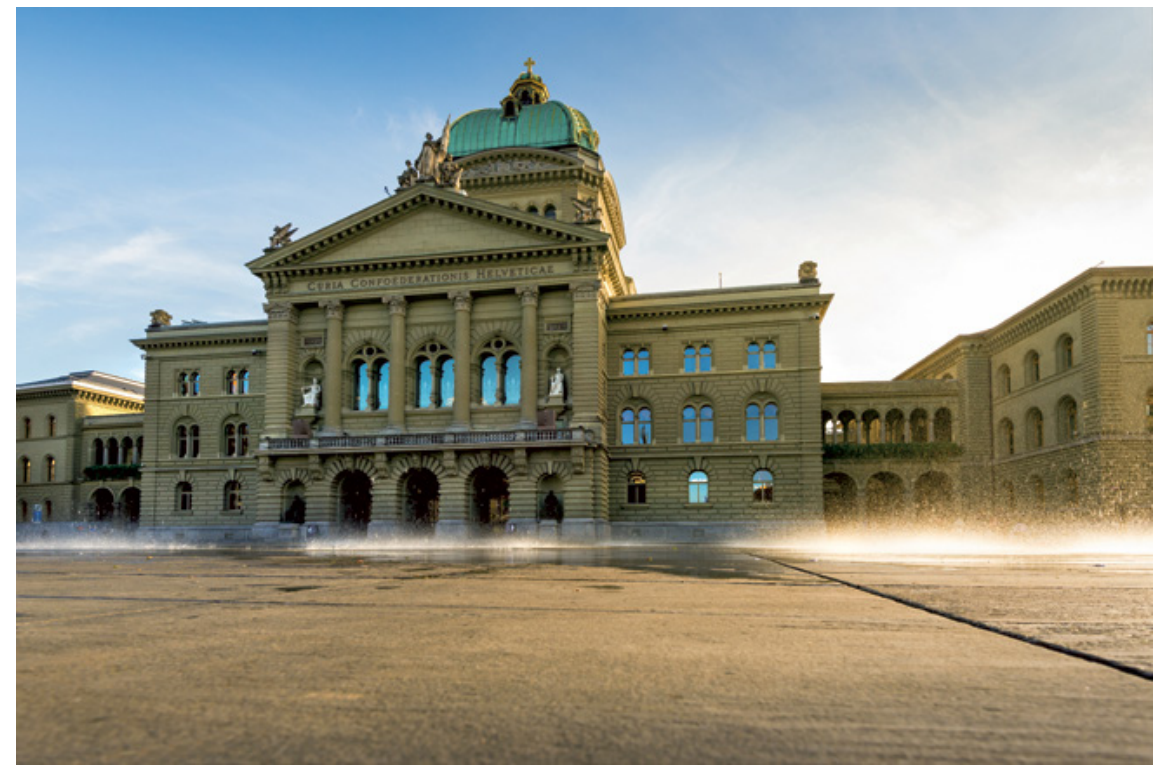

Abbildung 15. Die Veränderungen der Schweizer Agrarpolitik in den letzten 30 Jahren waren stark von der Verwaltung und dem Bundesrat geprägt. (Foto: Markus Thoenen/iStock) 
Das Verfahren zur Änderung von Gesetzen und Verordnungen erfolgt grundsätzlich in den folgenden drei Schritten: 1) Gesetzesentwurf unter der Führung des Bundesrats; 2) Verabschiedung des Gesetzes im Parlament und 3) Umsetzung und Inkrafttreten - wiederum auf der Ebene der Exekutive.

In der ersten Phase erarbeitet die zuständige Verwaltungseinheit, d.h. das Bundesamt für Landwirtschaft, einen Vorschlag für eine Gesetzesänderung (Vorentwurf). Dieser Vorschlag muss nicht nur die vorgeschlagene Änderung konkretisieren, sondern diese auch ausführlich begründen und deren Auswirkungen abschätzen. Dazu dienen oft agrarpolitische Evaluationen oder ex ante Modellberechnungen von Gesetzesänderungen durch die Agroscope. Dieser Vorschlag wird einer sogenannten Ämterkonsultation unterzogen, damit unterschiedliche Interessen bereits früh im Prozess berücksichtigt werden können. Für das Landwirtschaftsgesetz werden insbesondere dem Bundesamt für Umwelt, der eidgenössischen Finanzverwaltung, dem Staatssekretariat für Wirtschaft und der Bundeskanzlei die Möglichkeit gegeben, Stellung zum Vorschlag zu nehmen. Sämtliche Vorlagen, die nicht nur unbedeutende Auswirkungen haben, gehen anschliessend in eine Vernehmlassung. Das heisst, die Änderungen werden in den Vernehmlassungsunterlagen der Öffentlichkeit zur Stellungnahme unterbreitet. Alle interessierten Personen und Organisationen können ihre Bedenken und Verbesserungsvorschläge einreichen. Dazu gehören in erster Linie die Bauernverbände, die Wirtschaftsverbände, die Umweltschutzverbände, die Kantone und die Parteien. Das Bundesamt für Landwirtschaft sammelt die Vorschläge und Bedenken, veröffentlicht diese in einem Bericht und versucht diese nach Möglichkeit in einer Überarbeitung des Vorentwurfs zu berücksichtigen. Oft sind die Einwände und Vorschläge von den unterschiedlichen Akteuren jedoch widersprüchlich und nicht alle Anliegen können beachtet werden. Auf der Basis des überarbeiteten Vorschlags überweist der Bundesrat dem Parlament eine sogenannte Botschaft mit denjenigen Vorschlägen, die er nach der Ämterkonsultation und der Vernehmlassung als sinnvoll und mehrheitsfähig erachtet.

Im zweiten Schritt wird diese Botschaft in den parlamentarischen Kommissionen des Stände- und Nationalrats vorberaten, üblicherweise in der Kommission für Wirtschaft und Abgaben. Die Kommissionen stellen danach ihren Räten einen Antrag, welcher in den jeweiligen Kammern debattiert wird. Das Parlament kann den Vorschlag zur Gesetzesänderung des Bundesrates anpassen, zusätzliche Änderungen einfügen oder die ganze Vorlage ablehnen. Ein Gesetz kann in Kraft treten, wenn beide Räte zugestimmt haben. 


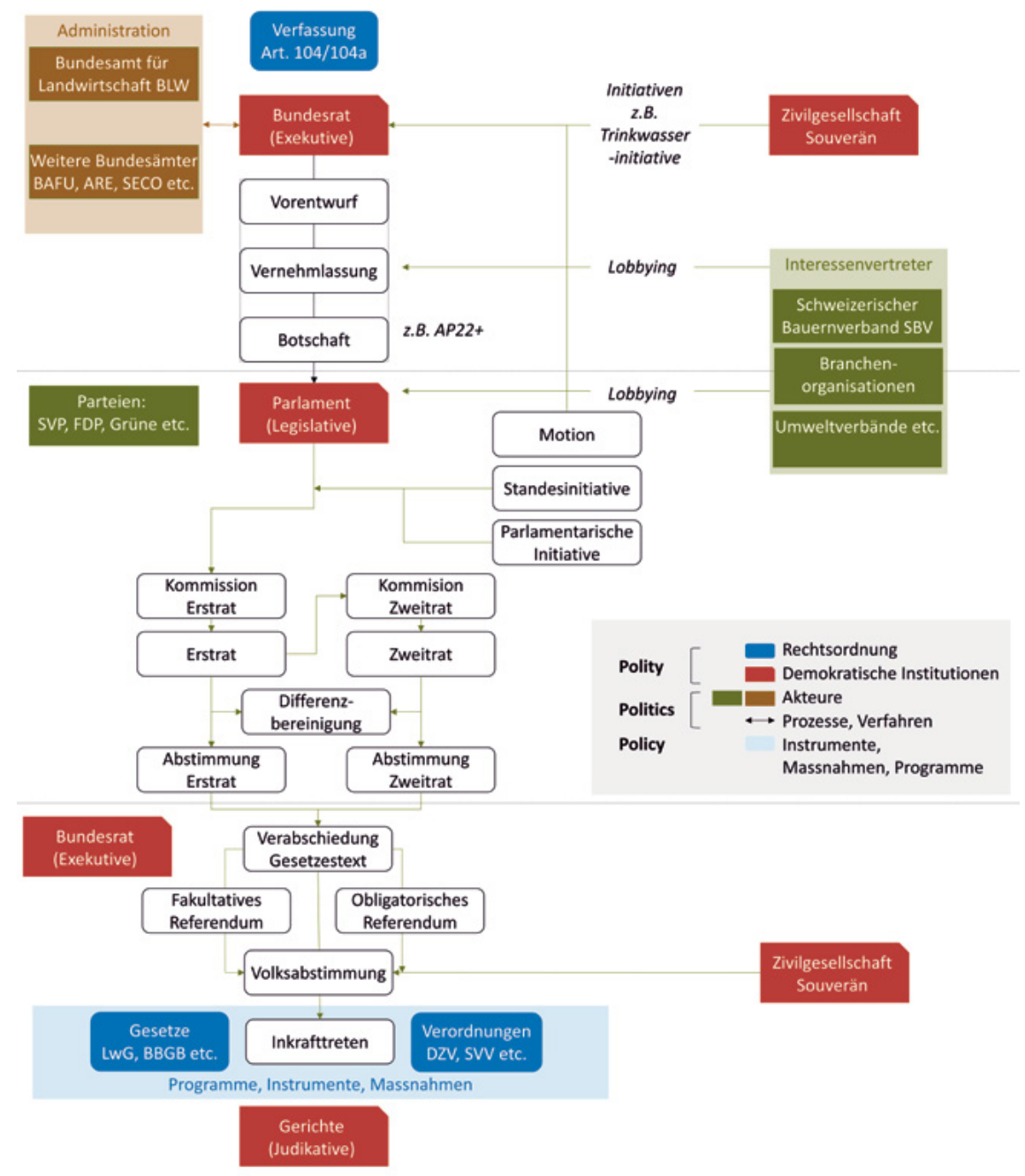

Abbildung 16. Polity, Politics, Policy im Kontext der Schweizer Agrarpolitik. Die Rechtsordnung besteht aus den Programmen, Massnahmen und den Instrumenten in der Agrarpolitik. Diese werden durch die demokratischen Institutionen, Legislative, Exekutive und Judikative, festgelegt. Prozesse und Verfahren in der Agrarpolitik involvieren in der Festlegung der Rechtsordnung neben der Administration und den politischen Parteien auch viele nicht staatliche Akteure wie Bauernverbände, Branchenorganisationen und Umweltverbände. Die formale Darstellung des Gesetzgebungsverfahren stammt von www.parlament.ch. 
Der dritte Schritt, wieder unter der Leitung der Exekutive, folgt mit der Verabschiedung des Gesetzes durch das Parlament. Wir ein Referendum gegen das Gesetz ergriffen, muss der Souverän darüber abstimmen. Ansonsten treten mit dem Gesetz auch die entsprechenden Verordnungen in Kraft. Diese werden parallel zur Gesetzesänderung durch das Bundesamt für Landwirtschaft entworfen. Diese können je nach Wichtigkeit auf Stufe Amt, Departement oder Bundesrat verabschiedet werden. Nach der Verabschiedung und der Publikation des Beschlusses tritt das Gesetz in Kraft. Ist das Gesetz einmal in Kraft, kann es im Rahmen von gerichtlichen Verfahren allenfalls zu Konkretisierungen oder Anpassungen kommen.

Grundsätzlich ist festzuhalten, dass der Rechtssetzungsprozess Zeit benötigt. Im Schnitt dauert eine Gesetzesanpassung mehr als vier Jahre und meistens länger als durch die Budgetplanung vorgesehen. Das hohe Reformtempo zu Beginn des Jahrhunderts hat daher abgenommen. Aus den vergangenen Agrarreformetappen geht aber deutlich hervor, dass die Prozesse in erster Linie dann effizient waren, wenn es einen klaren politischen Auftrag gab, beispielsweise aufgrund von breit abgestützten Motionen (Metz et al., 2021).

Aus der Erfahrung mit den Agrarreformen zeigt sich auch, dass die Veränderung der Schweizer Agrarpolitik in den letzten 30 Jahren stark durch die Verwaltung und den Bundesrat beeinflusst war (Hirschi et al., 2013). Da die Agrarpolitik eine Bundespolitik ist, wird sie durch institutionalisierte Prozesse auf Bundesebene wie beispielsweise der Vernehmlassung geprägt. Das müsste aber nicht zwangsläufig so sein. Die Volksinitiativen und die Einflussnahme des Bauernverbands im Parlament haben in den letzten Jahren zu einer Verschiebung der Kräfte geführt und insbesondere das Agenda-Setting dem klassischen Politikzyklus entzogen.

\subsubsection{Das agrarpolitische Netzwerk in der Schweiz}

Die neue politische Ökonomie liefert verschiedene Konzepte für das Verständnis von agrarpolitischen Entwicklungen. In der Realität laufen jedoch verschiedene Prozesse oft gleichzeitig und über mehrere institutionelle Hierarchien ab. Eine integrativere Perspektive auf die Prozesse und Abläufe der Agrarpolitik liefert das Konzept der "Governance». Der Begriff Governance hat viele Bedeutungen und wird in vielen unterschiedlichen Politik- und Wirtschaftsbereichen verwendet (Ingold et al., 2016). Im Kern ist Governance ein Oberbegriff für Steuerungsansätze, die nicht auf einer hierarchischen Regelsteuerung beruhen, sondern die Zusammenwirkung von Akteuren, Instrumenten und Prozessen auf verschiedenen Ebenen beschreiben und erklären und somit nicht nur auf den Staat beschränkt sind. Dabei werden z.B. Regierung und Vertreter von Zielgruppen als gleichrangige Partner in einem interaktiven Prozess betrachtet. Durch partizipative Verfahren kann die Basis politischer Prozesse breit abgestützt und so die Akzeptanz von politischen Regelungen erhöht werden. Im Kontext 
der Governance spielt daher die Koordination von unterschiedlichen Akteuren zur Erreichung von politischen Zielen eine zentrale Rolle.

Aus den Ausführungen zur neuen politischen Ökonomie geht hervor, dass sich die Akteure gleichsam wie Individuen in einem Marktumfeld verhalten. Die neue politische Ökonomie kann dabei auch berücksichtigen, dass die Akteure nur über eine begrenzte Rationalität verfügen. Das bedeutet, dass die Akteure beispielsweise nie über sämtliche relevanten Informationen verfügen können. Im Gegensatz dazu wird mit dem Konzept der Governance der politische Prozess weniger als Austausch von Interessen und Leistungen mit begrenzten Ressourcen betrachtet, sondern vielmehr als Auseinandersetzung oder Kooperation von unterschiedlichen politischen Vorsätzen, welche stark vom Kontext und deren Wahrnehmung sowie Interpretation abhängen. Im Gegensatz zur Strömung der neuen politischen Ökonomie sieht das Konzept der Governance also weniger strategisches und kalkuliertes Verhalten als Treiber der Agrarpolitik als vielmehr das institutionalisierte Zusammentreffen von Akteuren mit unterschiedlichen «Überzeugungen» und Interessen (Ingold et al., 2016).

Für die Analyse der Koordination von Akteuren im Rahmen einer Gesetzesreform können sogenannte Netzwerkanalysen beigezogen werden. In einer Netzwerkanalyse können nun die involvierten Akteure der Schweizer Agrarpolitik gemäss ihren Standpunkten und ihrer politischen Macht bezüglich einzelner agrarpolitischen Fragen einander gegenübergestellt werden. Der Fokus in den nachfolgenden Ausführungen liegt nun auf dem Netzwerk aus staatlichen und privaten Akteuren, die gemeinsam die Koordination und Steuerung der Agrarpolitik innerhalb der komplexen institutionellen Strukturen der Schweizer Politik übernehmen.

Das Konzept der Netzwerkanalyse fokussiert dabei auf das dynamische Zusammenwirken von unterschiedlichen Akteuren über Institutionen hinweg (Ingold et al., 2016). Akteure werden dabei in unterschiedliche Typen eingeteilt. Dabei wird einerseits zwischen öffentlichen und privaten Akteuren unterschieden. Öffentliche oder auch staatliche Akteure befassen sich mit der Ausarbeitung und Umsetzung von Politik. Sie umfassen alle Akteure der Exekutive, Legislative und Judikative sowie die öffentliche Verwaltung. Die politischen Parteien, aus welchen sich die Vertreter der Exekutive, Legislative und Judikative normalerweise konstituieren, sind ebenfalls öffentliche Akteure. Demgegenüber stehen die privaten Akteure, d.h. diejenigen, die von der Politik betroffen sind. In den allermeisten Fällen werden diese durch Verbände, Organisationen oder Vereine vertreten (Interessenvertreter).

Andererseits wird zwischen individuellen und komplexen Akteuren unterschieden. Komplexe Akteure sind letzten Endes ein Zusammenschluss von Individuen durch Kooperation. Die Bündelung der Interessen und Ziele in einem komplexen Akteur kann unterschiedlich umfassend sein. Die Theorie des kollektiven Handelns (Kapitel 11.1) 
fasst die ökonomischen Bedingungen zusammen, die es für den Zusammenschluss von Interessen braucht. In der Realität entsteht dabei ein Gradient an möglichen Zusammenarbeitsformen. Kollektive Akteure kooperieren, ohne dass ihre individuellen Organisationsstrukturen verschmelzen. Beispiele aus der Agrarpolitik sind die Interessengemeinschaft Agrarstandort Schweiz (IGAS), die Schweizerische Vereinigung Industrie und Landwirtschaft (SVIL) oder die Agrarallianz (ein Zusammenschluss von Organisationen aus den Bereichen Konsum, Umwelt, Tierwohl und Landwirtschaft). Korporative Akteure hingegen legen ihre Ressourcen zusammen und es entsteht eine eigene Einheit. Im Kontext der Agrarpolitik wäre beispielsweise der Schweizerische Bauernverband ein korporativer Akteur, weil er über eine eigene Organisation und finanzielle Ressourcen verfügt. Der Kern der Netzwerk-Perspektive ist nun, dass die Schweizer Agrarpolitik nicht nur durch die öffentlichen Akteure (den Staat) geprägt ist, sondern aus einer komplexen Interaktion der verschiedenen Akteurstypen.

Um die Agrargesetzgebung zu beeinflussen, formieren die Akteure je nach Inhalt unterschiedliche Koalitionen (Swinnen, 2018). Es entstehen dadurch immer wieder neue Koalitionen zu agrarpolitischen Sachfragen, die über Parteigrenzen hinweggehen. Grundsätzlich geht man in den Politikwissenschaften davon aus, dass die einzelnen Akteure über grundlegende politische Präferenzen verfügen (Core Beliefs), welche die Grundlage für politische Koalitionen ermöglichen. Zu diesen Grundeinstellungen gehört z.B., ob ein Akteur eine liberale oder interventionistische Einstellung bevorzugt. Darüber hinaus lassen sich aber auch Präferenzen der Akteure für einzelne Politikmassnahmen oder Instrumente bestimmen, also beispielsweise, ob man für eine Ausdehnung der Beiträge für Biodiversitätsförderflächen ist oder nicht. Diese Koalitionen lassen sich nun in sogenannten politischen Netzwerken darstellen und analysieren (Hirschi et al., 2013).

Im Kontext der Schweizer Agrarpolitik lassen sich die Akteure aufgrund der politischen Einstellung in grundsätzlich zwei Lager einteilen. Einerseits gibt es private und öffentliche Akteure, welche den Status Quo und die Erhaltung einer produktiven Landwirtschaft als vorrangig betrachten (siehe Abbildung 17, Panel A). Andererseits gibt es Akteure, die ganz unterschiedliche Grundeinstellungen miteinander verbinden. Wichtig ist nun zu erkennen, dass diese grundlegenden politischen Präferenzen in Sachfragen neue Koalitionen bilden können (Abbildung 17, Panel B). Je nach Politikinstrument und deren Ausgestaltung kooperieren unterschiedliche Akteure miteinander. Daraus ergeben sich in vielen Fällen Kompromissentscheidungen, indem unterschiedliche agrarpolitische Instrumente gegeneinander «getauscht» werden. Eine politikwissenschaftliche der AP14/17 zeigte diesen Aushandlungsprozess in der Schweizer Agrarpolitik exemplarisch (Metz et al., 2021). So konnten sich in Bezug auf die inländische Stützung, z.B. Standardvertrag Milch oder Importsystem Fleisch, die konservativen Kräfte durchsetzen. Mit Blick auf die Umweltanliegen, LQB, BFF und 
andere Direktzahlungen, hatten jedoch die grünen und moderaten Interessenvertretungen klar mehr Erfolg.

Dabei spielt auch die Entscheidungsmacht oder die Reputation des jeweiligen Akteurs eine wichtige Rolle. Organisationen wie beispielsweise der Schweizerische Bauernverband sind mächtig, weil sie über eine grosse Vertretung im Parlament verfügen und weil sie aus eigener Kraft ein Referendum zu einer Gesetzesvorlage ergreifen können (Hirschi et al., 2013). Diese Referendumsmacht erhöht das Gewicht des Verbandes in der Suche nach Kompromisslösungen zwischen den einzelnen Akteuren. Kleinere Akteure verfügen dagegen nicht über die Ressourcen, um die vom Parlament verabschiedeten Gesetzestexte noch zu bekämpfen. Erst die Zunahme von Initiativen in den letzten Jahren (siehe Abbildung 17) hat dazu geführt, dass mittlerweile sogar private Individuen Gehör in den agrarpolitischen Debatten erhalten.

Grundsätzlich ist aber festzuhalten, dass die Entwicklung der Agrarpolitik stark von der Verwaltung geprägt ist. Die institutionalisierte Weiterentwicklung der Agrargesetzgebung durch die Budgetverhandlungen führt dazu, dass das Bundesamt für Landwirtschaft regelmässig Anpassungen in der Agrarpolitik vornimmt. In der Vernehmlassung werden anschliessend sämtliche privaten und öffentlichen Akteure konsultiert und meist nur noch konsensfähige Gesetzesänderungen in der Botschaft aufgenommen. Dies führt zu einer relativ hohen Pfadabhängigkeit der Agrarpolitik in der Schweiz. Wesentliche Änderungen der Agrarpolitik wurden in der Vergangenheit nur durch starken innenpolitischen Druck, beispielsweise aufgrund von Motionen im Fall der Direktzahlungen, oder äussere Schocks, wie im Fall der Zollreduktion durch die Gründung der WTO, ausgelöst.

Zurzeit befindet sich die Schweizer Agrarpolitik dann auch in einer Phase der Starre. Seit der letzten erfolgreichen Reform (AP14/17) und der Einführung des neuen Direktzahlungssystems hat sich in der Schweizer Agrarpolitik wenig verändert. Die Diskussion um die nächste Agrarreform (AP22+) wurde im Parlament durch den Druck bäuerlicher Vertreter immer wieder verzögert. Zuletzt hat der Ständerat verlangt, dass die gesamte Agrarreformetappe sistiert wird (Dezember 2020). Durch den Druck der anstehenden Initiativen wurden aber gleichwohl verschiedene Umweltaspekte in die Planung der Agrarpolitik aufgenommen. So führte eine parlamentarische Initiative zur Einführung von Absenkpfade für den Einsatz von Pflanzenschutzmitteln sowie den Stickstoffeinsatz in die Agrargesetzgebung. Nachdem die Trinkwasser- und Pestizidinitiative abgelehnt wurden, liegt die Umsetzung nun beim Parlament. Erste Massnahmen sollen im Jahr 2023 umgesetzt werden. 

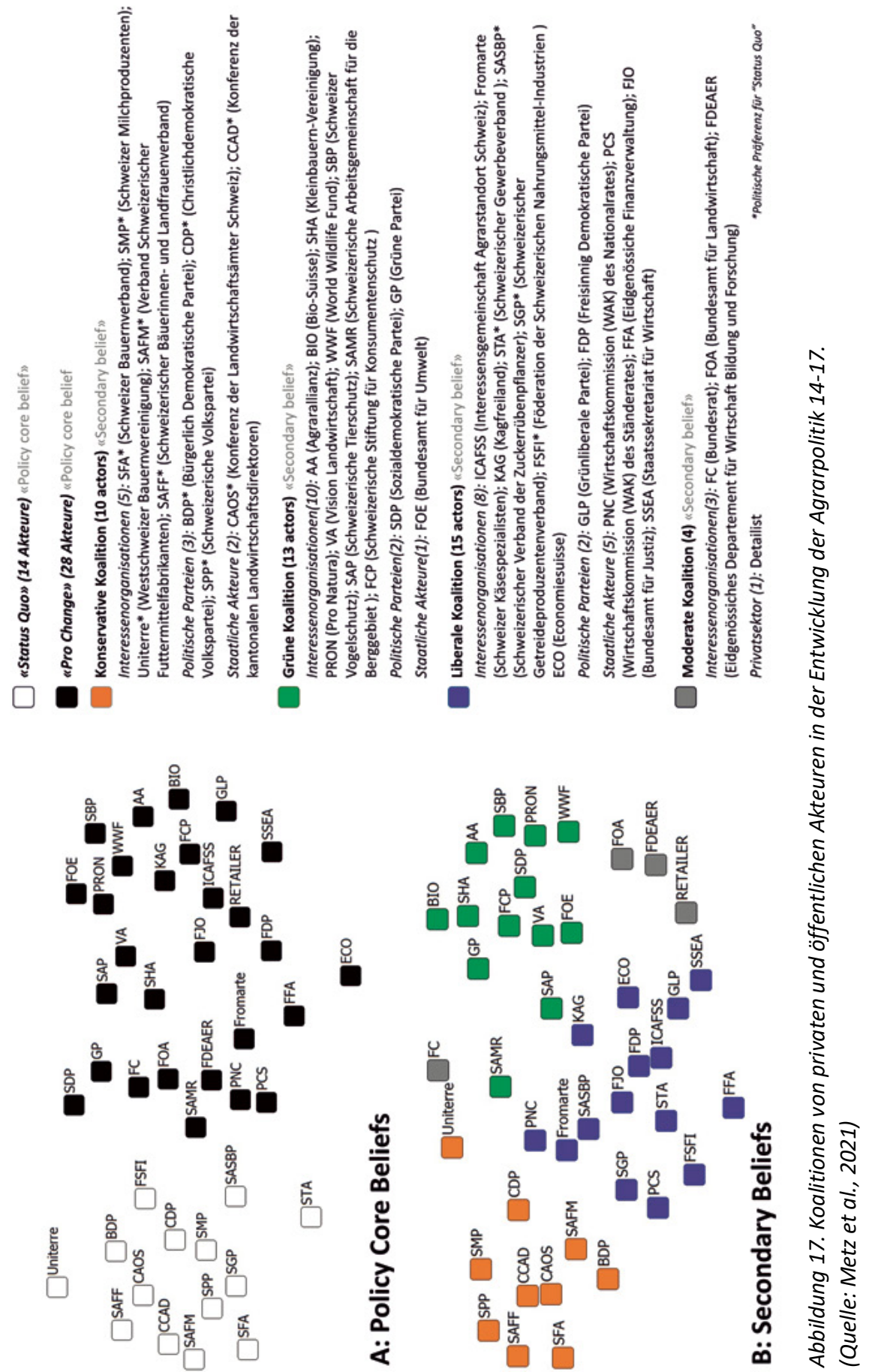


\section{ZUSAMMENFASSUNG}

Die Agrarpolitik wird fundamental durch die zugrunde liegenden politischen Prozesse definiert. Um diese politischen Prozesse zu erklären, greift die neue politische Ökonomie auf ökonomische Konzepte zurück: Politiker, Wähler, Interessenorganisationen und die Bürokratie streben nach der Maximierung ihres Nutzens, wodurch ein politischer Markt mit Angebot (Regelungen) und Nachfrage (Interessengruppen) entsteht. Dadurch lässt sich ein demokratischer Wettbewerb um Wählerstimmen und Abstimmungen als zentrale Triebkraft der Politiker ausmachen. Gleichzeitig nimmt die Interessenvertretung entscheidenden Einfluss auf die Politikumsetzung. Der Einfluss, den eine Interessengruppe dabei auf die Politik ausübt, ist stark abhängig von der Grösse dieser Gruppe. Kleine Gruppen mit homogenen Interessen sind dabei besser in der Lage, politische Einflussnahme auszuüben. Diese beiden Phänomene können viele in der Realität beobachteten Entwicklungen, wie beispielsweise den Protektionismus in der Landwirtschaft, gut erklären. Das Konzept der Governance und die Darstellung des Schweizer Agrarpolitiknetzwerkes erlaubt eine dynamische Betrachtung des Zusammenwirkens von vielen unterschiedlichen Akteuren in der Agrarpolitik. In der Schweiz ist die Verwaltung ein starker Akteur in der Entwicklung der Agrarpolitik. Sie wird massgeblich durch den Schweizer Bauernverband, der als Vetomacht fungiert, beeinflusst. Die Netzwerk-Perspektive lässt erkennen, dass Veränderungen der Instrumente oder Massnahmen in der Schweizer Agrarpolitik durch unterschiedliche Koalitionen entstehen. Das ermöglicht einen langsamen, aber stetigen Prozess der Anpassung.

\section{WEITERFÜHRENDE LITERATUR}

Die Grundlagen der politischen Ökonomie im Bereich der Agrarpolitik sind im Handbook of Agricultural Economics im Beitrag von Harry De Gorter und Johan Swinnen ausführlich beschrieben: "Political economy of agricultural policy».

Der Beitrag von Mancur Olson "Die Logik des kollektiven Handelns» ist zwar ein Text, der schon über 40 Jahre alt ist. Trotzdem ist die Argumentation auch heute noch von grosser Aktualität und Stringenz.

Das Buch von Amartya Sen Collective choice and social welfare bietet einen anspruchsvollen, aber erhellenden Blick in die Zusammenhänge zwischen sozialer Wohlfart, Demokratie und Ungleichheit.

Ein spannender Einblick in die politische Ökonomie stammt auch von Olivier de Schutter, ehemaliger Sonderberichterstatter für das Recht auf Nahrung, im Beitrag "The political economy of food systems reform» in der Zeitschrift European Review of Agricultural Economics. 
Einführung in die Schweizer Agrarpolitik

Der Artikel von Florence Metz, Eva Lieberherr, Aline Schmucki und Robert Huber mit dem Titel «Policy change through negotiated agreements: The case of greening Swiss agricultural policy» zeigt anhand der AP14/17 exemplarisch, wie Agrarreformen in der Schweiz erfolgreich umgesetzt werden können. 


\title{
12 Ausblick: Die Agrarpolitik in der Zukunft
}

\begin{abstract}
READERS' GUIDE
In diesem Buch wurde die Schweizer Agrarpolitik aus agrarökonomischer Perspektive betrachtet. Der Fokus lag auf der historischen Entwicklung, der bestehenden Ausgestaltung und den zugrunde liegenden agrarökonomischen Konzepten und politischen Prozessen. Im letzten Kapitel soll der Fokus auf der Zukunft der Schweizer Agrarpolitik liegen mit einem Zeithorizont von $20 \mathrm{Jah}$ ren. Dabei stehen drei Aspekte im Vordergrund: 1) die digitale Agrarpolitik; 2) der Klimawandel; und 3) die Ernährungspolitik. Das Lernziel ist, dass die Leserinnen und Leser einen geschärften Blick auf zukünftige Herausforderungen der Agrarpolitik entwickeln und diesen Blick mit dem Wissen über die bestehende Agrarpolitik verbinden können.
\end{abstract}

Es ist davon auszugehen, dass die grundlegenden Treiber, welche im dritten Kapitel dieses Buchs vorgestellt wurden, auch in 20 Jahren die Agrarpolitik noch prägen werden: Strukturwandel, Produktivitätsentwicklungen, Globalisierung und Handel, Nachfrageveränderungen und die Interaktion der Landwirtschaft mit der Umwelt. Die Agrarpolitik wird sich daher auch in Zukunft mit ähnlichen Problemstellungen konfrontiert sehen. Zielkonflikte, Preisvolatilität, Nahrungsmittelsicherheit und Ernährungssicherheit, Non-Point Source Pollution, Informationsasymmetrien, Marktmacht und die Integration von negativen und positiven Externalitäten in die Märkte für landwirtschaftliche Produkte wird die Akteure der Schweizer Agrarpolitik auch mittel- und langfristig beschäftigen. Zwei neue Einflussfaktoren werden aber mit Sicherheit die zukünftige Agrarpolitik mitprägen: die Digitalisierung und der Klimawandel. Diese beiden Treiber werden die Zielkonflikte und die Unsicherheiten in der Agrarpolitik erhöhen. Für die zukünftige Agrarpolitik wird es daher wichtig sein, die Herausforderungen der Zukunft ganzheitlich anzugehen.

\subsection{Digitale Agrarpolitik}

Die Digitalisierung der Wirtschaft macht auch vor der Landwirtschaft nicht halt. Neue Informations- und Kommunikationstechnologien in Kombination mit smarten Maschinen oder Robotern könnten bereits in naher Zukunft die Produktion von landwirtschaftlichen Rohstoffen fundamental verändern (Walter et al., 2017). Es besteht die berechtigte Hoffnung, dass diese Technologien zu einem Anstieg der Produktivität führen können, in einem Ausmass, wie es zuletzt in der Grünen Revolution gelang, 
jedoch ohne den Anstieg von Hilfsmitteln wie Dünger, Pflanzenschutzmittel oder Antibiotika und damit viel effizienter. Dies könnte einen wesentlichen Beitrag leisten zur Auflösung des Zielkonflikts zwischen hoher Produktivität und negativen Externalitäten der landwirtschaftlichen Produktion.

Eine solch fundamentale Veränderung der landwirtschaftlichen Produktionsprozesse würde auch die Art und Weise, wie der Landwirtschaftssektor geregelt würde, und den zugrunde liegenden politischen Prozess stark beeinflussen (Ehlers et al., 2021). Kurzfristig ist damit zu rechnen, dass innerhalb der bestehenden Agrarpolitik verschiedene Prozesse automatisiert werden. Dazu gehören beispielsweise die Kontrollen der Betriebe im Rahmen der Erbringung des ökologischen Leistungsausweises (mithilfe von Satelliten und Sensoren), die Führung der Tierverkehrsdatenbank (mithilfe von Apps) oder die Grenzkontrollen (mithilfe von Blockchains). Damit könnten auch die administrativen Kosten der Verwaltung und der Bäuerinnen und Bauern der Schweizer Agrarpolitik erheblich sinken.

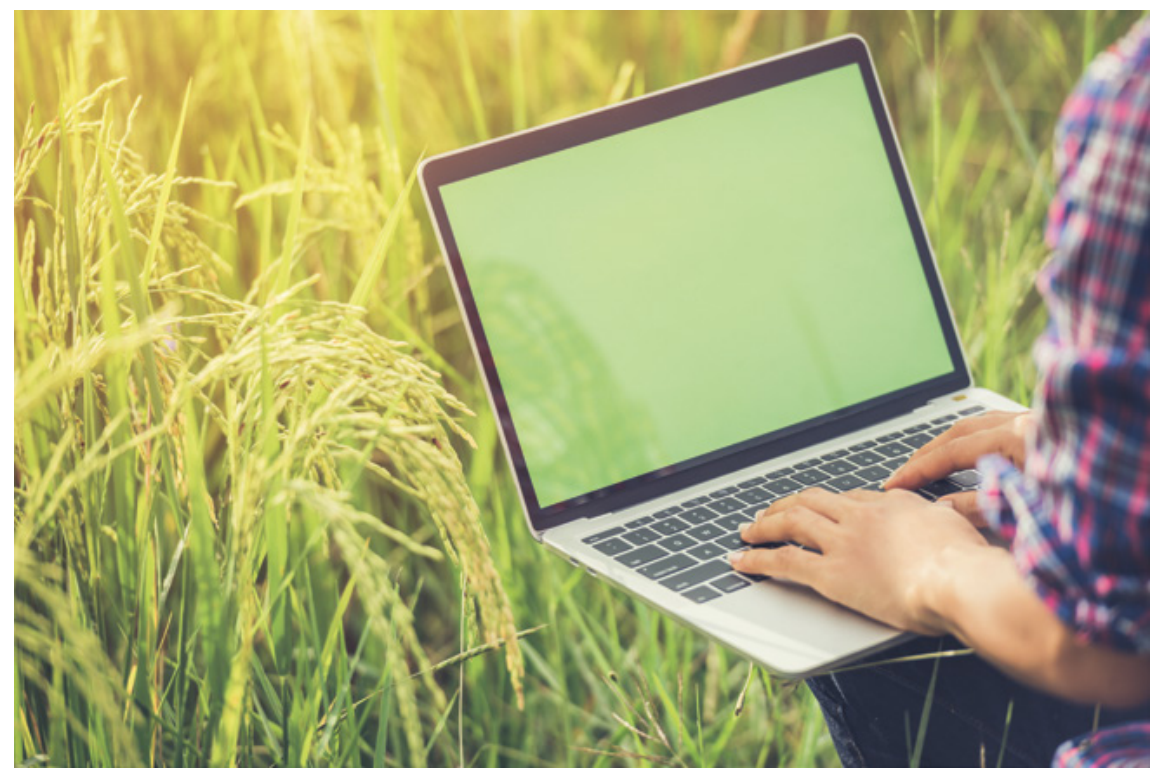

Abbildung 18. Die Digitalisierung der Landwirtschaft schafft auch neue Perspektiven für die Agrarpolitik. Die Integration von digitalen Technologien könnte es erlauben, das Targeting und Tailoring der agrarpolitischen Instrumente zu verbessern und gleichzeitig den administrativen Aufwand zu verringern. (Bild: iStock)

Eine digitale Agrarpolitik im weiteren Sinne kann aber langfristig noch sehr viel grössere Auswirkungen haben. Dabei stehen drei Aspekte im Vordergrund: 1) Neue Technologien, insbesondere Sensoren, können Auswirkungen der landwirtschaftlichen 
Produktion (Outcomes) messen, die zurzeit noch nicht oder nicht effizient genug beobachtet werden können. Ein Beispiel wäre die Messung von Stickstoffeinträgen oder Rückständen von Pflanzenschutzmitteln in Gewässern und deren Rückverfolgbarkeit zu einem bestimmten Landwirt. Damit könnte die Regelung von Non-Point Source Pollution in der Landwirtschaft angestrebt werden. Aber auch die Messung des Tierwohls oder von Biodiversität könnte in der Zukunft realisierbar werden. Wenn die Messung von Outcomes auch mit digitalen Technologien schwierig wäre, könnten andere Modelle, z.B. zu Bodeneigenschaften oder Landschaftsqualität, dazu beitragen, das Targeting von agrarpolitischen Massnahmen zu verbessern (Bartkowski et al., 2021). 2) Auf Basis dieser neuen räumlich und zeitlich hochaufgelösten Daten entstünden neue Ausgestaltungsmöglichkeiten für die agrarpolitischen Instrumente. Outcome-orientierte Massnahmen könnten viel effizienter und breiter eingesetzt werden, was von wissenschaftlicher Seite gefordert wird (Pe'er et al., 2020). Es könnte in Zukunft auch möglich werden, überbetriebliche Zielgrössen festzulegen und es so der Landwirtschaft erlauben, die kosteneffizientesten Massnahmen zur Zielerreichung umzusetzen. Auch das Tailoring der agrarpolitischen Massnahmen könnte zunehmen und damit Mitnahmeeffekte in der Unterstützung der Landwirtschaft reduzieren. Damit könnten mit dem gleichen Budget mehr Leistungen der Landwirtschaft zielgerichtet abgegolten werden. 3) Das Verhältnis zwischen den Akteuren entlang der Wertschöpfungskette können sich fundamental ändern. Damit könnten Regelungen im Bereich der Kennzeichnung, der Markttransparenz oder der Absatzförderung komplett neu konzipiert werden. Das könnte die Verhandlungsposition der Bäuerinnen und Bauern innerhalb der Wertschöpfungskette nachhaltig stärken und die Regelungsdichte im Bereich der landwirtschaftlichen Märke reduzieren, z.B. indem die Warenflüsse transparenter würden.

Schliesslich können Informations- und Kommunikationstechnologien auch die Problemdefinition und das Agenda-Setting der Agrarpolitik beeinflussen. Dazu gehören einerseits die sozialen Netzwerke, Medien und die im Internet verfügbaren Informationsressourcen, welche die Wahrnehmung der Landwirtschaft und deren Herausforderungen neu definieren können. War die Landwirtschaftspolitik ursprünglich eine Interessenpolitik, die mehrheitlich von den betroffenen Akteuren bestimmt wurde, ist sie in den letzten Jahren vermehrt der gesellschaftlichen Wahrnehmung und Kritik ausgesetzt (siehe Kapitel 2). Diese Entwicklung wird sich in der Zukunft fortsetzen und wohl an Bedeutung im Agenda-Setting der Agrarpolitik gewinnen. Digitale Datenauswertungen, z.B. über Machine Learning, könnten in diesem Zusammenhang den Akteuren der Agrarpolitik ganz neue Optionen aufzeigen. So könnte die Verwaltung beispielsweise zukünftige Herausforderungen mithilfe von GoogleTrend data frühzeitig erkennen und in den politischen Planungsprozess aufnehmen (Schaub et al., 2020). 
Der Grad, zu welchem die Agrarpolitik digital werden kann, hängt aber auch davon $a b$, in welchem Umfang die Bäuerinnen und Bauern die neuen Technologien anwenden. Zurzeit ist die Verwendung dieser Technologien in der Schweiz z.B. von Präzisionstechnologien im Pflanzenbau gering (Groher et al., 2020). Die Schweizer Agrarpolitik versucht in diesem Kontext mit Ressourcenprojekten die Adoption von neuen Technologien im Pflanzenschutz zu fördern. Im Projekt «Pflanzenschutzoptimierung mit Precision Farming» (PFLOPF) fördern der Bund und die Kantone Aargau, Thurgau und Zürich Technologien wie GPS-gesteuerte Pflanzenschutzgeräte oder sensorgesteuerte Unkrautregulierung im Acker- und Gemüsebau mit Beiträgen zwischen 50-200 Fr. pro Hektare und Jahr. Im Kontext der Anwendung von neuen Technologien stellt auch die Verwendung von neuen Züchtungsmethoden eine wichtige Herausforderung für zukünftige agrarpolitische Massnahmen dar. Die Einführung von Pflanzen auf Basis von CRISPR/CAS oder anderen Präzisionstechnologien in der Landwirtschaft basiert letztendlich auf einem politischen Entscheid. Zurzeit herrscht in der Schweiz ein Moratorium für solche Pflanzen (siehe Kapitel 7.4).

Im Kontext der Digitalisierung entstehen aber auch weitere Herausforderungen für die Agrarpolitik. Insbesondere der Frage nach der Datenhoheit kommt eine grosse Bedeutung zu. Ein "gläserner» Bauer, der von der Administration vollumfänglich kontrolliert werden könnte, ist in einem demokratischen und marktwirtschaftlich orientierten Land kaum vorstellbar (siehe dazu auch die Ziele der Agrarpolitik). Auch scheint klar, dass digitale Technologien zu einer Automatisierung der Wertschöpfungskette führen können und damit einer industriellen Landwirtschaft, in welcher der individuelle Landwirt keine Rolle mehr spielt, Vorschub leisten können. Es wird deshalb auch in Zukunft wichtig sein, institutionelle Netzwerke aufrechtzuerhalten, die marktwirtschaftliche und politische Interessen in einer effizienten Marktordnung zusammenbringen. Dafür wäre es für die Agrarpolitik wichtig, in die «digitalen» Kompetenzen aller Akteure entlang der Wertschöpfungskette zu investieren und notwendige Infrastruktur möglichst allen landwirtschaftlichen Akteuren zur Verfügung zu stellen. Dadurch können ungewollte soziale oder ökonomische Auswirkungen durch die Digitalisierung in der Landwirtschaft und der Agrarpolitik proaktiv angegangen werden (Ehlers et al. 2022).

\subsection{Klimawandel}

Neben der Digitalisierung wird auch der Klimawandel für die zukünftige Agrarpolitik eine wichtige Rolle spielen. Der Anstieg der Temperaturen und die Veränderungen der Niederschlagsmengen werden die Landwirtschaft vor neue Herausforderungen stellen. Der Koppelproduktion von landwirtschaftlichen Rohstoffen mit positiven und negativen Externalitäten kommt in diesem Kontext eine noch stärkere Bedeutung zu. Die Landwirtschaft ist nämlich nicht nur von der Klimaerwärmung betroffen, sondern trägt über den Ausstoss von Methan und anderen relevanten Klimagasen (Lachgas 
und Stickoxide aus der Düngung, $\mathrm{CO}_{2}$-Verluste durch Umbruch von Grünland) auch zur Klimaveränderung bei. Die Landwirtschaft muss sich daher nicht nur an veränderte Klimabedingungen anpassen (Climate Change Adaptation), sondern wird auch vor der Herausforderung stehen, einen Beitrag zur Reduktion von Treibhausgasemissionen zu leisten (Climate Change Mitigation).

Im Kontext der Anpassung steht für die Landwirtschaft vor allem die Zunahme von Extremereignissen wie Trockenheit, Hitzewellen, Starkniederschläge oder Frost im Vordergrund (Webber et al., 2020). Diese können eine direkte Wirkung auf wichtige Treiber der Agrarpolitik wie Preisvolatilität oder Umwelteffizienz haben. Für die Agrarpolitik stellt sich die Frage, ob die landwirtschaftlichen Betriebe auch in Zukunft eine betriebswirtschaftliche Lösung für den Umgang mit diesen Risiken finden werden. Treten Extremereignisse grossflächig und wiederkehrend auf, werden klassische Versicherungslösungen für die Landwirte schwerer zu finanzieren. Es stellt sich dann die Frage, ob es sich nicht um eine Art Marktversagen handelt, das durch staatliche Massnahmen geregelt werden sollte, beispielsweise über die Subventionierung von Versicherungen. Innovative Versicherungslösungen wie Index-Versicherungen könnten in diesem Kontext zu einem interessanten Instrument werden (Vroege et al., 2019). Für einen effizienten und effektiven Einsatz von staatlichen Versicherungslösungen in der Landwirtschaft ist aber die Ausgestaltung (Dimensionen) des Instruments zentral. Insbesondere das Zusammenwirken mit den Direktzahlungen, die ja bereits eine Art Versicherungseffekt haben können (siehe Decoupling im Kapitel 5.3), stellt die Ausgestaltung von subventionierten Versicherungslösungen vor Herausforderungen (Finger und Lehmann, 2012). Ausserdem besteht die Gefahr des Politikversagens, indem ineffiziente staatliche Massnahmen, mit hohem bürokratischem Aufwand, die privatwirtschaftlichen Lösungen aus dem Markt verdrängen.

Im Bereich der Senkung der Treibhausgasemissionen stehen in der Landwirtschaft in erster Linie technische Massnahmen im Vordergrund, z.B. eine Erhöhung der Stickstoffeffizienz im Pflanzenbau oder eine Anpassung der Fütterung in der Tierhaltung (Smith et al., 2008; Eory et al., 2018). Die Schweizer Agrarpolitik hat in diesem Kontext mit den Ressourceneffizienzbeiträgen, z.B. emissionsmindernde Ausbringverfahren, bereits einen ersten Schritt gemacht. Weitere Massnahmen werden auch im Rahmen von Ressourcenprojekten wie beispielsweise AgroCO $_{2}$ nept geprüft (Kreft et al., 2021). In diesem Projekt haben sich die Bäuerinnen und Bauern zum Ziel gesetzt, ihre Treibhausgasemissionen um 20 \% zu reduzieren und gleichzeitig die Wertschöpfung in ihren Betrieben zu erhöhen. Die Wirksamkeit dieser technischen Massnahmen in der Landwirtschaft sind aber begrenzt. Die technische Grundlage der Koppelproduktion lässt sich in einem Zeithorizont von 20 Jahren nicht grundsätzlich verändern. Die Gestaltungskraft der Agrarpolitik ist diesbezüglich beschränkt. Eine effektive Reduktion der Treibhausgasemissionen aus der Landwirtschaft wäre wohl nur durch eine Veränderung der Konsumgewohnheiten der Bevölkerung möglich (Muller et al., 2017b). 


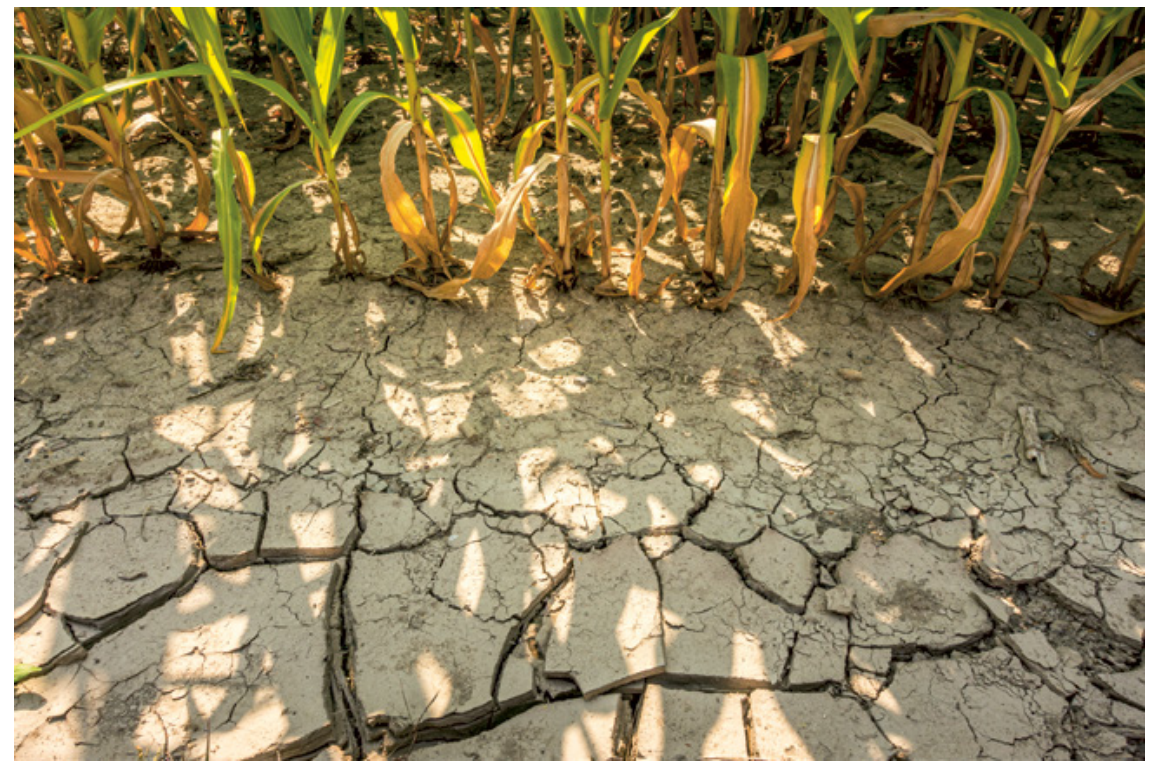

Abbildung 19. Der Klimawandel wird zu einer doppelten Herausforderung für die Landwirtschaft, weil sie nicht nur Betroffene, sondern auch Verursacherin der zukünftigen Erderwärmung ist. Die Agrarpolitik muss daher nicht nur die Rahmenbedingungen für die Adaption an den Klimawandel, sondern auch für die Reduktion der Treibhausgase in der Landwirtschaft schaffen. (Bild: no_limit_pictures/iStock)

\subsection{Von der Agrarpolitik zur Ernährungspolitik}

Die Digitalisierung und insbesondere der Klimawandel werden die Komplexität der Regelungen des Agrarsektors akzentuieren. Das kann zu einer Verschiebung der agrarpolitischen Ziele der Zukunft führen. Die Zielkonflikte werden dadurch aber nicht kleiner. Die Ausführungen in diesem Lehrbuch haben gezeigt, dass die Herausforderungen der Zukunft nicht alleine mit einer sektoralen Wirtschaftspolitik nachhaltig gelöst werden können, da die Bäuerinnen und Bauern sowie die vor- und nachgelagerten Industrien nicht die alleinigen Adressaten einer nachhaltigen Produktion von Nahrungsmitteln sein können (De Schutter et al., 2020).

Auch die Bürgerinnen und Bürger als Steuerzahler und Konsumenten müssen als Teil der Lösung betrachtet werden. Der Ansatz der Governance, der eine Integration des Akteurdreiecks Staat - Markt - Gesellschaft propagiert (Ingold et al., 2016), könnte die Grundlage für zukünftige Regelungen zwischen allen Akteuren des Ernährungssektors liefern. Damit diese Ansätze funktionieren können, braucht es eine stärkere Koordination verschiedener Politikfelder und eine partizipative Integration sämt- 
licher Akteure, um die Wissensbasis und das Know-how in der Agrarpolitik zu stärken und zu verbreitern (Metz et al., 2021). Eine Perspektive in diesem Sinne bietet auch das Konzept der sogenannten Food-Demokratie, welche zum Ziel hat, Reformwege für eine nachhaltigere Landwirtschaftspolitik zu entwickeln und umzusetzen (De Schutter, 2017).

Dazu bräuchte es einen Perspektivwechsel weg von der Betrachtung der Agrarpolitik als eine sektorale Wirtschaftspolitik hin zu einer integralen Ernährungspolitik basierend auf einer «Food-System»-Perspektive. Die Abbildung 20 fasst die Elemente eines solchen Land- und Ernährungssystems (Food-System) zusammen. Viele dieser Elemente wurden in diesem Buch im Kontext der Agrarpolitik ausführlich beschrieben wie beispielsweise die unterschiedlichen Treiber, die Wertschöpfungskette oder die politischen und institutionellen Prozesse. Wichtig ist hier zu erkennen, dass die einzelnen Elemente direkt oder indirekt miteinander verbunden sind und dass das Konsumentenverhalten und die Ernährungsweisen ebenfalls zentrale Elemente des Land- und Ernährungssektors sind. Damit wird illustriert, dass die Erreichung der grundlegenden Ziele einer integralen Ernährungspolitik - die Sicherstellung der Verfügbarkeit, des Zugangs und der Nutzbarmachung von Nahrungsmitteln für die Menschen - einen ganzheitlichen Blick benötigt.

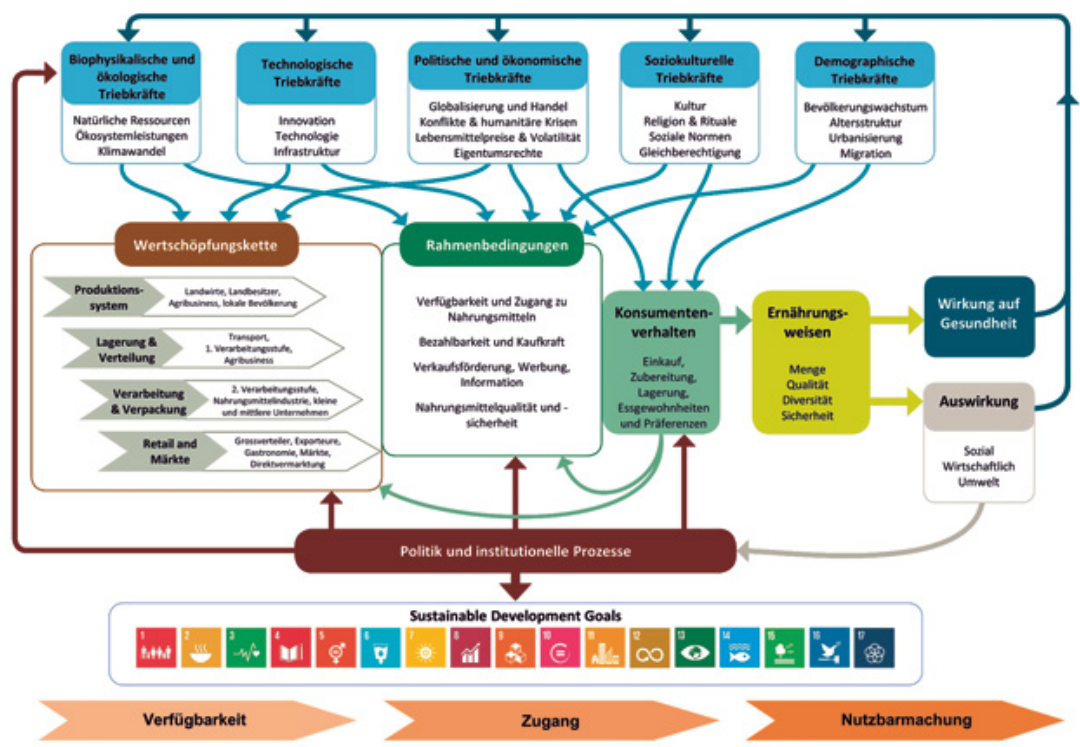

Abbildung 20. Elemente des Land- und Ernährungssystems. (Quelle: High Level Panel of Experts on Food Security and Nutrition (HLPE), FAO) 
Ein solcher ganzheitlicher Blick auf der Basis des Food-Systems könnte es erlauben, die Verantwortung der Gesellschaft zu stärken. Die Politik könnte stärker auf die tatsächlichen Auswirkungen (den Impacts) fokussieren und weniger auf die Mikrosteuerung von betrieblichen Aktivitäten/Leistungen durch agrarpolitische Massnahmen. Dadurch wäre es möglich, Ziele kohärenter miteinander zu verknüpfen und auf die tatsächlichen Bedürfnisse auszurichten (Gawith und Hodge, 2019). Neue Untersuchungen zeigen, dass solche integralen Package Deals auch von den Bürgerinnen und Bürgern besser akzeptiert würden (Fesenfeld et al., 2020). Das könnte eine Basis sein, um zukünftige Herausforderungen durch Freihandelsabkommen, Klimawandel und gesellschaftliche Ansprüche konsistenter zu regeln. Vielleicht wird es nie zu einem solchen Perspektivwechsel kommen, weil es die gesellschaftlichen, wirtschaftlichen und politischen Kräfte unserer Gesellschaft nicht erlauben. Es wäre aber trotzdem wünschenswert, wenn zukünftige agrarpolitische Reformen zumindest den Bogen weiter spannen und versuchen würden, die Herausforderungen der Zukunft integral anzugehen (Metz et al., 2021).

In diesem Kontext muss schliesslich auch der Begriff der Resilienz an Bedeutung in der Ernährungspolitik gewinnen. Mit zunehmender Komplexität und Unsicherheit kann es nicht das Ziel sein, die Landwirtschaft bis ins letzte Detail zu regeln. Erst Diversität entlang der gesamten Wertschöpfungskette erlaubt es, ein robustes, anpassungsfähiges und transformierbares, d.h. resilientes Ernährungssystem zu schaffen (Meuwissen et al., 2019). Letztendlich müssen in diesem Kontext auch die Bäuerin und der Bauer wieder im Fokus stehen. Für eine resiliente Landwirtschaft braucht es nicht nur eine Diversität der Ökosysteme, sondern auch eine Diversität der Bäuerinnen und Bauern, welche versuchen, die natürlichen Produktionsgrundlagen nachhaltig zu nutzen und für uns alle essenzielle Nahrungsmittel zu produzieren (GrêtRegamey et al., 2019).

\section{ZUSAMMENFASSUNG}

Die Agrarpolitik steht vor grossen Herausforderungen. Die gesellschaftliche Nachfrage nach sozialen und ökologischen Leistungen der Landwirtschaft nimmt zu. Gleichzeitig wird die Produktion von Nahrungsmitteln auch in der Zukunft die prioritäre Funktion der Landwirtschaft bleiben. Dabei werden die Digitalisierung und die Anwendung von neuen Technologien es ermöglichen, die Agrarpolitik neu zu denken. Das ist wichtig, weil insbesondere der Klimawandel die Regelung des Agrarsektors vor neue Herausforderungen stellen wird. Ein möglicher Ansatz dafür wäre es, die Agrarpolitik als integrale Ernährungspolitik zu konzipieren, die ökonomische, ökologische und soziale Herausforderungen integral zu steuern versucht. Der Begriff der Resilienz sollte dabei eine wichtige Rolle spielen. Nur eine Landwirtschaft, die widerstandsfähig ist, sich anpassen und verändern kann, wird es schaffen, diesen Herausforderungen gerecht zu werden. 


\section{WEITERFÜHRENDE LITERATUR}

Ein wichtiger Text stammt von Olivier de Schutter: "A «Common Food Policy» for Europe: How governance reforms can spark a shift to healthy diets and sustainable food systems» aus der Zeitschrift Food Policy. Eine gute Übersicht zu den Herausforderungen einer nachhaltigen Agrarpolitik.

Das Buch von John M. Antle und Srabashi Ray mit dem Titel Sustainable Agricultural Development: An Economic Perspective enthält eine agrarökonomische Perspektive auf die zukünftigen Herausforderungen. 


\section{Schlussbemerkung}

Das Ziel dieses Buches ist es, den Leserinnen und Lesern das Basiswissen für eine kritische Einordnung und Diskussion der Schweizer Agrarpolitik zu vermitteln.

Dazu wurde zuerst der Begriff Agrarpolitik definiert und im Kontext internationaler Entwicklungen eingeordnet. Anschliessend wurden die theoretischen und konzeptionellen Grundlagen der Agrarpolitik beschrieben. Dabei ging es in einem ersten Schritt um Grundbegriffe für das Verständnis der Schweizer Agrarpolitik und anschliessend um die Darstellung von agrarpolitischen Instrumenten. Diese Grundlagen wurden anhand der Geschichte der Agrarpolitik und der Schweizer Agrargesetzgebung vertieft und diskutiert. Schliesslich wurden in einem letzten Schritt die Prozesse und Institutionen der Agrarpolitik aus einer konzeptionellen Sicht beschrieben und anhand der Schweizer Agrarpolitik illustriert.

Es ist wichtig, an dieser Stelle festzuhalten, dass dieses Buch nicht die Absicht verfolgt, eine ganzheitliche Beurteilung der Agrarpolitik, insbesondere der Schweizer Agrarpolitik, vorzunehmen. Das Ziel ist, dass die Leserinnen und Leser die grundlegenden Konzepte und Prozesse hinter der Agrarpolitik kennenlernen und verstehen. Wie im Kontext des Werturteilsproblems in der Agrarpolitik ausgeführt (Kapitel 2), gibt es keine ultimative Form der Politik, die eine nachhaltige Nutzung der natürlichen Ressourcen, Nahrungsmittel für die gesamte Weltbevölkerung und gleichzeitig die Erhaltung der ländlichen Räume garantiert. Dieses Buch ist deshalb lediglich ein Startpunkt. Es soll eine fundierte und differenzierte Vertiefung mit der Schweizer Agrarpolitik ermöglichen und eine kritische Auseinandersetzung anregen - in der Hoffnung, dass sich mehr engagierte Personen für die Entwicklung der zukünftigen Agrarpolitik interessieren und, wenn immer möglich, in deren Ausgestaltung engagieren. 


\section{Glossar}

ABSATZFÖRDERUNG

AGENDASETTING

AGRARÖKOLOGIE
Das Instrument der Absatzförderung hat zum Ziel, den Konsum von einheimischen Lebensmitteln mithilfe von Werbung und PR im Inund Ausland zu erhöhen. Die Absatzförderung gehört in der Schweizer Agrarpolitik zum Bereich «Rahmenbedingungen für Produktion und Absatz».

Eine Phase innerhalb des Politikzyklus. Zusammen mit der Problemwahrnehmung ist das Agenda-Setting eine Art Filter, durch welchen wichtige Probleme auf die politische Tagesordnung gelangen - oder eben nicht. Medienberichte, politische Mobilisierung oder Ereignisse wie Pandemien, Umweltkatastrophen oder Wirtschaftskrisen können das Agenda-Setting beeinflussen.

Der Begriff Agrarökologie kann verschiedene Bedeutungen haben. Im engeren, wissenschaftlichen Sinn werden in der Agrarökologie die ökologischen Prozesse und Zusammenhänge im Kontext der Agrarproduktion beschrieben. Darüber hinaus definiert der Begriff aber auch eine gesellschaftliche Bewegung, die eine Transformation des bestehenden industriellen Produktionssystems in ein agrarökologisches System anstrebt. Agrarökologie in diesem Kontext beschreibt einen ganzheitlichen auf ökologischen und sozialen Konzepten beruhenden Ansatz, der das Management und die Gestaltung eines nachhaltigen Landwirtschafts- und Ernährungssystems anstrebt. Im Kern geht es darum, agrarökologische Mechanismen (z.B. Einsatz von Antagonisten oder nährstofffixierende Pflanzen etc.) für die Produktion in den Vordergrund zu rücken, Produktionskreisläufe zu schliessen und erneuerbare Ressourcen einzusetzen. Gleichzeitig soll Diversität in den Produktionssystemen eine resilientere Entwicklung ermöglichen. Dazu gehört auch, dass der Familienbetrieb und deren integriertes lokales Wissen gefördert wird und kulturelle und traditionelle Formen der Produktion und Ernährung ins Zentrum rücken. Dies soll eine effiziente und zirkuläre Land- und Ernährungswirtschaft ermöglichen. Die FAO unterstützt agrarökologische Anliegen und hat Elemente definiert, die als Grundlage für das Verständnis von Agrarökologie verstanden werden können: Diversität fördern, partizipative und inklusive Entwicklung von agronomischem Knowhow, ökologische Synergien nutzen, Effizienz steigern durch weniger Ressourcenverbrauch, Recycling, Resilienz, Werte und Traditionen in der Landwirtschaft und der Ernährung erhalten, verantwortungsvolle Steuerung der Systeme, zirkuläre und solidarische Wirtschaft. 
AGRARPOLITIK

AGRARPOLITISCHES NETZWERK

AGRICULTURAL TREADMILL

AGRIDEA

AGROSCOPE
Grundsätzlich wird die Agrarpolitik als sektorale Wirtschaftspolitik definiert. Die Landwirtschaft ist aber durch die Nutzung der natürlichen Ressourcen mit verschiedenen anderen Politikbereichen wie der Umweltpolitik oder der Raumplanung verknüpft. Die Agrarpolitik bedarf daher eines interdisziplinären Blicks, der sich auf verschiedene räumliche, zeitliche und institutionelle Ebenen bezieht.

Das agrarpolitische Netzwerk beschreibt die Verbindung von staatlichen und privaten Akteuren, die gemeinsam die Koordination und Steuerung der Agrarpolitik innerhalb der institutionellen Strukturen der Politik übernehmen.

Agrarökonomisches Konzept zur Auswirkung von Produktivitätsfortschritten in der Landwirtschaft. Die einzelne Bäuerin bzw. der einzelne Bauer ist ein Mengenanpasser, d.h., sie/er kann im Markt nur die Produktionsmenge an den Preis, nicht aber den Preis selber beeinflussen. Wenn nun landwirtschaftliche Betriebe eine neue Technologie einsetzen und dadurch die Produktivität zunimmt, dann werden diese Landwirte ihre Produktion ausdehnen können. In der Folge sinken die Preise, insbesondere wenn die Produktionsausdehnung auf eine unelastische Nachfrage trifft. Versuchen die Bäuerinnen und Bauern durch eine weitere Ausdehnung der Mengen die sinkenden Preise zu kompensieren, führt dies in einen Teufelskreis von tieferen Preisen und Mengenausdehnungen. Diese sich verstärkende Wechselwirkung zwischen Produktivitätsfortschritt, Mengenausdehnung und Preisrückgang wird als «Agricultural Treadmill» bezeichnet.

Die Agridea ist die landwirtschaftliche Beratungszentrale auf Bundesebene. Sie sichert den Austausch von Wissen und Erfahrungen zwischen den Akteuren der Landwirtschaft. Das bedeutet, die Agridea hilft, Netzwerke aufzubauen durch die Rolle als Bindeglied zwischen Wissenschaft und Bauernhof. Dazu stehen Forschung, Verwaltung und Politik in Form von Bundesämtern, Verbänden und Forschungsinstitutionen gegenüber von Bildungszentren, Beratungsstellen und den Bauern mit ihren Unternehmen. Die Agridea entwickelt Grundlagen und Methoden, stellt Informationen und Software bereit, bildet BeraterInnen aus und unterstützt und begleitet die Umsetzung von Projekten.

Agroscope ist das Kompetenzzentrum für landwirtschaftliche Forschung und dem Bundesamt für Landwirtschaft angegliedert. Agroscope forscht im Bereich einer nachhaltigen Land- und Ernährungswirtschaft (z.B. Züchtung, Wirtschaftlichkeit, Biodiversität etc.). 
AOA

AUKTION

BELASTUNGSGRENZE

BGBB

BRANCHENORGANISATION
AoA ist die Abkürzung für "Agreement on Agriculture». Dieses Abkommen ist Bestandteil der Verträge, welche im Rahmen der Gründung der WTO 1995 abgeschlossen wurden. Das Abkommen besteht aus drei Säulen: Marktzugang, inländische Stützung und Exportsubventionen. Die WTO-Mitgliedsländer verpflichteten sich mit dem AoA, den Marktzugang für landwirtschaftliche Produkte zu verbessern (Abbau von Grenzschutz), die inländische Stützung zu reduzieren (weniger produktionsgebunden) und die Exportsubventionen abzuschaffen.

Eine Auktion ist ein Versteigerungsverfahren. Man unterscheidet zwischen einem Leistungswettbewerb und dem Preiswettbewerb. Im Leistungswettbewerb wird der Preis festgelegt und die Leistung wird vom Landwirt angeboten. Im Preiswettbewerb wird die zu erbringende Leistung festgelegt und der Landwirt offeriert den Preis. In der Landwirtschaft kommen Versteigerungen im Rahmen der Zollkontingentsverteilung vor. Aus einer rationalen Perspektive würden sich auch gewisse Umweltleistungen der Landwirtschaft für eine Versteigerung eignen. Dies ist jedoch abhängig davon, wie heterogen die Leistung bzw. die Erbringungskosten der Landwirte sind.

Begriff aus dem Bundesgesetz über das bäuerliche Bodenrecht (BGBB). Die Belastungsgrenze soll die Überschuldung der landwirtschaftlichen Betriebe verhindern. Die Belastungsgrenze legt fest, dass landwirtschaftliche Grundstücke und Gewerbe nur bis zu einer definierten Grenze mit Grundpfandrechten belastet werden dürfen. Im BGBB entspricht die Belastungsgrenze dem um 35 Prozent erhöhten landwirtschaftlichen Ertragswert.

Das Bundesgesetz über das bäuerliche Bodenrecht (BGBB) regelt den Erwerb und die Veräusserung von landwirtschaftlichem Boden. Der Zweck des Gesetzes ist es, das bäuerliche Grundeigentum zu fördern und bodenbewirtschaftende Familienbetriebe zu erhalten. Es enthält privatrechtliche Beschränkungen, das heisst die Übernahme von Objekten (z.B. landwirtschaftlichen Gewerben) innerhalb der Familie und öffentlich-rechtliche Beschränkungen (z.B. das Zerstückelungsverbot oder das Realteilungsverbot für ganze Gewerbe). Ausserdem setzt es Massnahmen zur Verhütung der Überschuldung von landwirtschaftlichen Betrieben fest.

Eine Branchenorganisation ist für die Qualitäts- und Absatzförderung der Produkte einer gewissen Branche verantwortlich und verfolgt den Erhalt und die Förderung der Wertschöpfung sowie der Marktanteile in den in- und ausländischen Märkten (Selbsthilfemassnahmen). Eine solche Organisation setzt sich aus verschiedenen Mit- 

KRATIETHEORIE

COLLECTIVE ACTION THEORY

COMMAND AND CONTROL

CROSS-COMPLIANCE
BÜRO- $\quad$ Begriff aus der neuen politischen Ökonomie. Die Grundannahme der

gliedern der Branche zusammen. Diese Mitglieder können Produzenten bzw. Produzentenorganisationen, Verarbeiter sowie auch Einzelfirmen aus der Branche sein. Beispiele für Branchenorganisationen: Proviande oder Branchenorganisation Milch. Bürokratietheorie ist, dass sich Staatsangestellte gleich verhalten wie Angestellte in der Privatwirtschaft und nach einer Maximierung des Nutzens streben (Einkommen, Ansehen oder verbesserte Arbeitsbedingungen). Da die staatliche Administration (die Bürokratie) alleiniger Anbieter von politischen Leistungen ist, hat sie den Anreiz, staatliche Regelungen tendenziell zu erweitern, um ihren Einfluss und die entsprechenden staatlichen Ressourcen (z.B. Budgets) auszubauen. Da kein Wettbewerb herrscht, bestehen kaum Anreize, die administrativen Kosten der Leistungen zu senken.

Begriff aus der neuen politischen Ökonomie. Sie erklärt, weshalb es einer Gesellschaft schwerfällt, öffentliche Güter in einem ausreichenden Mass anzubieten. Dabei spielt die Nicht-Ausschliessbarkeit von öffentlichen Gütern eine wichtige Rolle. Da niemand ausgeschlossen werden kann und der eigene Beitrag zum öffentlichen Gut oft sehr gering ist, besteht die Gefahr des Trittbrettfahrens (Dritte profitieren vom Gut ohne dass sie dazu beitragen). Entscheidend für die Durchsetzung von öffentlichen Interessen und damit deren Leistungen oder Produkte ist gemäss der Collective Action Theory der unterschiedliche Einfluss, den Interessengruppen auf das politische Basissystem ausüben. Für dieses spielt die unterschiedlichen Gruppengrössen und die Homogenität der Interessen der verschiedenen Gruppierungen eine zentrale Rolle.

Kategorie von Politikmassnahmen. Grundsätzlich lassen sich Politikinstrumente in drei Kategorien unterteilen: Command and Control, ökonomische Anreize und informationsbasierte Massnahmen.

Wie der Name schon impliziert, schaffen Command-and-ControlInstrumente keine Anreize, sondern setzen Standards oder verbieten gewisse Aktivitäten. In der Schweizer Agrarpolitik kommen diese Instrumente in erster Linie im Tier- und Gewässerschutz zur Anwendung.

Ökonomische Anreize können mit Mindeststandards (siehe Command and Control) verbunden werden. Diese Verbindung garantiert, dass gewisse Vorschriften von sämtlichen Politikadressaten eingehalten werden, bevor sie berechtigt sind, von Subventionen zu profitieren. In der Schweiz dient der ökologische Leistungsnachweis als Cross-Compliance-Massnahme für den Erhalt von Direktzahlungen. 
DEAD

WEIGHT

LOSSES

DECOUPLING

DIREKT-

ZAHLUNGEN

ERNÄH-

RUNGS-

SICHERHEIT

ERTRAGS-

WERT

EVALUATION
Werden Cross-Compliance Massnahmen verletzt, kann der Bund der Bäuerin oder dem Bauern die Direktzahlungen kürzen.

Begriff aus der Wohlfahrtsökonomie. Ausgehend von einem Marktgleichgewicht beschreibt der Wohlfahrtsverlust den Effizienzverlust von staatlichen Eingriffen. Als Netto-Wohlfahrtsverlust wird der Konsumenten- oder Produzentenrentenverlust bezeichnet, der nicht umverteilt oder dem Staat zugutekommt.

Begriff aus der rationalen Agrarpolitik. Decoupling beschreibt die Trennung von Preis- und Einkommenspolitik im Agrarsektor. Historisch wurden die Einkommen der Bäuerinnen und Bauern über die Beeinflussung von Preisen sichergestellt. Das führte zu Überproduktion und Umweltproblemen. Ende der 1990er-Jahre wurde deshalb in der Europäischen Union und der Schweiz eine Anpassung der Politik vorgenommen: Die Stützung der landwirtschaftlichen Einkommen wurde durch Direktzahlungen sichergestellt und die Einkommenssicherung damit von den Marktpreisen entkoppelt (Decoupling). Dadurch wurden Veränderungen in Angebot und Nachfrage wieder in die Preisbildung auf Agrarmärkten integriert und Effizienzverluste reduziert.

Die Direktzahlungen sind ein agrarpolitisches Instrumentarium, um gemeinwirtschaftliche Leistungen des landwirtschaftlichen Sektors abzugelten wie z.B. die Versorgungssicherheit, die Pflege der Kulturlandschaft oder die Erhaltung der Biodiversität. Sie gehören zur Kategorie der «inländischen Stützung» und sind ein zentrales Steuerungsinstrument der Schweizer Agrarpolitik.

Ernährungssicherheit (Food Security) bedeutet, dass die Bevölkerung jederzeit Zugang zu einer ausreichenden Menge von hochwertigen und bezahlbaren Lebensmitteln hat.

Der Ertragswert entspricht dem Kapital, das mit dem Ertrag eines landwirtschaftlichen Gewerbes oder Grundstücks zu einem durchschnittlichen Zinssatz verzinst werden kann. Demgegenüber steht der Verkehrswert, welcher dem eigentlichen (Verkaufs-)Wert landwirtschaftlicher Betriebe entspricht. Der Ertragswert wird bei der Übergabe des landwirtschaftlichen Betriebs innerhalb der Familie angewendet. Das primäre Ziel dieser Regelung ist es, dass die Verschuldung der bäuerlichen Betriebe begrenzt wird.

Phase des Policy-Cycles (im engeren Sinn). Um die Wirkung von Politikmassnahmen auf ihre Effektivität und Effizienz zu prüfen, werden agrarpolitische Instrumente regelmässig evaluiert. Für die Evaluation wird ein Wirkungsmodell benötigt. 
EXPORT-
SUBVENTION

EXTERNALITÄT

FAO

GEFAN-

GENEN-

DILEMMA
Mit Exportsubventionen wird der Verkauf von inländischen Produkten ins Ausland verbilligt. Dadurch sinkt der Preis auf dem Weltmarkt und es entsteht immer ein Wohlfahrtsverlust. Die WTO führt daher Exportsubventionen als eigene Kategorie (neben Marktzugang und inländischem Support) und zwingt ihre Mitglieder, diese handelsverzerrenden Massnahmen abzuschaffen. Die Schweiz hat 2019 die letzte Exportsubvention im Landwirtschaftsbereich (das sogenannte «Schoggigesetz») durch eine privatrechtliche Regelung mit Beiträgen ersetzt.

Externe Effekte (Externalitäten) sind Auswirkungen von wirtschaftlichen Entscheidungen auf Dritte, für die niemand bezahlt oder kompensiert wird. Externalitäten in der Landwirtschaft können positiv oder negativ sein. Klassische Beispiele für negative Externalitäten sind Stickstoffauswaschungen in Gewässer oder Pestizidrückstände in Lebensmitteln. Positive Externalitäten sind beispielsweise die Pflege der Kulturlandschaft oder die dezentrale Besiedlung. Externe Effekte sind oft mit Marktversagen verbunden und daher ein wichtiger Grund für staatliches Eingreifen in die Landwirtschaft. Mit agrarpolitischen Massnahmen sollen die externen Effekte in die wirtschaftlichen Entscheide internalisiert werden.

Die Ernährungs- und Landwirtschaftsorganisation der Vereinten Nationen (Food and Agriculture Organization of the United Nations, FAO) setzt sich aus 194 Mitgliedstaaten zusammen. Die Ziele der FAO sind die Bekämpfung von Hunger und Fehlernährung und die Sicherstellung von Ernährungssicherheit weltweit. Die Organisation fungiert als Wissens- und Forschungsnetzwerk in den Bereichen Landwirtschaft, Forstwirtschaft und Fischerei. Sie ist damit ein wichtiger Akteur für die Beratung, den internationalen Austausch und die weltweite Koordination von Massnahmen zur Erhöhung der Produktivität und Verbesserung der Nachhaltigkeit im Ernährungsbereich. Die Schweiz ist seit 1946 Mitglied.

Das Gefangenendilemma ist eines der bekanntesten Konzepte aus der Spieltheorie. Es besagt, dass die Kooperation zwischen den Spielern den höchsten Nutzen bringen würde, sich aber für die einzelnen Spieler unter Umständen ein egoistisches Verhalten lohnen würde. Weil die Auswirkung der eigenen Entscheidung auch vom Verhalten des Mitspielers abhängt (Interdependenz), ergeben sich unterschiedliche strategische Optionen für die Lösung des Gefangenendilemmas. Im Kontext der Agrarpolitik lässt sich mit dem Gefangenendilemma beispielsweise die Entscheidung zur Wahl des Grenzschutzes erörtern. Ohne Kooperation werden protektionistische Massnahmen oft gegenüber dem Freihandel vorgezogen. 
GEMEINWOHL

GREEN BOX

GRÜNE REVOLUTION

HAND-

LUNGSPROGRAMM
Als Gemeinwohl wird die Aggregation des Wohlergehens jedes Individuums in einer Gruppe verstanden z.B. in einem Land oder einer Gemeinde. Die Idee ist, dass es eine soziale Wohlfahrtsfunktion gibt (eine Art Nachfragefunktion), die es erlaubt, den grössten Nutzen für die Gemeinschaft zu erzielen. Diese Idee wird von der Theorie der neuen politischen Ökonomie verworfen. Aus einer liberalen Position wird davon ausgegangen, dass ausschliesslich Individuen ihre eigenen Präferenzen kennen. Unter dieser Annahme lässt sich aus dem Arrow-Theorem ableiten, dass es in einer Demokratie nicht möglich ist, eine solche Gemeinwohlfunktion zu definieren. Die Folgerung daraus ist, dass es den politischen Akteuren auch unmöglich ist, das Gemeinwohl zu verfolgen bzw. zu optimieren und es zwingend politischen Wettbewerb braucht.

Begriff der WTO zur Kategorisierung von agrarpolitischen Instrumenten. Im Rahmen des AoA werden Marktzugang, inländische Stützung und Exportsubventionen unterschieden. Die inländische Stützung wiederum besteht aus drei Bereichen. Diese Bereiche werden als Boxen bezeichnet: Amber, Blue und Green Box. Die Boxen unterscheiden, wie stark ein agrarpolitisches Instrument die Produktion und damit den Handel von Nahrungsmitteln beeinflusst. In die Amber Box fallen Massnahmen, die handelsverzerrend sind. Die WTO will, dass diese langfristig abgeschafft werden. In der Blue Box sind Massnahmen zusammengefasst, welche die Produktion zwar beeinflussen, aber das Produktionslevel ist durch andere Instrumente begrenzt. Die Green Box schliesslich besteht aus Massnahmen, die keinen oder nur geringen Einfluss auf die Produktionsentscheide der Bäuerinnen und Bauern haben und dadurch auch nur minimal handelsverzerrend wirken. Dabei handelt es sich in erster Linie um die Abgeltung von öffentlichen Gütern, die von der Landwirtschaft erbracht werden. Mit Ausnahme der Versorgungssicherheitsbeiträge werden alle anderen Direktzahlungen in der Schweiz der Green Box zuzuordnen.

Die starke Erhöhung der Erträge in der Landwirtschaft durch die Einführung neuer Sorten, den Einsatz von Hilfsstoffen (Kraftfutter, Dünger und Pflanzenschutzmittel), eine verbesserte Züchtung und den Einsatz neuer Technologien zwischen 1960 und 1980 wird als Grüne Revolution bezeichnet.

Begriff aus den Politikwissenschaften. Eine Policy kann eine sogenannte Makro- und Mikroebene aufweisen. Auf der Makroebene wird die generelle Zielsetzung sowie die staatliche Auslegung festgelegt. Die Mikroebene wiederum kann als Handlungsprogramm bezeichnet werden. Dieses beschreibt die konkreten Ziele, Gesetze, Massnahmen und Instrumente. Die Schweizer Landwirtschaftspolitik 
INLÄN-
DISCHE
STÜTZUNG

INLANDLEISTUNG

INPUTORIENTIERTE/ OUTCOMEORIENTIERTE AGRARUMWELTMASSNAHMEN hat beispielsweise zum Ziel, die Biodiversität zu fördern (Policy). Das Handlungsprogramm umfasst nun verschiedene Massnahmen und Regelungen, welche die Erreichung dieses Ziels unterstützen sollen wie BFF-Beiträge, Vernetzungsbeiträge, LQB-Beiträge oder CrossCompliance-Massnahmen. Zusammen können diese Instrumente als Handlungsprogramm bezeichnet werden.

Begriff aus der WTO. Die WTO unterteilt agrarpolitische Instrumente in drei Kategorien: Marktzugang, Exportsubventionen und inländische Stützung (Domestic Support). Die inländische Stützung wiederum unterscheidet drei Boxen (Amber, Blue, Green), welche den Einfluss der Instrumente auf die landwirtschaftliche Produktion messen. Instrumente in der Amber Box beeinflussen die Produktion und wirken dadurch handelsverzerrend. Diese sollten gemäss WTO abgebaut werden. Green-Box-Instrumente beeinflussen die Produktion nicht oder nur geringfügig. Die Stützung der Landwirtschaft mit diesen Instrumenten ist im Rahmen der WTO per Definition erlaubt.

Begriff aus dem Bereich der Zollkontingente. Diese führen zu einer Importrente. Es ist deshalb wichtig zu wissen, wie die Importkontingente an die Marktteilnehmer verteilt werden. Es gibt dazu verschiedene Verfahren wie First-Come - First-Serve oder die Versteigerung. Die Inlandleistung legt in diesem Kontext fest, dass nur diejenigen von der Importrente von Zollkontingenten profitieren können, die das äquivalente Produkt auch von den inländischen Produzenten abnehmen. Die Importeure erhalten in diesem Fall ein Zollkontingent zugeteilt, das proportional zur der im Inland bezogenen Menge ist. Die Inlandleistung ist umstritten, weil sie die Marktmacht der Abnehmer verstärken kann. In der Schweiz wird daher bei der Vergabe von Zollkontingenten oft ein Mix zwischen Inlandleistung und Versteigerung angewendet (z.B. im Fleischbereich).

Charakteristiken von Agrarumweltmassnahmen: Inputorientierte Massnahmen legen eine landwirtschaftliche Produktionstechnologie oder Produktionsform als Aktivität für den Landwirt fest. Outcomeorientierte Massnahmen legen ein Ziel für den Landwirt fest und dieser kann selber entscheiden, wie er dieses Ziel erreicht, ohne dass ihm bestimmte Technologien oder Praktiken vorgeschrieben werden. Beispiele aus der Schweizer Agrarpolitik: BFF-I-Zahlungen vergüten die extensive Nutzung von Grasland. Entschädigt wird die Aktivität (1. Schnitt im Juni, keine Düngung). Im Gegensatz dazu entschädigt die BFF-II-Zahlung das Vorhandensein von spezifischen Arten auf der Wiese einer Bäuerin oder Bauern. Wann er die Wiese mäht, ist ihm überlassen. Beide Instrumente haben zum Ziel, die Biodiversität zu fördern. In der Umsetzung weisen die beiden Agrarumweltmassnahmen aber spezifische Charakteristiken auf. 
INTENSITÄT

JOINTNESS /

KOPPEL-

PRODUK-

TION

(KUPPEL-

PRODUK-

TION)

KENN-

ZEICHNUNG

KERNLAND-

WIRTSCHAFT
Verhältnis zwischen zwei Produktionsfaktoren. Zur Beschreibung der Landwirtschaft stehen zwei Arten von Intensitäten im Vordergrund. Das Verhältnis zwischen Kapital und Arbeit zeigt, wie kapitalintensiv oder mechanisiert die landwirtschaftliche Produktion ist. Das Verhältnis zwischen Kapital und Boden beschreibt den Einsatz von Hilfsstoffen pro Fläche und mögliche ökologische Probleme.

Begriff aus der rationalen Agrarpolitik. Jointness beschreibt den Umstand, dass zwei oder mehr Güter gleichzeitig produziert werden. Ein klassisches Beispiel wäre die Schafhaltung. Die Schafe produzieren gleichzeitig (jointly) Fleisch und Wolle. Man kann die Produktion dieser beiden Produkte nicht trennen; es wird immer Wolle anfallen, auch wenn man die Schafe auf eine höhere Fleischleistung züchtet. Diese gleichzeitige Produktion (oder eben Jointness in Production) kann auch auf die Bereitstellung von nicht marktfähigen Gütern übertragen werden. Dazu gehören beispielsweise die Pflege der Landschaft, die Förderung der Biodiversität oder aber auch negative Auswirkungen wie die Stickstoffauswaschung oder Ammoniakverluste. Gemeinsam ist innen, dass zwei Produkte oder Leistungen nicht unabhängig voneinander produziert werden. In der Agrarökonomie werden zwei unterschiedliche Ursachen der Jointness unterschieden. 1) Technische Ursachen sind produktionstechnische Zusammenhänge in der Landwirtschaft wie beispielsweise der Methanausstoss von Kühen. Es besteht ein zwingender agronomischer Zusammenhang zwischen Milchproduktion und Methan. 2) Ökonomische Ursachen wie beispielsweise die Nutzung eines Inputs (z.B. eine Rapsfläche), der nicht alleine zur Produktion des privaten Guts (z.B. Rapsöl) oder des öffentlichen Guts (z.B. die Landschaft) genutzt werden kann. Auch kurz- bis mittelfristig fixe Faktoren auf Betriebsebene wie die Fläche oder die Arbeit können ökonomische Gründe für die gleichzeitige Produktion oder Nicht-Produktion von nicht marktfähigen Leistungen sein.

Begriff aus dem Schweizer Landwirtschaftsgesetz. Die Kennzeichnung durch Labels oder Ursprungsbezeichnungen (AOP, AOC, IGP) soll die Qualität der Produkte signalisieren und dadurch die Transparenz und Wettbewerbsfähigkeit Schweizer Agrarprodukte erhöhen. Zudem dient die Kennzeichnung auch dem Schutz der Konsumentinnen und Konsumenten (z.B. Hinweis auf Allergene).

Als Kernlandwirtschaft gelten der Pflanzenbau und die Nutztierhaltung. Ausserdem gehören auch der Verkauf, die Aufbereitung und Lagerung selbst produzierter landwirtschaftlicher Erzeugnisse auf dem Betrieb als Kernlandwirtschaft. Kernlandwirtschaftliche Tätigkeiten werden gegenüber landwirtschaftsnahen Tätigkeiten abgegrenzt. Das Vorhandensein von kernlandwirtschaftlichen Aktivitäten 
KOFINANZIERUNG

KOMPARATIVER KOSTENVORTEIL

KONTINGENTIERUNG

LANDWIRTSCHAFTLICHER BETRIEB ist oft eine Voraussetzung für die Anwendung von agrarpolitischen Instrumenten wie Strukturverbesserungsmassnahmen oder andere Regelungen im Rahmen des BGBB oder der Raumplanung.

Kofinanzierung bedeutet, dass ein Projekt von mindestens zwei Kreditgebern finanziert wird. Im Kontext der Agrarpolitik geht es dabei um die subsidiäre Finanzierung von Zahlungen für die Landwirtschaft, d.h. für die Schweiz die Aufteilung der Kosten auf Bund und Kanton. Die meisten Instrumente der Schweizer Agrarpolitik werden durch den Bund finanziert. Einzelne Massnahmen müssen aber vom Kanton kofinanziert werden wie beispielsweise die Vernetzungsoder die Landschaftsqualitätsbeiträge. Kofinanzierung spielt eine grosse Rolle im Rahmen der EU-Agrarpolitik und der Ausgestaltung der Instrumente auf Ebene EU und Länder.

Begriff aus der Handelstheorie des Ökonomen David Ricardo. Der Begriff umschreibt, dass aus einer theoretischen Sicht Handel zwischen zwei Ländern auch dann wohlfahrtssteigernd ist, wenn ein Land sämtliche Güter kostengünstiger herstellen kann. Mit Blick auf zwei Produkte ist im Kontext dieser Theorie entscheidend, welches Produkt im Verhältnis zum anderen (d.h. komparativ) günstiger ist. Die Theorie impliziert, dass die beiden Länder sich jeweils auf das Produkt fokussieren sollten, für welches sie den komparativen Vorteil haben. Dadurch steigt theoretisch die Wohlfahrt in beiden Ländern. Empirisch kann die Theorie der komparativen Vorteile nur einen kleinen Teil des Welthandels erklären.

Die Kontingentierung ist ein agrarpolitisches Instrument zur Beschränkung von Produktionsfaktoren oder Produktionsmengen. Damit wird angestrebt, dass die Preise steigen, weil die Angebotsfunktion senkrecht geknickt wird. Die Gesamtmenge wird in Kontingente unterteilt und dann auf die Produzentinnen und Produzenten verteilt.

Der landwirtschaftliche Betrieb ist ein wichtiger Begriff in der Landwirtschaftsgesetzgebung. Er ist in der landwirtschaftlichen Begriffsverordnung folgendermassen definiert. Als Betrieb gilt ein landwirtschaftliches Unternehmen, das Pflanzenbau oder Nutztierhaltung oder beide Betriebszweige betreibt. Ein Betrieb kann mehrere Produktionsstätten, d.h. zusammenhängendes Land, Gebäude und Einrichtungen umfassen. Er muss aber rechtlich, wirtschaftlich, organisatorisch und finanziell selbstständig sowie unabhängig von anderen Betrieben sein und das ganze Jahr bewirtschaftet werden. 
LANDWIRTSCHAFTLICHES GEWERBE

LANDWIRTSCHAFTSNAHE TÄTIGKEITEN

\section{LATENTE GRUPPE}

Das landwirtschaftliche Gewerbe ist in der Schweizer Agrarpolitik ein Rechtsbegriff. Es ist eine Referenzgrösse, die in verschiedenen Gesetzen wie dem LwG, BGBB oder dem Raumplanungsgesetz zur Anwendung kommt. Das landwirtschaftliche Gewerbe ist beispielsweise eine Voraussetzung für die Übernahme des Hofes zum Ertragswert oder für den Erhalt von Investitionshilfen. Als landwirtschaftliches Gewerbe gilt in der Schweizer Landwirtschaft die Gesamtheit von landwirtschaftlichen Grundstücken, Gebäuden/Bauten und Einrichtungen/Anlagen, die der landwirtschaftlichen Produktion zugrunde liegen und mindestens über eine Standardarbeitskraft (SAK) verfügt. Die Kantone haben die Möglichkeit, die Gewerbegrenze nach unten anzupassen.

Landwirtschaftsnahe Tätigkeiten sind Produkte oder Dienstleistungen in landwirtschaftlichen Betrieben, die nicht zur Kernlandwirtschaft gehören. Landwirtschaftsnahe Tätigkeiten sind aber eng mit der Kernlandwirtschaft verknüpft wie beispielsweise der Agrotourismus, die Biomasseverwertung oder Schule auf dem Bauernhof.

Diese im Gegensatz zu gewerblichen Tätigkeiten wie das Reparieren oder Vermieten von Landmaschinen, welche keine landwirtschaftsnahen Tätigkeiten sind. Das ist vor allem aus raumplanerischer Sicht wichtig, weil gewerbliche Tätigkeiten nicht in der Landwirtschaftszone ausgeführt werden dürfen und dafür keine Gebäude oder Einrichtungen erstellt werden dürfen.

Begriff aus der Theorie des kollektiven Handelns (Collective Action). Diese Theorie beschreibt unter anderem die Organisation von Gruppen aufgrund von zwei Charakteristiken: Gruppengrösse und Homogenität. Latente Gruppen bestehen aus sehr vielen Mitgliedern mit einer relativ grossen Heterogenität der Interessen. Das bedeutet, dass sie sich aus einer theoretischen Perspektive nur schwer organisieren lassen. Das klassische Beispiel für eine latente Gruppe in der Agrarpolitik sind die Konsumenten. Die Interessen der Konsumenten lassen sich nur schwer organisieren und damit politisch vertreten, weil es sehr viele Konsumentinnen und Konsumenten gibt und diese ganz unterschiedliche Ansprüche an Nahrungsmittel haben können (Preis, Qualität, Umweltstandards etc.). Auch Produzentenorganisationen wie beispielsweise der Schweizerische Bauernverband (SBV) haben oft den Charakter von latenten Gruppen. Diese versuchen über Nebenprodukte (Nebenprodukttheorie) Mitglieder und damit politischen Einfluss zu gewinnen. 
LENKUNGSABGABE

LOBBYING

MARKTMACHT
Eine Lenkungsabgabe ist eine Steuer, deren Einnahmen in den besteuerten Sektor zurückfliesst. Mit der Besteuerung von Pflanzenschutzmitteln könnten beispielsweise präzise Applikationstechnologien subventioniert werden, die den Eintrag von Pflanzenschutzmitteln reduzieren. Lenkungsabgaben sollen daher nicht nur den Einsatz von Inputs reduzieren, welche negative externe Effekte haben, sondern auch dem landwirtschaftlichen Sektor dabei unterstützen, diese Reduktion mithilfe von Technologie oder alternativen Produktionsmöglichkeiten zu kompensieren.

Lobbying bezeichnet die Interessenvertretung in der Politik. In der Agrarpolitik gibt es eine Vielzahl von Verbänden und Organisationen, die versuchen, den politischen Entscheidungsprozess zu ihren Gunsten zu beeinflussen. Der Einfluss hängt von der Grösse und der politischen Macht (Referendumsmacht) ab. Aus der Sicht der neuen politischen Ökonomie versuchen die Interessenvertreter die Entscheidungen so lange zu beeinflussen, bis die Grenzkosten des Lobbyings grösser sind als der Grenznutzen, der aus den politischen Entscheiden erfolgt. Man spricht in diesem Zusammenhang von RentSeeking. Dadurch werden Produktionsfaktoren (Arbeit und Kapital) in den politischen Prozess investiert, die aus einer ökonomischen Sicht in anderen Bereichen produktiver wären.

Marktmacht beschreibt eine Marktsituation, in welcher der Wettbewerb fehlt oder eingeschränkt ist. Es gibt verschiedene Ausprägungen von Marktmacht. Am stärksten wird der Wettbewerb in einer Monopolsituation eingeschränkt. In diesem Fall gibt es ein vorherrschendes Unternehmen, welches den Preis und die angebotene Menge in einem Markt bestimmen kann. Aber auch Oligopole (Angebot von wenigen) oder Oligopsone (Nachfrage von wenigen) führen zu einem eingeschränkten Wettbewerb. Marktmacht benachteiligt andere Marktteilnehmer (Produzenten oder Konsumenten). Im Kontext der Schweizer Agrarpolitik ist es wichtig, dass die Wertschöpfungskette für Nahrungsmittel in der Schweiz eine XStruktur aufweist und dadurch Wettbewerbsverzerrungen entstehen können. So vereinen die beiden grossen Detailhändler in der Schweiz fast $70 \%$ des Marktes für Lebensmittel. Man kann in diesem Zusammenhang von einem Teiloligopol sprechen. Wie gross die Marktmacht allerdings tatsächlich ist, lässt sich nur empirisch bestimmen. Die Agrarpolitik versucht mithilfe der gesetzlichen Regelung von Branchen- und Produzentenorganisationen der Marktmacht der Abnehmer entgegenzuwirken. 
MARKTORDNUNG

MARKTVERSAGEN

MIT-

NAHMEEFFEKT

MOTION, POSTULAT, INTERPELLATION, PARLAMENTARISCHE INITIATIVE
Die Marktordnung umschreibt, wie Angebot, Nachfrage und Preise in einem bestimmten landwirtschaftlichen Markt durch staatliche Rahmenbedingungen gesteuert werden. Die Marktordnung kann sich zwischen einzelnen landwirtschaftlichen Produkten mit unterschiedlichen Eigenschaften stark unterscheiden (z.B. zwischen Milch, Fleisch, Eier und Gemüse). Sie umfasst dabei nicht nur die einzelnen gesetzlichen Regelungen, sondern ist auch stark durch die Anzahl und Zusammensetzung der Marktakteure bestimmt. Die Produzenten- und Branchenorganisationen sind Schlüsselakteure innerhalb der Marktordnung.

Begriff aus der rationalen Agrarpolitik. Marktversagen beschreibt eine Situation, in der eine Marktlösung nicht zur optimalen Allokation der Ressourcen führt. Die Gründe dafür sind vielfältig z.B. externe Effekte, öffentliche Güter, unvollständiger Wettbewerb (Monopol) etc. Aus einer ordnungspolitischen Sicht ist Marktversagen eine notwendige Voraussetzung für einen staatlichen Eingriff. Marktversagen alleine ist aber keine hinreichende Voraussetzung für staatliche Eingriffe in den Markt. Die zweite Bedingung ist, dass die politischen Massnahmen auch zu einer Verbesserung der Ressourcenallokation führen (im Sinne eines Paretooptimums). Ansonsten kann durch die staatliche Massnahme auch Politikversagen entstehen.

Art von Politikversagen: Ein finanzieller Anreiz führt zu keiner Verhaltensänderung beim Politikadressaten. Von Mitnahmeeffekten spricht man, wenn beispielsweise die Landwirte finanziell entschädigt werden für etwas, das sie auch ohne Stützung tun würden. Mitnahmeeffekte reduzieren die Effizienz einer Politikmassnahme erheblich und können im Extremfall dem Politikversagen zugeordnet werden, weil sich die Ressourcenallokation verschlechtert.

Parlamentarische Begriffe: Mit einer Motion erhält der Bundesrat den Auftrag, einen Entwurf zu einem Erlass der Bundesversammlung vorzulegen oder eine Massnahme zu treffen. Sie verlangt daher vom Bundesrat, eine Gesetzesänderung vorzuschlagen. Ein Postulat hingegen beauftragt den Bundesrat nur zu prüfen und zu berichten, ob ein Entwurf zu einem Erlass der Bundesversammlung vorzulegen oder eine Massnahme zu treffen sei. Sie ist damit weniger verbindlich für den Bundesrat, gesetzlich aktiv zu werden. Mit einer Interpellation schliesslich wird vom Bundesrat Auskunft über wichtige innen- und aussenpolitische Ereignisse und Angelegenheiten des Bundes verlangt. Parlamentarierinnen und Parlamentarier haben mit der parlamentarischen Initiative die Möglichkeit, individuell oder gemeinsam mit der Fraktion oder einer Kommission einen Vorschlag für ein Gesetz oder eine Verfassungsänderung zu machen. 
MULTIFUNKTIONALITÄT GEMÄSS VERFASSUNG

MULTIFUNKTIONALITÄT: ÖKONOMISCHE DEFINITION

NACHFRAGE

NACH

ÖFFENT-

LICHEN

GÜTERN

NACHHALTIGKEIT

NAHRUNGS-

MITTELSICHERHEIT

NEBENPRODUKTTHEORIE
Die multifunktionalen Aufgaben der Landwirtschaft sind im Artikel 104 der Bundesverfassung festgehalten. Die Landwirtschaft soll, neben einer nachhaltigen und auf den Markt ausgerichteten Produktion, einen wesentlichen Beitrag leisten zur a) sicheren Versorgung der Bevölkerung; b) Erhaltung der natürlichen Lebensgrundlagen und zur Pflege der Kulturlandschaft sowie c) dezentralen Besiedlung des Landes.

Multifunktionalität aus einer agrarökonomischen Sicht wird definiert durch zwei simultane Bedingungen. Einerseits muss eine Koppelproduktion vorliegen (Jointness in Production). Andererseits muss ein Marktversagen vorliegen, d.h., das Koppelprodukt der landwirtschaftlichen Produktion muss den Charakter einer Externalität oder eines öffentlichen Guts aufweisen.

Im Gegensatz zu privaten Gütern werden die individuellen Zahlungsbereitschaften für öffentliche Güter vertikal und nicht horizontal aggregiert. Dadurch wird die angebotene Menge nicht über den Preis, sondern die Menge festgelegt. Dieser Unterschied führt dazu, dass der Beitrag jedes Einzelnen zum öffentlichen Gut sehr klein wird und dadurch die Bereitschaft, die Kosten mitzutragen, sinkt.

Nachhaltige Entwicklung ist eine Entwicklung, die die Bedürfnisse der Gegenwart befriedigt, ohne zu riskieren, dass künftige Generationen ihre eigenen Bedürfnisse nicht befriedigen können (Definition Brundtland-Report). Der Begriff der Nachhaltigkeit bezieht sich im Grundsatz immer gleichzeitig auf die Bereiche Umwelt, Gesellschaft und Wirtschaft.

Nahrungsmittelsicherheit (Food Safety) umschreibt den Umstand, dass durch den Verzehr von Lebensmitteln keine Schädigungen oder gesundheitlichen Beeinträchtigungen entstehen. Um Nahrungsmittelsicherheit sicherzustellen, sieht die Agrarpolitik verschiedene Massnahmen vor wie beispielsweise Hygienekontrollen in der Produktion, Messung von Rückständen in Lebensmitteln oder die Sicherstellung der Rückverfolgbarkeit von Lebensmitteln und dessen Bestandteilen.

Die Nebenprodukttheorie ist ein Begriff aus der neuen politischen Ökonomie. Die Organisation von Gruppen zur Durchsetzung von kollektiven Interessen ist aufgrund der Problematik des Trittbrettfahrens oft schwierig. Die Nebenprodukttheorie impliziert, dass zur Stärkung des kollektiven Handelns (Collective Action) die Interessenorganisation ein privates Gut anbieten sollte, damit möglichst viele Betroffene sich beteiligen. Die politische Vertretung der gemeinsa- 
NEUE POLITISCHE ÖKONOMIE

NICHT TARIFÄRE HANDELSHEMMNISSE

NON-POINT SOURCE POLLUTION

NORMATIV UND POSITIV men Interessen wird damit zum Nebenprodukt. Der Schweizer Bauernverband bietet beispielsweise Versicherungs- und Beratungsleistungen an.

Die neue politische Ökonomie (auch Public oder Social Choice) beschäftigt sich mit der Interpretation politischer Entscheidungen durch die Anwendung ökonomischer Konzepte. Die Grundannahme ist, dass den Akteuren ein Streben nach einer individuellen Nutzenmaximierung und ein rationales Verhalten zugeschrieben wird. Auf dieser Basis wird das kollektive und individuelle Verhalten von politischen Akteuren und Interessengruppen analysiert.

Nicht tarifäre Handelshemmnisse sind Massnahmen, die den freien Warenverkehr zwischen Ländern einschränken und nicht Zölle, Zollkontingente oder Exportsubventionen sind. Dazu gehören beispielsweise sanitäre und phytosanitäre Massnahmen (wie Kontrolle von Antibiotika oder Pestiziden in Nahrungsmitteln), technische Schutzmassnahmen (wie Beschriftung der Produkte in allen Landessprachen) oder Quarantänemassnahmen (z.B. bei lebenden Tieren). Auch Herkunftsangaben werden, wenn nicht gegenseitig anerkannt, als nicht tarifäre Handelshemmnisse gesehen. Agrarexporteure kritisieren Länder, die nicht tarifäre Handelshemmnisse aufbauen, dass diese letztendlich nur dem Schutz der eigenen Produktion dienen (Protektionismus). Agrarimporteure argumentieren oft mit dem Vorsorgeprinzip, insbesondere mit Blick auf sanitäre und phytosanitäre Massnahmen.

Non-Point Source Pollution ist eine Bezeichnung für umweltverschmutzende Stoffe, die aus mehreren und diffusen Quellen hervorgehen können. Beispiele für Non-Point Source Pollution in der Landwirtschaft sind Dünger, Herbizide, Insektizide, die durch Wind, Niederschlag und andere Vektoren in ökologische Systeme, wie Seen, Flüsse oder ins Grundwasser, gelangen. Die Rückverfolgbarkeit dieser Hilfsstoffe in natürlichen Systemen zu ihrer Quelle (z.B. einem Landwirt) ist nur schwer oder nicht möglich. Dadurch lässt sich das Verursacherprinzip auf diese Umweltprobleme nicht oder kaum anwenden.

Normativ bedeutet, dass erörtert wird, wie etwas sein sollte. Eine normative Perspektive beinhaltet zwangsläufig eine Definition der zugrunde liegenden Wertvorstellungen. Der normative Fokus bezieht sich in diesem Buch auf wohlfahrtsökonomische Grundlagen (Marktwirtschaft und rationales Verhalten). Positiv hingegen bezieht sich auf die Beschreibung, wie es tatsächlich ausgestaltet ist. Die tatsächliche Ausgestaltung der Agrarpolitik weicht normalerweise von 
OECD

ÖFFENTLICHES GUT

der normativen Perspektive ab, weil unterschiedliche Wertvorstellungen und Ziele in den agrarpolitischen Prozessen zusammenkommen. Beide Perspektiven ergänzen sich und sind wichtig für das Verständnis der Agrarpolitik.

Die OECD ist die Organisation für wirtschaftliche Zusammenarbeit und Entwicklung. Sie umfasst 36 demokratische und marktwirtschaftlich orientierte Mitgliedstaaten. Der Zweck der Organisation ist, die Mitgliedsländer zu beraten. Dabei sollen Erfahrungen über Politikansätze und Politikstrategien ausgetauscht und internationale Politik koordiniert werden. Analysen und Empfehlungen der OECD appellieren an eine liberale, marktwirtschaftliche und effiziente Wirtschaftsordnung. Sie setzt aber auch Standards für Direktinvestitionen, Steuersysteme oder im Kampf gegen die Geldwäsche. Mit Blick auf die Landwirtschaft ist die länderübergreifende Beurteilung der Agrarpolitiken von grosser Bedeutung.

Ein öffentliches Gut wird über zwei Kriterien definiert. Einerseits kann niemand vom Konsum des Guts oder der Leistung ausgeschlossen werden (Ausschliessbarkeit). Andererseits besteht keine Rivalität im Konsum des Guts (Nicht-Rivalität). Klassisches Beispiel für ein öffentliches Gut der Landwirtschaft ist die Kulturlandschaft. Niemand kann von der Betrachtung der Landschaft ausgeschlossen werden und die Schönheit der Landschaft geht auch nicht zurück, wenn mehr Leute das Landschaftsbild anschauen (d.h. konsumieren). Öffentliche Güter werden unter marktwirtschaftlichen Bedingungen nicht erbracht und verursachen unter gewissen Bedingungen daher ein Marktversagen. Dieses Marktversagen ist in vielen Fällen die Rechtfertigung für staatliche Eingriffe in die Agrarwirtschaft. Es gilt zu beachten, dass die Definition von öffentlichen Gütern verschiedene Abstufungen kennt.

ÖKOLOGI- Der ökologische Leistungsnachweis ist ein Anforderungskriterium für SCHER LEISTUNGSNACHWEIS den Erhalt von Direktzahlungen in der Schweiz. Dieser Nachweis entspricht einer sogenannten Cross-Compliance Massnahme (Bindung der Direktzahlung an einen Standard). Der ökologische Leistungsnachweis legt im Kern fest, dass die Bestimmungen des Tier- und Gewässerschutzes eingehalten werden und eine ausgeglichene Nährstoffbilanz ausgewiesen wird. Darüber hinaus braucht es einen angemessenen Anteil an Biodiversitätsförderflächen (7\%) sowie eine geregelte Fruchtfolge und einen geeigneten Bodenschutz. 
OPPORTUNITÄTSKOSTEN

ORDNUNGSPOLITIK

OUTPUT, OUTCOME, IMPACT

PARETOEFFIZIENZ

PLANETARY BOUNDARIES
Die Opportunitätskosten von Bäuerinnen und Bauern sind entgangene Erlöse aus einer alternativen landwirtschaftlichen Nutzung. Wenn der Landwirt eine Biodiversitätsförderfläche ausscheidet, dann hat er direkte Kosten, welche ihm durch das extensive Management entstehen. Zudem fallen aber auch Opportunitätskosten an. Wenn er die Fläche intensiv nutzen würde (z.B. als intensive Wiese), dann hätte er aus dem Nettoerlös des Grases einen Gewinn erzielt. Dieser Betrag, also das entgangene Einkommen aus einer alternativen Nutzung, entspricht den Opportunitätskosten.

Begriff aus der normativen Agrarökonomie. Ordnungspolitik umschreibt eine Wirtschaftsordnung, die nach den Prinzipien von Markt und Wettbewerb organisiert wird. Das Ziel ist, freie wirtschaftliche Tätigkeit, Schaffung von Märkten und Wettbewerb. Staatliche Eingriffe werden nur bei Marktversagen als legitim betrachtet.

Begriffe aus der Evaluation von Politikmassnahmen. Output, Outcome und Impact sind drei Stufen, um die Umsetzung einer Massnahme oder eines Instruments zu beurteilen. Output umfasst die erste Wirkungsstufe und beschreibt, ob die Massnahme eine gewünschte Verhaltensänderung bei den Politikadressaten erreicht. Der Outcome beschreibt die Zielerreichung auf der zweiten Wirkungsstufe. Evaluiert wird, wie gross der Beitrag dieser Verhaltensänderung zur Lösung des zugrunde liegenden Problems ist. Impact schliesslich beschreibt inwiefern sich das Programm/Instrument langfristig auf das gesellschaftliche Problem auswirkt. Auf dieser dritten Wirkungsstufe werden auch Nebeneffekte oder andere Handlungsprogramme in die Beurteilung der Politikmassnahme miteinbezogen. Vorsicht: unterschiedliche Disziplinen wie Politikwissenschaften oder Agrarökonomie verwenden manchmal unterschiedliche Begriffsdefinitionen für Output, Outcome und Impact.

Begriff aus der Wohlfahrtsökonomie. In einem Paretooptimum kann durch eine Verschiebung der Ressourcenallokation niemand bessergestellt werden, ohne dass ein anderer Marktteilnehmer schlechtergestellt wird. Paretoeffizienz bestimmt dadurch eine effiziente Ressourcenallokation. Sie sagt aber nichts über die Verteilungsgerechtigkeit aus.

Wissenschaftliches Konzept zur Sichtbarmachung der Endlichkeit von natürlichen Ressourcen. Die Idee ist, dass, wenn die planetarischen Grenzen überschritten werden, die Stabilität und Resilienz der Erde erheblich gefährdet ist. Die Landwirtschaft und die Produktion von Nahrungsmitteln steht in Zusammenhang mit verschiedenen planetarischen Grenzen (Klima, Biodiversität, Stickstoff, Gewässerverschmutzung, Regenwaldrodung etc.). Das Konzept fasst dadurch 
POLICY, POLITY, POLITICS

POLITIKVERSAGEN

POLITIKZYKLUS

PREISDIFFERENZIERUNG

PREISSTANDARDANSATZ die Wechselwirkung zwischen Landwirtschaft und Umwelt zusammen, die ein wichtiger Treiber für agrarpolitische Entwicklungen weltweit ist.

Polity umfasst die Form oder Struktur der Politik und bezieht sich auf institutionelle Aspekte (Regierungssysteme, Parlamente, politische Parteien etc.) und auf deren Rechtsordnung (Verfassung, Gesetze etc.). Policy beinhaltet die Aufgaben und Ziele, welche die Politikakteure formulieren und realisieren wollen (Handlungsprogramme, Gesetze, Verordnungen etc.). Politics fokussiert auf Prozesse, wie politische Verfahren ablaufen (z.B. Gesetzgebungsprozesse, Abstimmungen, Lobbyismus), und darauf, wie Interessengruppen ihre Anliegen durchzusetzen suchen.

Führt eine Politikmassnahme nicht zu einer wohlfahrtsökonomischen Verbesserung der Ressourcenallokation, dann spricht man aus einer ordnungspolitischen Sicht von Politikversagen. Das bedeutet, die Massnahme hat nicht zum gewünschten Effekt geführt oder zu massiven Nebeneffekten geführt, welche die eigentliche Wirkung der Massnahme überkompensierten.

Begriff aus der Politikwissenschaft. Beschreibt die Politik (Politics) als Abfolge von Phasen des politischen Prozesses. Grundsätzlich können fünf Phasen unterschieden werden: Problemdefinition, AgendaSetting, Politikformulierung, Politikimplementierung und Politikevaluierung. Unter idealen Voraussetzungen kann nach Beendigung des Zyklus eine Politik auch terminiert werden. Normalerweise beginnt jedoch der Zyklus von vorne, d.h., Handlungsprogramme oder politische Massnahmen durchlaufen den Prozess mehrmals.

Die Preisdifferenzierung ermöglicht es einem Anbieter, für die gleiche Leistung unterschiedliche Preise festzulegen. Damit kann die Zahlungsbereitschaft unterschiedlicher Segmente von Konsumentinnen oder Abnehmern ausgenutzt werden, um die Profitabilität zu erhöhen. Ausschlaggebend ist, dass Marktsegmente existieren, die unterschiedlichen Elastizitäten aufweisen, und voneinander getrennt werden können.

Der Preis-Standard-Ansatz ist ein Instrument aus der (Agrar-)Umweltpolitik. Das Ziel ist die Internalisierung von externen Effekten durch die Festlegung von Preis (z.B. einer Direktzahlung) und Umweltstandard (z.B. jeder Betrieb muss 7 \% Biodiversitätsförderflächen aufweisen). Die Politik passt Preise oder Standards den Entwicklungen der Umweltqualität und den politischen Zielen an. 
PRINCIPLE-

AGENT-

PROBLE-

MATIK

PRIVAT-

RECHTLICH, ÖFFENTLICHRECHTLICH

PRODUKTIVITÄT

PRODU-

ZENTEN-

ORGANISA-

TION
Die Principle-Agent-Problematik bezieht sich auf die Informationsasymmetrie zwischen einem Auftraggeber und einem Auftragnehmer. Im Kontext der Agrarpolitik bezieht sich dies in erster Linie auf das Verhältnis zwischen dem staatlichen Regulator (Principle) und den Bäuerinnen und Bauern (Agents). In der Regel haben der Principle und der Agent nicht die gleichen Interessen und der Agent hat gegenüber dem Principle meist einen Wissensvorsprung, den er zu seinen Gunsten nutzen kann. Daraus entstehen spezifische Probleme wie «Moral Hazard» oder «Adverse Selection», die für die Wirkungsweise von agrarpolitischen Massnahmen wichtig sein können. Ein Beispiel für "Adverse Selection» wäre, wenn die Bäuerinnen und Bauern nur für die Biodiversität ungeeignete Flächen für das BFFProgramm anmelden würden.

Das Privatrecht regelt rechtliche Beziehungen zwischen Privatpersonen, während das öffentliche Recht die Beziehung zwischen der öffentlichen Gewalt und den Bürgern regelt. In der Landwirtschaftspolitik spielt diese Unterscheidung in Bezug auf das BGBB eine wichtige Rolle, weil dieses sowohl privatrechtliche (z.B. Betriebsübernahme innerhalb der Familie) als auch öffentlich-rechtliche Regelungen (familienexterne Regelungen) umfasst.

Produktivität beschreibt das Verhältnis von Output zu Input wie Arbeit, Kapital oder Boden. Die Arbeitsproduktivität beispielsweise beschreibt die Menge an produzierten Gütern pro Arbeitsstunde (z.B. kg Milch pro h). Die Flächenproduktivität und die Kapitalproduktivität beschreiben die produzierte Menge pro Fläche und Investition. Die Produktivität lässt sich von der Intensität unterscheiden, welche das Verhältnis zwischen zwei Inputfaktoren beschreibt (z.B. Kapital pro Fläche). Die Produktivität der Landwirtschaft ist weltweit sehr heterogen und es gibt Regionen mit einer hohen Arbeits-, aber geringen Flächenproduktivität oder umgekehrt. Die Produktivitätssteigerung in der Landwirtschaft, unter anderem in der Grünen Revolution, ist ein wichtiger Treiber der Agrarpolitik weltweit.

Eine Produzentenorganisation (PO) ist ein Zusammenschluss von Bäuerinnen und Bauern, die das gleiche Produkt oder die gleiche Produktgruppe herstellen. Das Ziel von Produzentenorganisationen ist die Interessenvertretung auf dem Markt und in der Politik. Auf dem Markt kann das Angebot durch die Produzentenorganisation unterstützt und koordiniert werden. Es können sogenannte Selbsthilfemassnahmen umgesetzt werden. Dazu gehören beispielsweise Absatzförderungs- und Vermarktungsaktionen zugunsten der inländischen Produktion, Verbesserung der Transparenz im Markt, die Ausarbeitung von Standardverträgen oder die Anpassung des Ange- 
PRODUZENTEN- UND KONSUMENTENRENTE

PROJEKTORIENTIERTE MASSNAHMEN bots an den Markt. Auf der politischen Ebene vertreten die Produzentenorganisationen das Interesse ihrer Mitglieder. Beispiele für Produzentenorganisationen: Schweizerischer Getreideproduzentenverband (SGPV), Schweizer Milchproduzenten (SMP), Verband Schweizer Gemüseproduzenten (VSGP) oder die Suisseporc. Neben den Produzenten gibt es auch Organisationen für Händler und Verarbeiter von landwirtschaftlichen Produkten. Gemeinsame Interessen verfolgen diese unterschiedlichen Organisationen in der sogenannten Branchenorganisation (BO). Diese können ebenfalls Selbsthilfemassnahmen umsetzen. Beispiele für Branchenorganisationen: Proviande oder Branchenorganisation Milch.

In einem vollkommenen Markt bildet sich der Preis für Agrargüter dort, wo sich Angebot, d.h. die horizontal aggregierte Grenzkostenkurve der Landwirte, und Nachfrage, d.h. die horizontale Aggregation der individuellen Zahlungsbereitschaft der Konsumenten bei gegebenem Budget, schneiden. Zwischen dem Marktpreis und der Zahlungsbereitschaft der Konsumenten ergibt sich die sogenannte Konsumentenrente. Diese ist die Summe der Zahlungsbereitschaften derjenigen Konsumenten, die auch bereit gewesen wären, einen höheren Produzentenpreis zu bezahlen. Umgekehrt fasst die Produzentenrente denjenigen Gewinn der Landwirte zusammen, der zwischen der Angebotskurve und dem Marktpreis liegt; d.h. derjenigen Landwirte, die auch zu tieferen Preisen kostendeckend produzieren können.

Option für die Ausgestaltung eines agrarpolitischen Instruments. Der Fokus liegt darauf, dass das agrarpolitische Instrument nicht auf individuelle Politikadressaten (d.h. jede Bäuerin einzeln) ausgerichtet ist, sondern auf ein Kollektiv. Entsprechend müssen sich Bäuerinnen und Bauern und andere Akteure zusammenschliessen und ein Projekt erarbeiten, um vom entsprechenden Instrument (einer Direktzahlung oder Investitionshilfe) profitieren zu können. Das Projekt wird durch die Administration geprüft und finanziert. Beispiele für solche projektorientierte Massnahmen in der Schweizer Agrarpolitik sind Vernetzungsprojekte (Vernetzungsbeitrag), Landschaftsqualitätsprojekte (LQB-Beitrag), aber auch Projekte der regionalen Entwicklung (PRE).

Das Erzeugerstützungsmass PSE (Producer Support Estimate) ist ein Indikator, der die Unterstützung der landwirtschaftlichen Produktion durch den Konsumenten und Steuerzahler misst. Das «Percentage PSE» sagt aus, wie gross der Anteil der staatlichen Stützung am Einkommen der Landwirte ist. In der Schweiz lag dieser Wert in den letzten Jahren zwischen 50 und $60 \%$. Das heisst, von jedem Franken, den ein Landwirt verdiente, entspringen 50 bis 60 Rappen der 
QUALITÄTSSTRATEGIE

RELEVANZ, EFFIZIENZ, EFFEKTIVITÄT

RENTSEEKING staatlichen Stützung. Ausserdem unterscheidet das PSE, wie stark die einzelnen Instrumente die landwirtschaftliche Produktion beeinflussen. Die Idee dahinter ist, dass Instrumente, welche die Produktion von landwirtschaftlichen Gütern stark beeinflussen, aus einer wirtschaftsliberalen Perspektive weniger geeignet sind, die Landwirtschaft zu unterstützen.

Die Produktionskosten der Schweizer Landwirtschaft sind im Vergleich zu anderen Ländern hoch. Die Idee der Qualitätsstrategie ist es, die Anforderungen an die Bäuerinnen und Bauern im Bereich Tierwohl, Umwelt und Nahrungsmittelsicherheit auf Märkten in Wert zu setzen. Dadurch soll die Wettbewerbsfähigkeit der Schweizer Landwirte gestärkt werden. Das bedeutet, dass die Produktion auf qualitativ hochwertige Produkte gefördert werden soll, um dem Preiswettbewerb auf den Agrarmärkten auszuweichen. Wichtige agrarpolitische Instrumente zur Unterstützung der Qualitätsstrategie sind Labels und Kennzeichnungen, wie Herkunft, Produktionsart oder Ursprungskennzeichnungen (AOP, AOC, IGP).

Begriffe aus der Politikevaluation. Relevanz: Verhältnis zwischen dem übergeordneten agrarpolitischen Ziel und dem Ziel, welches mit einem bestimmten Handlungsprogramm oder agrarpolitischen Instrument angestrebt wird. Effizienz: Verhältnis zwischen den eingesetzten Ressourcen und dem Output, Outcome oder Impact eines Handlungsprogramms oder Instrument. Achtung: Die Begriffe Output, Outcome und Impact können je nach Wissenschaftsdisziplin unterschiedliche Wirkungsstufen implizieren. Effektivität: Grad der Zielerreichung, d.h. wie gut ein Programm oder eine Massnahme zur Erreichung eines Ziels auf Stufe Output, Outcome oder Impact beiträgt.

Rent-Seeking ist ein Einkommen (Rente), das eine Interessengruppe oder Branche durch politisches Lobbying erzielt. Mit anderen Worten, man verwendet Ressourcen (Arbeit, Kapital), um wirtschaftspolitische Privilegien zu erhalten. Rent-Seeking ist aus einer ordnungspolitischen Sicht ineffizient, weil es einerseits Ressourcen bindet, die in der Produktion von Marktgütern fehlen. Andererseits entsteht die Rente auf Kosten anderer. Beispielsweise man erhöht die Produzentenrenten und schmälert gleichzeitig die Konsumentenrente. In der Agrarpolitik kann Rent-Seeking Verhalten beispielsweise erklären, weshalb trotz wohlfahrtsökonomischen Nachteilen der Grenzschutz ein zentrales Instrument ist. 
SAK

SECOND-

BEST THEORY

SEKTORALE

WIRT-

SCHAFTSPOLITIK
Die Standardarbeitskraft (SAK) ist ein Rechtsbegriff in der Schweizer Agrarpolitik. Sie repräsentiert eine objektive Messgrösse, um die Grösse eines landwirtschaftlichen Betriebes in der Schweiz zu bestimmen. Landwirtschaftliche Betriebe können nur dann von Privilegien wie beispielsweise den Direktzahlungen, den Strukturverbesserungsmassnahmen oder den Vorteilen des bäuerlichen Bodenrechts profitieren, wenn sie eine gewisse Grösse (in SAK) aufweisen. Eine Standardarbeitskraft (SAK) umfasst 2600 Arbeitskraftstunden pro Jahr. Wichtig: Die SAK ist nicht geeignet, den effektiven Arbeitsaufwand in einem Betrieb abzubilden, sondern stellt ein standardisiertes Mass für die kernlandwirtschaftlichen Aktivitäten dar. Die Arbeitszeit, die für die Berechnungen der SAK-Faktoren beigezogen wird, basiert auf dem detaillierten Arbeitsvoranschlag der Agroscope und beinhaltet sämtliche Arbeitskategorien (inklusive Betriebsmanagement und Sonderarbeiten).

Begriff aus der Wohlfahrtsökonomie. Ökonomische Modelle setzen verschiedene Annahmen voraus. Viele bedingen, dass ein System im Gleichgewicht ist (Equilibrium). Beispielsweise entsprechen im ökonomischen Optimum die Grenzkosten dem Preis für das Produkt. Aus einer ordnungspolitischen Perspektive gilt in diesem Gleichgewicht immer, dass Laisser-faire die bestmögliche Politik ist und es kein Eingreifen des Staates in den Markt braucht. Wenn aber bestimmte Voraussetzungen eines funktionierenden Marktes verletzt werden, dann kann die grösste mögliche Wohlfahrt nicht mehr erreicht werden. Die Second-Best Theory (die Theorie des Zweitbesten) besagt nun, dass politische Interventionen in diesem Fall zwar nicht das globale Optimum herbeiführen, aber wesentlich dazu beitragen können, dass die Wohlfahrt im Vergleich zur Marktsituation erhöht werden kann. Das Prinzip kommt insbesondere im Kontext des Agrarhandels zur Anwendung. Aus einer wohlfahrtsökonomischen Perspektive würde multilateraler Freihandel die Wohlfahrt weltweit erhöhen. Gegeben, dass andere Länder aber ihre Grenzen schützen und die landwirtschaftlichen Wertschöpfungsketten nicht zwangsläufig im Gleichgewicht sind, können Länder mit eigenem Grenzschutz die Wohlfahrt trotzdem steigern.

Die Agrarpolitik ist im Grundsatz eine sektorale Wirtschaftspolitik. Das heisst, sie regelt im Wesentlichen die Marktordnung für landwirtschaftliche Produkte und schafft so die Voraussetzung für eine ökonomisch nachhaltige und krisenresistente Nahrungsmittelproduktion in Inland. Die Agrarpolitik hat aber verschiedene Verbindungen in andere Politikbereiche wie Umweltschutz, Raumplanung, Konsumentenschutz oder Regionalpolitik. Diese Verbindungen sind 
SELBSTBEWIRTSCHAFTER

SELBSTHILFE

SELBSTVERSORGUNGSGRAD

SOZIALE

BEGLEITMASSNAHMEN aber nicht in einer ganzheitlichen Gesetzgebung integriert (Food Policy), sondern werden ad hoc aufeinander abgestimmt.

Begriff aus dem Bundesgesetz über das bäuerliche Bodenrecht. Selbstbewirtschafter ist eine Person, die den Boden selber bearbeitet, und wenn es sich um ein landwirtschaftliches Gewerbe handelt, dieses persönlich leitet. Der Begriff des Selbstbewirtschafters stellt sicher, dass nur Bäuerinnen und Bauern tatsächlich landwirtschaftliche Flächen oder Betriebe erwerben können.

Begriff aus dem Schweizer Landwirtschaftsgesetz. Das Prinzip der Selbsthilfe impliziert, dass die Förderung der Qualität und des Absatzes sowie die Anpassung der Produktion und des Angebotes an die Erfordernisse des Marktes Aufgaben der Produzenten und Produzentinnen oder der entsprechenden Branchen sind. Auf diese Weise wird ein Teil der Verantwortung für die Regelungen der Marktordnungen an die Landwirtschaft übertragen. Damit soll verhindert werden, dass der Staat zu stark in die marktwirtschaftlichen Kräfte von Angebot und Nachfrage eingreift.

Der Selbstversorgungsgrad beschreibt das Verhältnis zwischen der landwirtschaftlichen Produktion im Inland (gemessen in verwertbarer Energie) zum Gesamtverbrauch im Inland. Der Gesamtverbrauch wird berechnet, indem man die Produktion um die Importe, Exporte und Vorratsveränderungen korrigiert. Es wird zwischen einem Brutto- und einem Netto-Selbstversorgungsgrad unterschieden. Der Netto-Selbstversorgungsgrad berücksichtigt dabei, dass ein Teil der Inlandproduktion auf importierten Futtermitteln beruht. In der Schweiz liegt der Brutto-Selbstversorgungsgrad bei ungefähr $60 \%$, der Netto-Selbstversorgungsgrad zwischen 50 und $55 \%$. Es gilt zu beachten, dass der Selbstversorgungsgrad sowohl von der inländischen Produktion als auch der Veränderung der Bevölkerung beeinflusst wird.

In der Schweizer Agrarpolitik werden die Betriebshilfe und die Umschulungsbeihilfe als soziale Begleitmassnahmen bezeichnet. Mit der Betriebshilfe, einem Investitionskredit, werden bäuerliche Betriebe unterstützt, die unverschuldet oder durch Veränderung der wirtschaftlichen Rahmenbedingungen in eine finanzielle Notlage geraten sind (z.B. bei Unwetter, Krankheit, Unglücksfall). Die Umschulungsbeihilfe hatte zum Ziel, Bäuerinnen und Bauern in der Umschulung zu unterstützen. Diese Massnahme wurde Ende 2019 aufgrund fehlender Nachfrage aufgegeben. 
STANDARDVERTRAG

STRUKTURELLE TRANSFORMATION

STRUKTURVERBESSERUNG

TAILORING

TARGETING
Ein Standardvertrag dient zu einer einheitlichen Regelung der Gegebenheiten eines Geschäfts innerhalb einer Branche. Im Landwirtschaftssektor gibt es verschiedene Standardverträge, welche die Grundlagen für den Kauf und Verkauf von landwirtschaftlichen Produkten regelt. Damit soll der Marktmacht der Abnehmer im Landwirtschaftssektor entgegengewirkt werden. Ein zentraler Standardvertrag gilt für die Milchbranche, welcher für die Akteure in der Wertschöpfungskette Milch einheitliche Rahmenbedingungen schafft und somit zu Verbindlichkeit und Transparenz führen soll. Er stärkt dadurch die Position des Milchverkäufers (d.h. der Bäuerinnen und Bauern).

Die strukturelle Transformation beschreibt den Wandel von einer landwirtschaftlich geprägten Wirtschaft hin zu einer Dienstleistungsgesellschaft. Aus Sicht der Landwirtschaft führt dies zu einer Reduktion der Arbeitskräfte und einem starken Rückgang des Anteils der landwirtschaftlichen Wertschöpfung am Bruttoinlandprodukt.

Strukturverbesserungen sind Investitionshilfen für die Landwirtschaft. Diese haben zum Ziel, Produktionskosten zu senken, Lebensund Arbeitsverhältnisse in ländlichen Regionen zu verbessern, Strukturen vor Naturereignissen zu schützen sowie ökologische und tierschützerische Ziele zu unterstützen. Die Strukturverbesserungen in der Schweizer Agrarpolitik umfassen zwei Instrumente: Investitionskredite (zinslose Darlehen) und Beiträge (à fonds perdu Zahlungen).

Option für die Ausgestaltung eines agrarpolitischen Instruments. Tailoring bezieht sich darauf, dass eine Massnahme auf den Politikadressaten (d.h. den Landwirt) zugeschnitten ist. Das Zuschneiden kann sich auf verschiedene Charakteristiken beziehen z.B. räumliche (Direktzahlungen für BFF werden nach landwirtschaftlichen Produktionszonen abgestuft) oder zeitliche (ein Zollkontingent kann nur in einem bestimmten Zeitfenster genutzt werden). Durch die Massnahme sollen Mitnahmeeffekte reduziert werden.

Option für die Ausgestaltung eines agrarpolitischen Instruments. Ein agrarpolitisches Instrument soll möglichst zielgenau ausgestaltet werden. Wenn möglich soll dabei auch jedes Instrument nur ein Ziel anvisieren (Tinbergen-Regel). Ein Beispiel für Targeting ist die Ausgestaltung des Schweizer Direktzahlungssystems. Jede der unterschiedlichen Zahlungen ist auf ein spezifisches Ziel ausgerichtet (z.B. Biodiversität, Kulturlandschaft, Versorgungssicherheit etc.). Die Zielausrichtung kann auch im Raum und in der Zeit variiert werden, um die Effektivität der Instrumente zu erhöhen. 
TINBERGENREGEL

TRITTBRETTFAHRER

UMWELTBELASTUNG "OPTIMALE»

VERBÄUERLICHUNG, BÄUERLICH
Die Tinbergen-Regel (benannt nach dem Ökonomen Tinbergen) besagt: «Für jedes Ziel eine Massnahme». Die Regel besagt einerseits, dass man mit einer einzelnen agrarpolitischen Massnahme nicht zwei Ziele gleichzeitig erfüllen kann. Andererseits folgt aus der Tinbergen-Regel, dass für ein bestimmtes Ziel nicht mehr als eine Massnahme nötig wäre. In der Schweizer Agrarpolitik fand die TinbergenRegel Eingang mit dem neuen Direktzahlungssystem in der AP14-17. Die Direktzahlungen sind auf die verschiedenen Verfassungsziele ausgerichtet (Versorgungssicherheit, Kulturlandschaft etc.). Aus einer rationalen Perspektive erschwert die Koppelproduktion in der Landwirtschaft die Anwendung der Tinbergen-Regel in der Agrarpolitik. Konkret stellt sich beispielsweise die Frage, ob es eine Direktzahlung für Biolandbau braucht, wo doch Biodiversität, Landschaftspflege, graslandbasierte Milch- und Fleischproduktion - also konstituierende Elemente des Biolandbaus - bereits über andere Direktzahlungen abgegolten werden.

Allgemein wird als Trittbrettfahren bezeichnet, wenn jemand Nutzen aus Einrichtungen, Handlungen oder Gütern zieht, ohne sich an deren Kosten oder Finanzierung zu beteiligen. Die Problematik entsteht spezifisch im Zusammenhang mit öffentlichen Gütern oder Kollektivgütern. Aus einer rationalen Perspektive wird versucht, agrarpolitische Instrumente so auszugestalten, dass Trittbrettfahren nicht möglich wird. Zudem erklärt das Phänomen des Trittbrettfahrens, weshalb die kollektive Bereitstellung von öffentlichen Gütern schwierig ist.

Begriff aus der Ressourcenökonomie. Die optimale Umweltbelastung liegt dort, wo sich im Preis-Mengen-Diagramm die marginalen Schadenskosten und die marginalen Vermeidungskosten schneiden. Würde man eine Einheit der Umweltbelastung mehr reduzieren, dann wären die Kosten der Vermeidung höher als die Kosten, die durch die Umweltbelastung verursacht werden. Aus einer ökonomischen Perspektive wäre es daher sinnvoller, dieses Geld in die Reduktion anderer Umweltbelastungen zu investieren.

Der Verfassungsartikel 104 zur Landwirtschaft sieht explizit vor, dass bodenbewirtschaftende bäuerliche Betriebe gefördert werden sollen. Dem Begriff bäuerlich kommt daher eine grosse Bedeutung zu. Er definiert, was in der Schweiz verfassungsmässig als förderungswürdig betrachtet werden kann. Es gibt allerdings keine einheitliche Definition, was unter bäuerlich zu verstehen ist. Der Begriff hat sich im Laufe der Zeit immer wieder verändert und ist stark durch gesellschaftliche und politische Debatten geprägt. Von der Verbäuerlichung spricht man im Zusammenhang mit der grundlegenden Struk- 
VERFASSUNG, GESETZ, VERORDNUNG

VOLATILITÄT DER AGRARPREISE

\section{VOLKS-} INITIATIVE, REFERENDA turänderung Ende des 19. Jahrhunderts. Durch die zunehmende Industrialisierung stiegen die Löhne der Arbeiter. Die Landwirtschaft mit ihrer tiefen Wertschöpfung konnte diese Löhne nicht mehr bezahlen. Deshalb beschränkte sich die Arbeit fortan auf Familienmitglieder. Dadurch entstanden die heute prägenden Familienbetriebe in der Schweizer Landwirtschaft.

Die Verfassung ist die oberste Rechtsordnung in der Schweiz und regelt die Form des Staates sowie die Rechte und Pflichten der Bürgerinnen und Bürger. Änderungen der Verfassung müssen in einer Volksabstimmung bestätigt werden. Gesetze sind hierarchisch unter der Verfassung positioniert. Sie werden durch die Legislative erlassen und unterstehen dem fakultativen Referendum. Verordnungen sind die dritte Ebene der Rechtsordnung und werden durch die Exekutive verordnet. Die Grundlage für die Schweizer Agrarpolitik sind die beiden Verfassungsartikel 104 und 104a. Auf der Gesetzesebene sind das Landwirtschaftsgesetz (LwG) sowie das BGBB die zentralen Steuerungsinstrumente. Basierend auf diesen Gesetzen gibt es 49 Verordnungen, welche diese Gesetzgebung konkretisieren. Die Agrarpolitik ist hauptsächlich Bundespolitik. Kantone können aber zusätzliche Regelungen einführen.

Volatilität beschreibt den Schwankungsbereich von Preisen. Preise für landwirtschaftliche Rohstoffe können grossen Schwankungen ausgesetzt sein, da im Verhältnis zur Produktion relativ kleine Mengen gehandelt werden. Tritt ein Angebotsschock ein (z.B. durch Dürre, Schädlinge oder wirtschaftliche Ursachen), dann steigt der Preis schnell an, weil die Nachfrage nach Lebensmitteln eher preisunelastisch ist (Nahrungsmittel braucht man ja immer). Die Preisvolatilität kann die Ernährungssicherheit der Bevölkerung schmälern; insbesondere in Ländern, in denen Nahrungsmittel importiert werden müssen. Die Volatilität der Agrarpreise galt und gilt daher als zentrales Argument für den Schutz und die Förderung der inländischen Nahrungsmittelproduktion sowie die Lagerhaltung von Nahrungsmitteln weltweit.

Referenda und Volksinitiativen sind Instrumente der direkten Demokratie. Die Volksinitiative kann von Stimmberechtigten ergriffen werden und zielt darauf ab, die Verfassungsgrundlage zu ändern. Die Regierung kann einen Gegenentwurf zur Volksinitiative vorschlagen. Das Referendum ist ein Volksentscheid, bei dem die Bürger eine Verfassungsänderung (obligatorisch) oder eine Gesetzesänderung (fakultativ) des Parlamentes bestätigen oder verwerfen können. Die Schweizer Agrarpolitik war lange Zeit durch Referenda charakterisiert, in welchen die Bevölkerung die protektionistische Landwirtschaftspolitik stützte. Ende der 1980er-Jahre trugen Volksinitiativen 
WTO

X-STRUKTUR DER WERTSCHÖPFUNGSKETTE

ZIELE DER AGRARPOLITIK dazu bei, dass die Verfassungsgrundlage in der Schweiz geändert wurde. In den letzten Jahren entstanden immer mehr Volksinitiativen, welche die Schweizer Agrarpolitik herausfordern.

Die World Trade Organization (WTO) mit Sitz in Genf ist eine internationale Organisation, die sich in globalem Kontext mit Handelsund Wirtschaftspolitik befasst. Die WTO wurde im Rahmen der GATT Uruguay-Runde 1995 gegründet und zählt 163 Mitgliedstaaten. Das Ziel der WTO ist der Abbau von Handelshemmnissen und die Förderung des internationalen Freihandels. Die Kernaufgaben der WTO sind die Koordination der Wirtschaftspolitik und die Streitschlichtung zwischen Mitgliedstaaten. Die beiden grundlegenden Regeln innerhalb der WTO sind Meistbegünstigung und Inländerbehandlung. Meistbegünstigung bedeutet, dass die einem Vertragspartner gewährten Handelsvorteile aus Gleichberechtigungsgründen auch gegenüber allen anderen Mitgliedsländern gelten müssen. Inländerbehandlung beschreibt, dass in- und ausländische Waren und Anbieter gleichbehandelt werden müssen.

Die X-Struktur beschreibt eine Wertschöpfungskette, in welcher viele Produzentinnen und Produzenten wenigen Verarbeitern bzw. wenigen Händlern gegenüberstehen, diese aber wiederum viele Konsumentinnen und Konsumenten beliefern. In der Schweiz werden die Nahrungsmittel von vielen Landwirtschaftsbetrieben hergestellt. Sie entsprechen einer atomistischen Struktur. Das hat zur Folge, dass der einzelne Landwirt durch seine Produktionsmenge den Preis für das Produkt nicht bestimmen kann. Auch die Konsumentinnen und Konsumenten weisen eine atomistische Struktur auf. Niemand kann durch seine Nachfrage den Preis für die Nahrungsmittel beeinflussen. Demgegenüber stehen die Abnehmer und Händler. Diese umfassen nur wenige Akteure und ihre Entscheidungen über Mengen können die Preise beeinflussen. Dadurch kann Marktmacht entstehen.

Die Agrarpolitik kennt eine Vielzahl von Zielen. Diese lassen sich grundsätzlich in vier Kategorien einteilen: 1) Produktionsziele, d.h. ein effizienter Einsatz der volkswirtschaftlichen Produktionsfaktoren; 2) Verteilungsziele, d.h. eine gerechte Einkommens- und Vermögensverteilung; 3) Sicherungsziele, d.h. eine stabile Wirtschaft und Versorgungssicherheit; 4) wirtschaftliche Freiheit. Diese Ziele lassen sich nicht immer widerspruchslos miteinander verbinden. So kann beispielsweise das Ziel der Versorgungssicherheit mit Grundsätzen der wirtschaftlichen Freiheit in Konflikt stehen. Auch ein effizienter Einsatz der Produktionsfaktoren und die Stabilität von Systemen können nicht immer gleichzeitig erreicht werden. Diese Konflikte werden als Trade-offs bezeichnet und es bedarf politisch einer 
ZOLL-

KONTIN-

GENT

Güterabwägung zwischen den Zielen. Zielkonflikte sind ein wichtiges Charakteristikum der Agrarpolitik. Exemplarisch steht dabei der Konflikt zwischen einem hohen Selbstversorgungsgrad und einer möglichst umweltverträglichen Produktion. Wichtig ist auch festzuhalten, dass sich die agrarpolitischen Ziele gesellschaftlichen und wirtschaftlichen Veränderungen über die Zeit anpassen.

Ein Zollkontingent ist ein zweistufiges Zollsystem, bei dem eine definierte Kontingentsmenge zu einem tiefen Zollansatz importiert werden darf. Ausserhalb dieses Kontingents gilt ein sehr hoher Ausserkontingentszollansatz, der weitere Importe praktisch verhindert. Der Grenzschutz von Schweizer Agrarprodukten erfolgt zum allergrössten Teil über diese Zollkontingente. Sie garantieren einen effektiven Schutz der inländischen Produktion vor billigeren Importen. 


\section{Referenzen}

Abdulai, A., 2002. Using threshold cointegration to estimate asymmetric price transmission in the Swiss pork market. Applied Economics 34, 679-687, Taylor \& Francis.

Abler, D., 2004. Multifunctionality, Agricultural Policy, and Environmental Policy. Agriculture and Resource Economics Review 33, 8-18, Cambridge University Press.

Albrecht, M., Schmid, B., Obrist, M.K., Schüpbach, B., Kleijn, D., Duelli, P., 2010. Effects of ecological compensation meadows on arthropod diversity in adjacent intensively managed grassland. Biological Conservation 143, 642-649, Elsevier.

Alexander, P., Brown, C., Arneth, A., Finnigan, J., Moran, D., Rounsevell, M.D.A., 2017. Losses, inefficiencies and waste in the global food system. Agricultural Systems 153, 190-200, Elsevier.

Alig, M., Nathani, C., Flury, C., 2019. Umweltauswirkungen einer Marktöffnung im Landwirtschaftsbereich - Analyse drei theoretischer Handelsszenarien.

Schlussbericht im Auftrag des Bundesamtes für Umwelt

Alston, J.M., James, J.S., 2002. The incidence of agricultural policy. Handbook of Agricultural Economics 2, 1689-1749, Elsevier.

Alston, J.M., Pardey, P.G., 2014. Agriculture in the Global Economy. Journal of Economic Perspectives 28, 121-146, American Economic Association.

AMIS, 2020. Agricultural Market Information System (AMIS) In: (FAO), F.a.A.O.o.t.U.N. (Ed.), AMIS Market Monitor.

Anderegg, R., 1999. Grundzüge der Agrarpolitik. Walter de Gruyter.

Anderson, K., 2000. Agriculture's multifunctionality and the WTO. Australian Journal of Agricultural and Resource Economics 44, 475-494, Wiley-Blackwell.

Anderson, K., Martin, W.A., 2005. Agricultural Trade Reform and the Doha Development Agenda. The World Economy 28, 1301-1327, John Wiley \& Sons.

Antle, J.M., Ray, S., 2020. Sustainable Agricultural Development. Palgrave Studies in Agricultural Economics and Food Policy.

Aouinaït, C., Christen, D., Zbinden, K.K., 2020. Chapter 11 - Traditional food, legal and regulatory issues in Switzerland. In: Braun, S., Zübert, C., Argyropoulos, D., Casado Hebrard, F.J. (Eds.), Nutritional and Health Aspects of Food in Western Europe, 219-238, Academic Press.

Auderset, J., Moser, P., 2018. Die Agrarfrage in der Industriegesellschaft:

Wissenskultur, Machtverhältnisse und natürliche Ressourcen in der agrarisch- 
industriellen Wissensgesellschaft (1850-1950). Agricultural History 93, 191-193, Böhlau Verlag.

Aviron, S., Nitsch, H., Jeanneret, P., Buholzer, S., Luka, H., Pfiffner, L., Pozzi, S., Schüpbach, B., Walter, T., Herzog, F., 2008. Ecological cross compliance promotes farmland biodiversity in Switzerland. Frontiers in Ecology and the Environment 7, 247-252, The Ecological Society of America.

Bartkowski, B., Droste, N., Ließ, M., Sidemo-Holm, W., Weller, U., Brady, M.V., 2021. Payments by modelled results: A novel design for agri-environmental schemes. Land Use Policy 102, 105230, Elsevier.

Basso, B., Antle, J., 2020. Digital agriculture to design sustainable agricultural systems. Nature Sustainability 3, 254-256, Nature Publishing Group.

Baumol, W.J., Oates, W.E., 1988. The theory of environmental policy. 2nd edn. Cambridge University Press.

Binder, S., Mann, S., 2019. Wie fördern die Kantone ihre Landwirtschaft? Agrarforschung Schweiz 10, 316-321, Agroscope.

Binswanger, M., 2020. Globalisierung und Landwirtschaft. Mehr Wohlstand durch weniger Freihandel. Picus Verlag.

Birrer, S., Spiess, M., Herzog, F., Jenny, M., Kohli, L., Lugrin, B., 2007. The Swiss agrienvironment scheme promotes farmland birds: but only moderately. Journal of Ornithology 148, 295-303, Springer.

Blandford, D., Tangerman, S., 2021. Current Issues in Global Agricultural and Trade Policy. World Scientific.

Bokusheva, R., Grau, A., Fischer, S., Grass, M., 2019. Spielt der Wettbewerb im Schweizer Food-Markt? Die Volkswirtschaft 3, 25-28, Staatssekretariat für Wirtschaft SECO.

Bowen, H.P., Leamer, E.E., Sveikauskas, L., 1987. Multicountry, Multifactor Tests of the Factor Abundance Theory. The American Economic Review 77, 791-809, American Economic Association.

Bromley, D., 2000. Can Agriculture Become an Environmental Asset? World Economics 1, 127-139, John Wiley \& Sons.

Bromley, D.W., Hodge, I., 1990. Private property rights and presumptive policy entitlements: reconsidering the premises of rural policy. European Review of Agricultural Economics 17, 197-214, Walter de Gruyter.

Bureau, J.-C., Guimbard, H., Jean, S., 2019. Agricultural Trade Liberalisation in the 21st Century: Has It Done the Business? Journal of Agricultural Economics 70, 3-25, Wiley-Blackwell. 
Burton, R.J., Forney, J., Stock, P., Sutherland, L.-A., 2021. The good farmer: Culture and identity in food and agriculture. Routledge.

Burton, R.J.F., Schwarz, G., 2013. Result-oriented agri-environmental schemes in Europe and their potential for promoting behavioural change. Land Use Policy 30, 628-641, Elsevier.

Bystricky, M., Nemecek, T., Krause, S., Gaillard, G., 2020. Potenzielle Umweltfolgen einer Umsetzung der Trinkwasserinitiative. Agroscope Science 99, Agroscope.

Chabé-Ferret, S., Le Coent, P., Reynaud, A., Subervie, J., Lepercq, D., 2019. Can we nudge farmers into saving water? Evidence from a randomised experiment. European Review of Agricultural Economics 46, 393-416, Oxford University Press.

Chambers, R.G., 2002. Information, incentives, and the design of agricultural policies. Handbook of Agricultural Economics 2B, 1751-1825, Elsevier.

Chambers, R.G., Voica, D.C., 2017. «Decoupled» Farm Program Payments are Really Decoupled: The Theory. American Journal of Agricultural Economics 99, 773-782, Wiley-Blackwell.

Cochrane, W.W., 1958. Farm Prices: Myth and Reality. Minnesota Archive Editions, University of Minnesota.

Collier, P., 2011. Der hungrige Planet: wie können wir Wohlstand mehren, ohne die Erde auszuplündern. Siedler Verlag.

Costinot, A., Donaldson, D., 2012. Ricardo's Theory of Comparative Advantage: Old Idea, New Evidence. American Economic Review 102, 453-458, American Economic Association.

Costinot, A., Donaldson, D., Smith, C., 2016. Evolving Comparative Advantage and the Impact of Climate Change in Agricultural Markets: Evidence from 1.7 Million Fields around the World. Journal of Political Economy 124, 205-248, University of Chicago Press.

Costinot, A., Vogel, J., 2015. Beyond Ricardo: Assignment Models in International Trade. Annual Review of Economics 7, 31-62, Annual Reviews.

Dabbert, S., Braun, J., 2012. Landwirtschaftliche Betriebslehre. UTB.

Dasgupta, P., 2021. The Economics of Biodiversity: The Dasgupta Review. HM Treasury.

De Gorter, H., Swinnen, J., 2002. Political economy of agricultural policy. In: Gardner, B.L., Rausser, G.C. (Eds.), Handbook of Agricultural Economics 2B, Chapter 36, 1893-1943, Elsevier

De Schutter, O., 2011. How not to think of land-grabbing: three critiques of largescale investments in farmland. The Journal of Peasant Studies 38, 249-279, Taylor \& Francis. 
De Schutter, O., 2017. The political economy of food systems reform. European Review of Agricultural Economics 44, 705-731, Oxford University Press.

De Schutter, O., Jacobs, N., Clément, C., 2020. A «Common Food Policy» for Europe: How governance reforms can spark a shift to healthy diets and sustainable food systems. Food Policy 96, 101849, Elsevier.

Deconinck, K., 2020. Concentration in Seed and Biotech Markets: Extent, Causes, and Impacts. Annual Review of Resource Economics 12, 129-147, Annual Reviews.

Deininger, K., Feder, G., 2009. Land Registration, Governance, and Development: Evidence and Implications for Policy. The World Bank Research Observer 24, 233 266, Oxford University Press.

Desta, M.G., 2016. Trade in Agricultural Products: Should Developing Countries Give Up on the WTO Promise for a Fair and Market-Oriented Agricultural Trading System? A Historical and Theoretical Analysis. In: Bungenberg, M., Herrmann, C., Krajewski, M., Terhechte, J.P. (Eds.), European Yearbook of International Economic Law 2016, 67-102, Springer.

Diamond, J., 2014. Kollaps: Warum Gesellschaften überleben oder untergehen. S. Fischer Verlag.

Downs, A., 1957. An Economic Theroy of Political Action in a Democracy. The Journal of Political Economy 65, 135-150, University of Chicago Press.

Dümmler, P., Roten, N., 2018. Eine Agrarpolitik mit Zukunft. Eine Zehn-PunkteStrategie für Konsumenten, Steuerzahler und landwirtschaftliche Unternehmer. avenir debatte, Avenir Suisse.

Eastwood, R., Lipton, M., Newell, A., 2010. Farm Size. Handbook of Agricultural Economics 4, Chapter 65, 3323-3397, Elsevier.

Ecoplan, HAFL, 2016. Beitrag der Landwirtschaft und der Agrarpolitik zur Vitalität und Attraktivität des ländlichen Raums. Studie im Auftrag des Bundesamts für Landwirtschaft (BLW).

EFK, 2015. Investitionshilfen in der Landwirtschaft Evaluation der Konzeption, Kosten und Wirksamkeit. Eidgenössische Finanzkontrolle.

Ehlers, M.-H., Huber, R., Finger, R., 2021. Agricultural Policy in the Era of Digitalisation. Food Policy 100, 102019, Elsevier.

Ehlers, M.-H., Finger, R., El Benni, N., Gocht, A., Sørensen, C.A.G., Gusset, M., Pfeifer, C., Poppe, K., Regan, A., Rose, D.C., Wolfert, S., Huber, R. 2022 Scenarios for European agricultural policymaking in the era of digitalisation. Agricultural Systems. Elsevier.

El Benni, N., Finger, R., Mann, S., 2012. Effects of agricultural policy reforms and farm characteristics on income risk in Swiss agriculture. Agricultural Finance Review 72, 301-324, Emerald Group Publishing. 
Engel, S., 2016. The devil in the detail: a practical guide on designing payments for environmental services. International Review of Environmental and Resource Economics 9, 131-177, Now Publishers.

Engel, S., Pagiola, S., Wunder, S., 2008. Designing payments for environmental services in theory and practice: An overview of the issues. Ecological Economics 65, 663-674, Elsevier.

Eory, V., Pellerin, S., Carmona Garcia, G., Lehtonen, H., Licite, I., Mattila, H., LundSørensen, T., Muldowney, J., Popluga, D., Strandmark, L., Schulte, R., 2018. Marginal abatement cost curves for agricultural climate policy: State-of-the art, lessons learnt and future potential. Journal of Cleaner Production 182, 705-716, Elsevier.

Erjavec, E., Lovec, M., 2017. Research of European Union's Common Agricultural Policy: disciplinary boundaries and beyond. European Review of Agricultural Economics 44, 732-754, Oxford University Press.

Eş, I., Gavahian, M., Marti-Quijal, F.J., Lorenzo, J.M., Mousavi Khaneghah, A., Tsatsanis, C., Kampranis, S.C., Barba, F.J., 2019. The application of the CRISPR-Cas9 genome editing machinery in food and agricultural science: Current status, future perspectives, and associated challenges. Biotechnology Advances 37, 410-421, Elsevier.

Esposti, R., Listorti, G., 2018. Price Transmission in the Swiss Wheat Market: Does Sophisticated Border Protection Make the Difference? The International Trade Journal 32, 209-238, Taylor \& Francis.

Eyhorn, F., Muller, A., Reganold, J.P., Frison, E., Herren, H.R., Luttikholt, L., Mueller, A., Sanders, J., Scialabba, N.E.-H., Seufert, V., Smith, P., 2019. Sustainability in global agriculture driven by organic farming. Nature Sustainability 2, 253-255, Nature Publishing Group.

FAO, 2011. The State of Food Insecurity in the World. How does international price volatility affect domestic economies and food security? Food and Agriculture Organization of the United Nations.

Feindt, P.H., Krämer, C., Früh-Müller, A., Heißenhuber, A., Pahl-Wostl, C., Purnhagen, K.P., Thomas, F., van Bers, C., Wolters, V., 2019. Ein neuer Gesellschaftsvertrag für eine nachhaltige Landwirtschaft : Wege zu einer integrativen Politik für den Agrarsektor. Springer Nature.

Ferraro, P.J., 2008. Asymmetric information and contract design for payments for environmental services. Ecological Economics 65, 810-821, Elsevier.

Fesenfeld, L.P., Wicki, M., Sun, Y., Bernauer, T., 2020. Policy packaging can make food system transformation feasible. Nature Food 1, 173-182, Springer.

Finger, R., 2021. No pesticide-free Switzerland. Nature Plants 7, 1324-1325. 
Finger, R., El Benni, N., 2014. A Note on the Effects of the Income Stabilisation Tool on Income Inequality in Agriculture. Journal of Agricultural Economics 65, 739-745, Wiley-Blackwell.

Finger, R., Lehmann, N., 2012. The influence of direct payments on farmers' hail insurance decisions. Agricultural Economics 43, 343-354, John Wiley \& Sons.

Finger, R., Listorti, G., Tonini, A., 2017. The Swiss payment for milk processed into cheese: ex post and ex ante analysis. Agricultural Economics 48, 437-448, John Wiley \& Sons.

Fisher, B., Turner, K., Zylstra, M., Brouwer, R., Groot, R., Farber, S., Ferraro, P., Green, R., Hadley, D., Harlow, J., Jefferiss, P., Kirkby, C., Morling, P., Mowatt, S., Naidoo, R., Paavola, J., Strassburg, B., Yu, D., Balmford, A., 2008. Ecosystem services and economic theory: integration for policy-relevant research. Ecological Applications 18, 2050-2067, Ecological Society of America.

Flury, C., Abegg, C., Jeannerat, H., 2017. Zwischenevaluation «Projekte zur regionalen Entwicklung». Bericht zuhanden des Bundesamts für Landwirtschaft.

Flury, C., Gerber, A., Giuliani, G., Berger, S., 2012. Evaluation der wirtschaftlichen Bedeutung und Erfolgsfaktoren regionaler Verarbeitungsbetriebe unter Berücksichtigung der Investitionshilfen. Bericht zuhanden des Bundesamts für Landwirtschaft, Flury\&Giuliani.

Flury, C., Huber, R., 2007. Evaluation der Jointness in der Schweizer Landwirtschaft. Agrarforschung 14, 572-574, Recherche agronomique suisse.

Flury, C., Peter, K., 2011. Evaluation der Effektivität von Betriebshilfemassnahmen im Zusammenhang mit finanzieller Bedrängnis und Verschuldung. Bericht zuhanden des Bundesamts für Landwirtschaft, Flury\&Giuliani.

Fontana, M.-C., Haering, B., Koch, P., Meier, B., 2019. Evaluation der Biodiversitätsbeiträge. Bericht zuhanden des Bundesamts für Landwirtschaft.

Fraser, R., 2009. Land Heterogeneity, Agricultural Income Forgone and Environmental Benefit: An Assessment of Incentive Compatibility Problems in Environmental Stewardship Schemes. Journal of Agricultural Economics 60, 190201, Wiley-Blackwell.

Frey, B.S., 1985. Internationale Politische Ökonomie. Vahlen.

Friel, S., Schram, A., Townsend, B., 2020. The nexus between international trade, food systems, malnutrition and climate change. Nature Food 1, 51-58, Springer.

Friis, C., Nielsen, J.Ø., 2018. Telecoupling. Exploring Land-Use Change in a Globalized World. Palgrave Studies in Natural Resource Management.

Fritzsche, B., Lemmenmeier, M., 1994. Die revolutionäre Umgestaltung von Wirtschaft, Gesellschaft und Staat 1780-1870. Geschichte des Kantons Zürich. 
Gardner, B.L., 1992. Changing economic perspectives on the farm problem. Journal of economic literature 30, 62-101, American Economic Association.

Gawith, D., Hodge, I., 2019. Focus rural land policies on ecosystem services, not agriculture. Nature Ecology \& Evolution 3, 1136-1139, Nature Publishing Group.

Gentile, M., Loi, A., Gentile, E., 2019. Warum sind Dünger und Pestizide in der Schweiz teurer als in der EU? Die Volkswirtschaft 3, 13-15, Staatssekretariat für Wirtschaft SECO.

Gillis, M., Perkins, D.H., Roemer, M., Snodgrass, D.R., 1992. Economics of development. WW Norton.

Godfray, H.C.J., Beddington, J.R., Crute, I.R., Haddad, L., Lawrence, D., Muir, J.F., Pretty, J., Robinson, S., Thomas, S.M., Toulmin, C., 2010. Food Security: The Challenge of Feeding 9 Billion People. Science 327, 812-818, American Association for the Advancement of Science.

Goetz, R.-U., Schmid, H., Lehmann, B., 2006. Determining the economic gains from regulation at the extensive and intensive margins. European Review of Agricultural Economics 33, 1-30, Oxford University Press.

Golan, E., Kuchler, F., Mitchell, L., Greene, C., Jessup, A., 2001. Economics of Food Labeling. Journal of Consumer Policy 24, 117-184, Springer.

Graeub, B.E., Chappell, M.J., Wittman, H., Ledermann, S., Kerr, R.B., GemmillHerren, B., 2016. The State of Family Farms in the World. World Development 87, 1-15, Elsevier.

Grass, I., Batáry, P., Tscharntke, T., 2020. Combining land-sparing and land-sharing in European landscapes. Advances in Ecological Research. Academic Press.

Graubner, M., 2017. Lost in space? The effect of direct payments on land rental prices. European Review of Agricultural Economics 45, 143-171, Oxford University Press.

Gray, E., Adenäuer, L., Flaig, D., van Tongeren, F., 2017. Evaluation of the relevance of border protection for agriculture in Switzerland. OECD Food, Agriculture and Fisheries Papers 109, OECD Publising.

Grêt-Regamey, A., Huber, S.H., Huber, R., 2019. Actors' diversity and the resilience of social-ecological systems to global change. Nature Sustainability 2, 290-297, Nature Publishing Group.

Groher, T., Heitkämper, K., Walter, A., Liebisch, F., Umstätter, C., 2020. Status quo of adoption of precision agriculture enabling technologies in Swiss plant production. Precision Agriculture21, 1327-1350, Springer.

Haberl, H., Erb, K.H., Krausmann, F., Gaube, V., Bondeau, A., Plutzar, C., Gingrich, S., Lucht, W., Fischer-Kowalski, M., 2007. Quantifying and mapping the human appropriation of net primary production in earth's terrestrial ecosystems. 
Proceedings of the National Academy of Science 104, 12942-12947, National Academy of Science.

Haller, T., 2011. Bedeutung der Landwirtschaft in einer zunehmend urbanen Gesellschaft. ETH Zurich.

Hanley, N., Banerjee, S., Lennox, G.D., Armsworth, P.R., 2012. How should we incentivize private landowners to «produce» more biodiversity? Oxford Review of Economic Policy 28, 93-113, Oxford University Press.

Harris, J.M., Roach, B., 2013. Environmental and natural resource economics: A contemporary approach. ME Sharpe.

Harvey, D.R., 2003. Agri-environmental Relationships and Multifunctionality: Further Considerations. The World Economy 26, 705-725, John Wiley \& Sons.

Hasegawa, T., Havlík, P., Frank, S., Palazzo, A., Valin, H., 2019. Tackling food consumption inequality to fight hunger without pressuring the environment. Nature Sustainability 2, 826-833, Nature Publishing Group.

Heal, G.M., Small, A.A., 2002. Agriculture and Ecosystem Services. In: Gardner, B.L., Rausser, G.C. (Eds.), Handbook of Agricultural Economics 2: Agriculture and its external linkages, 1341-1369, Elsevier.

Hediger, W., Knickel, K., 2009. Multifunctionality and Sustainability of Agriculture and Rural Areas: A Welfare Economics Perspective. Journal of Environmental Policy \& Planning 11, 291-313, Taylor \& Francis.

Hendrikse, G., 2003. Economics and management of organizations: co-ordination, motivation and strategy. McGraw-Hill.

Henrichsmeyer, W., Witzke, H.P., 1994. Agrarpolitik Band 2: Bewertung und Willensbildung. Ulmer.

Herzog, F., Dreier, S., Hofer, G., Marfurt, C., Schüpbach, B., Spiess, M., Walter, T., 2005. Effect of ecological compensation areas on floristic and breeding bird diversity in Swiss agricultural landscapes. Agriculture, Ecosystems \& Environment 108, 189-204, Elsevier.

Herzog, F., Jacot, K., Tschumi, M., Walter, T., 2017. The Role of Pest Management in Driving Agri-environment Schemes in Switzerland. Environmental Pest Management, 385-403, John Wiley \& Sons.

Herzog, F., Prasuhn, V., Spiess, E., Richner, W., 2008. Environmental crosscompliance mitigates nitrogen and phosphorus pollution from Swiss agriculture. Environmental Science \& Policy 11, 655-668, Elsevier.

Hickel, J., 2016. The true extent of global poverty and hunger: questioning the good news narrative of the Millennium Development Goals. Third World Quarterly 37, 749-767, Taylor \& Francis. 
Hillen, J., 2019. Market Integration and Market Efficiency under Seasonal Tariff Rate Quotas. Journal of Agricultural Economics 70, 859-873, Wiley-Blackwell.

Hillen, J., 2021. Vertical price transmission in Swiss dairy and cheese value chains. Agricultural and Food Economics 9, 13, Springer.

Hillen, J., von Cramon-Taubadel, S., 2019. Protecting the Swiss milk market from foreign price shocks: Public border protection vs. quality differentiation. Agribusiness 35, 516-536, John Wiley \& Sons.

Hirsch, S., 2018. Successful in the long run: A meta-regression analysis of persistent firm profits. Journal of Economic Surveys 32, 23-49, John Wiley \& Sons.

Hirschi, C., Widmer, A., Briner, S., Huber, R., 2013. Combining Policy Network and Model-Based Scenario Analyses: An Assessment of Future Ecosystem Goods and Services in Swiss Mountain Regions. Ecology and Society 18, The Resilience Alliance.

Höchli, C., Schläpfer, F., Engel, S., 2009. Eignung von Ausschreibeverfahren zur Förderung gemeinwirtschaftlicher Leistungen in der Schweizer Landwirtschaft. Bundesamt für Landwirtschaft, ETH Zürich.

Hodge, I., 2002. Agri-environmental Pelationships and the Choice of Policy Mechanism. The World Economy 23, 257-273, John Wiley \& Sons.

Hovhannisyan, V., Cho, C., Bozic, M., 2018. The relationship between price and retail concentration: evidence from the US food industry. European Review of Agricultural Economics 46, 319-345, Oxford University Press.

Huber, R., 2010. Jointness between agriculture and environmental goods and services: An economic analysis in the Swiss lowlands. ETH Zürich.

Huber, R., Finger, R., 2019. Popular initiatives increasingly stimulate agricultural policy in Switzerland. EuroChoices 18, 38-39, Wiley-Blackwell.

Huber, R., Flury, C., Meier, B., Mack, G., 2017a. Direktzahlungen sorgfältig aufeinander abstimmen. Agrarforschung Schweiz 8, 26-29, Agroscope.

Huber, R., Häberli, C., 2010. A «beyond WTO» scenario for Swiss agriculture: Consequences for income generation and the provision of public goods. Yearbook of Socioeconomics in Agriculture 2010, 361-400, Swiss Society for Agricultural Economics and Rural Sociologie.

Huber, R., Lehmann, B., 2010. Economies of scope in the agricultural provision of ecosystem services: An application to a high cost production region. German Journal of Agriculture Economics (GJAE) 59, 91-105, Deutscher Fachverlag.

Huber, R., Snell, R., Monin, F., Brunner, S.H., Schmatz, D., Finger, R., 2017b. Interaction effects of targeted agri-environmental payments on non-marketed goods and services under climate change in a mountain region. Land Use Policy 66, 49-60, Elsevier. 
IFPRI, 2019. Global Food Policy Report. International Food Policy Research Institute.

Ingold, K.M., Lieberherr, E., Schläpfer, I., Steinmann, K., Zimmermann, W., 2016. Umweltpolitik der Schweiz - ein Lehrbuch. Dike Verlag.

Jack, B.K., Kousky, C., Sims, K.R.E., 2008. Designing payments for ecosystem services: Lessons from previous experience with incentive-based mechanisms. Proceedings of the National Academy of Sciences 105, 9465-9470, National Academy of Science.

Janker, J., Mann, S., 2020. Understanding the social dimension of sustainability in agriculture: a critical review of sustainability assessment tools. Environment, Development and Sustainability 22, 1671-1691, Springer.

Janssens, C., Havlík, P., Krisztin, T., Baker, J., Frank, S., Hasegawa, T., Leclère, D., Ohrel, S., Ragnauth, S., Schmid, E., Valin, H., Van Lipzig, N., Maertens, M., 2020. Global hunger and climate change adaptation through international trade. Nature Climate Change 10, 829-835, Nature Publishing Group.

Jenny, M., Studer, J., Bosshard, A., 2018. Evaluation Vernetzungsprojekte. Schweizerische Vogelwarte, Sempach.

Jörin, R., 2001. Die Instrumente des Marktzutritts in der WTO: Erfahrungen und Folgerungen der Schweiz. Schriftenreihe/ETH Zürich, Institut für Agrarwirtschaft.

Kampmann, D., Herzog, F., Jeanneret, P., Konold, W., Peter, M., Walter, T., Wildi, O., Lüscher, A., 2008. Mountain grassland biodiversity: Impact of site conditions versus management type. Journal for Nature Conservation 16, 12-25, Elsevier.

Kampmann, D., Lüscher, A., Konold, W., Herzog, F., 2012. Agri-environment scheme protects diversity of mountain grassland species. Land Use Policy 29, 569-576, Elsevier.

Kanter, D.R., Bartolini, F., Kugelberg, S., Leip, A., Oenema, O., Uwizeye, A., 2020a. Nitrogen pollution policy beyond the farm. Nature Food 1, 27-32; Springer.

Kanter, D.R., Chodos, O., Nordland, O., Rutigliano, M., Winiwarter, W., 2020b. Gaps and opportunities in nitrogen pollution policies around the world. Nature Sustainability 3, 956-963, Nature Publishing Group.

Kazukauskas, A., Newman, C., Clancy, D., Sauer, J., 2013. Disinvestment, Farm Size, and Gradual Farm Exit: The Impact of Subsidy Decoupling in a European Context. American Journal of Agricultural Economics 95, 1068-1087, Wiley-Blackwell.

Khush, G.S., 2001. Green revolution: the way forward. Nature Reviews Genetics 2, 815-822, Nature Publishing Group.

Kinnunen, P., Guillaume, J.H.A., Taka, M., D’Odorico, P., Siebert, S., Puma, M.J., Jalava, M., Kummu, M., 2020. Local food crop production can fulfil demand for less than one-third of the population. Nature Food 1, 229-237, Springer. 
Kirchgässner, G., 2010. Homo oeconomicus: The economic model of behaviour and its applications in economics and other social sciences. Springer.

Knop, E.V.A., Kleijn, D., Herzog, F., Schmid, B., 2006. Effectiveness of the Swiss agrienvironment scheme in promoting biodiversity. Journal of Applied Ecology 43, 120127, Wiley-Blackwell.

Kohler, A., 2016. Die Auswirkungen des Käsefreihandels mit der EU. Ökonomie Agroscope Science Nr. 39, Agroscope.

Koning, N., Pinstrup-Andersen, P., 2007. Agricultural trade liberalization and the least developed countries. Springer.

Krausmann, F., Langthaler, E., 2019. Food regimes and their trade links: A socioecological perspective. Ecological Economics 160, 87-95, Elsevier.

Kreft, C., Huber, R., Wuepper, D., Finger, R., 2021. The role of non-cognitive skills in farmers' adoption of climate change mitigation measures. Ecological Economics 189, 107169, Elsevier.

Krugman, P., 2009. The Increasing Returns Revolution in Trade and Geography. American Economic Review 99, 561-571, American Economic Association.

Kuhfuss, L., Préget, R., Thoyer, S., Hanley, N., 2016a. Nudging farmers to enrol land into agri-environmental schemes: the role of a collective bonus. European Review of Agricultural Economics 43, 609-636, Oxford University Press.

Kuhfuss, L., Préget, R., Thoyer, S., Hanley, N., Coent, P.L., Désolé, M., $2016 \mathrm{~b}$. Nudges, Social Norms, and Permanence in Agri-environmental Schemes. Land Economics 92, 641-655, University of Wisconsin Press.

Kupper, T., Bonjour, C., Menzi, H., 2015. Evolution of farm and manure management and their influence on ammonia emissions from agriculture in Switzerland between 1990 and 2010. Atmospheric Environment 103, 215-221, Elsevier.

Lambin, E.F., Gibbs, H.K., Heilmayr, R., Carlson, K.M., Fleck, L.C., Garrett, R.D., le Polain de Waroux, Y., McDermott, C.L., McLaughlin, D., Newton, P., Nolte, C., Pacheco, P., Rausch, L.L., Streck, C., Thorlakson, T., Walker, N.F., 2018. The role of supply-chain initiatives in reducing deforestation. Nature Climate Change 8, 109116, Nature Publishing Group.

Lawry, S., Samii, C., Hall, R., Leopold, A., Hornby, D., Mtero, F., 2014. The Impact of Land Property Rights Interventions on Investment and Agricultural Productivity in Developing Countries: a Systematic Review. Campbell Systematic Reviews 10, 1104, John Wiley \& Sons.

Leclère, D., Obersteiner, M., Barrett, M., Butchart, S.H.M., Chaudhary, A., De Palma, A., DeClerck, F.A.J., Di Marco, M., Doelman, J.C., Dürauer, M., Freeman, R., Harfoot, M., Hasegawa, T., Hellweg, S., Hilbers, J.P., Hill, S.L.L., Humpenöder, F., Jennings, N., 
Krisztin, T., Mace, G.M., Ohashi, H., Popp, A., Purvis, A., Schipper, A.M., Tabeau, A., Valin, H., van Meijl, H., van Zeist, W.-J., Visconti, P., Alkemade, R., Almond, R., Bunting, G., Burgess, N.D., Cornell, S.E., Di Fulvio, F., Ferrier, S., Fritz, S., Fujimori, S., Grooten, M., Harwood, T., Havlík, P., Herrero, M., Hoskins, A.J., Jung, M., Kram, T., Lotze-Campen, H., Matsui, T., Meyer, C., Nel, D., Newbold, T., Schmidt-Traub, G., Stehfest, E., Strassburg, B.B.N., van Vuuren, D.P., Ware, C., Watson, J.E.M., Wu, W., Young, L., 2020. Bending the curve of terrestrial biodiversity needs an integrated strategy. Nature 585, 551-556, Macmillan Publishers.

Leifeld, J., Fuhrer, J., 2005. Greenhouse gas emissions from Swiss agriculture since 1990: implications for environmental policies to mitigate global warming.

Environmental Science \& Policy 8, 410-417, Elsevier.

Levins, R.A., Cochrane, W.W., 1996. The Treadmill Revisited. Land Economics 72, 550-553, University of Wisconsin Press.

Lichtenberg, E., 2002. Agriculture and the environment. In: Gardner, B.L., Rausser, G.C. (Eds.), Handbook of agricultural economics 2, Chapter 23, 1250-1296, Elsevier.

Loft, L., Gehrig, S., Salk, C., Rommel, J., 2020. Fair payments for effective environmental conservation. Proceedings of the National Academy of Sciences 117, 14094-14101, National Academy of Sciences.

Loi, A., Esposti, R., Gentile, M., Bruni, M., Saguatti, A., Berisio, S., Cuppari, L., Aragrande, M., Haller, T., Huber, M., 2016. Policy evaluation of tariff rate quotas. Report mandated by the Swiss Federal Office of Agriculture, Areté srl.

Lowder, S.K., Skoet, J., Raney, T., 2016. The Number, Size, and Distribution of Farms, Smallholder Farms, and Family Farms Worldwide. World Development 87, 16-29, Elsevier.

Lundberg, L., Persson, U.M., Alpizar, F., Lindgren, K., 2018. Context Matters: Exploring the Cost-effectiveness of Fixed Payments and Procurement Auctions for PES. Ecological Economics 146, 347-358, Elsevier.

Lusk, J.L., 2013. Lunch with Pigou: Externalities and the «Hidden» Cost of Food. Agricultural and Resource Economics Review 42, 419-435, Cambridge University Press.

Mack, G., Huber, R., 2017. On-farm compliance costs and N surplus reduction of mixed dairy farms under grassland-based feeding systems. Agricultural Systems 154, 34-44, Elsevier.

Mack, G., Möhring, A., Kohler, A., Wunderlich, A., 2017. Modellprojektionen mit SWISSland und CAPRI zu den Auswirkungen einer Marktöffnung für die Schweizer Landwirtschaft. Ökonomie Agroscope Science, Nr. 56, Agroscope.

Mack, G., Ritzel, C., Heitkämper, K., El Benni, N., 2021. The Effect of Administrative Burden on Farmers' Perceptions of Cross-Compliance-Based Direct Payment Policy. Public Administration Review 81, 664-675, Wiley-Blackwell. 
Mack, G., Ritzel, C., Jan, P., 2020. Determinants for the Implementation of Action-, Result- and Multi-Actor-Oriented Agri-Environment Schemes in Switzerland. Ecological Economics 176, 106715, Elsevier.

Mankiw, N.G., Romer, D., Weil, D.N., 1992. A Contribution to the Empirics of Economic Growth. The Quarterly Journal of Economics 107, 407-437, Oxford University Press.

Mann, S., 2003. Doing it the Swiss Way. EuroChoices 2, 32-35, Wiley-Blackwell.

Mann, S., 2006a. Das verhängnisvolle Paradigma staatlicher Kostendeckung in der Landwirtschaft. Agrarwirtschaft und Agrarsoziologie 06, 1-15, Gesellschaft für Agrarwirtschaft undAgrarsoziologie.

Mann, S., 2006b. Merit goods in a utilitarian framework. Review of Political Economy 18, 509-520, Taylor \& Francis.

Mann, S., Lanz, S., 2013. Happy Tinbergen: Switzerland's New Direct Payment System. EuroChoices 12, 24-28, Wiley-Blackwell.

Mann, S., Logatcheva, K., van Galen, M., Rau, M.-L., 2019. Brot, Joghurt und Schinken: Der Mehrpreis steckt im Detailhandel. Die Volkswirtschaft 3, 20-24, Staatssekretariat für Wirtschaft SECO.

Mann, S., Mack, G., 2004. Wirkungsanalyse der Allgemeinen Direktzahlungen. FATSchriftenreihe Nr. 64. Agroscope.

Manning, P., van der Plas, F., Soliveres, S., Allan, E., Maestre, F.T., Mace, G., Whittingham, M.J., Fischer, M., 2018. Redefining ecosystem multifunctionality. Nature Ecology \& Evolution 2, 427-436.

Martin, W., 2018. A Research Agenda for International Agricultural Trade. Applied Economic Perspectives and Policy 40, 155-173, Oxford University Press.

Matthews, A., 2013. Greening agricultural payments in the EU's Common Agricultural Policy. Bio-based and Applied Economics 2, 1-27, Firenze University Press.

MEA, 2005. Millennium Ecosystem Assessment. Ecosystems and human well-being. Island press.

Mérel, P., Sexton, R.J., 2017. Buyer power with atomistic upstream entry: Can downstream consolidation increase production and welfare? International Journal of Industrial Organization 50, 259-293, Elsevier.

Mettepenningen, E., Beckmann, V., Eggers, J., 2011. Public transaction costs of agrienvironmental schemes and their determinants-Analysing stakeholders' involvement and perceptions. Ecological Economics 70, 641-650, Elsevier. 
Metz, F., Lieberherr, E., Schmucki, A., Huber, R., 2021. Policy change through negotiated agreements: The case of greening Swiss agricultural policy. Policy Studies Journal 49, 731-756, Wiley-Blackwell.

Meuwissen, M.P.M., Feindt, P.H., Spiegel, A., Termeer, C.J.A.M., Mathijs, E., Mey, Y.d., Finger, R., Balmann, A., Wauters, E., Urquhart, J., Vigani, M., Zawalińska, K., Herrera, H., Nicholas-Davies, P., Hansson, H., Paas, W., Slijper, T., Coopmans, I., Vroege, W., Ciechomska, A., Accatino, F., Kopainsky, B., Poortvliet, P.M., Candel, J.J.L., Maye, D., Severini, S., Senni, S., Soriano, B., Lagerkvist, C.-J., Peneva, M., Gavrilescu, C., Reidsma, P., 2019. A framework to assess the resilience of farming systems. Agricultural Systems 176, 102656, Elsevier.

Meyfroidt, P., 2017. Mapping farm size globally: benchmarking the smallholders debate. Environmental Research Letters 12, 031002, IOP Publishing.

Meyfroidt, P., Börner, J., Garrett, R., Gardner, T., Godar, J., Kis-Katos, K., SoaresFilho, B.S., Wunder, S., 2020. Focus on leakage and spillovers: informing land-use governance in a tele-coupled world. Environmental Research Letters 15, 090202, IOP Publishing.

Meyfroidt, P., Roy Chowdhury, R., de Bremond, A., Ellis, E.C., Erb, K.H., Filatova, T., Garrett, R.D., Grove, J.M., Heinimann, A., Kuemmerle, T., Kull, C.A., Lambin, E.F., Landon, Y., le Polain de Waroux, Y., Messerli, P., Müller, D., Nielsen, J.Ø., Peterson, G.D., Rodriguez García, V., Schlüter, M., Turner, B.L., Verburg, P.H., 2018. Middlerange theories of land system change. Global Environmental Change 53, 52-67, Elsevier.

Mitra, S., Josling, T., 2009. Agricultural export restrictions: Welfare implications and trade disciplines. Position paper agricultural and rural development policy, IPC.

Mittenzwei, K., Britz, W., 2018. Analysing Farm-specific Payments for Norway using the Agrispace Model. Journal of Agricultural Economics 69, 777-793, WileyBlackwell.

Möhring, A., Mack, G., Zimmermann, A., Mann, S., Ferjani, A., 2018. Versorgungssicherheitsbeiträge: Mittel effizienter einsetzen. Agrarforschung Schweiz 9, 348-355, Agroscope.

Möhring, A., Mann, S., 2020. Causes and impacts of the mis-representation of agricultural policy-The case of food supply security payments in Switzerland. Journal of Policy Modeling 42, 466-482, Elsevier.

Möhring, N., Ingold, K., Kudsk, P., Martin-Laurent, F., Niggli, U., Siegrist, M., Studer, B., Walter, A., Finger, R., 2020. Pathways for advancing pesticide policies. Nature Food 1, 535-540, Springer.

Mooney, H., Larigauderie, A., Cesario, M., Elmquist, T., Hoegh-Guldberg, O., Lavorel, S., Mace, G.M., Palmer, M., Scholes, R., Yahara, T., 2009. Biodiversity, climate change, and ecosystem services. Current Opinion in Environmental Sustainability 1, 46-54, Elsevier. 
Moro, D., Sckokai, P., 2013. The impact of decoupled payments on farm choices: Conceptual and methodological challenges. Food Policy 41, 28-38, Elsevier.

Moser, P., 2006. Bäuerliche Familienbetriebe. Historisches Lexikon der Schweiz. https://hls-dhs-dss.ch/de/articles/014176/2006-09-25/.

Mueller, D.C., 2015. Public choice, social choice, and political economy. Public Choice 163, 379-387, Springer.

Muller, A., Ferré, M., Engel, S., Gattinger, A., Holzkämper, A., Huber, R., Müller, M., Six, J., 2017a. Can soil-less crop production be a sustainable option for soil conservation and future agriculture? Land Use Policy 69, 102-105, Elsevier.

Muller, A., Schader, C., El-Hage Scialabba, N., Brüggemann, J., Isensee, A., Erb, K.-H., Smith, P., Klocke, P., Leiber, F., Stolze, M., Niggli, U., 2017b. Strategies for feeding the world more sustainably with organic agriculture. Nature Communications 8 , 1290, Nature Publishing Group.

Musshoff, O., Hirschauer, N., 2010. Modernes Agrarmanagement: Betriebswirtschaftliche Analyse- und Planungsverfahren. Vahlen.

Neary, J.P., 2009. Putting the «New» into New Trade Theory: Paul Krugman's Nobel Memorial Prize in Economics. The Scandinavian Journal of Economics 111, 217-250, Wiley-Blackwell.

Necpalova, M., Lee, J., Skinner, C., Büchi, L., Wittwer, R., Gattinger, A., van der Heijden, M., Mäder, P., Charles, R., Berner, A., Mayer, J., Six, J., 2018. Potentials to mitigate greenhouse gas emissions from Swiss agriculture. Agriculture, Ecosystems \& Environment 265, 84-102, Elsevier.

Nemecek, T., Huguenin-Elie, O., Dubois, D., Gaillard, G., Schaller, B., Chervet, A., 2011. Life cycle assessment of Swiss farming systems: II. Extensive and intensive production. Agricultural Systems 104, 233-245, Elsevier.

Neuenfeldt, S., Gocht, A., Heckelei, T., Ciaian, P., 2018. Explaining farm structural change in the European agriculture: a novel analytical framework. European Review of Agricultural Economics 46, 713-768, Oxford University Press.

Niggli, U. 2021. Alle satt? Ernährung sichern für 10 Milliarden Menschen. Residenz Verlag.

OECD, 2001. Multifunctionality: Towards an analytical framework. OECD Publications.

OECD, 2003. Multifunctionality: The Policy Implications. OECD Publications.

OECD, 2006. Decoupling Agricultural Support from Production. Organization for Economic Cooperation and Development. Policy Brief, November 2006, OECD Publications. 
OECD, 2015. OECD Review of Agricultural Policies: Switzerland 2015. OECD Publications.

OECD, 2020. Agricultural Policy Monitoring and Evaluation 2020. OECD Publication.

Olson, M., 1979. Die Logik des kollektiven Handelns. Kollektivgüter und die Theorie der Gruppen. Ökonomische Theorie der Politik, 287-319, Springer.

Ortiz-Bobea, A., Ault, T.R., Carrillo, C.M., Chambers, R.G., Lobell, D.B.J.a.p.a., 2020. The Historical Impact of Anthropogenic Climate Change on Global Agricultural Productivity. Nature Climate Change 11, 306-312, Nature Publishing Group.

Pe'er, G., Bonn, A., Bruelheide, H., Dieker, P., Eisenhauer, N., Feindt, P.H., Hagedorn, G., Hansjürgens, B., Herzon, I., Lomba, Â., Marquard, E., Moreira, F., Nitsch, H., Oppermann, R., Perino, A., Röder, N., Schleyer, C., Schindler, S., Wolf, C., Zinngrebe, Y., Lakner, S., 2020. Action needed for the EU Common Agricultural Policy to address sustainability challenges. People and Nature 2, 305-316, John Wiley \& Sons.

Pe'er, G., Dicks, L.V., Visconti, P., Arlettaz, R., Báldi, A., Benton, T.G., Collins, S., Dieterich, M., Gregory, R.D., Hartig, F., Henle, K., Hobson, P.R., Kleijn, D., Neumann, R.K., Robijns, T., Schmidt, J., Shwartz, A., Sutherland, W.J., Turbé, A., Wulf, F., Scott, A.V., 2014. EU agricultural reform fails on biodiversity. Science 344, 1090-1092, AAAS.

Pe'er, G., Zinngrebe, Y., Moreira, F., Sirami, C., Schindler, S., Müller, R., Bontzorlos, V., Clough, D., Bezák, P., Bonn, A., Hansjürgens, B., Lomba, A., Möckel, S., Passoni, G., Schleyer, C., Schmidt, J., Lakner, S., 2019. A greener path for the EU Common Agricultural Policy. Science 365, 449-451, AAAS.

Perman, R., Ma, Y., McGilvray, J., Common, M., 2003. Natural resource and environmental economics. Harlow, Pearson Education.

Phalan, B., Onial, M., Balmford, A., Green, R.E., 2011. Reconciling Food Production and Biodiversity Conservation: Land Sharing and Land Sparing Compared. Science 333, 1289-1291, AAAS.

Pinstrup-Andersen, P., 2009. Food security: definition and measurement. Food Security 1, 5-7, Springer.

Piot-Lepetit, I., M'Barek, R., 2011. Methods to Analyse Agricultural Commodity Price Volatility. In: Piot-Lepetit, I., M'Barek, R. (Eds.), Methods to Analyse Agricultural Commodity Price Volatility. 1-11, Springer.

Popp, H.W., 2001. Das Jahrhundert der Agrarrevolution: Schweizer Landwirtschaft und Agrarpolitik im 20. Jahrhundert. Schweizer Agrarmedien.

Poppe, K., Vrolijk, H., 2019. How to measure farm income in the era of complex farms. Paper prepared for presentation at the 171th EAAE Seminar «Measuring and evaluating farm income and well-being of farm families in Europe - Towards a 
shared and broader approach for analysis and policy design?», Tänikon, Switzerland.

Portmann, M., Ritzel, C., 2020. Ex-ante-Evaluation eines Freihandelsabkommens zwischen der Schweiz und den Mercosur-Staaten: Auswirkungen auf die Landwirtschaft., Agroscope Science, 102, 1-25, Agroscope, https://doi.org/10.34776/as102g.

Potter, C., Tilzey, M., 2007. Agricultural multifunctionality, environmental sustainability and the WTO: Resistance or accommodation to the neoliberal project for agriculture? Geoforum 38, 1290-1303, Elsevier.

Power, A.G., 2010. Ecosystem services and agriculture: tradeoffs and synergies. Philosophical Transactions of the Royal Society B: Biological Sciences 365, 29592971, Royal Society Publishing.

Prager, K., 2015. Agri-environmental collaboratives for landscape management in Europe. Current Opinion in Environmental Sustainability 12, 59-66, Elsevier.

Puma, M.J., Bose, S., Chon, S.Y., Cook, B.I., 2015. Assessing the evolving fragility of the global food system. Environmental Research Letters 10, 024007, IOP Publishing.

Python, P., Gresset, F., Réviron, S., 2018. Die Besonderheiten des Schweizer Milchmarktes. Merkblatt Agridea, Agridea.

Rausser, G.C., Goodhue, R.E., 2002. Public policy: Its many analytical dimensions. In: Gardner, B.L., Rausser, G.C. (Eds.), Handbook of Agricultural Economics 2, Chapter 39, 2057-2102, Elsevier.

Rausser, G.C., Swinnen, J., 2011. Governance Structures, Political Economy and Public Policy. American Journal of Agricultural Economics 93, 310-316, WileyBlackwell.

Reisch, L.A., 2021. Shaping healthy and sustainable food systems with behavioural food policy. European Review of Agricultural Economics 48, 665-693, Oxford University Press.

Reviron, S., Python, P., 2018. Analyse der Wertverteilung in der Wertschöpfungskette Milch. Agrarforschung Schweiz 9, 134-141, Agroscope.

Richards, K.R., 2000. Framing Environmental Policy Instrument Choice. Duke Environmental Law \& Policy Forum 10, Duke University School of Law.

Rieder, P., 1983. Grundlagen der Agrarmarktpolitik. Verlag der Fachvereine.

Rieder, P., 1993. Ideen und Geistesgeschichte der europäischen Agrarpolitik. Schriftenreihe/ETH Zürich, Institut für Agrarwirtschaft.

Rieder, P., Anwander Phan-huy, S., 1994. Grundlagen der Agrarmarktpolitik. vdf Hochschulverlag.

Rieder, P., Egger, U., Flückiger, S., 1992. Schweizerische Agrarmärkte. vdf Hochschulverlag. 
Rieder, S., Feige, S., Amberg, H., Hertig, V., 2015. Evaluation der landwirtschaftlichen Absatzförderung. Schlussbericht zuhanden des Bundesamtes für Landwirtschaft (BLW).

Ritzel, C., Mack, G., Portmann, M., Heitkämper, K., El Benni, N., 2020. Empirical evidence on factors influencing farmers' administrative burden: A structural equation modeling approach. PLoS ONE 15, e0241075, Public Library of Science.

Robinson, G.M., 2018. Globalization of Agriculture. Annual Review of Resource Economics 10, 133-160, Annual Reviews.

Rodrik, D., 2018. What Do Trade Agreements Really Do? Journal of Economic Perspectives 32, 73-90, American Economic Association.

Rørstad, P.K., Vatn, A., Kvakkestad, V., 2007. Why do transaction costs of agricultural policies vary? Agricultural Economics 36, 1-11, John Wiley \& Sons.

Sager, F., Hadorn, S., Balthasar, A., \& Mavrot, C. 2021. Politikevaluation. Eine Einführung. Springer Verlag.

Saitone, T.L., Sexton, R.J., 2017. Agri-food supply chain: evolution and performance with conflicting consumer and societal demands. European Review of Agricultural Economics 44, 634-657, Oxford University Press.

Salvatici, L., Nenci, S., 2017. New features, forgotten costs and counterfactual gains of the international trading system. European Review of Agricultural Economics 44, 592-633, Oxford University Press.

Samberg, L.H., Gerber, J.S., Ramankutty, N., Herrero, M., West, P.C., 2016. Subnational distribution of average farm size and smallholder contributions to global food production. Environmental Research Letters 11, 124010, IOP Publishing.

Samuelson, P.A., 1954. The Pure Theory of Public Expenditure. The Review of Economics and Statistics 36, 387-389, MIT Press.

Schader, C., Lampkin, N., Christie, M., Nemecek, T., Gaillard, G., Stolze, M., 2013. Evaluation of cost-effectiveness of organic farming support as an agrienvironmental measure at Swiss agricultural sector level. Land Use Policy 31, 196-208, Elsevier.

Schader, C., Lampkin, N., Muller, A., Stolze, M., 2014. The role of multi-target policy instruments in agri-environmental policy mixes. Journal of Environmental Management 145, 180-190, Elsevier.

Schaub, S., Huber, R., Finger, R., 2020. Tracking societal concerns on pesticides - a Google Trends analysis. Environmental Research Letters 15, 084049, IOP Publishing. Schimmelpfennig, D.E., Pray, C.E., Brennan, M.F., 2004. The impact of seed industry concentration on innovation: a study of US biotech market leaders. Agricultural Economics 30, 157-167, John Wiley \& Sons. 
Schläpfer, F., 2016. Eine Stickstoff-Lenkungsabgabe für die Schweizer Landwirtschaft? Agrarforschung Schweiz 7, 496-503, Agroscope.

Schmid, H.P., Lehmann, B., 2000. Switzerland: agrienvironmental policy outside the European Union. In: Buller, H., Wilson, G.A., Höll, A. (Eds.), Agri-environmental Policy in the European Union. Routledge.

Schmidt, A., Mack, G., Möhring, A., Mann, S., El Benni, N., 2019. Stricter crosscompliance standards in Switzerland: Economic and environmental impacts at farmand sector-level. Agricultural Systems 176, 102664, Elsevier.

Schneider, F., Steiger, D., Ledermann, T., Fry, P., Rist, S., 2012. No-tillage farming: co-creation of innovation through network building. Land Degradation \& Development 23, 242-255, John Wiley \& Sons.

Scott, J., 2017. The future of agricultural trade governance in the World Trade Organization. International Affairs 93, 1167-1184, Oxford University Press.

Sen, A., 2018. Collective choice and social welfare. Harvard University Press.

Senti, R., 2000. WTO. System und Funktionsweise der Welthandelsordnung. Schulthess.

Sexton, R.J., 2013. Market Power, Misconceptions, and Modern Agricultural Markets. American Journal of Agricultural Economics 95, 209-219, Wiley-Blackwell.

Shortle, J.S., Horan, R.D., 2001. The Economics of Nonpoint Pollution Control. Journal of Economic Surveys 15, 255-289, John Wiley \& Sons.

Smith, P., Martino, D., Cai, Z., Gwary, D., Janzen, H., Kumar, P., McCarl, B., Ogle, S., O'Mara, F., Rice, C., Scholes, B., Sirotenko, O., Howden, M., McAllister, T., Pan, G., Romanenkov, V., Schneider, U., Towprayoon, S., Wattenbach, M., Smith, J., 2008. Greenhouse gas mitigation in agriculture. Philosophical Transactions of the Royal Society B: Biological Sciences 363, 789-813, Royal Society Publishing.

Steffen, W., Richardson, K., Rockström, J., Cornell, S.E., Fetzer, I., Bennett, E.M., Biggs, R., Carpenter, S.R., de Vries, W., de Wit, C.A., Folke, C., Gerten, D., Heinke, J., Mace, G.M., Persson, L.M., Ramanathan, V., Reyers, B., Sörlin, S., 2015. Planetary boundaries: Guiding human development on a changing planet. Science 347, 1259855, AAAS.

Steiger, U., Lüthi, S., Schmitt, H.-M., Schüpbach, W., 2016. Evaluation Landschaftsqualitätsbeiträge. Schlussbericht zuhanden des Bundesamts für Landwirtschaft.

Sterner, T., Barbier, E.B., Bateman, I., van den Bijgaart, I., Crépin, A.-S., Edenhofer, O., Fischer, C., Habla, W., Hassler, J., Johansson-Stenman, O., Lange, A., Polasky, S., Rockström, J., Smith, H.G., Steffen, W., Wagner, G., Wilen, J.E., Alpízar, F., Azar, C., Carless, D., Chávez, C., Coria, J., Engström, G., Jagers, S.C., Köhlin, G., Löfgren, Å., Pleijel, H., Robinson, A., 2019. Policy design for the Anthropocene. Nature Sustainability 2, 14-21, Nature Pulishing Group. 
Stevanović, M., Popp, A., Lotze-Campen, H., Dietrich, J.P., Müller, C., Bonsch, M., Schmitz, C., Bodirsky, B.L., Humpenöder, F., Weindl, I., 2016. The impact of high-end climate change on agricultural welfare. Science 2, e1501452, AAAS.

Stiglitz, J.E., 2007. Making globalization work. WW Norton.

Stolze, M., Frick, R., Schmid, O., Stöckli, S., Chevillat, V., Dubbert, M., Fleury, P., Neuner, H., Plaikner, M., Nitsch, H., 2015. Ergebnisorientierte Massnahmen zur Förderung der Biodiversität in der Berglandwirtschaft-Ein Handbuch für die Politik. Forschungsinstitut für biologischen Landbau (FiBL).

Swinnen, J.F., 2018. The political economy of agricultural and food policies. Springer.

Swinnen, J.F.M., 2010. The Political Economy of Agricultural and Food Policies: Recent Contributions, New Insights, and Areas for Further Research. Applied Economic Perspectives and Policy 32, 33-58, Oxford University Press.

Tauger, M.B., 2020. Agriculture in world history. Routledge.

Tilman, D., Balzer, C., Hill, J., Befort, B.L., 2011. Global food demand and the sustainable intensification of agriculture. Proceedings of the National Academy of Sciences 108, 20260-20264, National Academy of Science.

Tilman, D., Cassman, K.G., Matson, P.A., Naylor, R., Polasky, S., 2002. Agricultural sustainability and intensive production practices. Nature 418, 671-677.

Tilman, D., Clark, M., 2014. Global diets link environmental sustainability and human health. Nature 515, 518-522, Macmillan Publishers.

Timmer, C.P., 2009. A world without agriculture: the structural transformation in historical perspective. AEI Press.

Tschumi, M., Albrecht, M., Bärtschi, C., Collatz, J., Entling, M.H., Jacot, K., 2016. Perennial, species-rich wildflower strips enhance pest control and crop yield. Agriculture, Ecosystems \& Environment 220, 97-103, Elsevier.

Tschumi, M., Albrecht, M., Entling, M.H., Jacot, K., 2015. High effectiveness of tailored flower strips in reducing pests and crop plant damage. Proceedings of the Royal Society B: Biological Sciences 282, 20151369, Royal Society Publishing.

Ulber, L., Klimek, S., Steinmann, H.-H., Isselstein, J., Groth, M., 2011. Implementing and evaluating the effectiveness of a payment scheme for environmental services from agricultural land. Environmental Conservation 38, 464-472, Cambridge University Press.

UN, 2016. United Nations Department of Economic Social Affairs: The Sustainable Development Goals Report 2016. United Nations.

UN, 2020. United Nations: The Sustainable Development Goals Report. United Nations. 
Van Huylenbroeck, G., Vandermeulen, V., Mettepenningen, E., Verspecht, A., 2007. Multifunctionality of Agriculture: A Review of Definitions, Evidence and Instruments. Living Rev. Landscape Res. 1, (2007), URL (cited on 28.10.2009): http://www.livingreviews.org//rIr-2007-3Leibniz.

van Tongeren, F., 2008. Agricultural Policy Design and Implementation. OECD Publishing.

Vandecandelaere, E., Teyssier, C., Barjolle, D., Jeanneaux, P., Fournier, S., Beucherie, O., 2018. Strengthening sustainable food systems through geographical indications: an analysis of economic impacts. FAO - Food and Agriculture Organisation.

Varian, H.R., 2016. Grundzüge der Mikroökonomik. De Gruyter.

Vatn, A., 2002. Multifunctional agriculture: some consequences for international trade regimes. European Review of Agricultural Economics 29, 309-327, Oxford University Press.

Vogeler, C.S., 2017. Farm Animal Welfare Policy in Comparative Perspective: Determinants of Cross-national Differences in Austria, Germany, and Switzerland. European Policy Analysis 3, 20-47, John Wiley \& Sons.

Von Braun, J., 2015. Welternährung und Nachhaltigkeit. oekom Verlag.

Vroege, W., Dalhaus, T., Finger, R., 2019. Index insurances for grasslands - A review for Europe and North-America. Agricultural Systems 168, 101-111, Elsevier.

Walter, A., Finger, R., Huber, R., Buchmann, N., 2017. Opinion: Smart farming is key to developing sustainable agriculture. Proceedings of the National Academy of Sciences 114, 6148-6150, National Academy of Science.

Walton, A., 2010. What is Fair Trade? Third World Quarterly 31, 431-447, Talyor \& Francis.

Watson, D.D., 2017. The political economy of food price policy during the global food price crisis of 2006-2008. Food Security 9, 497-509, Springer.

Webber, H., Lischeid, G., Sommer, M., Finger, R., Nendel, C., Gaiser, T., Ewert, F., 2020. No perfect storm for crop yield failure in Germany. Environmental Research Letters 15, 104012, IOP Publishing.

Weiss, J., 2017. Die Schweizer Landwirtschaft stirbt leise. Lasst die Bauern wieder Bauern sein. Orell Füssli.

Wey, C., Gösser, N., 2019. Eine Bewertung der Rolle des Grenzschutzes auf die landwirtschaftlichen Betriebe in der Schweiz und ihre vorgelagerten Industrien. Eine Studie im Auftrag des Bundesamts für Landwirtschaft.

Widmer, T., 2017. Die Zukunft des landwirtschaftlichen Grenzschutzes. Institut für Politikwissenschaft, Universität Zürich. 
Williams, J.C., 1982. The Origin of Futures Markets. Agricultural History 56, 306316, Agricultural History Society.

Wilson, G. A., 2007. Multifunctional agriculture a transition theory perspective. Wallingford: CABI.

Wohlmeyer, H., Quendler, T., 2017. The WTO, agriculture and sustainable development. Routledge.

WTO, 2020a. Groups in the agriculture negotiations. In: World Trade Organization (https://www.wto.org/english/tratop_e/agric_e/negoti_groups_e.htm). Abgerufen November 2021.

WTO, 2020b. What is the WTO? In: World Trade Organization (https://www.wto.org/english/thewto_e/whatis_e/whatis_e.htm). Abgerufen November 2021

WTO, 2020c. The WTO must not continue as it is. In: World Trade Organization (https://www.wto.org/english/news_e/news20_e/ddgaw_10dec20_e.htm). Abgerufen November 2021

Wunder, S., Brouwer, R., Engel, S., Ezzine-de-Blas, D., Muradian, R., Pascual, U., Pinto, R., 2018. From principles to practice in paying for nature's services. Nature Sustainability 1, 145-150, Nature Publishing Group.

Wünscher, T., Engel, S., Wunder, S., 2008. Spatial targeting of payments for environmental services: A tool for boosting conservation benefits. Ecological Economics 65, 822-833, Elsevier.

Wüpper, D., Huber, R. 2021. A Comparison of Action- and Results-Based AgriEnvironmental Payments in Switzerland. Journal of Agricultural Economics.

ZDA, 2020. Zentrum für Demokratie Aarau: Politische Bildung.

Zellweger-Fischer, J., Kéry, M., Pasinelli, G., 2011. Population trends of brown hares in Switzerland: The role of land-use and ecological compensation areas. Biological Conservation 144, 1364-1373, Elsevier.

Zimmermann, A., Baumgartner, D., Nemecek, T., Gaillard, G., 2011. Are public payments for organic farming cost-effective? Combining a decision-support model with LCA. The International Journal of Life Cycle Assessment 16, 548-560, Springer.

Zingg, S., Grenz, J., Humbert, J.-Y., 2018. Landscape-scale effects of land use intensity on birds and butterflies. Agriculture, Ecosystems \& Environment 267, 119128, Elsevier.

Zingg, S., Ritschard, E., Arlettaz, R., Humbert, J.-Y., 2019. Increasing the proportion and quality of land under agri-environment schemes promotes birds and butterflies at the landscape scale. Biological Conservation 231, 39-48, Elsevier. 


\section{Einführung in die Schweizer Agrarpolitik}

Dieses Buch vermittelt Basiswissen der Schweizer Agrarpolitik und ordnet dieses aus einer agrarökonomischen Sicht ein. Das Buch beschreibt die theoretischen und konzeptionellen Grundlagen der Agrarpolitik und erörtert die Wirkungsweise von agrarpolitischen Instrumenten. Diese Grundlagen werden mit Blick auf internationale Entwicklungen, die Geschichte der Agrarpolitik und die Schweizer Agrargesetzgebung vertieft. Zudem werden die Prozesse und Institutionen der Schweizer Agrarpolitik aus konzeptioneller Sicht beschrieben und illustriert. Das Buch wirft zuletzt auch einen Blick auf die zukünftigen

\section{DAECP}

\section{V/llf}

www.vdf.ethz.ch verlag@vdf.ethz.ch
Herausforderungen der Agrarpolitik: die Digitalisierung der Landwirtschaft, den Klimawandel und die Transformation der bestehenden Agrarpolitik in eine ganzheitliche Agrar- und Ernährungspolitik. Die Agrarpolitik als integrale Ernährungspolitik zu konzipieren, würde es ermöglichen, die ökonomischen, ökologischen und sozialen Herausforderungen der Landwirtschaft ganzheitlich anzugehen. 